\title{
EXPERIMENTELLE BESTIMMUNG DER DEPOSITIONSGESCHWINDIGKEIT LUFTGETRAGENER PARTIKEL MIT HILFE DER EDDY - KOVARIANZMETHODE ÜBER EINEM FICHTENALTBESTAND IM SOLLING
}

\author{
Dissertation \\ zur Erlangung des Doktorgrades \\ der Mathematisch-Naturwissenschaftlichen Fakultäten \\ der Georg-August-Universität zu Göttingen
}

vorgelegt von

Matthias Richard Bleyl

aus Berlin

Göttingen 2001 
D7

Referent: Prof. Dr. G. Gerold

Korreferent: Prof. Dr. G. Gravenhorst

Tag der mündlichen Prüfung: 30.01.2002 
"Progress in the study of vegetation and the atmosphere, as in the whole of ecology, depends on maintaining a sensible balance between all these essential activities: the development of instruments and recording systems, the interpretation of measurements, the construction of mathematical models, and most of all on the collaboration of micrometeorologists and ecologists who are prepared to learn from each other"

MONTEITH (1975) 


\section{Inhaltsverzeichnis}

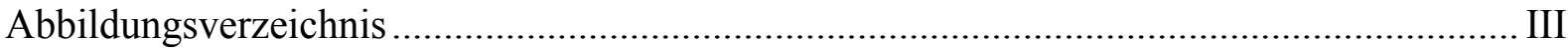

Verzeichnis der verwendeten Symbole …….............................................................. V

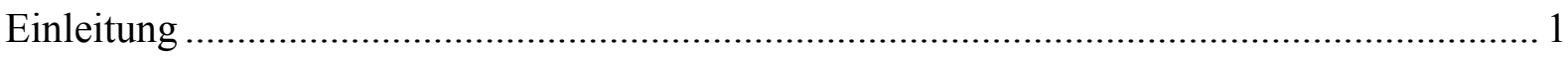

1 Theoretische Überlegungen zum Stoffaustausch zwischen Atmosphäre und Vegetation .. 3

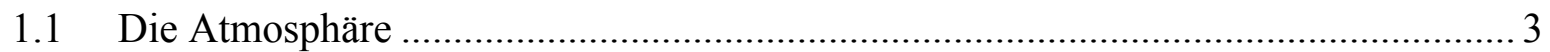

1.1.1 Detailbetrachtung Atmosphäre: Atmosphärische Partikel .................................... 4

1.1.1.1 Physikalische und chemische Charakterisierung luftgetragener Partikel ........5

1.1.1.2 Der Lebenslauf luftgetragener Partikel ............................................................. 7

1.1.1.3 Auswirkungen des atmosphärischen Aerosols auf das Klima ........................ 8

1.1.1.4 Messtechnische Erfassung von Partikeln .................................................... 9

1.1.2 Detailbetrachtung Atmosphäre: Die Planetarische Grenzschicht ....................... 10

1.2 Die vegetationsbestandene Erdoberfläche aus meteorologischer Sicht ..................... 14

1.2.1 Allgemeine Charakterisierung der vegetationsbestandenen Oberfläche................ 14

1.2.2 Quantitative Beschreibung der vegetationsbestandenen Oberfläche aus mikrometeorologischer Sicht ...................................................................... 15

1.3 Stoff- und Energieaustausch zwischen Atmosphäre und Erdoberfläche ................... 15

1.3.1 Detailbetrachtung Stoff- und Energieaustausch: Der Energieumsatz an der

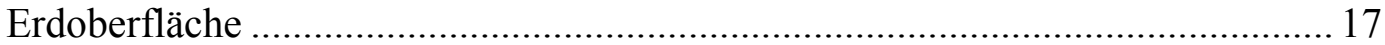

1.3.2 Detailbetrachtung Stoff- und Energieaustausch: Spurenstoffdeposition............... 18

1.3.2.1 Mechanismen der Trockenen Deposition luftgetragener Partikel................... 20

1.3.2.2 Quantifizierung der Trockenen Deposition luftgetragener Partikel............... 22

1.3.2.3 Methoden der experimentellen Bestimmung der Trockenen Deposition luftgetragener Partikel ................................................................................ 23

1.3.2.4 Trockene Deposition und Depositionsgeschwindigkeit luftgetragener Partikel

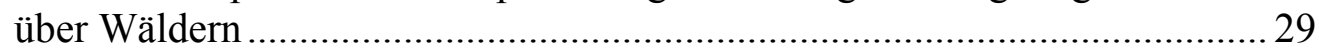

2 Mikrometeorologische Methoden zur Bestimmung vertikaler Partikelflussdichten unter besonderer Berücksichtigung der Eddy - Kovarianzmethode....................................... 31

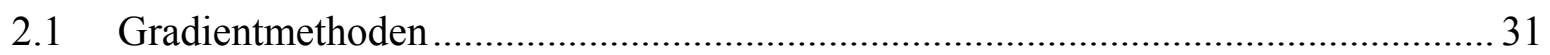

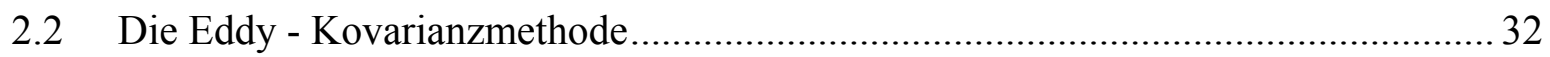

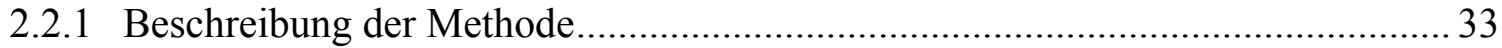

2.2.2 Voraussetzungen für die Durchführung der Eddy - Kovarianzmethode ................ 35

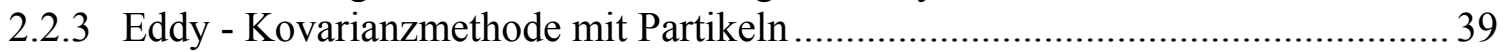

2.2.4 Korrekturen für die Eddy - Kovarianzmethode................................................ 40

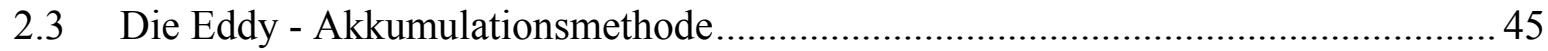


2.4 Bestimmung vertikaler Flussdichten atmosphärischer Partikel im Zuge der

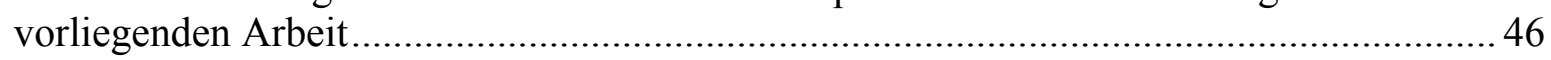

3 Eigene Messungen zur Trockenen Deposition von luftgetragenen Partikeln .................. 48

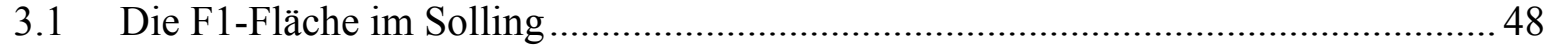

3.1.1 Lage und Ausstattung der F1-Fläche.............................................................. 48

3.1.2 Bisherige Eddy - Kovarianzmessungen und Untersuchungen zum Partikeleintrag

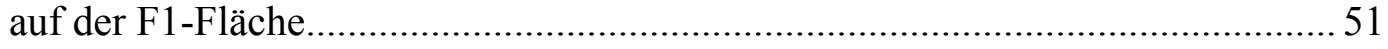

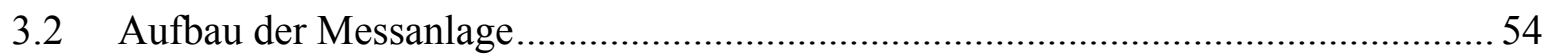

3.2.1 Zentrale Komponenten: PCS-2000 und USAT 3/1 ........................................ 54

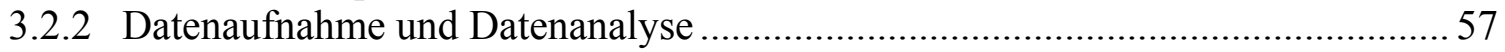

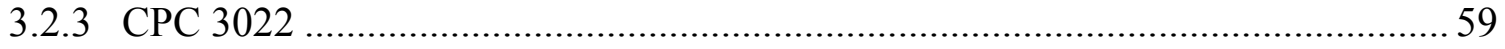

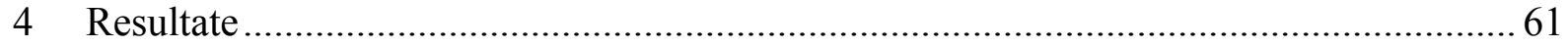

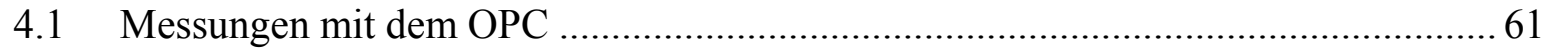

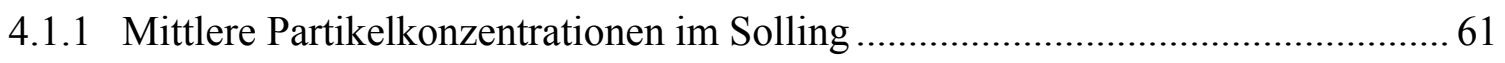

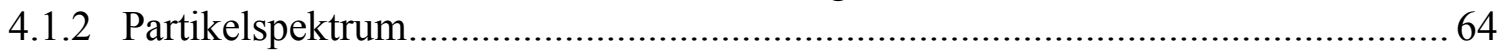

4.1.3 Zeitlich hochaufgelöstes Signal der Partikelkonzentration .................................... 71

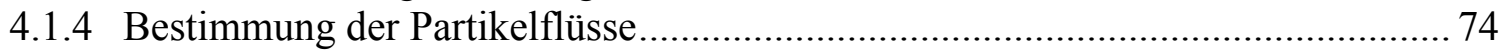

4.1.5 Einfluss der relativen Luftfeuchte auf die Bestimmung der Partikelflüsse........... 82

4.1.6 Verbesserung der Datenqualität: Modifikation des Datensatzes.......................... 86

4.1.7 Mittlere Beträge und Abhängigkeiten von meteorologischen Größen.................. 89

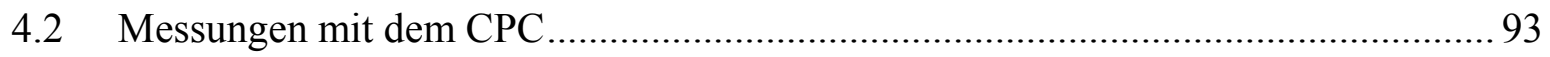

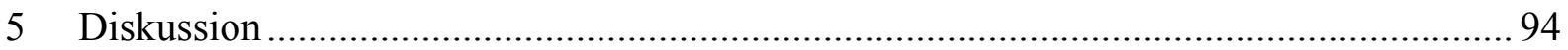

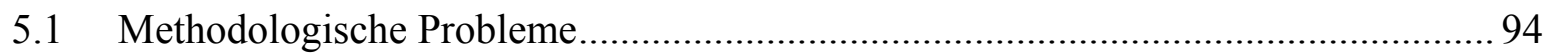

5.1.1 Der PCS-2000 als meteorologisches Messgerät: Probleme und potentielle

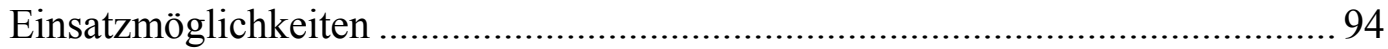

5.1.2 Notwendige Randbedingungen für die Quantifizierung der Partikeldeposition mit Hilfe der Eddy - Kovarianzmethode ............................................................... 95

5.2 Partikelkonzentration und Partikelspektrum ........................................................... 99

5.3 Depositionsgeschwindigkeit luftgetragener Partikel über Wald............................ 100

5.3.1 Vergleich der gefundenen Resultate mit Literaturangaben ................................. 100

5.3.2 Abhängigkeit der Depositionsgeschwindigkeit von der atmosphärischen

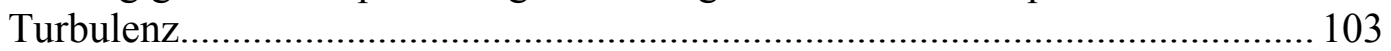

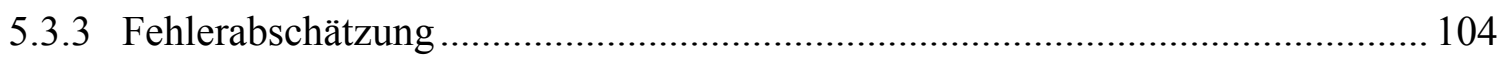

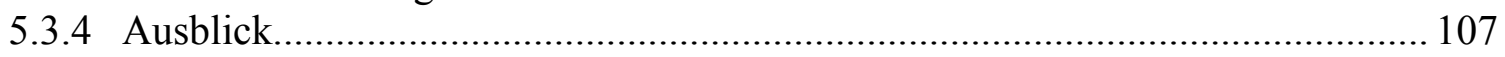

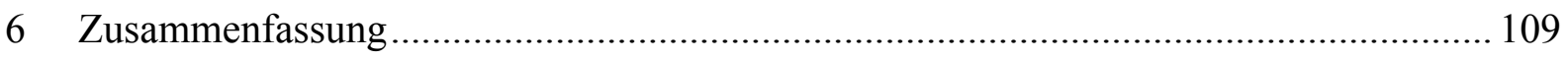

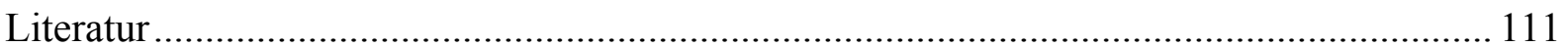

Anhang: Untersuchungen von Sonics der Firma Metek im Windkanal der ETH Zürich im Rahmen der vorliegenden Arbeit 
Abbildungsverzeichnis

\section{Abbildungsverzeichnis}

Abb. 1: Vertikale Gliederung der Atmosphäre: meteorologische, mikrometeorologische und landschaftsökologische Betrachtung im Vergleich

Abb. 2: Fichtennadeln unter dem Elektronenmikroskop. Ausschnitte einer mit Ultraschall gereinigten Nadeloberfläche.

Abb. 3: Fichtennadeln unter dem Elektronenmikroskop: Ausschnitte der Oberfläche einer unter Reinluft ausgetriebenen Nadel mit stäbchenförmiger Wachsstruktur .... 26

Abb. 4: Berechnete Halbstunden-Werte des fühlbaren Wärmeflusses über Wald aus 1 $\mathrm{Hz}, 0,1 \mathrm{~Hz}$ und 0,01 Hz Daten als Funktion der Resultate aus $10 \mathrm{~Hz}-$ Daten ............ 38

Abb. 5: Ausgewählte Effekte der Korrektur der Messwerte des Ultraschallanemometers..... 41

Abb. 6: Geographische Lage der Solling-Untersuchungsflächen ......................................... 48

Abb. 7: Klima des Sollings: Windrose für das Jahr 2000 auf der F1-Fläche ......................... 49

Abb. 8: Blick über die 116jährigen Fichten der F1-Fläche in Richtung Nord sowie

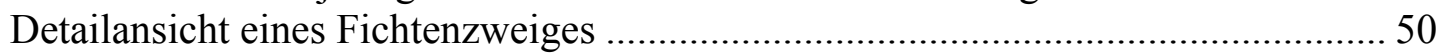

Abb. 9: Ergebnisse der bisherigen Depositionsmessungen auf der F1-Fläche ....................... 53

Abb. 10:Aerosolspektrometer PCS-2000 der Firma Palas und schematische Darstellung des Messgerätes.................................................................................................... 55

Abb. 11:Ansaugvorrichtung und Ultraschallanemometer USAT 3/1 in $39 \mathrm{~m}$ auf der F1-

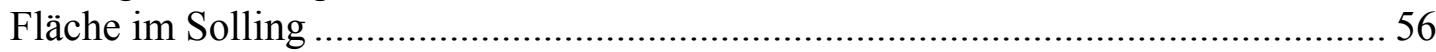

Abb. 12: Schema der Messanlage mit Sonic, Ansaugeinrichtung, OPC sowie

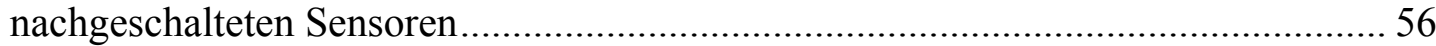

Abb. 13:Steuerung des Eddy-Programms vom einem beliebigen, an das Internet angeschlossenen Rechner............................................................................... 57

Abb. 14:Mit dem CPC TSI 3022 in Kombination mit einem Größen-Fraktionierer

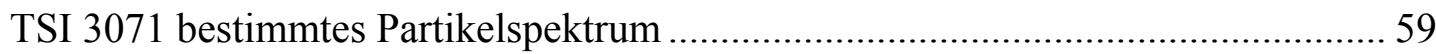

Abb. 15:Mittlere Tagesgänge der Konzentration der fünf kleinsten Partikelklassen sowie der relativen Feuchte

Abb. 16:Partikelkonzentration, relative Luftfeuchte und Globalstrahlung für den 5. bis 8. Mai 2000

Abb. 17:Tägliche Rückwärtstrajektorien für den Solling.

Abb. 18:Verschiebung des Konzentrationsmaximums im Partikelspektrum zu Gunsten größerer Partikel als Folge des Anstiegs der relativen Feuchte ................................ 65

Abb. 19:Webcam - Bilder und Partikelspektrum für den 24. März 2001 ...................................66

Abb. 20:Webcam - Bilder und Partikelspektrum für den 30. März 2001 .................................66

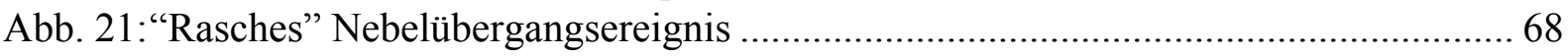

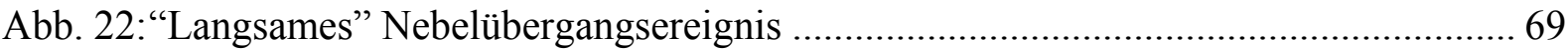

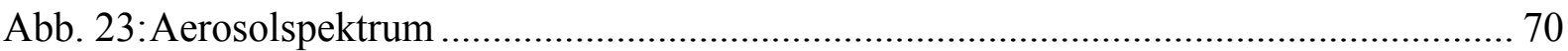

Abb. 24:Gemittelte Powerspektren für die Konzentration der fünf kleinsten

Partikelklassen sowie für horizontale Windgeschwindigkeit und Lufttemperatur .... 71

Abb. 25:Zufällige Verteilung der Partikel im Raum und beliebig gewählter Ausschnitt zur Bestimmung der Partikeldichte 
Abbildungsverzeichnis

Abb. 26:Berechnete Tagesgänge der Depositionsgeschwindigkeit luftgetragener Partikel für die sechs kleinsten Größenklassen des OPC für den 23./24.8.00....

Abb. 27:Mittlere Tagesgänge der Depositionsgeschwindigkeit für die 8 kleinsten

Klassen des OPC 76

Abb. 28:Vorausgesagte Standardfehler für die Depositionsgeschwindigkeit..... 78

Abb. 29:Vergleich des vorausgesagten mit dem real beobachteten Standardfehler der Depositionsgeschwindigkeit für drei ausgewählte Größenklassen 78

Abb. 30:Berechnete Tagesgänge der Depositionsgeschwindigkeit luftgetragener Partikel für die sechs kleinsten Größenklassen des OPC für den 23./24.8.00 sowie nach Gleichung (37) vorausgesagter Standardfehler der Messwerte

Abb. 31:Vergleich der gemessenen Depositionsgeschwindigkeit (absolut) mit dem Standardfehler für fünf ausgewählte Größenklassen des OPC

Abb. 32:Vorausgesagte Standardfehler für die Depositionsgeschwindigkeit........................ 82

Abb. 33:Depositionsgeschwindigkeiten für den 25.11.00 …............................................... 83

Abb. 34:Berechnete Depositionsgeschwindigkeiten für die sechs kleinsten

Partikelklassen als Funktion der relativen Feuchte der Umgebungsluft.

Abb. 35:Partikelkonzentration für vier ausgewählte Größenklassen bei einer relativen

Luftfeuchte $>98 \%$ als Funktion der Zeit.

Abb. 36:Prozentualer Anteil auszuselektierender Messwerte

Abb. 37:Mittlere Tagesgänge der Depositionsgeschwindigkeit für die 8 kleinsten

Klassen des OPC

Abb. 38:Depositionsgeschwindigkeit (alle Klassen) als Funktion von $u *$ für labile und stabile Schichtung

Abb. 39:Depositionsgeschwindigkeit der drei kleinsten Partikelklassen als Funktion von

$u *$ für neutrale Schichtung.

Abb. 40:Depositionsgeschwindigkeit der drei kleinsten Partikelklassen als Funktion von $u *$ für labile und stabile Schichtung 92

Abb. 41:Vergleich der Ergebnisse der bisherigen Depositionsmessungen auf der F1-

Fläche mit den Ergebnisses der vorliegenden Arbeit

Abb. 42:Vergleich der berechneten Depositionsgeschwindigkeit mit modellierten

Werten von CONSTANTIN (1993) 102

Abb. 43:Seiten- und Frontalansicht des großen Windkanals an der ETH Zürich.................. 125

Abb. 44:Kopfüber montierter USA1 Sensorkopf in der Messstrecke des Windkanals ......... 126

Abb. 45:Abweichung der gemessenen Windgeschwindigkeit für das Sonic SN 99.03006 .. 130

Abb. 46:Abweichung des Betrages der Vertikalkomponente für das Sonic SN 99.03006... 131

Abb. 47:Abweichung der horizontalen Windrichtung für das Sonic SN 99.03006.............. 132

Abb. 48:Abweichung der gemessenen Windgeschwindigkeit für das Sonic SN 94.1229 .... 133

Abb. 49:Abweichung des Betrages der Vertikalkomponente für das Sonic SN 94.1229..... 134

Abb. 50:Abweichung der horizontalen Windrichtung für das Sonic SN 94.1229............... 135

Abb. 51:Abweichung der gemessenen Windgeschwindigkeit für das Sonic SN 98.12015 .. 136

Abb. 52:Abweichung des Betrages der Vertikalkomponente für das Sonic SN 98.12015 ... 137

Abb. 53:Abweichung der horizontalen Windrichtung für das Sonic SN 98.12015.............. 138 


\section{Verzeichnis der verwendeten Symbole}

\begin{tabular}{|c|c|c|}
\hline$B N$ & Flussdichte (Transportrate) im Bestandsniederschlag & $\mathrm{kg} /\left(\mathrm{m}^{2} \mathrm{~s}\right)$ \\
\hline$c$ & $\begin{array}{l}\text { Luftkonzentration (Dichte) einer Eigenschaft eig; bei Partikeln: } \\
\text { Partikelanzahldichte }\end{array}$ & $\begin{array}{l}{[\mathrm{eig}] / \mathrm{cm}^{3} \text { bzw. }} \\
1 / \mathrm{cm}^{3}\end{array}$ \\
\hline$c_{p}$ & spezifische Wärmekapazität von Luft bei konstantem Druck & $\mathrm{J} /(\mathrm{K} \mathrm{kg})$ \\
\hline$d$ & Partikeldurchmesser; Verdrängungsschichtdicke & $\mu \mathrm{m} b z w . \mathrm{m}$ \\
\hline$D$ & molekularer Diffusionskoeffizient & $\mathrm{m}^{2} / \mathrm{s}$ \\
\hline$E_{v d}$ & Standardfehler der berechneten Depositionsgeschwindigkeit & $\mathrm{cm} / \mathrm{s}$ \\
\hline$F$ & $\begin{array}{l}\text { Flussdichte (Transportrate) einer Eigenschaft eig; bei Partikeln: } \\
\text { vertikaler Partikelfluss }\end{array}$ & $\begin{array}{c}\left.\text { [eig] / ( } \mathrm{m}^{2} \mathrm{~s}\right) b z w \\
1 /\left(\mathrm{m}^{2} \mathrm{~s}\right)\end{array}$ \\
\hline$F N$ & $\begin{array}{l}\text { Flussdichte (Transportrate) eines Spurenstoffs im } \\
\text { Freilandniederschlag }\end{array}$ & $\mathrm{kg} /\left(\mathrm{m}^{2} \mathrm{~s}\right)$ \\
\hline$g$ & Gravitationsbeschleunigung (Normwert 9.81) & $\mathrm{m} / \mathrm{s}$ \\
\hline$G$ & Bodenwärmestrom & $\mathrm{W} / \mathrm{m}^{2}$ \\
\hline$H$ & fühlbarer Wärmestrom & $\mathrm{W} / \mathrm{m}^{2}$ \\
\hline$K$ & Konstante (allgemein) & - \\
\hline$k_{a}$ & Karman-Konstante $(\approx 0.4)$ & - \\
\hline$K_{e}$ & turbulenter Diffusionskoeffizient der Eigenschaft eig & $\mathrm{m}^{2} / \mathrm{s}$ \\
\hline$L$ & Monin-Obuchov-Mischungslänge & $\mathrm{m}$ \\
\hline$L A I$ & allseitiger Blattflächenindex & - \\
\hline$L E$ & latenter Wärmestrom & $\mathrm{W} / \mathrm{m}^{2}$ \\
\hline$M M D$ & Mittlerer Massendurchmesser & $\mu \mathrm{m}$ \\
\hline$p$ & Luftdruck & $\mathrm{N} / \mathrm{m}^{2}$ \\
\hline$P$ & Anzahl gezählter Teilchen pro Zeiteinheit & - \\
\hline$q$ & spezifische Feuchte der Luft & $\mathrm{g} / \mathrm{kg}$ \\
\hline$r$ & Partikelradius; Transportwiderstand & $\mu \mathrm{m} b z w . \mathrm{s} / \mathrm{cm}$ \\
\hline$r_{a}$ & $\begin{array}{l}\text { aerodynamischer Widerstand zwischen Messhöhe und } \\
\text { Erdoberfläche }\end{array}$ & $\mathrm{s} / \mathrm{cm}$ \\
\hline
\end{tabular}


Verzeichnis der verwendeten Symbole

\begin{tabular}{|c|c|c|}
\hline $\operatorname{Re}$ & Reynoldszahl & - \\
\hline$R_{n}$ & Strahlungsbilanz & $\mathrm{W} / \mathrm{m}^{2}$ \\
\hline$t$ & Zeit & $\mathrm{s}$ \\
\hline$T$ & Lufttemperatur (absolut) & $\mathrm{K}$ \\
\hline $\begin{array}{l}u, v \\
w\end{array}$ & $\begin{array}{l}\text { longitudinale, laterale und vertikale Komponente des } \\
\text { Windvektors }\end{array}$ & $\mathrm{m} / \mathrm{s}$ \\
\hline$u *$ & Schubspannungsgeschwindigkeit & $\mathrm{m} / \mathrm{s}$ \\
\hline$U$ & Betrag der horizontalen Windgeschwindigkeit & $\mathrm{m} / \mathrm{s}$ \\
\hline$v_{d}$ & Depositionsgeschwindigkeit luftgetragener Partikel & $\mathrm{m} / \mathrm{s}$ \\
\hline$v_{d s}$ & Depositionsgeschwindigkeit an der Erdoberfläche & $\mathrm{m} / \mathrm{s}$ \\
\hline$v_{g}$ & Sedimentationsgeschwindigkeit & $\mathrm{m} / \mathrm{s}$ \\
\hline$V$ & Volumen & $\mathrm{cm}^{3}$ \\
\hline$z$ & vertikale Raumkoordinate (Höhe über Erdboden) & $\mathrm{m}$ \\
\hline$z_{0}$ & Rauhigkeitslänge & $\mathrm{m}$ \\
\hline$\beta$ & $\begin{array}{l}\text { Bowen Verhältnis (Verhältnis zwischen fühlbarem und latentem } \\
\text { Wärmestrom); Proportionskonstante }\end{array}$ & - \\
\hline$\phi$ & skalare Eigenschaft der Atmosphäre & - \\
\hline$\eta$ & Viskosität von Luft $\left(1,8310^{-5}\right.$ bei $20^{\circ} \mathrm{C}$ und Normaldruck) & $\mathrm{kg} /(\mathrm{m} \mathrm{s})$ \\
\hline$\lambda_{w}$ & Spezifische Verdampfungswärme von Wasser & $\mathrm{J} / \mathrm{kg}$ \\
\hline$\mu$ & $\begin{array}{l}\text { Verhältnis der Molekulargewichte von Wasserdampf und } \\
\text { trockener Luft }\left(0.622^{-1}\right)\end{array}$ & - \\
\hline$\rho$ & Luftdichte & $\mathrm{kg} / \mathrm{m}^{3}$ \\
\hline$\sigma$ & Standardabweichung einer Eigenschaft eig & [eig] \\
\hline$\tau$ & Schubspannung & $\mathrm{N} / \mathrm{m}^{2}$ \\
\hline$\tau$ & Mittelungszeitraum & $\mathrm{s}$ \\
\hline $\mathrm{u}$ & $\begin{array}{l}\text { kinematische Zähigkeit von Luft }\left(1.510^{-5} \text { bei } 0^{\circ} \mathrm{C} \text { und }\right. \\
\text { Normaldruck) }\end{array}$ & $\mathrm{m}^{2} / \mathrm{s}$ \\
\hline
\end{tabular}

Wie in der Mikrometeorologie üblich, soll mit $\bar{x}$ das zeitliche Mittel einer Größe $x$ und mit $x^{\prime}$ die Abweichung des Augenblickwerts vom Mittelwert (also: $\bar{x}-x$ ) bezeichnet werden. 


\section{Einleitung}

Die Meteorologie befasst sich mit der Beschreibung, Erklärung und Simulation der Vorgänge und Erscheinungen in der Atmosphäre. Sie hat sich im Laufe der letzten 100 Jahre von der "Lehre von den Witterungserscheinungen" als "Prototyp jener Wissenschaften, die es trotz ernstlicher Bemühungen noch zu keinen oder fast keinen Gesetzen gebracht haben" (EXNER, 1925) zu einer "modernen Meteorologie" entwickelt, die als "Teilgebiet der exakten Naturwissenschaften" (FORTAK, 1982) die gesamte Physik und Chemie der Atmosphäre und auch die der Atmosphären anderer Planeten umfasst.

Die Vorgänge und Erscheinungen in der Atmosphäre stehen in komplexen Wechselwirkungen zu Vorgängen in anderen Teilen des dynamischen Systems Erde. Die Atmosphäre ist in die vielfältig vernetzten Stoff- und Wirkungskreisläufe einbezogen, in denen ein Grossteil der in und auf der Erde ablaufenden Prozesse organisiert ist. Indem die Meteorologie diesem Umstand Rechnung trägt und sich nicht nur auf die Beschreibung des Systemkomplexes Atmosphäre selbst einschließlich aller darin auftretenden internen Wechselwirkungen beschränkt, sondern auch die externen Wechselwirkungen mit anderen Teilsystemen der Erde, wie der Pedosphäre, der Hydrosphäre und der Biosphäre, zu ihrem Gegenstand macht, kann sie heute als Teil einer umfassenden "Umweltwissenschaft" begriffen werden (WARNECKE, 1997).

Durch den kontinuierlichen und starken Ausstoß von Stoffen infolge menschlicher Tätigkeit (Industrie, Gewerbe, Landwirtschaft, Verkehr, Haushalte) sind in der Atmosphäre und dadurch mittelbar in terrestrischen und aquatischen Ökosystemen Veränderungen initiiert worden, deren mittel- und langfristigen Folgen derzeit nicht abzusehen sind. Um diese zukünftigen Entwicklungen vorhersagen und beeinflussen zu können, werden zahlreiche Anstrengungen unternommen, die qualitative und quantitative Entwicklung sowohl der Atmosphäre als auch der betroffenen Ökosysteme zu modellieren. Eine solche Modellierung setzt notwendiger Weise detaillierte Kenntnisse zum Stoff- und Energieaustausch zwischen Atmosphäre und der vegetationsbestandenen Oberfläche voraus. Diese Kenntnisse können jedoch nur mit Hilfe von Feldmessungen an und in den betroffenen Ökosystemen gewonnen werden.

Die vorliegende Arbeit beschäftigt sich mit einem Aspekt des Stoffaustausches zwischen der Atmosphäre und der vegetationsbestandenen Oberfläche: der Quantifizierung der Trockenen Deposition luftgetragener Partikel ${ }^{1}$ in einem Fichtenforst. Für Wälder und Forste ist der Stoffeintrag aus der Atmosphäre von ganz besonderer Bedeutung, da diese Ökosysteme im Gegensatz zu landwirtschaftlichen Nutzflächen in der Regel nicht gedüngt werden, und der atmosphärische Stoffeintrag somit faktisch die einzige Quelle von Schad- und Nährstoffen für das System ist. Ziel dieser Arbeit war es, die Depositionsgeschwindigkeit luftgetragener

\footnotetext{
${ }^{1}$ Partikel (lat. particula): Teilchen
} 
Partikel exemplarisch für einen Fichtenaltbestand im Solling zeitlich hoch aufgelöst und als Funktion der Partikelgröße sowie der atmosphärischen Randbedingungen zu bestimmen. Als Untersuchungsmethode wurde die Eddy-Kovarianzmethode gewählt. Da für den untersuchten Bestand bereits Messungen der Trockenen Deposition mit anderen Methoden durchgeführt wurden, liegt ein besonderer Schwerpunkt dieser Arbeit auch auf der Frage, ob, und unter welchen Bedingungen, die gewählte Methode für die Bestimmung der Partikeldeposition anwendbar ist.

Im ersten Kapitel der Arbeit werden zunächst einige allgemeine Grundlagen zum Stoffaustausch zwischen der Atmosphäre und der vegetationsbestandenen Erdoberfläche zusammengefasst, da diese das theoretische Gerüst für die eigenen Messungen darstellen. Es folgt eine Diskussion der wichtigsten mikrometeorologischen Methoden zur Bestimmung vertikaler Stoff- und Energieflüsse zwischen Atmosphäre und Erdoberfläche. Da für die Messungen der vorliegenden Arbeit die Eddy - Kovarianzmethode Verwendung gefunden hat, wird diese besonders intensiv besprochen. Im dritten Teil werden Setup und Untersuchungsort der eigenen Messungen vorgestellt, im vierten und fünften Kapitel werden die Ergebnisse dieser Messungen beschrieben und diskutiert. 


\section{Theoretische Überlegungen zum Stoffaustausch zwischen Atmosphäre und Vegetation mit besonderer Berücksichtigung der Deposition von atmosphärischen Partikeln über Wäldern}

In diesem Kapitel werden allgemeine theoretische Grundlagen zum Stoffaustausch zwischen der Atmosphäre und der vegetationsbestandenen Erdoberfläche zusammengefasst. Zunächst werden die Systeme Atmosphäre und Vegetation in eigenen Abschnitten separat betrachtet, danach wird der Stoff- und Energieaustausch zwischen beiden Systemen erörtert. Die für die eigenen Untersuchungen der Partikeldeposition über Wäldern relevanten Punkte werden jeweils in Unterkapiteln detailliert erläutert.

\subsection{Die Atmosphäre}

Die Atmosphäre ist die Gashülle der Erde. Sie enthält neben ihren Hauptbestandteilen Stickstoff (molekular), Sauerstoff (molekular), Argon, Wasserdampf sowie geringen Mengen verschiedener Spurengase (wie z.B. Kohlendioxid, Neon, Helium, Ozon, Schwefeldioxid, Stickoxiden) flüssige und feste Schwebstoffe im Größenbereich von ca. 0,01 bis $100 \mu \mathrm{m}$ Durchmesser, die als "atmosphärische Partikel" bezeichnet werden und im Mittelpunkt der vorliegenden Arbeit stehen. Diese Schwebstoffe - etwa 1.000 bis 10.000 Teilchen pro $\mathrm{cm}^{3}$ Luft - beeinflussen den Strahlungshaushalt der Atmosphäre sowie die Prozesse der Wolkenund Niederschlagsbildung in der Troposphäre. Sie sind für die globalen Kreisläufe chemischer Elemente sowie für die Stoffhaushalte von Ökosystemen von großer Bedeutung und wirken darüber hinaus in vielfältiger Art und Weise auch auf die menschliche Gesundheit und das menschliche Wohlergehen ein (Abschnitt 1.1.1).

Die Atmosphäre wird vertikal in verschiedene Schichten eingeteilt, wobei je nach Art der Betrachtung unterschiedliche Einteilungskriterien zur Geltung kommen. Für mikrometeorologische Untersuchungen, wie die Bestimmung des Partikelflusses zur Erdoberfläche, ist vor allem das Konzept der "Planetarischen Grenzschicht" (planetary boundary layer - PBL) von großer Bedeutung. Mit diesem Konzept besitzen Mikrometeorologen eine eigene, für ihre Untersuchungen angepasste Modellvorstellung der Atmosphäre, die sich von denjenigen der Klimatologen einerseits und der Landschaftsökologen andererseits unterscheidet. Ein tieferes Verständnis der Prozesse innerhalb der PBL ist erforderlich, um die in der Mikrometeorologie angewandten Methoden zur Erfassung vertikaler Stoffflüsse und deren Grenzen bewerten zu können (Abschnitt 1.1.2).

Für großräumige, klimatologische Untersuchungen werden Bereiche der Atmosphäre mit gleichen (ähnlichen) Eigenschaften wie Temperatur und Wasserdampfgehalt üblicherweise als "Luftmasse" bezeichnet. Für kleinräumige, mikrometeorologische Untersuchungen wird 
Theoretische Überlegungen zum Stoffaustausch zwischen Atmosphäre und Vegetation

dagegen ein betrachteter Ausschnitt der Atmosphäre gedanklich in kleinere Einheiten (Luftquanten, Luftpakete) unterteilt. Es wird angenommen, dass sich diese Luftpakete innerhalb der Atmosphäre turbulent bewegen und untereinander sowohl Spurenstoffe als auch Energie (fühlbare Wärme, latente Energie) austauschen. Auf Grund von Stoff- bzw. Energieunterschieden innerhalb der Atmosphäre entstehen mit Hilfe dieser Prozesse gerichtete Stoff- und Energieflüsse, die häufig durch Quellen/Senken auf der Erdoberfläche angetrieben werden. Der in der vorliegenden Arbeit untersuchte Partikeltransport zwischen Atmosphäre und der Vegetation von Wäldern ist einer dieser Stoffflüsse (Kapitel 1.3).

Die Atmosphäre als Gashülle der Erde ist ein offenes System, das einer ständigen Dynamik unterworfen ist. Diese Dynamik resultiert aus den unterschiedlichen physikalischen und chemischen Eigenschaften der Erdoberfläche (Wasser und Land, Berg und Tal) sowie der zeitlichen und räumlichen Variabilität der Einstrahlungsintensität der Sonne auf die Erdoberfläche. Deshalb zeigen insbesondere im Bereich der PBL Lufttemperaturen, Windgeschwindigkeiten, Spurenstoffkonzentrationen und andere Parameter mit typischen Zeitskalen von wenigen Sekunden (turbulenter Transport), 24 Stunden (Tagesgang) und 365 Tagen (Jahresgang) ein ganzes Spektrum von Fluktuationen. So ist davon auszugehen, dass ein bestimmter Zustand der Atmosphäre an einem bestimmten Ort der Erde und zu einem bestimmten Zeitpunkt als einmalig und als nicht reproduzierbar anzusehen ist. Dieser Umstand schränkt die Aussagekraft einzelner meteorologischer Messungen bzw. Messreihen erheblich ein und stellt für die Forschenden Schwierigkeit und Herausforderung zugleich dar.

Vereinfacht lässt sich der Zustand der Atmosphäre jederzeit durch sieben Variablen beschreiben: der longitudinalen, der lateralen und der vertikalen Komponente des Windvektors $u, v$ und $w$; der Luftdichte $\rho$; der absoluten Temperatur $T$; der spezifischen Feuchte $q$ und den Luftdruck $p$. Die räumliche und zeitliche Abhängigkeit dieser Variablen kann durch ein System von sieben gekoppelten partiellen Differentialgleichungen beschrieben werden: den drei Bewegungsgleichungen (Navier-Stokes-Gleichungen, Impulserhalt), den Kontinuitätsgleichungen für Luft- und Wassermoleküle (Massenerhaltung), der Energiebilanzgleichung und dem allgemeinen Gasgesetz. Für die Lösung dieses Gleichungssystems ist die genaue Kenntnis der Anfangs- und Randbedingungen notwendig, so dass eine Lösung nur in Ausnahmefällen möglich ist.

\subsubsection{Detailbetrachtung Atmosphäre: Atmosphärische Partikel}

Unter dem Begriff der "atmosphärischen" bzw. "luftgetragenen Partikel" fasst man alle in der Atmosphäre suspendierten festen und flüssigen Schwebstoffe zusammen. Im Gegensatz hierzu wird die Gesamtheit aller in einem Luftvolumen enthaltenen festen und flüssigen Partikel, also die gesamte Fraktion der in der Atmosphäre kondensierten Materie, gemeinsam mit der sie umgebenden Luft als "atmosphärisches Aerosol"" (SchMAUSS \& WigAND, 1929) bezeichnet. Umgangssprachliche Bezeichnungen, wie "Dunst", "Rauch" oder "Nebel”, stehen

\footnotetext{
${ }^{2}$ Aerosol (griech./lat.): in Luft gelöst
} 
Theoretische Überlegungen zum Stoffaustausch zwischen Atmosphäre und Vegetation

in Zusammenhang mit einer qualitativen Aerosol - Charakterisierung von Luftmassen, soweit diese mit dem menschlichen Auge möglich ist.

\subsubsection{Physikalische und chemische Charakterisierung luftgetragener Partikel}

Einzelne, luftgetragene Partikel können mit verschiedenen Eigenschaften charakterisiert werden, wie z.B. der geometrischen Form, Partikelgröße, Lebensdauer, freien Weglänge, stofflichen Zusammensetzung und der Dichte. Eine umfassende Charakterisierung von Partikelkollektiven erfordert demgegenüber statistische Angaben zur Gesamtkonzentration, oberfläche, -volumen und -masse sowie Angaben zur Größenverteilung (“Aerosolspektrum”), zur Massenkonzentration und zum mittleren Durchmesser der in einem Luftvolumen enthaltenen Partikel. Im folgenden werden einige der genannten Eigenschaften diskutiert.

Die geometrischen Formen luftgetragener Partikel sind vielgestaltig und unregelmäßig. Aus praktischen Gründen wird jedoch häufig eine vereinfachte kugelförmige Geometrie angenommen. Eine mögliche Begründung für diese Annahme ist, dass Partikel mit wasserlöslichen Anteilen bei ausreichend hoher Feuchtigkeit in hoch konzentrierte Lösungströpfchen übergehen, welche eine annähernde Kugelform haben (WARNECK, 2000).

Der praktische Vorteil einer angenommen Kugelform ist zweifelsohne die Möglichkeit, die Größe luftgetragener Partikel in einfacher Weise über den Durchmesser beschreiben und die Partikel in dieser Weise klassifizieren zu können. Dabei wird unter dem Partikeldurchmesser ein sogenannter “Äquivalentdurchmesser" verstanden. Eine mögliche, von messverfahrenstechnischen Fragen unabhängige Definition für diesen ist der Durchmesser einer gedachten idealen Kugel mit dem selben Volumen wie das des betrachteten Partikels (z.B. WINKLER, 2000).

Partikel des atmosphärischen Aerosols umfassen ein enormes Größenspektrum von wenigen Nanometern bis hin zu mehreren Mikrometern Durchmesser. Partikel mit einem Radius $r<0,1 \mu \mathrm{m}$ werden in der Literatur als Aitkenkerne ("Aitken nuclei"), Partikel mit $r>0,1 \mu \mathrm{m}$ und $r<1 \mu \mathrm{m}$ als Große Partikel ("large particle") und Partikel mit $r>1 \mu \mathrm{m}$ als Riesenpartikel ("giant particles") bezeichnet. Daneben ist auch eine Einteilung in "coarse" $(r>1 \mu \mathrm{m})$, "fine" $(20 \mathrm{~nm}<r<1 \mu \mathrm{m})$ und "ultrafine" $(r<20 \mathrm{~nm})$ gebräuchlich (JAENICKE, 1993; MÄKELÄ ET AL., 1997).

Die Einteilung in Größenbereiche spiegelt die wichtige Beobachtung wieder, dass in bestimmten Größenklassen einzelne Prozesse besonders wirksam - oder nicht wirksam - sind und dass bestimmte chemische Bestandteile in jeweils unterschiedlichen Anteilen vorhanden sind. Beispielsweise sind Riesenpartikel im Verhältnis zu Großen Partikeln und Aitkenkernen in besonders starker Weise dem Einfluss der Sedimentation ausgesetzt, während die letzteren vor allem den Mechanismen der Brownschen Molekularbewegung unterliegen. Die Wirksamkeit einzelner, bestimmter Prozesse wiederum beeinflusst wesentlich die individuelle Lebensdauer von Partikeln in der Atmosphäre, die deshalb für die einzelnen Größenbereiche mit Werten zwischen wenigen Sekunden und mehreren Tagen entsprechend unterschiedlich groß ausfällt. 
Theoretische Überlegungen zum Stoffaustausch zwischen Atmosphäre und Vegetation

Das absolute Konzentrationsmaximum (das Maximum der Teilchenanzahl in einem Einheitsradiusintervall) befindet sich typischerweise im Bereich der Aitkenkerne bei einem Radius von etwa 0,01 bis $0,1 \mu \mathrm{m}$. Oberhalb von $r>0,1 \mu \mathrm{m}$ nimmt die Partikelkonzentration mit der Größe der Partikel rasch in Form einer Potenzfunktion ab. Partikel mit einem Radius $>0,25 \mu \mathrm{m}$ tragen nicht mehr nennenswert zur Gesamtpartikelanzahl bei, Partikel mit einem Durchmesser von mehr als $10 \mu \mathrm{m}$ werden in der Atmosphäre nur noch selten beobachtet (HaAtaja \& Vesala, 1997; Delany ET AL., 1967). Eine obere Grenzgröße des atmosphärischen Aerosols ist allerdings nicht bekannt (JAENICKE \& JUNGE, 1967). Unterhalb des Konzentrationsmaximums zeigt die Partikelgrößenverteilung eine besonders deutliche zeitliche Variabilität sowie eine starke Abhängigkeit von meteorologischen und chemischen Randbedingungen innerhalb eines Luftvolumens als auch von dessen Geschichte (MÄKELÄ ET AL., 1997).

In Zusammenhang mit der Herkunft von Luftmassen lassen sich nach JAENICKE (1993) für Mitteleuropa grob fünf Aerosoltypen unterscheiden, denen jeweils ein charakteristisches Partikelspektrum zugeordnet werden kann: "Reinluft", "kontinentale Reinluft", "ländliche Luft", "Stadtluft" und "maritime Luft". Erwartungsgemäß finden sich in der Abwindfahne städtischer Gebiete höhere Partikelkonzentrationen (etwa eine halbe bis eine Größenordnung mehr) als in ländlicher Luft, die wiederum stärker belastet ist als Reinluft. Eine allgemeinere Klassifizierung des atmosphärischen Aerosols lieferte bereits JUNGE (1963), der auf globaler Ebene zwischen "continental", "maritime" und "tropospheric background aerosol" unterschied.

Luftgetragene Partikel bestehen häufig sowohl aus fester Materie als auch aus flüssigem Wasser. Der Flüssigwasseranteil variiert dabei in Abhängigkeit von der relativen Feuchtigkeit der umgebenden Luft - insbesondere dann, wenn Partikel lösliche und hygroskopische Substanzen enthalten. Mit der Veränderung des Flüssigwasseranteils als Folge einer steigenden oder fallenden relativen Feuchte verändern sich - in Abhängigkeit von der chemischen Zusammensetzung und des Trockenradius - die Größe einzelner Partikel unter Umständen dramatisch (FITZGERALD, 1975). Gleichzeitig werden weitere Eigenschaften modifiziert, wie insbesondere die Lichtstreuung der Partikel, die geometrische Form, die Depositionsgeschwindigkeit und die Dichte der einzelnen Partikel. Letztere schwankt für kontinentale Luftmassen bei relativen Luftfeuchten bis $75 \%$ zwischen 1,8 und $3 \mathrm{~kg} / \mathrm{dm}^{3}$, während sie bei höheren Luftfeuchten auf etwa $1 \mathrm{~kg} / \mathrm{dm}^{3}$ reduziert werden kann (HÄNEL \& THUDIUM, 1977; WARNECK, 2000).

Bei sehr hoher relativer Luftfeuchtigkeit gehen Partikel in Nebel- oder Wolkentröpchen über. Letztere werden - getrennt von den atmosphärischen Partikeln - als separates System behandelt, obwohl sie im Prinzip in gleicher Art und Weise als stabile Suspension (flüssiger) Schwebstoffe in der sie umgebenden Luft angesehen werden können. Auf Grund des fließenden Übergangs kann keine eindeutige Grenze benannt werden; insbesondere existieren auch bei den Partikelgrößen Überlappungen zwischen den beiden Systemen (WARNECK, 2000). 
Theoretische Überlegungen zum Stoffaustausch zwischen Atmosphäre und Vegetation

Die chemische Zusammensetzung des atmosphärischen Aerosols ist abhängig von den Quellen der Partikelproduktion sowie den Umwandlungsprozessen, denen das betrachtete Aerosol unterliegt. So sind typische Bestandteile der durch Winderosion aufgewirbelten Bodenteilchen Silizium, Calcium, Eisen und Titan, während Meersalzpartikel hohe Gehalte an $\mathrm{Cl}^{-}$und $\mathrm{Na}^{+}$enthalten. Ein Ensemble von vielen Partikelteilchen kann eine sehr heterogene chemische Zusammensetzung aufweisen. Aber auch für die Zusammensetzung eines einzelnen Partikels ist schon ein Gemisch sehr vieler Substanzen und chemischer Elemente möglich. Diesem Phänomen wird mit dem sogenannten Mischkernkonzept Rechnung getragen, welches zwischen äußerer und innerer Mischung unterscheidet (ROEDEL, 1994; WINKLER, 2000).

Einzelne Inhaltsstoffe verteilen sich typischerweise über einen mehr oder weniger breiten, stoffspezifischen Größenbereich des Partikelspektrums. Zur Charakterisierung dieses Phänomens wurde der Begriff des mittleren Massendurchmessers (MMD) geprägt, unterhalb und oberhalb dessen sich jeweils 50\% der Masse der Substanz befinden. Das Konzept des MMDs erlaubt es, Resultate größenspezifischer Untersuchungen physikalischer Eigenschaften luftgetragener Partikel (wie die Ergebnisse der vorliegenden Arbeit) auf elementspezifische Fragestellungen zu übertragen.

\subsubsection{Der Lebenslauf luftgetragener Partikel: Entstehung, Umwandlung und Deposition von Partikeln des atmosphärischen Aerosols}

Aerosolpartikel unterliegen als Spurenstoffe in der Atmosphäre Quell-, physikalischen sowie chemischen Umwandlungs-, Transport- und Depositionsprozessen. Die physikalischen und chemischen Eigenschaften der Partikel werden im Laufe der Zeit durch aerosolphysikalische und meteorologische Prozesse kontinuierlich verändert. Die Aufenthalts- und Transportdauer der Partikel in der Troposphäre ist auf relativ kurze Zeiträume (einige Sekunden bis einige Tage) beschränkt, während Partikel in der Stratosphäre mehrere hundert Tage existieren können (JAENICKE, 1988).

Luftgetragene Partikel entstehen auf vielfältige Art und Weise. Zu den Bildungsmechanismen gehören die Dispergierung und Aufwirbelung von Bodenpartikeln, die Produktion von Seesalzpartikeln an der Meeresoberfläche durch das Zerplatzen von Luftblasen, das Abtrocknen von Wolkentropfen sowie die Partikelbildung aus der Gasphase durch homogene Kondensation übersättigter Dämpfe ("gas-to-particle conversion", GPC). Prominente Beispiele für die GPC sind die Oxidierung von $\mathrm{SO}_{2}$ und $\mathrm{NO}_{2}$ zu Schwefelsäure $\left(\mathrm{H}_{2} \mathrm{SO}_{4}\right)$ bzw. Salpetersäure $\left(\mathrm{HNO}_{3}\right)$. Wichtige anthropogene Quellen sind die Verbrennung fossiler Energieträger wie z.B. Kohle, Erdöl und Erdgas, die Eisen- und Stahlindustrie, die Zementproduktion sowie die Landwirtschaft. $\mathrm{Zu}$ den natürliche Ereignissen der Partikelbildung gehören Waldbrände und vulkanische Eruptionen, bei denen sowohl Aschepartikel als auch Vorläufergase für die GPC emittiert werden (GRÄDEL \& CRUTZEN, 1994; WARNECK, 2000).

Die sich in der Atmosphäre befindlichen Partikel verändern ihre Größe sowohl durch die Zusammenlagerung mehrerer Partikel ("Koagulation”) als auch durch die Anlagerung von 
Theoretische Überlegungen zum Stoffaustausch zwischen Atmosphäre und Vegetation

Gasmolekülen an vorhandene Partikel ("heterogene Kondensation"). Bei beiden Prozessen kommt es zu einer Verschiebung des Größenspektrums zu Gunsten der großen Partikel auf Kosten der Aitkenkerne. Darüber hinaus werden Partikel auch durch die Bildung und Wiederverdampfung von Wolkentröpfchen modifiziert.

Während ihres Aufenthaltes in der Atmosphäre können Partikel horizontal weite Distanzen zurücklegen und über globale Strecken verfrachtet werden. Dies führt dazu, dass feste und flüssige Schwebstoffe ein allgemeines Merkmal der unteren Atmosphäre sind und durchaus nicht auf Regionen mit Partikelquellen beschränkt bleiben (WARNECK, 2000; AHONEN ET AL., 1997).

Die Lebensdauer mittelgroßer und großer Partikel wird durch die Nasse und Trockene Deposition begrenzt, also durch die Ablagerung bzw. Abscheidung der Partikel auf und an den Strukturen der Erdoberfläche (Vegetation, Erdboden, Wasseroberflächen). Die Lebensdauer kleiner und ultrafeiner Partikel wird dagegen vor allem durch Koagulation und heterogene Kondensation eingeschränkt. Die betroffenen Partikel verlieren in diesen Fällen zwar ihre eigenständige Existenz, ihre Inhaltsstoffe verbleiben aber als Teil größerer und längerlebiger Partikel in der Atmosphäre. Partikel mit einem Durchmesser zwischen 0,01 und $0,1 \mu \mathrm{m}$ werden von Senkenprozessen nur schwer erfasst, was zu dem bereits beschriebenen Konzentrationsmaximum führt. Die kürzeste individuelle Lebensdauer weisen ultrafeine Partikel und die sehr rasch sedimentierenden Riesenpartikel mit Radien $>10 \mu \mathrm{m}$ auf.

\subsubsection{Auswirkungen des atmosphärischen Aerosols auf das Klima}

Luftgetragene Partikel besitzen geeignete Oberflächen für die Anlagerung von Molekülen und Ionen aus ihrer Umgebung. Sie fungieren in der Atmosphäre als Kondensationskerne und dienen darüber hinaus allgemein als Medien, auf denen wichtige chemische Reaktionen ablaufen können (GRÄDEL \& CRUTZEN, 1994). Weiter trüben Aerosolteilchen die Atmosphäre, so dass die Atmosphäre kurz- und langwellige Strahlung stärker reflektiert, streut oder absorbiert, wobei neben der Partikelgröße die Anzahldichte und die Art der Teilchen für die Stärke und die Art der Beeinflussung maßgebend ist (WINKLER, 2000).

Über diese physikalischen Eigenschaften greift das atmosphärische Aerosol im regionalen wie im globalen Maßstab in komplizierter und bisher noch längst nicht im Detail bekannter Weise in das Klimageschehen ein, indem es zum einen den Strahlungshaushalt der Atmosphäre und zum zweiten die Wolkenbildung beeinflusst und damit sowohl den Wärmehaushalt als auch den Wasserhaushalt der Erde modifiziert.

Während es heute bereits zahlreiche Hochrechnungen der Effekte für Treibhausgasszenarien gibt, existieren nur erste grobe Versuche, auch den Einfluss der Aerosole exakt zu bestimmen (GRAßL, 1999). Allgemein wird dabei zwischen der direkten Klimawirkung (verstärkte Reflektion, z.T. auch Absorption des kurzwelligen Sonnenlichtes) und der indirekten Wirkung (Einfluss auf die Wolkenbildung) des globalen Aerosols unterschieden. Sowohl die direkten als auch die indirekten Klimawirkungen führen für sich alleine betrachtet zu einem 
Theoretische Überlegungen zum Stoffaustausch zwischen Atmosphäre und Vegetation

sogenannten "Anti-Treibhauseffekt" und damit tendenziell $\mathrm{zu}$ einer Kompensation des anthropogen verursachten Treibhauseffektes (CHARLSON ET AL., 1992).

Eine zuverlässige, modellhafte Abschätzung des "Aerosol-Klimaeffektes" setzt neben der Kenntnis der optischen Eigenschaften von luftgetragenen Partikeln die Modellierung der Verteilung von Aerosolpartikeln und damit die Modellierung aller Quell-, Transport-, Umwandlungs- und Depositionsprozesse voraus. Ein Beitrag hierzu soll mit der vorliegenden Arbeit geleistet werden.

\subsubsection{Messtechnische Erfassung von Partikeln}

Atmosphärische Partikel können auf Impaktorenplatten abgeschieden, auf Filtern gesammelt oder mit Hilfe optischer Messgeräte erfasst werden (Tabelle 1). Die Gesamtanzahl der in einem Luftvolumen gefundenen Teilchen ist stark vom berücksichtigten Größenbereich und dem Zählwirkungsgrad (Verhältnis der Anzahl der detektierten Teilchen zur Anzahl der tatsächlich in einem Luftvolumen vorhandenen Partikel) des eingesetzten Messverfahrens abhängig. Bis heute gibt es kein einzelnes Verfahren, mit dem die Partikelkonzentration über das gesamte Größenspektrum des atmosphärischen Aerosols als Funktion der Partikelgröße bestimmt werden kann. Eine Übersicht zu gängigen Messtechniken geben WILLEKE \& BARON (1993).

Tabelle 1: Wichtigste Messverfahren zur Bestimmung der Konzentration luftgetragener Partikel (nach: WARNECK, 2000; ergänzt)

\begin{tabular}{|l|c|l|l|}
\hline \multicolumn{1}{|c|}{ Messverfahren } & $\begin{array}{c}\text { Partikelgröße } \\
(\mu \mathrm{m})\end{array}$ & \multicolumn{1}{|c|}{ Bestimmung von } & \multicolumn{1}{|c|}{ Theorie } \\
\hline Filter & $>0,01^{\mathrm{b})}$ & Gesamtmasse $^{\mathrm{c})}$ & (direktes Verfahren) \\
\hline Impaktorplatten & $>0,1$ & Masseverteilung & $\begin{array}{l}\text { MARPLE \& WILLEKE } \\
(1976)\end{array}$ \\
\hline Streulichtzähler & $>0,3$ & Anzahlverteilung & MIE (1908) \\
\hline Kondensationskernzähler & $0,005-0,8$ & Anzahl oder Volumen $^{\text {d) }}$ & AITKEN (1923) \\
\hline
\end{tabular}

a) Durchmesser; ${ }^{\text {b) }}$ abhängig vom Filtertyp und den zur Verfügung stehenden Analyseverfahren; ${ }^{\text {c) }}$ bei Auszählung unter dem Mikroskop auch Anzahlverteilung; ${ }^{\text {d) }}$ in Kombination mit einem Größen-Fraktionierer (electric mobility analyzer) als Anzahl- bzw. Volumenverteilung

Zeitlich hoch aufgelöste Messungen der Partikelkonzentration - wie sie für die Eddy Kovarianzmethode notwendig sind (vgl. Kapitel 2.2.2) - sind ausschließlich mit optischen Messverfahren möglich. Hier unterscheidet man Verfahren, die Partikelgrößen und -konzentration am Einzelpartikel bestimmen (Streulichtzähler, Aerosolspektrometer, Laufzeitspektrometer) von solchen, die die Eigenschaften am Partikelkollektiv untersuchen (Streulichtphotometer, Extraktionsphotometer, Fraunhoferbeugungsspektrometer). Im Gegensatz zur Kollektivuntersuchung werden bei der Einzelpartikelanalyse die Teilchen einzeln 
Theoretische Überlegungen zum Stoffaustausch zwischen Atmosphäre und Vegetation

einem optisch abgegrenzten Messvolumen zugeführt. Die Größe jedes Teilchens wird mit je einer separaten Messung bestimmt, so dass eine hohe Auflösung des Partikelspektrums realisiert werden kann. Im Vergleich zur Kollektivuntersuchung kann bei der Einzelpartikelanalyse pro betrachteten Zeitintervall jedoch nur ein relativ kleines Luftvolumen untersucht werden Deshalb sind bei diesem Messprinzip die Aussagen zur Partikelkonzentration größerer Luftvolumina im Vergleich zu Kollektivuntersuchungen insbesondere für zeitlich hochaufgelöste Messungen durch eine vergleichsweise hohe statistische Unsicherheit gekennzeichnet.

Da die Partikelgröße bei allen Verfahren (außer dem Mikroskop) indirekt über verschiedene physikalische Partikeleigenschaften erfasst wird, wird als Maß für die Partikelgröße häufig ein "Äqivalenzdurchmesser" angegeben, dessen Definition letztendlich vom jeweiligen Messverfahren abhängt. Typischerweise werden entweder optische Eigenschaften (z.B. "Streulichtäquivalentdurchmesser") oder die Beweglichkeit des Partikels (“Aerodynamischer Durchmesser") betrachtet. In der Regel beruhen diese indirekten Vorgehensweisen auf Vergleichsmessungen mit künstlichen Partikeln (Testaerosolen), deren Eigenschaften unter Umständen nicht vollständig mit denjenigen der betrachteten atmosphärischen Partikel übereinstimmen. Größere Partikel können in aufwendigen und wenig standardisierten Methoden auch direkt unter dem (Elektronen-)Mikroskop untersucht und vermessen werden. Hierbei ist jedoch davon auszugehen, dass die auf diese Art und Weise bestimmte Größe eines betrachteten Partikels im getrockneten Zustand nicht der ursprünglichen Größe des natürlicherweise mit Wassermolekülen angelagerten Teilchens in der Atmosphäre entspricht.

Beim Ansaugen der Probenluft in bzw. durch einen Impaktor, Filter oder Analysator müssen besondere Vorkehrungen getroffen werden. So müssen die Partikel isokinetisch erfasst werden, was entweder einer ständigen Regulierung der Ansauggeschwindigkeit oder des Ansaugquerschnittes gleichkommt. Die Ansaugvorrichtung muss möglichst aus Edelmetall sein, um Partikelverluste an den Wänden als Folge von elektrostatischen Effekten, wie diese beispielsweise für Teflon möglich sind, zu vermeiden (GALLAGHER ET AL., 1997). Darüber hinaus soll der Ansaugweg möglichst kurz, turbulenz- und impaktionsfrei gehalten werden ${ }^{3}$.

\subsubsection{Detailbetrachtung Atmosphäre: Die Planetarische Grenzschicht}

Die Atmosphäre wird vertikal in Schichten eingeteilt, wobei je nach Art der Betrachtung unterschiedliche Einteilungskriterien zur Geltung kommen. Ein in der Meteorologie weit verbreitetes Prinzip ist die Einteilung der Atmosphäre nach ihrem vertikalen Temperaturgradienten in "Troposphäre", "Stratosphäre", "Mesosphäre" und "Thermosphäre". Die Mikrometeorologie beschränkt sich jedoch auf das Studium der bodennahen Schichten der Atmosphä-

\footnotetext{
${ }^{3}$ Man beachte die Problematik, dass für die Konzentrationsmessung ein turbulenzfreies Ansaugen der Probenluft gefordert wird, um mögliche Partikelverluste zu minimieren, dagegen für die Eddy - Kovarianzmethode ein turbulentes Regime erwünscht wird, um ein Verschmieren der Konzentrationsfluktuationen zu vermeiden (vgl. Kapitel 2.2.4, Dämpfungskorrektur).
} 
re. Deshalb ist für sie vor allem das Konzept der "Planetarischen Grenzschicht" von Bedeutung (Abb. 1).

Als Planetarische Grenzschicht (planetary boundary layer - PBL) wird in der Mikrometeorologie die unterste, 100 bis 3000 Meter mächtige Schicht der Troposphäre bezeichnet. Im Gegensatz zu der darüber liegenden freien Atmosphäre wird sie in starkem Maße durch die Prozesse des vertikalen Austausches von Impuls, Wärme, Wasserdampf und Spurenstoffen zwischen Erdoberfläche und Atmosphäre geprägt; in Folge von Windscherung und thermischer Konvektion entwickelt sich hier eine turbulente Luftströmung. Ihre Obergrenze wird durch jene Höhe definiert, in der der Windvektor mit zunehmendem Abstand von der Erdoberfläche erstmals in die Richtung des geostrophischen Windes weist. Diese Obergrenze ist weder räumlich noch zeitlich konstant und im Falle von Windstille und hochreichender labiler Schichtung zudem nicht eindeutig ausgeprägt. Mächtigkeit und Struktur der Grenzschicht weisen über Land tägliche und jahreszeitliche Schwankungen auf (DWD, 1987; STULL, 1988).

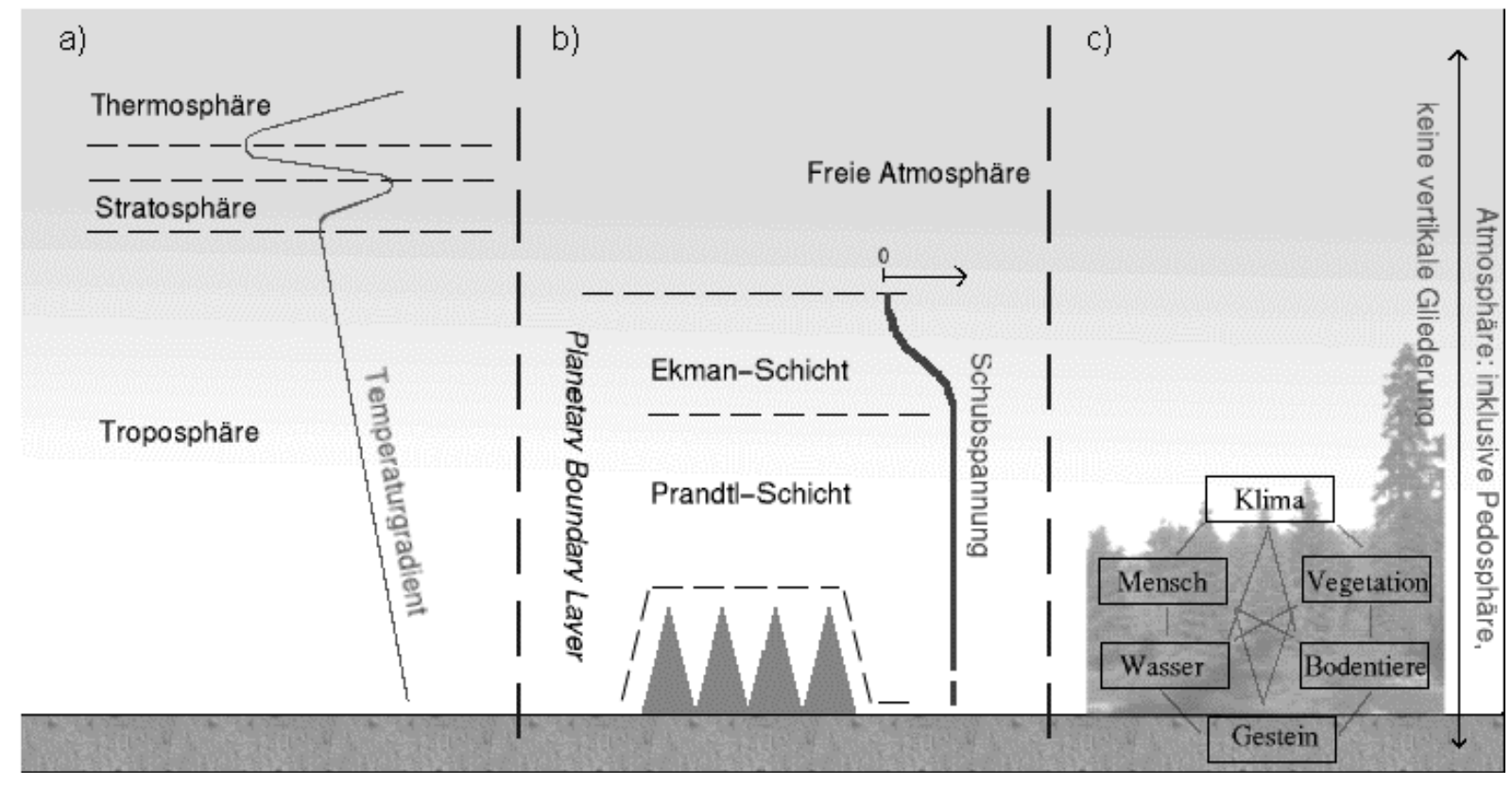

Abb. 1: Vertikale Gliederung der Atmosphäre: meteorologische (a), mikrometeorologische (b) und landschaftsökologische (c) Betrachtung im Vergleich. Erläuterungen siehe Text Abschnitt 1.1.2 und 1.2.1; nach: ROEDEL (1994), VOGT (1995) und LESER ET AL. (1997); verändert

Turbulente Strömungen, wie sie in der PBL entstehen, sind im Gegensatz zu laminaren Strömungen stochastische, nicht stationäre (hier: Luft-) Bewegungen, die durch unregelmäßige, räumliche und zeitliche Schwankungen im Geschwindigkeitsfeld gekennzeichnet sind. Sie können als das Durchlaufen von Turbulenzelementen bzw. Wirbeln (engl. eddies) verstanden werden, die sich in der Planetarischen Grenzschicht über Perioden von Bruchteilen einer Sekunde bis zu etwa einer Stunde erstrecken. Die Abmessungen dieser Elemente werden mit $10^{-2}$ bis $10^{3}$ Meter angegeben. Generell nimmt die Größe der eddies mit 
Theoretische Überlegungen zum Stoffaustausch zwischen Atmosphäre und Vegetation

zunehmender Nähe zum Boden ab, so dass die kleineren Turbulenzelemente bis zu etwa 20 Meter (mit Perioden < 1,2 Minuten) in unmittelbarer Nähe über der Vegetation anzusiedeln sind. Insgesamt hängt der Charakter und die Intensität der Turbulenz von der Windgeschwindigkeit, der Beschaffenheit der Unterlage und der herrschenden Schichtung der Atmosphäre ab (LILJEQUiST \& CEHAK, 1979; PICHLER, 1986).

Ein Teil der Turbulenzen in der PBL wird durch die Bodenreibung der Erdoberfläche verursacht. Man bezeichnet diesen Anteil auch als dynamische oder mechanische Turbulenz. Sie entsteht, wenn eine horizontale, zunächst laminare Windströmung in der Atmosphäre an ihrer Untergrenze durch die Rauhigkeit der Erdoberfläche abgebremst wird. Auf Grund der freien Beweglichkeit der Luftquanten überwälzen die ungebremsten (oberen) Stromteile die langsamer vorwärtskommenden (unteren) Stromteile, die ihrerseits auf der Rückseite von Walzen vom Boden abgehoben werden. Es entwickeln sich so in der ursprünglich horizontalen Windströmung vertikale Bewegungen, die Strömung wird turbulent (WEISCHET, 1995).

Durch das Abbremsen der Luftmasse in Folge der Bodenreibung verliert diese in Bodennähe einen Teil ihres Impulses (ihrer Bewegungsenergie). Es entsteht so ein vertikaler Gradient von der Erdoberfläche zur Atmosphäre, der sich in Form eines logarithmischen Profils der horizontalen Windgeschwindigkeit ausdrückt und einen vertikalen Impulsfluss von der Atmosphäre zur Erdoberfläche zur Folge hat.

Zwischen den Schichten unterschiedlicher mittlerer Windgeschwindigkeit wird eine Schubspannung initiiert. Aus dieser Größe wird unter Verwendung der Luftdichte die sogenannte Schubspannungsgeschwindigkeit definiert, die im allgemeinen als Maß für die mechanische Turbulenz Verwendung findet ${ }^{4}$. Es gilt (STULL, 1988):

$$
\begin{aligned}
& u_{*}=\sqrt{\frac{\tau}{\rho}} \\
& \text { mit: } u_{*} \quad \text { Schubspannungsgeschwindigkeit }(\mathrm{m} / \mathrm{s}) \\
& \tau \text { Schubspannung }\left(\mathrm{N} / \mathrm{m}^{2}\right) \\
& \rho \text { Luftdichte }\left(\mathrm{kg} / \mathrm{m}^{3}\right)
\end{aligned}
$$

Am Tage wird die mechanische Turbulenz zusätzlich durch Vertikalbewegungen von Luftpaketen unterstützt, die an der Bodenoberfläche erwärmt worden sind bzw. zu dieser zurückkehren. Man spricht von thermischen Auftrieb bzw. von thermisch induzierter Turbulenz. Voraussetzung hierfür ist eine labile Schichtung der Grenzschicht, also eine Temperaturabnahme mit der Höhe, welche größer als der Betrag des adiabatischen Temperaturgradienten ist. Dieser beträgt für trockene Luft $0.0098 \mathrm{~K} \mathrm{~m}^{-1}$. Umgekehrt werden bei einer stabilen Luftschichtung Vertikalbewegungen unterdrückt, wodurch die mechanische Turbulenz eine Abschwächung erfährt (DWD, 1987).

\footnotetext{
${ }^{4}$ Nach STULL (1988) kann die mechanisch induzierte Turbulenz (MT) mit $-u^{\prime} w^{\prime} \cdot d U / d z$ beschrieben werden. Nach Gleichung (18) gilt $u^{\prime} w^{\prime}=u_{*}{ }^{2}$. Weiter gilt $d U / d z=u_{*} /(k \cdot z)$ für nahe neutrale Schichtungen bzw. für alle Höhen kleiner $L$ (Monin-Obuchov-Mischungslänge). Unter diesen Bedingungen ist MT also proportional zu $u_{*}^{3}$ und $u_{*}$ folglich ein Maß für MT. Vgl. hierzu auch STULL (1997).
} 
Das Verhältnis zwischen mechanischer Turbulenz und thermischem Auftrieb kann mit der Monin-Obuchov-Mischungslänge $L$ charakterisiert werden. Sie ergibt sich nach PANOFSKY \& DutTon (1984) aus:

$$
L=-\frac{u_{*}{ }^{3} c_{p} \rho T}{k_{a} g H\left(1+\frac{0.07}{\beta}\right)}
$$

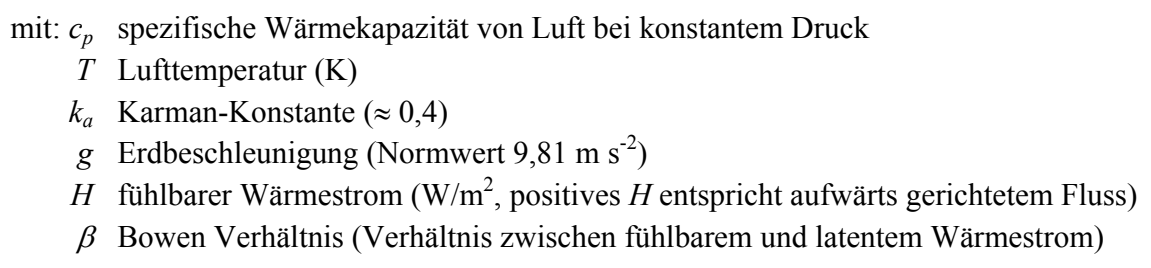

Es gilt $L<0$ für labile, $L>0$ für stabile Schichtungsbedingungen. Häufig wird der Term $z / L$ als Stabilitätsindex verwendet, wobei $z$ die Höhe über dem Erdboden ist. Bei stabiler Schichtung gilt dann folglich $z / L>0$, bei instabiler Schichtung $z / L<0$. Bei rauhen Oberflächen wird $z$ um eine Verdrängungsschichtdicke $d$ (displacement length) verringert.

Die in der Planetarischen Grenzschicht in Folge von Bodenreibung und thermischen Auftrieb entstehenden Turbulenzelemente spielen die entscheidende Rolle beim Austausch von fühlbarer Wärme und Spurenstoffen zwischen Erdoberfläche und Atmosphäre. Man spricht deshalb hier von einer turbulenten Diffusion der Stoffe bzw. von turbulenten Massen- und Energieflüssen.

Für detailliertere Betrachtungen ist es sinnvoll, die PBL vertikal weiter zu unterteilen in eine "molekular-viskose Unterschicht" bis etwa $1 \mathrm{~mm}$ Höhe über Boden bzw. Vegetation, eine "Prandtl-Schicht" bis 20-60 m, maximal auch bis 100 m Höhe, sowie eine "Ekman-Schicht" bis zur Obergrenze der PBL (Abb. 1). Für mikrometeorologische Messungen der vertikalen, turbulenten Austauschvorgänge in der PBL ist die Prandtl-Schicht (auch: surface layer, constant flux layer) entscheidend. Obwohl ihre Mächtigkeit nur rund $10 \%$ der gesamten PBL beträgt, erfolgt hier 70-80\% der reibungsbedingten Abnahme der Windgeschwindigkeit. In ihr ist die Schubspannung, die zwischen Schichten unterschiedlicher mittlerer Windgeschwindigkeit entsteht, von der Höhe unabhängig und gleich der Reibungskraft an der Bodenoberfläche (LILJEQUIST \& CEHAK, 1979). Auch für den Vertikalaustausch von Impuls, fühlbarer Wärme und Spurenstoffen können vertikale Variationen innerhalb des PrandtlSchicht vernachlässigt werden. Da dies nur für die Prandtl-Schicht, nicht jedoch für die Ekman-Schicht zutrifft, müssen (für die Erdoberfläche repräsentative) Flussmessungen immer in dieser Schicht durchgeführt werden.

Bei rauher Unterlage oder starker Geländegliederung wird der unterste Teil der PrandtlSchicht noch einmal gesondert betrachtet und als "Rauhigkeitsschicht" oder als "dynamische 
Theoretische Überlegungen zum Stoffaustausch zwischen Atmosphäre und Vegetation

Unterschicht" (roughness sublayer) bezeichnet. Hier sind die Austauschvorgänge von lokalen Begebenheiten beeinflusst und variieren in gleicher Höhe stark (FOKEN, 1990; VOGT, 1995).

\subsection{Die vegetationsbestandene Erdoberfläche aus meteorologischer Sicht}

\subsubsection{Allgemeine Charakterisierung der vegetationsbestandenen Oberfläche}

Im Gegensatz zu "Atmosphäre" ist "Vegetation" ein abstrakter Begriff, der die Gesamtheit aller pflanzlichen Organismen an einem Ort auf der Erde beschreibt. Für die gesamte Erde und gemeinsam mit der Fauna und den Mikroorganismen wird sie - als Pendant zur Atmosphäre - auch als Biosphäre bezeichnet. Die Biosphäre grenzt an die Atmosphäre und die Lithosphäre an, schließt aber gleichzeitig auch Teilbereiche dieser Sphären mit ein.

Betrachtet man einen bestimmten, abgegrenzten und einheitlichen Pflanzenbestand an einem Ort, wird dieser als Ökosystem charakterisiert. Dabei werden sowohl Tierwelt, Mikroorganismen als auch die physikalischen und chemischen Umweltbedingungen im System sowie die zahlreichen Wechselbeziehungen zwischen den einzelnen Komponenten untereinander mehr oder weniger detailliert in eine Betrachtung mit eingeschlossen.

Der Zustand einer einzelnen Pflanze kann nur mit sehr vielen Parametern sachgerecht beschrieben werden, wie z.B. Gattung und Art, Alter, genetische Besonderheiten, Größe, Masse, Form etc. Unmöglich ist es dagegen, einen Pflanzenbestand als Ganzes exakt zu beschreiben, wie z.B. einen Wald. Hier ist lediglich die Angabe von Summenparametern möglich, wie z.B. Angaben zur Biodiversität, zu vertretenen Pflanzenarten, zur Baumdichte, zur (oberirdischen) Pflanzenmasse, zur Gesamtstammdichte und zur Schichtstruktur. Da Pflanzenbestände immer dynamische Systeme sind, wäre für eine sachgerechte Beschreibung weiter die Quantifizierung von Veränderungsraten notwendig. Dies ist aber nicht zuletzt deshalb schwierig, weil hier kein klar definiertes Konzept zur Verfügung steht.

Mikrometeorologen beschreiben das komplexe System Vegetation häufig - im Vergleich zu Landschaftsökologen und Biologen - mit stark aggregierten, eindimensionalen Modellen. Sie betrachten einen Pflanzenbestand im wesentlichen als eine einzelne, horizontale, aktive Schicht biologischen Materials, die den Boden von der Atmosphäre abschirmt, Impuls absorbiert, $\mathrm{CO}_{2}$ sowie andere Spurenstoffe aufnimmt und abgibt, und Wassermengen in größeren Mengen transpiriert, als dies der Boden selbst tun würde. Darüber hinaus wird die Vegetation zusammen mit dem Boden als Strahlungsumsatzfläche gesehen, welche kurzwellige und langwellige Strahlung absorbiert, reflektiert oder emittiert und mit Hilfe turbulenter Wärmeflüsse Energie mit der Atmosphäre austauscht.

Ähnlich wie die Atmosphäre ist die Vegetation ein dynamisches System, dessen Eigenschaften sich auf unterschiedlichen Zeitskalen ständig verändern. Während einige dieser Veränderungen auch im Blickpunkt von meteorologischen Untersuchungen stehen, wie z.B. der Tages- und Jahresgang von Transpiration, Photosynthese oder Respiration, finden länger- 
Theoretische Überlegungen zum Stoffaustausch zwischen Atmosphäre und Vegetation

fristige Trends, wie genetische Mutationen, keinen oder nur einen beschränkten Niederschlag in den entsprechenden Studien. In diesem Sinne kann gesagt werden, dass Meteorologen die Vegetation - insbesondere wenn es sich um Wälder und Forste handelt - in ihren Untersuchungen als ein quasi stationäres System betrachten.

\subsubsection{Quantitative Beschreibung der vegetationsbestandenen Oberfläche aus mikrometeorologischer Sicht}

Aus mikrometeorologischer Sicht sind neben der Evapotranspirationsrate vor allem die Form, Größe und Anordnung der einzelnen Pflanzenteile die entscheidende Eigenschaften eines Pflanzenbestandes, da die Pflanzen als Rauhigkeitselemente und als Depositionsflächen fungieren, und damit turbulente Stoff- und Energieflüsse innerhalb der Atmosphäre beeinflussen.

Die Fähigkeit eines Pflanzenbestandes, der über ihm strömenden Luft kinetische Energie zu entziehen, wird mit der Rauhigkeitslänge $z_{0}$ quantifiziert. Diese gibt die Höhe an, bei der die in den Bestand hinein interpolierte mittlere horizontale Windgeschwindigkeit $\bar{u}$ den Wert Null erreicht. Für eine Wiese liegt der Betrag von $z_{0}$ zwischen 1 und $10 \mathrm{~mm}$ und für Wald bei 0,5 bis $3 \mathrm{~m}$ (ROEDEL, 1994). Demgegenüber gibt die bereits in Abschnitt 1.1.2 eingeführte Verdrängungsschicht $d$ die effektive Höhe des Bestandes für die Absorption des Impulses an. Die Größenordnung von $d$ beträgt im allgemeinen 70-80\% der Höhe des betrachteten Pflanzenbestandes (PANOFSKY \& DUTTON, 1984).

Für einen hypothetischen, gleichförmig gestalteten und horizontal homogenen Bestand wird angenommen, dass sowohl $z_{0}$ als auch $d$ konstant sind. Da aber die reale Vegetation aus unregelmäßigen und vor allem flexiblen Elementen besteht, hängen beide Parameter doch auch von der Windgeschwindigkeit ab (MONTEITH, 1975).

Der Blattflächenindex $L A I$ beschreibt das Verhältnis von gesamter Vegetationsoberfläche zur Grundfläche, welche als die vertikal projizierte, von der Vegetation bestandene Erdoberfläche definiert ist. Da die Oberfläche der Vegetationselemente im allgemeinen größer als die Grundfläche ist, ist der Blattflächenindex in der Regel größer als eins. Der Blattflächenindex ist ein wichtiger Parameter zur Beschreibung der Senkenstärke eines Pflanzenbestandes.

Neben den genannten Parametern können Pflanzenbestände mit einer Vielzahl weiterer Kenngrößen beschrieben werden, die jedoch für die vorliegende Arbeit keine Bedeutung haben und auf die deshalb hier nicht weiter eingegangen wird.

\subsection{Stoff- und Energieaustausch zwischen Atmosphäre und Erdoberfläche}

Atmosphäre und Vegetation sind über Prozesse des Stoff- und Energieaustausches miteinander verknüpft und beeinflussen sich deshalb wechselseitig. Beispielsweise reagieren 
Theoretische Überlegungen zum Stoffaustausch zwischen Atmosphäre und Vegetation

Pflanzen mit verändertem Wachstum auf Klimaänderungen, verändern sich die Artenzusammensetzungen von Ökosystemen bei modifizierten Randbedingungen, oder werden Ökotope bei Naturkatastrophen unter Umständen schwer beschädigt. Umgekehrt sind die drei langlebigen Treibhausgase Kohlendioxid, Methan und Distickstoffoxid (Lachgas) vor allem Produkte der Biosphäre. Pflanzenbestände regulieren die Evapotranspirationsrate und können im Extremfall über ihre Spaltöffnungen sogar "aktiv" die Höhe der PBL verändern und so die Bedeckung mit Schönwetterwolken "regeln" (GRAßL, 1999).

Die Prozesse des Stoff- und Energieaustausches zwischen Vegetation und Atmosphäre können den folgenden Vorgängen zugeordnet werden:

- dem Strahlungs- und Energieumsatz an der Erdoberfläche,

- dem Umsatz von atmosphärischen $\mathrm{CO}_{2}$ während der Photosynthese und der Respiration,

- der Deposition von Spurenstoffen (Gase, Partikel) und

- der Emission von Gasen und biogenen Partikeln in die Atmosphäre.

Die Umsatzraten der Einzelprozesse sind abhängig sowohl vom Zustand der Atmosphäre (Strahlungsregime, Spurenstoffkonzentration, Wasserdampfgehalt, Windgeschwindigkeit, Temperatur) als auch vom Zustand des Bodens und der Vegetation (Feuchte und Temperatur, Größe und Geometrie) sowie von der Natur der an den Prozessen beteiligten physikalischen und physiologischen Mechanismen (turbulenter Transport, Niederschlag, Diffusion). Die relative Bedeutung eines bestimmten Prozesses kann sich in Folge von Veränderungen innerhalb des Pflanzenbestandes, wie z.B. Alterung, oder in Folge von Veränderungen der Umwelt reversibel oder irreversibel verändern (MONTEITH, 1975).

Seit Mitte des 20. Jahrhunderts steht die Erfassung und Analyse des Stoff- und Energieaustausches zwischen Atmosphäre und Erdoberfläche im Forschungsfeld verschiedener Arbeitsgruppen von Agraringenieuren, Ökologen, Hydrologen und nicht zuletzt von Mikrometeorologen. Dabei wird entweder physiologisch-experimentell gearbeitet beispielsweise in Klimakammern, in der Regel dann mit Einzelpflanzen oder einzelnen Pflanzenteilen - oder beobachtend im Feld unter realen atmosphärischen Bedingungen. Während im ersteren Fall die experimentellen Randbedingungen kontrolliert und modifiziert werden können, verursacht die Dynamik der Atmosphäre im letzteren Fall stetig wechselnde Anfangs- und Randbedingungen, so dass hier im strengen Sinne nicht von Experimenten gesprochen werden kann. Die vorliegende Arbeit stellt mit ihren Messungen einen typischen Vertreter des beobachtenden Ansatzes mikrometeorologischer Messungen dar, bei denen die untersuchten Pflanzengesellschaften weder direkt noch indirekt manipuliert oder beschädigt werden $^{5}$.

\footnotetext{
${ }^{5}$ Es gilt offensichtlich für (fast) alle Aktivitäten der Meteorologie, dass diese Wissenschaft einen sehr freundlichen "Umgang" mit ihren Untersuchungsobjekten pflegt. Vgl. hierzu P.Seibert: 'Ist die Meteorologie eine feministische Musterwissenschaft ?" und E.Tamm: "Naturwissenschaft und Geschlechterrolle" in HEYMANN (1995).
} 
Theoretische Überlegungen zum Stoffaustausch zwischen Atmosphäre und Vegetation

\subsubsection{Detailbetrachtung Stoff- und Energieaustausch: Der Energieumsatz an der Erdoberfläche}

Während die Erde als Planet in Bezug auf Stoffe ein geschlossenes System ist, und diese deshalb auf der Erde in geschlossenen Kreisläufen zirkulieren, findet zwischen All und Erde ein ständiger Energieaustausch statt. Die Erde empfängt Energie in Form kurzwelliger solarer Strahlung und gibt sie als langwellige Erdstrahlung oder als kurzwellige reflektierte Solarstrahlung wieder ab. Da die Erde auf Dauer keine Energie speichern kann, muss die Differenz aus Ein- und Ausstrahlung für ein hinreichend langes Zeitintervall und den gesamten Planeten betrachtet Null sein.

Die Strahlungsbilanz zu einem bestimmten Zeitpunkt an einem konkreten Punkt der Erdoberfläche ist die Summe der momentanen kurzwelligen Strahlungsbilanz (Differenz aus Globalstrahlung und reflektierter kurzwelliger Strahlung) und der momentanen langwelligen Strahlungsbilanz (Differenz aus atmosphärischer Gegenstrahlung und terrestrischer Emission). Sie hängt im wesentlichen von Tages- und Jahreszeit, von der geographischen Breite und der Höhe über dem Meeresspiegel, von lokalen Reflexions- und Emissionsvermögen der Erdoberfläche sowie vom Spurenstoffgehalt der Atmosphäre ab und weist so erhebliche räumliche und zeitliche Variationen auf. In der Regel ist sie ungleich Null, so dass in Folge von Ein- und Ausstrahlung an der Erdoberfläche entweder ein Energiegewinn ("positive Strahlungsbilanz") oder ein Energieverlust ("negative Strahlungsbilanz") zu kompensieren ist.

Diese Kompensation erfolgt durch molekulare Wärmeleitung und turbulente Wärmeflüsse: für positive Strahlungsbilanzen von der Strahlungsumsatzfläche in die Atmosphäre und in den Boden hinein, für negative Strahlungsbilanzen zur Strahlungsumsatzfläche aus der Atmosphäre oder dem Boden heraus. In den Ozeanen ist zusätzlich auch der seitliche Transport von Wärme durch die Meeresströmungen möglich.

Die Energiebilanzgleichung für Landoberflächen kann vereinfacht wie folgt formuliert werden:

$$
R_{n}+G=L E+H
$$

$$
\begin{aligned}
\text { mit: } R_{n} & \text { Strahlungsbilanz } \\
G & \text { Bodenwärmestrom } \\
L E & \text { latenter Wärmestrom } \\
H & \text { fühlbarer Wärmestrom }
\end{aligned}
$$

Alle Terme können sowohl positive als auch negative Werte annehmen. Für die turbulenten Flüsse $\lambda E$ und $H$ beschreiben dabei negative Vorzeichen Energietransporte zur Strahlungsumsatzfläche hin, positive Vorzeichen dagegen Energietransporte von der Umsatzfläche weg. Für $R_{n}$ und $G$ gilt entsprechend die gegenteilige Vereinbarung.

Bei Vorhandensein von Vegetation ist für eine vollständige Charakterisierung des Energieumsatzes an der Erdoberfläche die Betrachtung einer weiteren Größe notwendig, die 
Theoretische Überlegungen zum Stoffaustausch zwischen Atmosphäre und Vegetation

die temporäre Speicherung von Energie innerhalb des Pflanzenbestandes beschreibt. Diese Größe wird als Bestandswärmestrom bezeichnet. Auch für den Fall des Vorhandenseins von Schnee- oder Eisdecken, für den Fall horizontaler Transporte von Wärme und Wasserdampf oder im Falle von Niederschlägen müssten zusätzliche Ausdrücke in die obige Gleichung eingeführt werden (WARNECKE, 1997; VOGT, 1995).

Die Summe aus Strahlungsbilanz, Bodenwärme- und ggf. Bestandswärmestrom wird im allgemeinen als verfügbare Energie bezeichnet. Aus der Energiebilanzgleichung (3) folgt, dass der Betrag der verfügbaren Energie der Summe aus fühlbaren und latenten Wärmestrom entsprechen sollte, wenn andere Energieströme vernachlässigt werden können. Kann dies mit den Daten einer meteorologischen Messung gezeigt werden, spricht man von der Schließung der Energiebilanz (energy balance closure), welche als Indiz für eine gute Qualität der Messdaten gilt.

In dem Maße, wie seit Anfang der 80er Jahre wesentlich verbesserte Messgeräte für mikrometeorologische Untersuchungen der turbulenten Energieströme zur Verfügung standen, wurde mehr und mehr offensichtlich, dass eine vollständige Schließung der Energiebilanz jedoch auch trotz sorgfältiger Fehlerabschätzungen häufig nicht erreicht werden kann (FOKEN, 1998). Als mögliche Ursachen hierfür werden Ungenauigkeiten bei der Bestimmung des langwelligen Strahlungsstroms und des latenten, turbulenten Wärmestroms (z.B. VoGT, 1995), Unsicherheiten über die Energiespeicherung im bodennahen Bereich der Atmosphäre und in der obersten Bodenschicht (z.B. BRAUD ET AL., 1993) sowie die Heterogenität der Unterlage und damit verbundene horizontale Flussdivergenzen (z.B. PANIN ET AL., 1996) vermutet. Generelles Problem ist die Unmöglichkeit, alle Größen der Energiebilanzgleichung an der Erdoberfläche unmittelbar an der Grenzfläche und damit in einer Höhe sowie wirklich für ein und dieselbe horizontale Bezugsfläche bestimmen zu können (FOKEN, 1998).

\subsubsection{Detailbetrachtung Stoff- und Energieaustausch: Spurenstoffdeposition}

Unter dem Begriff der Deposition ${ }^{6}$ von atmosphärischen Spurenstoffen versteht man die Ablagerung atmosphärischer Spurenstoffe in Form von Partikeln und Gasmolekülen auf Pflanzen-, Wasser- oder Bodenoberflächen. Es handelt sich um einen Prozess des Stoffaustauschs zwischen Atmosphäre und Erdoberfläche, bei dem Luftinhaltsstoffe aus der Atmosphäre in einen Pflanzenbestand oder ein Gewässer eingetragen werden. Spurenstoffdeposition ist deshalb immer sowohl mit einem Stoffeintrag in ein terrestrisches oder aquatisches Ökosystem hinein ("Nähr- und Schadstoffzufuhr", "natürliche Düngung") als auch mit einem Stoffaustrag aus der Atmosphäre ("Selbstreinigung der Atmosphäre") heraus verbunden und wirkt sich deshalb sowohl auf Prozesse in der Atmosphäre als auch auf Prozesse in den betroffenen Ökosystemen aus. In der Tat beziehen naturnahe Ökosysteme den allergrößten Teil ihrer Nähr- und Mineralstoffe aus dem atmosphärischen Spurenstoffeintrag,

\footnotetext{
${ }^{6}$ Deposition (lat. deponere): Hinterlegung, Niederlegung einer beweglichen Sache, hier: Ablagerung
} 
Theoretische Überlegungen zum Stoffaustausch zwischen Atmosphäre und Vegetation

wie MARQUES (1998) für einen Fichtenforst im Solling beispielhaft anhand der Elemente Schwefel und Stickstoff zeigt.

Idealerweise besteht zwischen dem Stoffeintrag und dem Stoffaustrag eines Ökosystems ein langfristig eingespieltes Gleichgewicht, so dass der Stoffvorrat konstant bleibt und der Zustand des Systems als stabil bezeichnet werden kann. In Folge anthropogener Emissionen haben sich aber die Konzentrationen verschiedener Spurenstoffe in der Atmosphäre gegenüber den natürlichen Verhältnissen so stark erhöht, dass die Nähr- und Schadstoffzufuhr aus der Atmosphäre für viele Ökosysteme massiv zugenommen hat, und damit die Stabilität dieser Systeme heute möglicherweise nicht mehr gegeben ist. Insbesondere beschleunigt der Eintrag sauer wirkender Spurengase Nährstoffausträge aus den Böden, als dessen direkte Folge u.a. Mangelerscheinungen bei Pflanzen beobachtet werden. Neben der Belastung durch Säureeinträge spielen zunehmend aber auch Stickstoffeinträge eine bedeutende Rolle, die den Base-/Säurezustand der Böden beeinflussen, ebenfalls auf den Nährstoffhaushalt einwirken und zu einer allgemeinen Eutrophierung führen (MEESENBURG ET AL., 1997).

Die Prozesse des Eintrags atmosphärischer Spurenstoffe werden entsprechend der Phasen, in denen die Stoffe in der atmosphärischen Grenzschicht vorkommen, der "Nassen Deposition" oder der "Trockenen Deposition" zugeordnet. Unter der Trockenen Deposition, die unabhängig von Niederschlagsereignissen stattfindet, versteht man die direkte Ablagerung von luftgetragenen Partikeln und Spurengasen auf Akzeptorflächen von Pflanzen und Boden. Als Nasse Deposition wird dagegen der Eintrag von im Niederschlag enthaltenen Spurenstoffen bezeichnet (RoEDEL, 1994). Im Gegensatz zur Nassen Deposition ist die Trockene Deposition von den physikalischen und chemischen Oberflächeneigenschaften des Ortes, auf dem deponiert wird, abhängig und wird somit in terrestrischen Ökosystemen insbesondere vom jeweiligen Vegetationstyp beeinflusst (WIMAN ET AL., 1990; GRAVENHORST ET AL., 2000).

Eine gewisse Sonderstellung nimmt die Deposition von Nebeltröpfchen ein, da diese zwar unabhängig von Niederschlagsereignissen ist, die Spurenstoffe andererseits aber in gelöster Form deponiert werden. Die Nebeldeposition (auch: "feuchte Deposition") stellt deshalb in gewisser Weise ein Bindeglied zwischen Nasser und Trockener Deposition dar, steht aber vom Abscheidemechanismus her betrachtet der Trockenen Deposition großer Partikel näher (IBROM, 1993, VONG \& KOWALSKI, 1995).

Ausreichend hohe Niederschläge vorausgesetzt, gilt für atmosphärische Partikel im mittleren Größenbereich mit Teilchenradien zwischen einigen Hundertsteln Mikrometer bis etwa 10 Mikrometer die Nasse Deposition als der effektivste Senkenprozess. Er begrenzt die Lebensdauer der betroffenen Partikel auf einige wenige Tage. Da die Hauptmasse der Partikel des atmosphärischen Aerosols der Troposphäre in diesem Größenbereich zu finden ist, stellt die mittlere Lebensdauer für die Ausscheidung durch die Nasse Deposition von etwa 4 bis 6 Tagen gleichzeitig ein gutes Maß für die Lebensdauer der Aerosolmasse überhaupt dar.

Mit zunehmender Partikelgröße verliert die Nasse Deposition ihren Charakter als dominierender Senkenprozess für atmosphärische Partikel. Für die Lebensdauer der Teilchen ist dann die 
Theoretische Überlegungen zum Stoffaustausch zwischen Atmosphäre und Vegetation

Trockene Deposition ausschlaggebend. Auch im unteren Größenbereich, unterhalb etwa $0,1 \mu \mathrm{m}$ Radius, nimmt die Effizienz der nassen Ausscheidung erst langsam, dann aber sehr rasch ab. Für die Lebensdauer der sehr kleinen Partikel wird die thermische Koagulation bestimmend, deren Effektivität in einem Gemisch verschieden großer Teilchen mit abnehmendem Partikelradius schnell zunimmt. Gasmoleküle werden durch die Nasse Deposition überwiegend erst dann aus der Atmosphäre entfernt, wenn sich infolge fortgeschrittener Oxidation ihre Löslichkeit bzw. Reaktivität in der kondensierten Phase so weit erhöht hat, dass sie dauerhaft in Partikel inkorporiert und mit diesen ausgeregnet werden (ROEDEL, 1994).

In den folgenden Abschnitten werden Mechanismen, Quantifizierung und messtechnische Erfassung der Trockenen Deposition luftgetragener Partikel detaillierter beschrieben. Auf die Nasse Deposition, die Nebeldeposition und die Ablagerung von Gasmolekülen soll dagegen nicht weiter eingegangen werden, da diese für die vorliegende Arbeit keine Bedeutung haben.

\subsubsection{Mechanismen der Trockenen Deposition luftgetragener Partikel}

Die Trockene Deposition luftgetragener Partikel ist das Ergebnis mehrerer Teilprozesse. Durch die Sedimentation und turbulente Diffusion werden die Partikel zunächst vertikal durch die Atmosphäre transportiert. Die molekular-viskose Unterschicht der PBL wird mit Hilfe von thermischer Diffusion und Sedimentation überwunden, die Anlagerung der Partikel an eine Akzeptorfläche erfolgt schließlich durch Impaktion und Adhäsion. Letzterer Prozess wird möglicherweise auch noch durch andere Mechanismen wie Elektrophorese und Thermophorese unterstützt (GALlagher ET AL., 1997; WyERS \& DUYZER, 1997). Die Wirksamkeit der einzelnen Mechanismen wird durch die Teilchengröße, die Akzeptoreigenschaften und die Luftbewegung bestimmt. Die jeweils dominanten Prozesse für Partikel $<0,1 \mu \mathrm{m}$ Durchmesser bzw. $>1 \mu \mathrm{m}$ Durchmesser sind in Tabelle 2 zusammengestellt.

Tabelle 2: Dominante Transportprozesse für Partikel in der PBL

\begin{tabular}{|l|l|l|}
\hline \multirow{2}{*}{ Luftschicht } & \multicolumn{2}{|c|}{ Hauptsächlich wirksamer Transport } \\
\cline { 2 - 3 } & Partikeldurchmesser $<0,1 \mu \mathrm{m}$ & \multicolumn{1}{|c|}{ Partikeldurchmesser $>1 \mu \mathrm{m}$} \\
\hline turbulente \\
Grenzschicht & $\bullet$ turbulente Diffusion & $\begin{array}{l}\bullet \text { turbulente Diffusion } \\
\bullet \text { Sedimentation }\end{array}$ \\
\hline viskose Grenzschicht & $\bullet$ molekulare Diffusion & $\begin{array}{l}\text { - Sedimentation } \\
\text { Impaktion }\end{array}$ \\
\hline
\end{tabular}

Im Gegensatz zu Gasmolekülen, für die die inneren und äußeren Oberflächen eines Bestandes als Akzeptorflächen fungieren, werden Partikel ausschließlich an die äußeren Oberflächen von Pflanzenteilen angelagert. 
Theoretische Überlegungen zum Stoffaustausch zwischen Atmosphäre und Vegetation

\section{a) Sedimentation ${ }^{7}$ im Schwerefeld der Erde}

Die Partikelablagerung in Folge der Schwerkraft der Erde ist der dominierende Senkenprozess für Riesenpartikel. Bei einem Durchmesser von 7,4 $\mu \mathrm{m}$ beträgt ihr Anteil mehr als $10 \%$, bei einem Durchmesser von $20 \mu \mathrm{m}$ mehr als $50 \%$ am Gesamtfluss (RUIJGROK ET AL., 1997). Für kleine Partikel spielt die Sedimentation eine vernachlässigbare Rolle, da diese auf Grund ihrer geringen Größe ein nahezu gleiches Diffusionsverhalten wie Luftmoleküle aufweisen und deshalb wie diese ständig mit den anderen Bestandteilen der Atmosphäre durchmischt werden.

Die Sedimentationsgeschwindigkeit $v_{g}$ luftgetragener Partikel kann durch den Fall einer Kugel in der Luft beschrieben werden (WARNECK, 2000):

$$
\begin{aligned}
v_{g}(r)= & \left(2 g \rho_{\text {Par }} / 9 \eta\right) r^{2} \approx\left(2,38 \cdot 10^{-4}\right) r^{2} \cdot m \cdot s^{-1} \\
\text { mit: } r & \text { Partikelradius }(\mu \mathrm{m}) \\
g & \text { Erdbeschleunigung (Normwert } \left.9,81 \mathrm{~m} \mathrm{~s}^{-2}\right) \\
\rho_{\text {Par }} & \text { Partikeldichte (hier angenommen mit } \left.2 \mathrm{~kg} / \mathrm{dm}^{3}\right) \\
\eta & \text { Viskosität von Luft }\left(1,8310^{-5} \mathrm{~kg} \mathrm{~m}^{-1} \mathrm{~s}^{-1}\right)
\end{aligned}
$$

Für die angenommene Partikeldichte von $2 \mathrm{~kg} / \mathrm{dm}^{3}$ ergeben sich hieraus für Partikel mit einem Durchmesser von 1 bzw. $10 \mu \mathrm{m}$ Sinkgeschwindigkeiten von 0,006 und 0,6 cm/s.

\section{b) Thermische und turbulente Diffusion}

Als thermische Diffusion (auch: "molekulare Diffusion", "Brownsche Molekularbewegung”) wird der physikalische Ausgleichsprozess bezeichnet, in dessen Verlauf Teilchen (Atome, Moleküle, Kolloidteilchen) infolge ihrer Wärmebewegung von Orten höherer Konzentration zu solchen niedriger Konzentration gelangen, so dass ein Dichte- und Konzentrationsausgleich erfolgt. Sie ist wirksam vor allem für Gasmoleküle und Aitkenkerne.

Der Betrag der thermischen Diffusion ist proportional zum Konzentrationsunterschied:

$$
\begin{gathered}
F_{i}=-D \cdot \frac{\partial c}{\partial x_{i}} \\
\text { mit: } F_{i} \text { Transportrate in Richtung } x_{i}, i=(1,2,3) \\
D \quad \text { Molekularer Diffusionskoeffizient }
\end{gathered}
$$

Die molekulare Diffusionskonstante $D$ hängt von der Partikelbeweglichkeit, der Lufttemperatur und - in geringem Maße - von der Partikelanzahl ab.

Turbulente Diffusion (auch: "turbulenter Transport") funktioniert analog zur thermischen Diffusion. Allerdings werden hier anstatt einzelner Moleküle ganze Luftelemente ("Luftpakete") mit jeweils unterschiedlichen Eigenschaften, wie z.B. unterschiedlichen Spurenstoffkonzentrationen ausgetauscht. Antrieb des turbulenten Transportes sind wie bei der thermi-

\footnotetext{
${ }^{7}$ Sedimentation (lat. sedimen): "Satz", "Bodensatz"
} 
Theoretische Überlegungen zum Stoffaustausch zwischen Atmosphäre und Vegetation

schen Diffusion Konzentrations- oder Temperaturdifferenzen. Zusätzlich wird aber die Existenz von eddies als Trägerelemente, also das Vorliegen einer turbulenten Strömung vorausgesetzt. In der Atmosphäre ist der turbulente Transport im allgemeinen um mehrere Größenordnungen effektiver als die thermische Diffusion, so dass die letztere bei Vorhandensein einer turbulenten Strömung ohne weiteres vernachlässigt werden kann (LILJEQUIST \& CEHAK, 1979).

\section{c) Impaktion}

Unter Impaktion versteht man die Trägheitsabscheidung von luftgetragenen Partikeln an Oberflächen. Diese tritt auf, wenn die Luftströmung in Folge eines Hindernisses umgelenkt wird, in der Luft enthaltene Teilchen jedoch dieser Umlenkung auf Grund ihrer Trägheit nicht folgen können. Im Gegensatz zur Diffusion werden bei der Impaktion vor allem Riesenpartikel $(>2 \mu \mathrm{m})$ deponiert, da die kleineren Partikel Richtungsänderungen der Luftströmung ausreichend rasch folgen können. Die Abscheidewahrscheinlichkeit wächst mit der Partikelgröße, der Anströmgeschwindigkeit und mit abnehmender Hindernisgröße.

\subsubsection{Quantifizierung der Trockenen Deposition luftgetragener Partikel}

Die Trockene Deposition luftgetragener Teilchen kann mit Hilfe der Partikelflussdichte bzw. der Depositionsgeschwindigkeit quantifiziert werden.

Die Partikelflussdichte $F_{\text {Par }}$ (auch: "Partikeltransferrate", "Partikeltransportrate" oder "Depositionsrate") beschreibt die Anzahl - alternativ die Gesamtmasse - der Partikel, die innerhalb einer Zeitspanne auf einer bestimmten Fläche deponiert werden. Zur Charakterisierung der Deposition auf natürlichen Vegetationsoberflächen nutzt man als Bezugsfläche in der Regel die vertikal projizierte, von der Vegetation bestandene Erdoberfläche (Grundfläche), nicht jedoch die Blattfläche eines Bestandes. In Analogie zum Begriff des latenten oder fühlbaren "Wärmeflusses" wird an Stelle des Begriffes "Partikelflussdichte" häufig der physikalisch weniger korrekte Begriff "Partikelfluss" verwendet, obwohl an sich eine Fluss- oder Stromdichte betrachtet wird.

Die Depositionsgeschwindigkeit $v_{d}$ von Partikeln ist die mit der Konzentration $c$ in der Referenzhöhe $z$ normierte Partikelflussdichte:

$$
v_{d}(z)=-F_{P a r} / c(z)
$$

In der allgemeineren Form wird der von CHAMBERLAIN (1960) eingeführte Begriff der Depositionsgeschwindigkeit als Quotient der Depositionsrate und der Differenz $\Delta c$ der Spurenstoffkonzentration zwischen Referenzhöhe und Akzeptorfläche definiert. Im Falle der Partikeldeposition wird jedoch angenommen, dass die Partikelkonzentration an der Akzeptorfläche Null sei, so dass gilt:

$$
\Delta c=c(z)
$$


Theoretische Überlegungen zum Stoffaustausch zwischen Atmosphäre und Vegetation

Damit kann die vereinfachte Definition gewählt werden. Das Minuszeichen in (6) ergibt sich aus der Konvention, dass ein positiver Wert für $F_{P a r}$ üblicher Weise einen Fluss vom Erdboden in die Atmosphäre darstellt.

Anschaulich kann man sich die Depositionsgeschwindigkeit vorstellen als die mittlere Geschwindigkeit, mit der sich Spurengasmoleküle oder Partikel auf eine Senke zu bewegen. Für große Partikel (Durchmesser $>10 \mu \mathrm{m}$ ) nähert sich die Depositionsgeschwindigkeit der Sinkgeschwindigkeit an und erhält so eine noch anschaulichere Bedeutung.

Die Depositionsgeschwindigkeit ist ein Maß für die Effizienz des Transports über einer Oberfläche, das die Leitfähigkeit des Systems Atmosphäre - Akzeptoroberfläche für den Spurenstofftransport angibt. Der Betrag hängt vom betrachteten Spurenstoff, den Eigenschaften der Depositionsflächen und den Austauschverhältnissen ab. Die Depositionsgeschwindigkeit ermöglicht einen Vergleich des Ablagerungsverhaltens unterschiedlicher Spurenstoffe und Oberflächen bei bestimmten mikrometeorologischen Bedingungen, unabhängig von den herrschenden Luftkonzentrationen. Ist die Depositionsgeschwindigkeit eines Stoffes bekannt oder kann diese in befriedigender Weise modelliert werden, kann die Deposition eines Spurenelements bereits aus Konzentrationsmessungen dieses Stoffes ermittelt werden ("Konzentrationsmethode", z.B. MicHAELIS ET AL., 1992).

In Analogie zur elektrischen Leitfähigkeit kann die Depositionsgeschwindigkeit als Kehrwert eines Transportwiderstandes angesehen werden. Es ist üblich, diesen Widerstand nach der Beziehung

$$
v_{d}^{-1}=r_{a}+r_{b}+r_{c}
$$

in drei hintereinander geschachtelte Einzelwiderstände zu untergliedern, wobei mit $r_{a}$ der aerodynamische Widerstand, mit $r_{b}$ der Widerstand bei der Überwindung der oberflächennahen laminaren Grenzschicht und mit $r_{c}$ der Oberflächenwiderstand bezeichnet werden ("Widerstandsmodell", vgl. hierzu z.B. MEYERS \& BALDOCCHI, 1988; CONSTANTIN, 1993; GRAVENHORST ET AL., 2000).

Wenn ein Spurenstoff von der Vegetation netto nicht deponiert, sondern emittiert wird, ergeben sich negative Depositionsgeschwindigkeiten. Im Falle von Partikeln ist neben der direkten Freisetzung von organischem Material, wie Samen und Pollen, z.B. die zeitweilige Emission von Terpenen möglich, aus denen bei bestimmten Reaktionsbedingungen in der Atmosphäre neue Partikel innerhalb oder kurz oberhalb des Vegetationskörpers gebildet und in die Atmosphäre hinein transportiert werden. Da in diesen Fällen die Bezeichnung Depositionsgeschwindigkeit nicht mehr sinnvoll anwendbar ist, sollte hier der allgemeinere Begriff der vertikalen Transportgeschwindigkeit Verwendung finden (z.B. STULL, 1988).

\subsubsection{Methoden der experimentellen Bestimmung der Trockenen Deposition luftgetragener Partikel}

Die Trockene Deposition luftgetragener Partikel kann mit Hilfe verschiedener direkter und 
Theoretische Überlegungen zum Stoffaustausch zwischen Atmosphäre und Vegetation

indirekter Methoden experimentell quantifiziert werden (Tabelle 3).

Tabelle 3: Ausgewählte Methoden zur Bestimmung der Trockenen Deposition luftgetragener Partikel

\begin{tabular}{|c|c|c|}
\hline \multirow[t]{2}{*}{ Direkte Methoden } & \multicolumn{2}{|c|}{ Indirekte Methoden } \\
\hline & $\begin{array}{l}\text { Ökosystemare Methoden } \\
\text { (Massenbilanz-Methoden) }\end{array}$ & $\begin{array}{c}\text { Mikrometeorologische } \\
\text { Methoden }\end{array}$ \\
\hline $\begin{array}{l}\text { - künstliche Behälter } \\
\text { - Elektronenmikroskop } \\
\text { - Abwaschverfahren } \\
\text { - Radioaktivitätsmessungen }\end{array}$ & - Kronentraufendifferenz & $\begin{array}{l}\text { - Gradientmethoden } \\
\text { - Eddy-Kovarianzmethode } \\
\text { - Akkumulationsmethode }\end{array}$ \\
\hline
\end{tabular}

\section{a) Direkte Methoden zur Bestimmung der Trockenen Deposition luftgetragener Partikel}

Die direkten Methoden zur Bestimmung der Trockenen Deposition von Partikeln sind dadurch gekennzeichnet, dass die zeitliche Änderung der Anzahldichte Partikel auf Pflanzenteilen oder auf Stellvertreterflächen direkt untersucht wird. Hierfür werden entweder Probe-Pflanzen mit einer wohl definierten Partikelbelegung oder künstliche Oberflächen (Surrogatflächen) jeweils für mehrere Stunden oder Tage bei Abwesenheit von Niederschlag in der Atmosphäre exponiert und später analysiert.

Die Verwendung von künstlichen Sammelbehältern und Pflanzennachbildungen ist generell kritisch, da die natürlichen Oberflächen der realen Vegetation mit künstlichen Oberflächen kaum nachzubilden sind (FAIRALL, 1984; GRAVENHORST \& WARAGHAI, 1990). Verwendung finden entweder Trichter oder Töpfe als Sedimentationsgefäße (z.B. DÄMMGEN ET AL., 1990), die teilweise bereits während der Expositionszeit mit Natronlauge, Salzsäure oder ähnlichen Flüssigkeiten gefüllt sind, oder Pflanzennachbildungen aus Kunststoff (z.B. SCHLEGEL, 1989; HOFSCHREUDER ET AL., 1996). Da die Oberflächeneigenschaften der Surrogatflächen nicht denjenigen der jeweiligen Pflanzendecke entsprechen, kommt es zu einer Verfälschung der Ergebnisse insbesondere für Stoffgruppen, die nicht vorwiegend sedimentierend deponiert werden. Deshalb stellen die so ermittelten Beträge nur potentielle Depositionen in Hinblick auf einen Pflanzenbestand oder einen Boden dar (DÄMMGEN ET AL., 1990).

Bei der Nutzung von echten Vegetationselementen als Akzeptorflächen ergibt sich die Notwendigkeit, die Probepflanzen vor ihrer Exponierung in der Atmosphäre ausreichend zu reinigen. Hierbei müssen mindestens so viele Partikel von der Oberfläche entfernt werden, dass nach der Exponierung der Pflanzen signifikante Unterschiede in der Partikelbelegung zwischen exponierten und nicht exponierten Pflanzen aufgezeigt werden können. Die Anforderungen an den Grad der Reinheit werden deshalb von dem zur Anwendung kommenden Analyseverfahren bestimmt. Ob eine vollständige Entfernung der Partikel von der Pflanzenoberfläche möglich ist, ist umstritten. Eigene Untersuchungen im Zuge der 
Theoretische Überlegungen zum Stoffaustausch zwischen Atmosphäre und Vegetation

vorliegenden Arbeit deuten darauf hin, dass dies für Fichtennadeln mit herkömmlichen Methoden (Wasser, Druckluft, Ultraschall, Alkohol) kaum befriedigend möglich ist (vgl. Abb. 2). Ursächlich könnten diese Schwierigkeiten mit der Tatsache zusammenhängen, dass Partikel auf Fichtennadeln teilweise in der oberflächlichen Wachsschicht des Nadelblattes fixiert werden. Eine vollständige Reinigung der Nadel würde deshalb die Entfernung dieser Wachsschicht voraussetzen. Dies ist theoretisch möglich, würde aber letztendlich zu einer unakzeptablen Verfälschung der Oberflächeneigenschaften der Nadel führen.
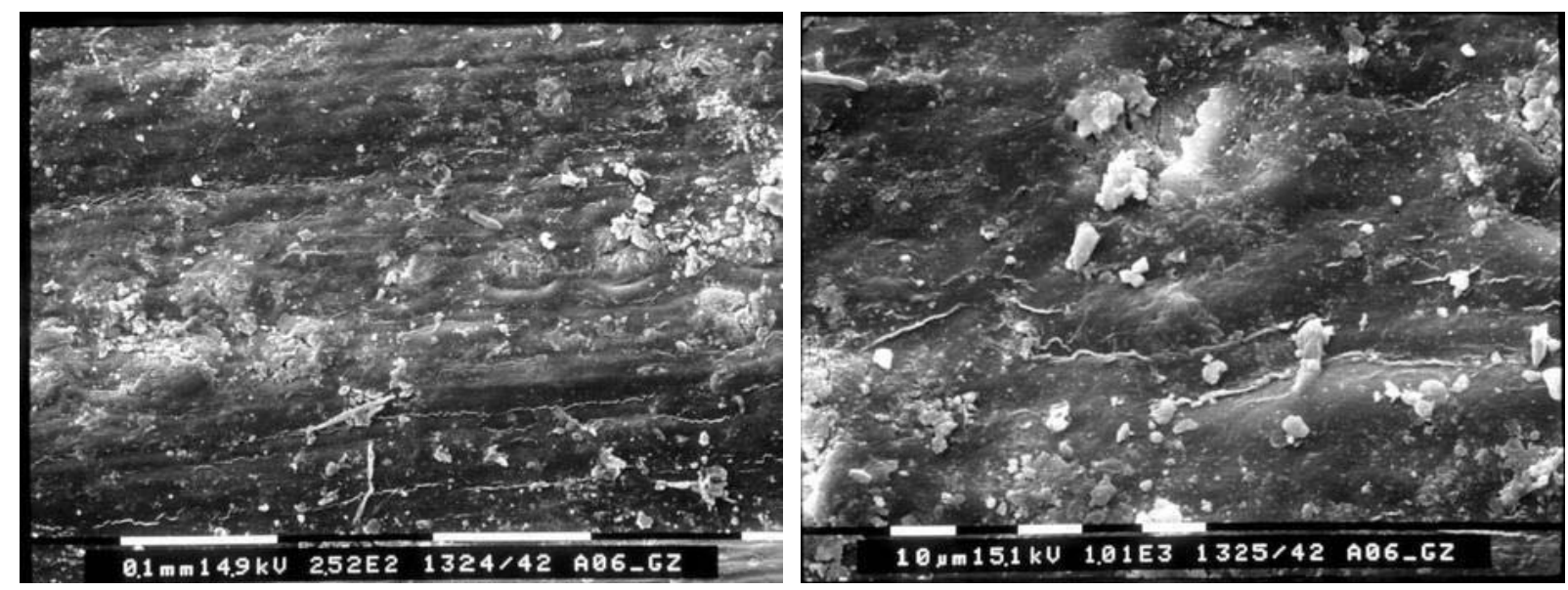

Abb. 2: Fichtennadeln unter dem Elektronenmikroskop (eigene Aufnahmen): Ausschnitte einer mit Ultraschall gereinigten Nadeloberfläche. Ein weißer Balken entspricht einer Strecke von 0,1 mm (links) bzw. 0,01 mm (rechts).

Alternativ können Pflanzen unter Reinluft aufgezogen und einmalig in der Atmosphäre exponiert werden. Auf diese Art und Weise werden allerdings fast ausschließlich sehr junge Pflanzen oder Pflanzenteile exponiert, deren Oberflächeneigenschaften wiederum nicht identisch mit denjenigen älterer Pflanzen sind.

Für die Analyse der Akzeptorflächen werden diese abgewaschen; das Abwaschwasser wird anschließend im Labor qualitativ und quantitativ analysiert (“Abwaschverfahren”, z.B. MARQUeS, 1998; HofschreUder ET AL., 1996). Bei diesem Verfahren kann die Partikeldeposition jedoch nicht unabhängig von der Gasdeposition bestimmt werden. Alternativ können die Flächen unter einem Elektronenmikroskop betrachtet und dort die deponierten Partikel in ausgewählten Bereichen ausgezählt werden (z.B. GRAVENHORST \& WARAGHAI, 1990). Eine solche Analyse ist nur für geeignete Akzeptorflächen möglich, auf denen die deponierten Teilchen deutlich identifiziert werden können. Bei sehr jungen, unter Reinluft aufgezogenen Fichten ist die Nadeloberfläche beispielsweise mit einer stäbchenförmigen Wachsstruktur überzogen, die das Auszählen von deponierten Partikeln erschwert (Abb. 3; vgl. auch die Aufnahmen in SCHELL \& KRISTEN, 1992). Generell müssen bei der Verwendung des Elektronenmikroskops enorme statische Unsicherheiten in Kauf genommen werden, da jeweils nur sehr kleine Ausschnitte $\left(<0,01 \mathrm{~mm}^{2}\right)$ der Gesamtfläche 
Theoretische Überlegungen zum Stoffaustausch zwischen Atmosphäre und Vegetation

betrachtet werden können. Zudem werden die Partikel unter dem Mikroskop in Abwesenheit von Wasser untersucht, so dass eine auf diese Art und Weise bestimmte Partikelgröße unter Umständen nicht mit der Größe des ursprünglichen, luftgetragenen Partikels übereinstimmt.
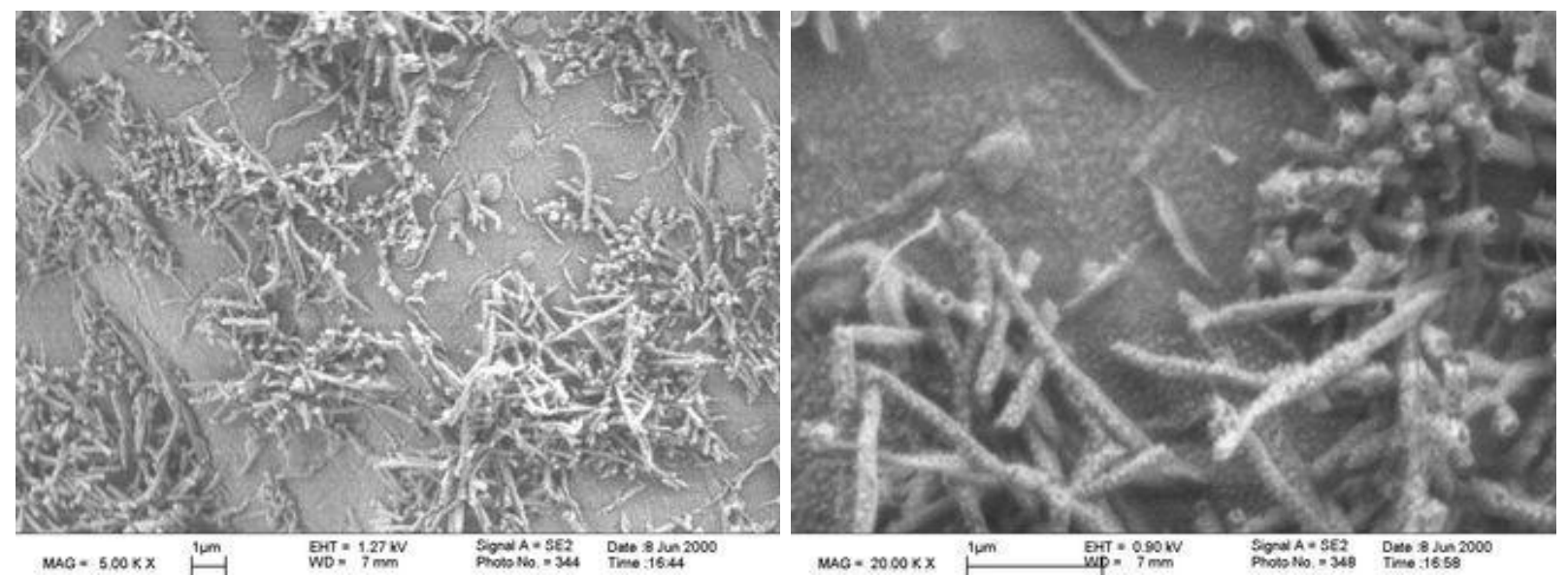

Abb. 3: Fichtennadeln unter dem Elektronenmikroskop (eigene Aufnahmen): Ausschnitte der Oberfläche einer unter Reinluft ausgetriebenen Nadel mit stäbchenförmiger Wachsstruktur

Eine Sonderstellung nehmen Untersuchungen mit kurzlebigen, radioaktiven Isotopen wie ${ }^{214} \mathrm{~Pb}$ ein, die in der Atmosphäre gebildet werden und sich an luftgetragene Partikel anheften. Hierbei werden Pflanzenelemente, die für einige Zeit in geschlossenen Räumen aufbewahrt wurden, für mehrere Stunden in der Atmosphäre exponiert und anschließend auf radioaktive Strahlung untersucht (z.B. Wyers \& VeltKAMP, 1996). Es wird angenommen, dass die gemessene radioaktive Strahlung proportional zur Menge der deponierten Partikel ist. Die Zuordnung der ermittelten Depositionswerte zur Partikelgröße der deponierten Teilchen ist allerdings unter Umständen schwierig. Auch stellen die niedrigen Konzentrationen der natürlich gebildeten Isotope hohe Anforderungen an die Messgenauigkeit der verwendeten Apparaturen (BUTTERWECK, 1991). Dieses Problem lässt sich mit dem Einsatz künstlich radioaktiv markierter Partikel umgehen (z.B. JONAS, 1994), jedoch ist deren Verwendung aus Gründen des Strahlungsschutzes nicht überall über längere Zeiträume möglich.

Für die Analyse bestimmter chemischer Inhaltstoffe im abgelagerten Partikelmaterial kann auch das Filmabziehverfahren die Methode der Wahl sein. Hier werden Blätter mit gelösten Kunststoff bestrichen. Nach dem Trocknen wird der Film mit den anhaftenden Partikeln abgezogen. Für Nadelbäume ist die Methode allerdings ungeeignet (CERCACOV, 1985).

Bei den vorgestellten direkten Untersuchungsmethoden handelt es sich immer um punktuelle Messungen, aus denen flächenhafte Aussagen nur bei genauer Kenntnis der Biomasseverteilung und deshalb in der Regel nur mit beträchtlichen Unsicherheiten abgeleitet werden können. So finden beispielsweise DÄMMGEN ET AL. (1990) bei der Bestimmung von Stickstoff- und Schwefeleinträgen mit Sammelbehältern große Streuungen bei der Auswertung paralleler Messungen und nicht zuordenbare Ausreißer. Weiterhin können die Beobachtungen insbesondere beim Abwaschverfahren durch die Adsorption von 
Theoretische Überlegungen zum Stoffaustausch zwischen Atmosphäre und Vegetation

Gasmolekülen an den Akzeptorflächen verfälscht werden. Denkbar sind auch Veränderungen der deponierten Spezies im Zeitfenster zwischen Deposition und Analyse, beispielsweise durch einen mikrobiellen Abbau organischer Partikel.

\section{b) Ökosystemare Methoden zur Bestimmung der Trockenen Deposition luftgetragener Partikel: die Interpretation der Kronentraufe}

Für die Interpretation der Kronentraufe (z.B. IBROM, 1993) wird der Bestandsniederschlag eines Waldes (die "Kronentraufe") in bulk Sammlern aufgefangen und chemisch analysiert. Ausgehend von der Annahme, dass der Niederschlag die auf den Blättern trocken deponierten Stoffe vollständig abwäscht, enthält die Kronentraufe den gesamten atmosphärischen Eintrag von (löslichen) Spurenstoffen aus Nasser und Trockener Deposition. Ist aus zusätzlichen, unabhängigen Messungen der Stofffluss im Regen über dem Kronenraum bekannt, kann nach Constantin (1993) die Rate der Trockenen Deposition aus der Differenz der beiden Stoffflüsse und unter Berücksichtigung der Kronen-Regen-Interaktion (KRI) interpretiert werden aus:

$$
\begin{array}{cl}
\qquad B N=F N+F_{T D}+K R I \\
\text { mit: } B N \quad \text { Stofffluss unter dem Kronendach (Bestandsniederschlag) } \\
F N \quad \text { Stofffluss über dem Kronendach (Freilandniederschlag) } \\
F_{T D} \text { Trockene Deposition (Gase und Partikel) } \\
\text { KRI } & \text { Kronen-Regen-Interaktion }
\end{array}
$$

$F_{T D}$ muss um den Betrag der Gasdeposition korrigiert werden, um auf die Partikelflussdichte schließen zu können.

Der (elementspezifische) Betrag der KRI hängt von der Pflanzenart, dem Konzentrationsgefälle zwischen Zellsaftlösung und Außenkonzentration sowie einer Reihe physiologischer Größen ab (IBROM, 1993). Dabei ist es sowohl möglich, dass die trocken deponierten Stoffe bereits vor einem folgenden Niederschlag über die Blätter einer Pflanze aufgenommen werden und deshalb in - relativ zu der realen Depositionsmenge - zu geringen Mengen im Bestandsniederschlag auftauchen, als auch, dass Elemente aus Pflanzenblättern ausgewaschen werden ("leaching") und deshalb in $\mathrm{zu}$ hohen Konzentrationen im Bestandsniederschlag bestimmt werden. Die Interpretation der Kronentraufe ist deshalb grundsätzlich nur für solche Elemente geeignet, für die die Kronen-Regen-Interaktionen genau quantifizierbar und/oder vernachlässigbar klein sind.

Für einige Baumarten, wie z.B. Buchen, ist es notwendig, zusätzlich zum Bestandsniederschlag den Stammablauf zu sammeln. Für eine vollständige Erfassung des Eintrags unlöslicher oder nur teilweise löslicher Spurenstoffe, wie z.B. Schwermetallen, muss neben der Kronentraufe auch der Streufall analysiert werden, da diese Stoffe teilweise auf den Blättern haften bleiben und erst mit dem Blattfall den Boden erreichen (CONSTANTIN, 1993; MEESENBURG ET AL., 1997). 
Theoretische Überlegungen zum Stoffaustausch zwischen Atmosphäre und Vegetation

Die Interpretation der Kronentraufe eignet sich gut für Stoffbilanzierungen von Waldökosystemen über längere Zeiträume. Da - je nach Niederschlagsmenge - mit einem Niederschlagsereignis immer nur ein Teil der trocken deponierten Stoffe abgewaschen werden, sind jedoch bereits Messungen über einen Zeitraum von weniger als einem Monat in ihren Resultaten sehr unzuverlässig (CONSTANTIN, 1993). Zeitlich hoch aufgelöste Prozesse lassen sich mit der Methode gar nicht untersuchen. In gleicher Weise wie die direkten Methoden handelt es sich bei der Interpretation der Kronentraufe um eine Punktmessung, aus welcher flächenhafte Aussagen nur mit einer Vielzahl von Parallelmessungen abgeleitet werden können.

\section{c) Mikrometeorologische Methoden zur Bestimmung der Trockenen Deposition}

Mit mikrometeorologischen Ansätzen wird der Betrag der Trockenen Deposition von Partikeln indirekt bestimmt, indem der vertikale Partikeltransport durch die atmosphärische Grenzschicht quantifiziert und über eine geeignete Mittelungszeit integriert wird. Der totale vertikale Stofffluss an Partikeln zwischen Atmosphäre und Erdoberfläche (nach TwOREK, 1996, verändert):

$$
\begin{aligned}
& \qquad \vec{F}_{\text {Par }}=\frac{1}{\tau} \int_{0}^{\tau} \vec{w} c d t \\
& \text { mit: } F_{\text {Par }} \text { vertikaler Partikelfluss }\left(\mathrm{m}^{-2} \mathrm{~s}^{-1}\right) \\
& \tau \text { Mittelungszeit }(\mathrm{s}) \\
& w \text { vertikale Komponente des Windvektors }(\mathrm{m} / \mathrm{s}) \\
& c \text { Partikelanzahldichte }\left(\mathrm{m}^{-3}\right)
\end{aligned}
$$

wird dabei als Äquivalent zu einem Stoffein- bzw. Stoffaustrag in bzw. aus einem Pflanzenbestand interpretiert. Dies setzt voraus, dass der vertikale Partikelfluss mit dem Gesamtpartikelfluss identisch ist, also die Summe der horizontalen Flüsse gleich Null ist.

Weiter wird davon ausgegangen, dass der betrachtete vertikale Fluss turbulenter Natur und dem Konzentrationsgradienten entgegengesetzt gerichtet ist und nicht mit der Höhe variiert. Es wird angenommen, dass keine unbekannten Quellen oder Senken für Partikel existieren und diese auf ihren Weg zwischen Atmosphäre und Vegetation nicht chemisch verändert werden.

Unter diesen Voraussetzungen ist es möglich, mit verschiedenen, in der Mikrometeorologie für die Bestimmung von Stoff- und Energieflüssen wohl bekannten Methoden den vertikalen Partikelfluss zu quantifizieren. Hierzu gehört der Gradientansatz (vgl. MONIN \& OBUKHOV, 1954), die Eddy - Kovarianzmethode (vgl. BALDOCCHI ET AL., 1988) sowie die Eddy Akkumulationsmethode. Die einzelnen Ansätze werden im Kapitel 2 näher vorgestellt.

Alle Methoden zeichnen sich dadurch aus, dass kontinuierliche, flächenhafte und zeitlich hoch aufgelöste Messungen möglich sind. Der Einsatz mikrometeorologischer Methoden zur Bestimmung des Partikelflusses ist gleichzeitig aber auch noch mit beträchtlichen Unsicherheiten verbunden (BESWICK ET AL., 1994). Insbesondere stimmen Ergebnisse von 
Theoretische Überlegungen zum Stoffaustausch zwischen Atmosphäre und Vegetation

Messungen mit unterschiedlichen mikrometeorologischen Methoden an ein und demselben Ort und zu ein und derselben Zeit durchaus nicht immer überein (RUIJGROK ET AL., 1997).

Gegenüber den anderen vorgestellten Methoden unterscheiden sich mikrometeorologische Methoden dadurch, dass mögliche Emissionsflüsse von Partikeln aus der Vegetation in die Atmosphäre miterfasst werden, so dass die Ergebnisse unter Umständen nicht direkt mit den Ergebnissen der anderen Methoden verglichen werden können. Auch sind die ermittelten Depositionsgeschwindigkeiten $v_{d}$ lokale Geschwindigkeiten in der Referenzhöhe $z$, die mit der Deposition auf der Erdoberfläche $v_{d s}$ selbst in folgender Beziehung stehen:

$$
\begin{array}{cl}
\qquad v_{d s}=\left(v_{d}{ }^{-1}-r_{a}\right)^{-1} \\
\text { mit: } v_{d s} \text { Depositionsgeschwindigkeit an der Erdoberfläche } \\
r_{a} \text { aerodynamischer Widerstand zwischen Messhöhe } z \text { und Erdoberfläche }
\end{array}
$$

Der Betrag des aerodynamischen Widerstandes $r_{a}$ kann in erster Näherung aus $u / u_{*}{ }^{2}$ ermittelt werden. Da $u *$ jedoch in der Regel nur den mechanisch induzierten Teil der Gesamtturbulenz präsentiert (siehe Abschnitt 1.1.2), gilt dies nur, wenn der turbulente Transport durch die kleineren, durch die Windscherung produzierten Eddies dominiert wird, wie dies in einer gut ausgebildeten Prandtl-Schicht der Fall ist. Wird die Turbulenz dagegen durch große, kohärente, thermisch induzierte Strukturen charakterisiert, kann die Ähnlichkeitstheorie (MONIN \& OBUKHOV, 1954) nicht mehr angewendet werden, so dass der Wert für $r_{a}$ um einen stabilitätsabhängigen Betrag korrigiert werden muss.

Für Waldoberflächen liegt der Betrag des aerodynamische Widerstandes $r_{a}$ bei ca. $10 \mathrm{~s} / \mathrm{m}$, so dass der Unterschied zwischen $v_{d}$ und $v_{d s}$ in der Regel vernachlässigbar klein ist.

\subsubsection{Trockene Deposition und Depositionsgeschwindigkeit luftgetragener Partikel über Wäldern}

Für Wälder und Forste ist der Stoffeintrag aus der Atmosphäre von ganz besonderer Bedeutung, da diese Ökosysteme im Gegensatz zu landwirtschaftlichen Nutzflächen in der Regel nicht gedüngt werden, und der atmosphärische Stoffeintrag somit faktisch die einzige Quelle von Schad- und Nährstoffen für das System ist. Für die Vitalität der Waldbestände hat dabei der atmosphärische Eintrag von Säuren, die einen Abbau der basischen Nährstoffvorräte im Boden bewirken und damit einen Nährstoffmangel der Bäume verursachen, sowie der Eintrag von Nährstoffen und toxischen Schwermetallen eine besondere Bedeutung. Für einige chemische Elemente wie Magnesium oder Calcium ist der atmosphärische Nährstoffeintrag für die existierenden Wälder bereits essentiell, da die Vorräte im Boden inzwischen sehr stark reduziert sind, und die Bäume ihren Bedarf mehr oder weniger vollständig aus den atmosphärischen Eintrag decken müssen. Bei anderen Stoffen wie Stickstoff oder Schwefel übersteigt der Eintrag aus der Luft offensichtlich bei weitem den Bedarf, so dass mit einer zunehmenden Eutrophierung der Wälder durch Stickstoff zu rechnen ist (MATZNER \& MurACH, 1995; MEESENBURG ET AL., 1997; IBROM ET AL., 1995). 
Theoretische Überlegungen zum Stoffaustausch zwischen Atmosphäre und Vegetation

Über die Größenordnung der Trockenen Deposition über Wäldern existieren bis jetzt nur wenige, aus punktuellen Messungen gewonnene Schätzwerte. Allgemein anerkannt ist, dass Waldbestände auf Grund ihrer hohen Rauhigkeit und des großen Blattflächenindexes im Vergleich zu anderen Vegetationsformen sehr effektive Senken für luftgetragene Partikel und Gase sind (z.B. UlRICH ET AL., 1979; WIMAN ET AL., 1990). Für einige Forststandorte wird die Gesamtdeposition (Nasse und Trockene Deposition) für bestimmte chemische Elemente, wie Schwefel, Arsen, Cadmium und Blei, sogar von der trockenen Ablagerung dominiert (BergKVist, 1987; Michaelis ET AL., 1992). Dabei ist die Rate der Trockenen Deposition baumartenspezifisch, so dass in Nadelwäldern generell größere Stoffmengen trocken deponiert werden als in Laubwäldern (HÖFKEN \& GRAVENHORST, 1983).

Die exakte Höhe der Depositionsgeschwindigkeit über Wäldern ist nicht sicher bekannt. Die von verschiedenen Autoren (z.B. HICKS ET AL., 1982; GravenHORST \& WARAGHAI, 1990; ERISMAN ET AL., 1997a; BUZORIUS ET AL., 1998) unter unterschiedlichen meteorologischen und standörtlichen Randbedingungen ermittelten mittleren Depositionsgeschwindigkeiten für Partikel über Wäldern liegen um etwa einer Größenordnung über denjenigen von Wasser- und Grasflächen (z.B. WeSELY ET AL., 1985; DUAN ET AL., 1988 u.a.). Die Resultate von Messungen zeigen untereinander große Variabilitäten insbesondere bezüglich der Absolutwerte der Depositionsgeschwindigkeit für submikroskopisch kleine Partikel, zu dessen Depositionsgeschwindigkeit heute nur sehr unsichere Aussagen gemacht werden können (GALLAGHER ET AL., 1997). Nicht immer stimmen die Ergebnisse von Messungen mit theoretischen Berechnungen überein (z.B. RUIJGROK ET AL., 1997).

Es besteht Einigkeit darüber, dass die Depositionsgeschwindigkeiten luftgetragener Partikel mit höheren Windgeschwindigkeiten (GRAVENHORST \& WARAGHAI, 1990) bzw. höheren Schubspannungsgeschwindigkeiten (ERISMAN ET AL., 1997a) prinzipiell zunehmen. Weiter ist bekannt, dass die Depositionsgeschwindigkeit von der Größe der Partikel abhängt (z.B. LORENZ \& MURPHY, 1989), wie dies bereits auch aus Windkanaluntersuchungen vorhergesagt worden ist (SEHMEL \& HodgSON, 1980). Genauere Kenntnisse über das Verhalten kleinerer Partikel über Wäldern fehlen jedoch. So ermitteln GALLAGHER ET AL. (1997) eine Zunahme der Depositionsgeschwindigkeit mit der Größe der Partikel im Messbereich zwischen 0,1 und $1 \mu \mathrm{m}$ Durchmesser, während andere Autoren (z.B. Constantin, 1993) für denselben Größenbereich eine Abnahme der Depositionsgeschwindigkeit mit der Größe annehmen. Einigkeit herrscht darüber, dass der Grad der Abhängigkeit der Depositionsgeschwindigkeit von der Windgeschwindigkeit (Schubspannungsgeschwindigkeit) selbst wiederum von der Partikelgröße abhängt: die Deposition größerer Partikel wird mit zunehmendem Wind stärker forciert als diejenige kleinerer Partikel (GRAVENHORST \& WARAGHAI, 1990; RUIJGROK ET AL., 1997). 


\section{Mikrometeorologische Methoden zur Bestimmung vertikaler Partikelflussdichten unter besonderer Berücksichtigung der Eddy - Kovarianzmethode}

In diesem Kapitel werden die wichtigsten mikrometeorologischen Methoden zur experimentellen Bestimmung vertikaler, turbulenter Stoff- und Energieflüsse zwischen Atmosphäre und Erdoberfläche vorgestellt und ihre Eignung für die Erfassung von Partikelflussdichten diskutiert. Auf die Eddy - Kovarianzmethode wird besonders detailliert eingegangen, da diese Methode der vorliegenden Arbeit zu Grunde liegt. Übliche Korrekturen der Kovarianzmethode werden beschrieben und die Notwendigkeit des Einsatzes dieser Korrekturen bei der Bestimmung von Partikelflussdichten untersucht.

\subsection{Gradientmethoden}

Gradientmethoden sind - im Gegensatz zu der später beschriebenen Eddy Kovarianzmethode sowie der Eddy - Akkumulationsmethode - indirekte Methoden für die Bestimmung vertikaler Stoff- und Energieflüsse. Sie basieren auf den theoretischen Arbeiten von Monin \& OBuKHOV (1954) und setzen die Kenntnis des Mischungsverhältnisses eines Spurenstoffes in mindestens zwei Höhen voraus. Aus der über einer ausreichend langen Zeitspanne $(\approx 30$ Minuten) gemittelten Konzentrationsdifferenz wird unter Berücksichtigung eines turbulenten Diffusionskoeffizienten eine vertikale Stoffflussdichte $F$ modelliert:

$$
F=K_{e} \frac{\partial c}{\partial z}
$$

mit: $K_{e}$ turbulenter Diffusionskoeffizient

$\frac{\partial c}{\partial z} \quad$ vertikale Konzentrationsdifferenz

Dabei wird angenommen, dass der Betrag des Diffusionskoeffizienten nur von der atmosphärischen Turbulenz, nicht aber vom betrachteten Spurenstoff abhängig ist.

Für die notwendige Abschätzung des Koeffizienten gibt es verschiedene Möglichkeiten. Bei der aerodynamischen Methode wird er aus Windprofilmessungen abgeleitet. Da der Koeffizient mit der Stabilität der Atmosphäre variiert, ist eine entsprechende Parametrisierung notwendig. Hieraus ergeben sich Unsicherheiten bezüglich der Ergebnisse der Methode, wobei bei niedrigen Windgeschwindigkeiten besonders große Fehler auftreten (BALDOCCHI ET AL., 1988). Für die Bowen-Verhältnis-Methode (Bowen ratio-energy balance method) wird der Koeffizient dagegen unter Benutzung der Energiebilanzgleichung (3) aus dem Verhältnis des Temperatur- und Feuchtegradienten indirekt über den fühlbaren und latenten Wärmestrom 
bestimmt. Probleme ergeben sich hier vor allem bei niedrigen Beträgen der Strahlungsbilanz also bei Sonnenauf- und Sonnenuntergang (STULL, 1988) bzw. generell während der Nacht, während des Winters oder bei starker Bewölkung (MAITANI, 1993). Auch muss berücksichtigt werden, dass die Energiebilanz mit Hilfe meteorologischer Messungen häufig nicht geschlossen werden kann, wie dies bereits in Kapitel 1.3.1 diskutiert wurde.

Gradientmethoden liefern gute Ergebnisse für Messungen über kurzer Vegetation. Problematisch ist ihre Anwendung jedoch innerhalb von Pflanzenbeständen (CELLIER \& BRUNET, 1992) und über Wäldern (HARTOG \& NEUMANN, 1984), da in diesen Fällen die Fluss-Gradienten-Beziehungen nicht mehr eindeutig definiert sind und der Durchzug von größeren Luftwirbeln am Messprofil vorbei zu Fehlabschätzungen des vertikalen Stoffflusses führen können (BALDOCCHI ET AL., 1988). Auch sind die vertikalen Konzentrationsdifferenzen in der sehr labilen Prandtl-Schicht über der rauhen Oberfläche eines Waldes besonders klein (BUZORIUS ET AL., 1998; HERTLEIN, 1990), so dass für ihre Auflösung sehr genaue Messinstrumente benötigt werden.

Die praktische Umsetzung der Gradientmethode für die Bestimmung des Partikelflusses mit Hilfe optischer Analysatoren wird dadurch erschwert, dass für diesen Zweck der parallele Einsatz zweier Geräte notwendig ist. Der Grund hierfür ist die Tatsache, dass das Ansaugen der Probeluft zum Analysator, in dem die Partikelkonzentration bestimmt wird, nur über eine sehr kurze Entfernung von einigen wenigen Metern möglich ist, da andernfalls - im Gegensatz zu Konzentrationsmessungen inerter Spurengase - zu große Konzentrationsverluste im Ansaugrohr zu erwarten sind.

Ein Beispiel für eine Anwendung der Gradientmethode für die Bestimmung des Partikelflusses unter Benutzung zweier 5-stufiger Schlitzdüsenimpaktoren gibt HERTLEIN (1990). Die zeitliche Auflösung seiner Ergebnisse ist mit durchschnittlich 48 Stunden allerdings sehr gering. Nur in diesen langen Zeitspannen konnten die geringen Konzentrationsunterschiede in ausreichendem Maße aufgelöst werden. Eine Anwendung der Gradientmethode unter Verwendung von Filtern beschreiben WYERS \& DUYZER (1997), die sich allerdings auf Sulfat- und Nitratpartikel beschränken und eine zeitliche Auflösung von zwei Stunden realisieren.

\subsection{Die Eddy - Kovarianzmethode}

Seit den 50er Jahren wird zur Bestimmung turbulenter Flüsse in der Planetarischen Grenzschicht der Atmosphäre das Prinzip der Eddy - Kovarianz angewandt (MAITANI, 1993). Auch wenn es zunächst eine gewisse Zeit brauchte, bis - in den 60er resp. 70er Jahren - erste feldtaugliche Ultraschall-Anemometer auf den Markt kamen, ist die Eddy-Kovarianzmethode heute eine anerkannte und weitverbreitete Methode in der Mikrometeorologie. Auf dem einfachen theoretischen Konzept der Korrelation von Vertikalwind und Temperatur, Wasserdampf oder Stoffkonzentration beruhend, hat sie sich als direkte Möglichkeit erwiesen, die Strukturen der turbulenten Strömung in der bodennahen Atmosphäre aufzulösen und die 
mit ihnen verbundenen Stoff- und Energieflüsse direkt zu quantifizieren (GRAVENHORST ET AL., 2000). Für ihre Anwendung bestehen nur wenige Einschränkungen bezüglich Oberflächenbeschaffenheit oder Atmosphäre (ARYA, 1988), sie ist zudem eine der wenigen Möglichkeiten, turbulente Flüsse auch innerhalb von Pflanzenbeständen zu messen (LEUNING \& MONCRIEFF, 1990). Als Nachteil der Methode wird die Notwendigkeit des Einsatzes sehr schneller und damit auch relativ teurer Messgeräte genannt, die zudem heute nur für wenige Spurenstoffe zu Verfügung stehen (STULL, 1988). Als problematisch wird auch der notwendige hohe Aufwand für die Speicherung und Auswertung großer Datenmengen empfunden (UNLAND ET AL., 1996) - ein Einwand, der heute freilich durch die Entwicklung einer leistungsfähigen Computerhard- und -software zunehmend an Bedeutung verliert.

\subsubsection{Beschreibung der Methode}

Ausgangspunkt der Eddy-Kovarianzmethode ist zunächst die hochfrequente $(\approx 10 \mathrm{~Hz})$ Erfassung der Zeitreihen des dreidimensionalen Windfeldes, der Temperatur, der Feuchte und - je nach Interesse - der Konzentrationen verschiedener Spurenstoffe an einem Punkt in der Atmosphäre. Diese einfache Messanordnung (im Vergleich zu einer mindestens zweidimensionalen Erfassung von Windkomponenten und Spurenstoffkonzentrationen) ist möglich, da entsprechend der Taylor-Hypothese die an einem Punkt in situ ${ }^{8}$ aufgenommenen Zeitreihen auch die räumliche Verteilung der untersuchten Größen repräsentieren (z.B. KAIMAL \& FINNIGAN, 1994).

Für die Windmessungen werden Ultraschall-Anemometer (sogenannte Sonics), Laser- oder Hitzedraht-Anemometer, bei Messungen in größeren Höhen auch Schalenkreuz-Anemometer verwendet. Lufttemperaturen werden in der Regel mit Thermoelementen oder ebenfalls mit Sonics, Partikelkonzentrationen mit optischen Partikeldetektoren und Luftfeuchten sowie Spurengaskonzentrationen mit zeitlich hochauflösenden Gasanalysatoren bestimmt. Bei letzteren unterscheidet man open path -Systeme, die die Messungen in situ durchführen, von closed path-Systemen, bei denen Probeluft für die Konzentrationsmessungen in abgeschlossene Gas- oder Partikelanalysatoren gepumpt wird.

Für die Berechnung der vertikalen Flüsse von Masse und Energie werden die gewonnenen Zeitreihen von Temperatur, Wasserdampf und Spurenstoffkonzentrationen mit den Zeitreihen der vertikalen Windgeschwindigkeit multipliziert:

$$
F=w \cdot \phi
$$

mit: $w$ vertikale Komponente des Windvektors

$\phi$ skalare Eigenschaft der Atmosphäre

Da Luftbewegungen und Stofftransporte in der planetarischen Grenzschicht der Atmosphäre (fast) ausschließlich turbulent erfolgen, können die Momentanwerte von $w$ und $\phi$ zu diesem

\footnotetext{
${ }^{8}$ in situ (lat.): in der Lage
} 
Zweck in je einen mittleren und in einen turbulenten Anteil zerlegt werden ("ReynoldsZerlegung"):

$$
\begin{aligned}
& w=\bar{w}+w^{\prime} \\
& \phi=\bar{\phi}+\phi^{\prime}
\end{aligned}
$$

$$
\begin{aligned}
\text { mit: } \Phi, w & \text { mittlere Anteile } \\
\Phi^{\prime}, w^{\prime} & \text { turbulente Anteile }
\end{aligned}
$$

Setzt man (14) in (13) ein und bildet das zeitliche Mittel über ein geeignetes Mittelungsintervall, so erhält man die mittlere Eigenschaftsflussdichte:

$$
\begin{aligned}
& F=\overline{\left(\bar{w}+w^{\prime}\right)\left(\bar{\phi}+\phi^{\prime}\right)} \\
& \Rightarrow F=\bar{w} \bar{\phi}+\bar{w} \overline{\phi^{\prime}}+\bar{w} \bar{\phi}+\overline{w^{\prime} \phi^{\prime}}
\end{aligned}
$$

Da die Mittel der turbulenten Anteile immer Null sind, vereinfacht sich (15) zu:

$$
F=\bar{w} \bar{\phi}+\overline{w^{\prime} \phi^{\prime}}
$$

Unter der Annahme, dass die Strömung im Mittel nur horizontal verläuft und somit der mittlere Vertikalwind ebenfalls Null ist (und damit Stofftransporte nur turbulent erfolgen), folgt:

$$
F=\overline{w^{\prime} \phi^{\prime}}
$$

Gleichung (17) beschreibt den turbulenten Fluss nur dann korrekt, wenn die Turbulenz stationär ist und das Mittelungsintervall für die Reynolds-Zerlegung so groß ist, dass auch alle niedrigfrequenten Anteile der turbulenten Strömung erfasst werden. BALDOCCHI ET AL. (1988) diskutieren die notwendige Länge des Intervalls und geben für eine Zeitspanne von 2000 Sekunden $(\approx 33$ Minuten) eine Fehlabschätzung der turbulenten Flüsse von 10 Prozent an. Dieser Betrag ließe sich theoretisch durch längere Mittelungsintervalle reduzieren, was praktisch aber kaum möglich ist, da dann die geforderte Stationarität (siehe unten) der Messreihen in der Regel nicht mehr gegeben ist und andere, nicht turbulente dynamische Effekte (z.B. der Tagesgang der betrachteten Eigenschaft) die Messungen verfälschen würden.

Gleichung (17) ist Ausgangspunkt für die Berechnung vertikaler Stoff- und Energieflüsse mit Hilfe der Eddy-Kovarianzmethode. Es ist wichtig festzuhalten, dass immer nur der turbulente Anteil von $F_{w}$ erfasst werden kann. Stromdichten mit einer mittleren vertikalen Strömung werden dagegen nicht berücksichtigt. 
Anwendung findet die Methode in der Mikrometeorologie traditionell bei der Bestimmung des vertikalen Impulsflusses $\tau$ :

$$
\tau=\rho \cdot \overline{w^{\prime} u^{\prime}},
$$

mit: $\rho$ Dichte feuchter Luft $\left(\mathrm{kg} / \mathrm{m}^{3}\right)$

$u$ Longitudinale Komponente des Windvektors $(\mathrm{m} / \mathrm{s})$

bei der Bestimmung des vertikalen fühlbaren Wärmeflusses $H$ :

$$
\begin{gathered}
H=\rho c_{p} \cdot \overline{w^{\prime} T^{\prime}}, \\
\text { mit: } c_{p} \text { Spezifische Wärmekapazität der Luft }(\mathrm{J} / \mathrm{K} \mathrm{kg}) \\
T \quad \text { Lufttemperatur }(\mathrm{K})
\end{gathered}
$$

sowie bei der Bestimmung des vertikalen latenten Wärmeflusses $L E$ :

$$
L E=\rho \lambda_{w} \overline{\cdot w^{\prime} q^{\prime}}
$$

mit: $\lambda_{w}$ Spezifische Verdampfungswärme von Wasser $(\mathrm{J} / \mathrm{kg})$

$q$ Spezifische Feuchte $(\mathrm{g} / \mathrm{kg})$

Mit Hilfe von Konzentrationsfluktuationen können darüber hinaus auch die vertikalen Flüsse von (gasförmigen) Spurenstoffen bestimmt werden nach:

$$
F_{S G}=K \overline{w^{\prime} c_{S G}^{\prime}}
$$

$$
\begin{aligned}
\text { mit: } F_{S G} & \text { Vertikale Flussdichte eines gasförmigen Spurenstoffes } \\
c_{S G} & \text { Konzentration des betrachteten Stoffes } \\
K & \text { Konstante in Abhängigkeit von den Einheiten von } c_{S G} \text { und } w
\end{aligned}
$$

wie dies beispielsweise bereits mehrfach für $\mathrm{CO}_{2}$ (z.B. IBRoM, 1999), für Ozon (z.B. NEUMANN \& HARTOG, 1985) und für Schwefel (z.B. HicKS ET AL., 1982) durchgeführt wurde.

\subsubsection{Voraussetzungen für die Durchführung der Eddy - Kovarianzmethode}

Für die Durchführung der Eddy - Kovarianzmethode werden im allgemeinen die folgenden Voraussetzungen genannt: horizontale Homogenität des untersuchten Standorts, Quasistationarität des Turbulenzfeldes, vernachlässigbare Dichteschwankungen sowie die Verfügbarkeit schneller und zuverlässiger Messinstrumente für eine vollständige Erfassung der Fluktuationen der betrachteten Eigenschaft der Atmosphäre (MCMILLEN, 1988; FOKEN \& WichURA, 1996). Diese Punkte, die beispielsweise auch die Einhaltung einer bestimmten Messhöhe implizieren, sollen im folgenden diskutiert werden; die Grenzen der Kovarianzmethode werden dabei aufgezeigt. 
Mikrometeorologische Methoden zur Bestimmung des vertikalen Partikelflusses

\section{a) Horizontale Homogenität}

Flüsse, die über die Eddy - Kovarianzmethode gemessen werden, repräsentieren immer eine räumlich-integrative Größe als Resultat einer Vielzahl von Quellen und/oder Senken in einem mehr oder weniger großen Einzugsbereich, der als Quellfläche (source area, footprint) bezeichnet wird. Diese Quellfläche ist im wesentlichen eine Funktion der Rauhigkeit der betrachteten Oberfläche, der Messhöhe, der Windgeschwindigkeit und Windrichtung sowie der Stabilität der Atmosphäre und damit nicht notwendigerweise über den Zeitraum einer Messung konstant. Durch die sogenannte Footprint-Funktion (SCHUEPP ET AL., 1990) bzw. Quellgewichtsfunktion (SCHMID \& OKE, 1988) kann die Quellfläche hinsichtlich ihrer räumlichen Verteilung und Intensitätsverteilung beschrieben werden.

Für eine sinnvolle Interpretation der Messresultate ist es erforderlich, innerhalb der Quellfläche von einer horizontalen Homogenität ausgehen zu können. Dafür ist es notwendig, ausgehend von einem bestimmten Messstandort und in Richtung des jeweiligen Windes jeweils diejenige Strecke zu definieren, entlang der eine gleiche Beschaffenheit der Oberfläche vorherrscht. Diese Strecke wird als fetch bezeichnet. Überdeckt der fetch für alle Richtungen die Quellfläche vollständig, repräsentieren die Messungen an einem Punkt die turbulenten Flüsse für die gesamte Fläche.

Da die Quellfläche einer Messung um so kleiner wird, in je geringerer Höhe die Messung durchgeführt wird, lässt sich scheinbar ableiten, dass für Eddy-Kovarianzmessungen - insbesondere bei nur geringen Dimensionen des fetches - möglichst niedrige Messhöhen anzustreben sind. Dies wird jedoch durch die Existenz der Rauhigkeitsschicht über dem Bestand eingeschränkt, in der die vertikalen Stoffflüsse auf Grund der kleinräumigen Heterogenität der Unterlage nicht mehr zuverlässig ermittelt werden können. Kann die Positionierung der Messinstrumente nicht so durchgeführt werden, dass die betrachtete Quellfläche der Bedingung der horizontalen Homogenität genügt und gleichzeitig die Messhöhe oberhalb der Rauhigkeitsschicht liegt, sind Eddy-Kovarianzmessungen nur eingeschränkt möglich.

\section{b) Quasistationarität des Turbulenzfeldes}

Unter Stationarität versteht man die Homogenität in der Zeit; das heißt, die Unabhängigkeit der statistischen Eigenschaften einer untersuchten Größe von der Zeit selbst. Diese Unabhängigkeit ist jedoch für meteorologischen Größen in der bodennahen Luftschicht höchst selten gegeben.

Allein der unvermeidliche natürliche Tagesgang vieler Größen stellt eine klare Instationarität dar. Um diesen zu eliminieren und die unregelmäßigen turbulenten Schwankungen, die für den turbulenten Transport verantwortlich sind, aus dem allgemeinen Trend herauszufiltern, werden die Mittelungsintervalle für die Reynolds-Zerlegung auf 30 bis maximal 60 Minuten begrenzt. Außerdem wird von jeder Zeitreihe nicht nur das arithmetische Mittel, sondern auch der lineare Trend eliminiert. Damit wird z. B. der Momentanwert $w$ der vertikalen Komponente des Windvektors folgendermaßen zerlegt: 
Mikrometeorologische Methoden zur Bestimmung des vertikalen Partikelflusses

$$
\begin{gathered}
w=\left(a_{w}+b_{w} t\right)+w^{\prime}, \\
\text { mit: } a_{w}^{\prime} b_{w} \text { Regressionskoeffizienten }
\end{gathered}
$$

$a_{w}$ und $b_{w}$ werden innerhalb des Mittelungsintervalls berechnet, analog wird mit allen Größen verfahren (EUGSTER, 1994). Mit dieser Berechnungsvorschrift wird gleichzeitig auch ein möglicher Drift von Messsensoren korrigiert, wie er beispielsweise durch eine sich ändernde Umgebungstemperatur verursacht werden kann. Ein ähnlicher Effekt kann mit der Verwendung eines high-pass Filters erreicht werden (SHUTTLEWORTH, 1987).

In Folge von Wetterumschwüngen und anderen meteorologischen Ereignissen können Instationaritäten meteorologischer Größen auch unabhängig vom Tagesgang auftreten. Dies ist insbesondere von Partikelkonzentrationsmessungen bekannt (z.B. BUZORIUS ET AL., 1998). Zur Absicherung der Stationarität der Turbulenz schlagen FOKEN \& WICHURA (1996) einen Test vor, bei dem eine Zeitreihe in 4 bis 8 Teilstücke aufgeteilt wird. Für jedes dieser Teilstücke wird die Kovarianz zweier Größen getrennt berechnet, anschließend werden die Ergebnisse gemittelt und mit der Gesamtkovarianz (berechnet aus der gesamten Zeitreihe) verglichen.

Im Falle von starken Instationaritäten ist der turbulente Fluss nicht mehr definiert, die Eddy Kovarianzmethode kann dann nicht mehr zur Flussberechnung verwendet werden (MCMillen, 1988).

\section{c) Vollständige Erfassung der Fluktuationen}

Jedes Messgerät kann Messungen nur mit einer bestimmten maximalen Messfrequenz ausführen, welche als cutoff-frequenz bezeichnet wird. Alle Fluktuationen der Messgröße, die mit einer höheren Geschwindigkeit stattfinden, werden von dem Messgerät nicht mehr erfasst. Es findet deshalb eine Dämpfung der Varianz des Signals im - relativ zum Charakter des Signals - hochfrequenten Bereich statt.

Während für Eddy-Kovarianzmessungen Sonics und Thermoelemente ${ }^{9}$ die Wind- und Temperaturfluktuationen heute in einer sehr hohen zeitlichen Auflösung erfassen können (10 Hz und mehr), genügen die zur Verfügung stehenden Gas- und Partikelanalysatoren häufig noch nicht den Anforderungen der Methode. Kann ein Analysator auf Grund seiner Trägheit nicht alle am turbulenten Transport beteiligten Fluktuationen der Gaskonzentration erfassen, wird aber auch der betrachtete Fluss nur unvollständig beschrieben.

Allgemein wird davon ausgegangen, dass für Eddy - Kovarianzmessungen die Zeitreihen der interessierenden Größen mit einer Messfrequenz von mindestens $10 \mathrm{~Hz}$ aufgenommen werden müssten, um den vertikalen Stoff- oder Energiefluss vollständig beschreiben zu können. So gibt EugSTER (1994) an, dass bei einer Messfrequenz von $1 \mathrm{~Hz}$ nur noch $90 \%$ des tatsächlichen Flusses erfasst werden würden, bei $0,1 \mathrm{~Hz}$ wären es nicht einmal mehr $50 \%$.

\footnotetext{
${ }^{9}$ Thermoelemente (engl.: thermocouples): einfache Temperatursensoren mit zwei Drähten aus unterschiedlichen Metallen (vgl. hierzu: PERRIN, 1999)
} 
Wie jedoch eigene Untersuchungen zeigen, kann bei Messungen über Wäldern auf Grund der höheren Rauhigkeit dieser Strukturen auch eine Messfrequenz von lediglich $1 \mathrm{~Hz}$ noch als ausreichend angesehen werden, da die am turbulenten Transport beteiligten eddies größere Dimensionen aufweisen als über Grasflächen (Abb. 4). Für Geräte, die auch diese Messfrequenz nicht mehr erfassen können, ist die Anwendung der Eddy - Kovarianzmethode nur noch eingeschränkt und in Kombination mit entsprechenden Korrekturen (siehe Abschnitt 2.2.4) möglich.
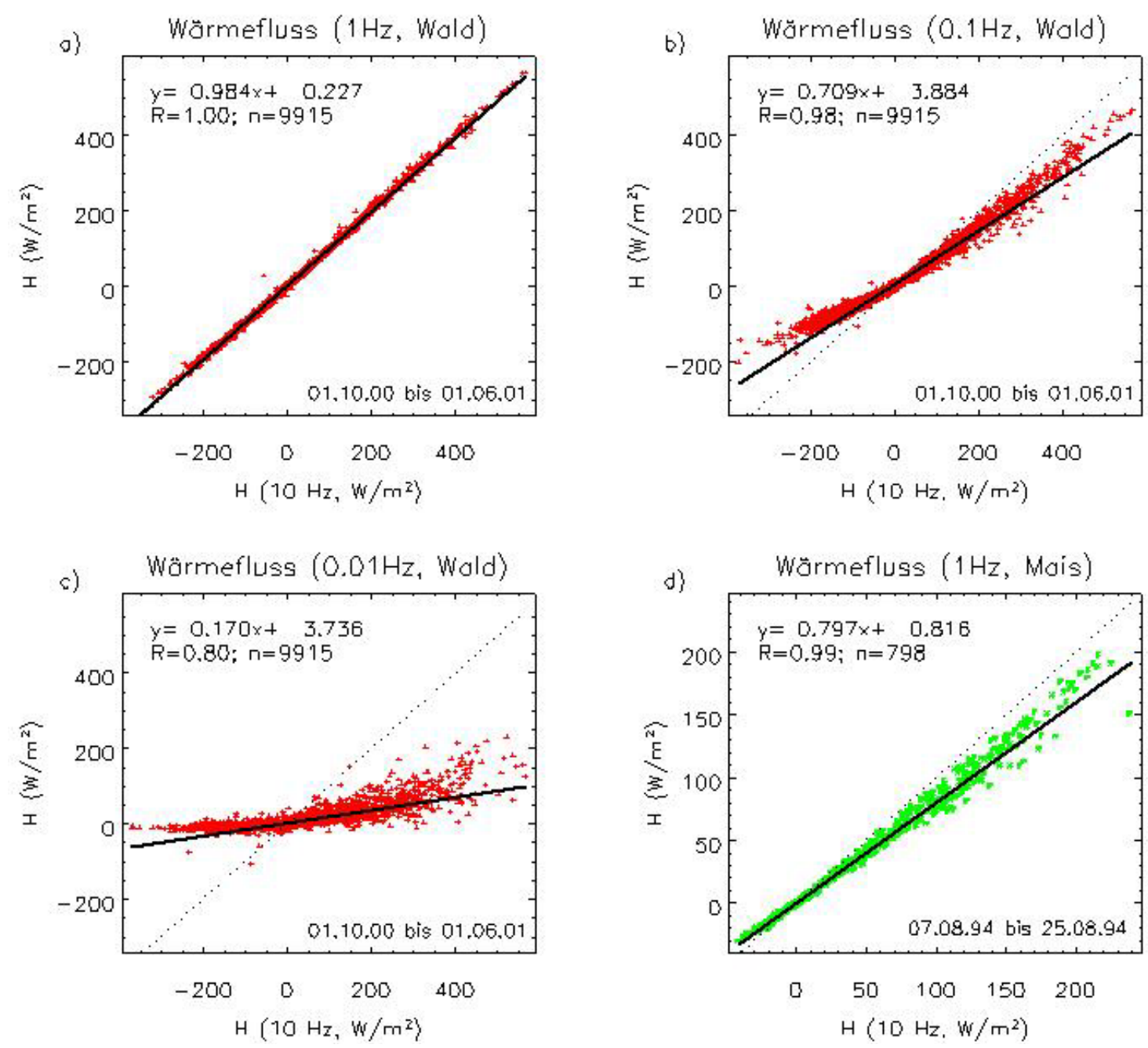

Abb. 4: Berechnete Halbstunden-Werte des fühlbaren Wärmeflusses über Wald aus $1 \mathrm{~Hz}, 0,1 \mathrm{~Hz}$ und $0,01 \mathrm{~Hz}$ Daten als Funktion der Resultate aus $10 \mathrm{~Hz}-$ Daten (Abb. a bis c, eigene Daten). Zum Vergleich die Resultate aus $1 \mathrm{~Hz}$ Daten für eine Messung über Mais (Abb. d; unveröffentlichte Daten des Instituts für Bioklimatologie, Universität Göttingen). Während über Mais bereits bei $1 \mathrm{~Hz}$ signifikante Unterschiede gegenüber den Werten aus $10 \mathrm{~Hz}$ zu erkennen sind, liefern Eddy - Kovarianzmessungen über Wald auch bei einer Messfrequenz von nur $1 \mathrm{~Hz}$ noch sehr gute Ergebnisse.

Für eine zuverlässige Ermittlung der turbulenten Flüsse ist es darüber hinaus notwendig, die interessierenden meteorologischen Größen mit einem hohen Signal-Rauschen-Verhältnis 
Mikrometeorologische Methoden zur Bestimmung des vertikalen Partikelflusses

aufzuzeichnen. Diese Voraussetzung ist insbesondere im Falle sehr kleiner Flüsse zwischen Atmosphäre und Erdoberfläche und damit verbunden sehr kleiner Fluktuationen der atmosphärischen Größen (z.B. bei stabiler Schichtung in der Nacht) nicht immer gegeben. Für eine Korrektur werden die gemessenen (unvollständigen) Flüsse häufig nach dem Kriterium der Schubspannungsgeschwindigkeit aussortiert und durch modellierte Werte ersetzt. Dieses Verfahren setzt jedoch bereits modellhafte Kenntnisse über die Natur der betrachteten Flüsse voraus.

\subsubsection{Eddy - Kovarianzmethode mit Partikeln}

Bereits Ende der 70er Jahre entstand die Idee, die Eddy - Kovarianzmethode auch für die Bestimmung von Partikelflüssen einzusetzen (WESELY ET AL., 1977). Im Vergleich zu anderen untersuchten Stoffen wurden jedoch bis heute nur relativ wenige Messungen durchgeführt. Die meisten veröffentlichten Studien sind Untersuchungen von nur wenigen Tagen Dauer. In etlichen Arbeiten wurden lediglich sehr niedrige Flüsse mit großen Unsicherheiten bestimmt, vielfach sind die aufgenommenen Zeitreihen der Partikelkonzentration stark verrauscht (Tabelle 4).

Die technische Umsetzung der Messungen der Partikelkonzentration wird von den einzelnen Autoren sehr unterschiedlich gehandhabt. Die Messungen sind also nicht standardisiert, wie dies beispielsweise bei Messungen zum $\mathrm{CO}_{2}$ Haushalt de facto der Fall ist. Es werden sowohl unterschiedliche Analysatoren als auch unterschiedliche Prinzipien beim Ansaugen der Partikel ein- bzw. umgesetzt. Die Realisierung des Ideals des isokinetischen Ansaugens der Probenluft über eine Steuerung der Ansauggeschwindigkeit ist für eine Eddy Kovarianzmessung praktisch nicht umsetzbar, da dies Artefakte bei der Kovarianzberechnung nach sich ziehen würde (NEUMANN \& HARTOG, 1985). Alternativ wäre eine kontinuierliche Regulierung der Größe der Ansaugöffnung denkbar, was eine enorme technische Herausforderung darstellt. In Anbetracht der Tatsache, dass die Beträge der Partikelflüsse im allgemeinen mit der Partikelkonzentration selbst normiert werden, wird darauf üblicherweise verzichtet.

Im Gegensatz zu Gasmolekülen sind Partikel in einem stärkeren Maße der Sedimentation unterworfen und werden somit nicht ausschließlich turbulent transportiert. Deshalb kann mit der Eddy - Kovarianzmethode nur ein Teil des vertikalen Partikelflusses bestimmt werden. Der Gesamtfluss ergibt sich aus dem turbulenten Anteil und einem zusätzlichen Term für den Sedimentationsvorgang (DUAN ET AL., 1988):

$$
\begin{array}{cl}
F=\overline{w^{\prime} c^{\prime}}-v_{g} \bar{c} \\
\text { mit: } c \text { Partikelkonzentration }\left(1 / \mathrm{cm}^{3}\right) \\
v_{g} \quad \text { Sedimentationsgeschwindigkeit }(\mathrm{m} / \mathrm{s})
\end{array}
$$

In den aufgeführten Arbeiten wurden immer wieder aufwärtsgerichtete Partikelflüsse beobachtet (z.B. Buzorius ET AL., 1998; GALlaGHER ET AL., 1997), deren Auftreten eine 
Schwierigkeit bei der Interpretation der Ergebnisse darstellen. Im allgemeinen werden sie als zeitweise Emission von in der oder kurz über der Vegetation neugebildeter Partikel gedeutet. Diese Partikel entstehen aus verschiedenen, durch die Vegetation emittierten Hydrokarbonaten (WENT, 1960).

Tabelle 4: Ausgewählte Eddy - Kovarianzmessungen mit Partikeln über verschiedenen Oberflächen

\begin{tabular}{|c|c|c|c|c|c|}
\hline Autoren & Dauer ${ }^{a)}$ & $\begin{array}{l}\text { Radius } \\
(\mu \mathrm{m})\end{array}$ & Messprinzip & $\begin{array}{l}\text { Ober- } \\
\text { fläche }\end{array}$ & Bemerkungen \\
\hline $\begin{array}{l}\text { KATEN \& HUBBE } \\
(1985)\end{array}$ & 1 & $0,15 . .2,5$ & $\mathrm{OPC}^{\mathrm{b})}$ & Gras & $\begin{array}{l}\text { Power Spektren in hohen Frequenzen von } \\
\text { Rauschen dominiert, starke Variabilität (positiv } \\
\text { und negativ) der Flüsse }\end{array}$ \\
\hline $\begin{array}{l}\text { NEUMANN \& } \\
\text { HARTOG (1985) }\end{array}$ & 1,5 & $0,09 . .2,67$ & OPC & Gras & $\begin{array}{l}\text { Standardfehler für berechnete } \\
\text { Depositionsgeschwindigkeiten größer als diese } \\
\text { selbst, Spektren ab 0,3 Hz stark verrauscht; } \\
\text { größenspezifische Messungen }\end{array}$ \\
\hline $\begin{array}{l}\text { WESELY ET AL. } \\
(1985)\end{array}$ & mehrere & $0,05 . .1,0$ & $\begin{array}{l}\text { Flame } \\
\text { photometric } \\
\text { detector }\end{array}$ & Gras & $\begin{array}{l}\text { starke Variabilität des gemessenen Flusses im } \\
\text { Tagesverlauf, durch Autoren auf statistisches } \\
\text { Rauschen der Partikelzählers zurückgeführt }\end{array}$ \\
\hline \multirow[t]{2}{*}{$\begin{array}{l}\text { DUAN ET AL. } \\
(1988)\end{array}$} & \multirow[t]{2}{*}{1} & $0,15 . .3$ & OPC & Schnee & $\begin{array}{l}\text { starkes Rauschen ab } 0,1 \mathrm{~Hz} \text {, hoch variable } \\
\text { Kovarianz mit } z \text { (ebenfalls stark verrauscht) }\end{array}$ \\
\hline & & $0,5 . .1,0$ & OPC & Schnee & $\begin{array}{l}\text { akzeptable Spektren mit deutlich verschobenem } \\
\text { Maximum bei hoher Ansauggeschwindigkeit, } \\
\text { trotzdem nur schwache Korrelation mit } w\end{array}$ \\
\hline $\begin{array}{l}\text { LAMAUD ET AL. } \\
(1994 b)\end{array}$ & 1 & $0.05 . .1$ & Charger & Wald & $\begin{array}{l}\text { Spektren zeigen kein Rauschen, zeitweiliges } \\
\text { Auftreten von Instationaritäten }\end{array}$ \\
\hline $\begin{array}{l}\text { GALLAGHER ET } \\
\text { AL. (1997) }\end{array}$ & 1 & $0,05 . .0,5$ & OPC & Wald & $\begin{array}{l}\text { größenspezifische Messungen; Größenklassen } \\
>0,5 \mu \mathrm{m} \text { Durchmesser wegen hoher } \\
\text { statistischer Unsicherheit nicht berücksichtigt }\end{array}$ \\
\hline $\begin{array}{l}\text { BUZORIUS ET AL. } \\
(1998)\end{array}$ & 6 & $0,012 . .10$ & $\mathrm{CPC}^{\mathrm{c})}$ & Wald & $\begin{array}{l}\text { Rauschen ab } 2 \mathrm{~Hz} \text {, jedoch deutlich weniger als } \\
\text { bei den anderen Arbeiten }\end{array}$ \\
\hline
\end{tabular}

\subsubsection{Korrekturen für die Eddy - Kovarianzmethode}

Während die Eddy - Kovarianzmethode einerseits als eine einfache und direkte Möglichkeit bezeichnet werden kann, turbulente Flüsse zu messen, birgt sie andererseits die Gefahr einer 
Reihe von zum Teil nicht vermeidbaren, systematischen Fehlern. Dies hat die Notwendigkeit von Korrekturen zur Folge bezüglich

- der gemessenen Komponenten des Windvektors (Flow-Distortion-Korrekturen, Koordinatenrotation),

- des Zeitversatzes bei closed path Analysatoren (Synchronisation der Messungen),

- einer möglichen Dämpfung der Fluktuationen der betrachteten Eigenschaft im Signal sowie

- des Einflusses der Fluktuationen von Temperatur und Luftfeuchte auf die Bestimmung der vertikalen Flüsse atmosphärischer Spurenstoffe (Webb-Korrektur).

Diese Korrekturen werden im folgenden insbesondere im Hinblick auf Eddy - Kovarianzmessungen mit Partikeln diskutiert.

\section{a) Korrektur der Messwerte des Ultraschallanemometers: Flow Distortion}

Eine Grundvoraussetzung der Eddy - Kovarianzmethode ist die Erfassung der vertikalen Windgeschwindigkeit mit hinreichender Genauigkeit. Die für diesen Zweck zum Einsatz kommenden Ultraschallanemometer beeinflussen den Windvektor jedoch in Abhängigkeit von ihrer Sensorgeometrie selbst mehr oder weniger stark, so dass hier gerätespezifische Korrekturen notwendig sind.

Für das in der vorliegenden Arbeit verwendete Sonic (USAT 3/1, vgl. Kapitel 3) wurde deshalb im Rahmen dieser Arbeit eine Windkanal-Kalibrierung durchgeführt, die ausführlich im Anhang beschrieben wird. Wie Abb. 5 zeigt, sind die Effekte einer solchen Korrektur erheblich: die Windgeschwindigkeit wird um $15 \%$ und der fühlbare Wärmefluss (und damit auch der Partikelfluss) sogar um fast $25 \%$ korrigiert.
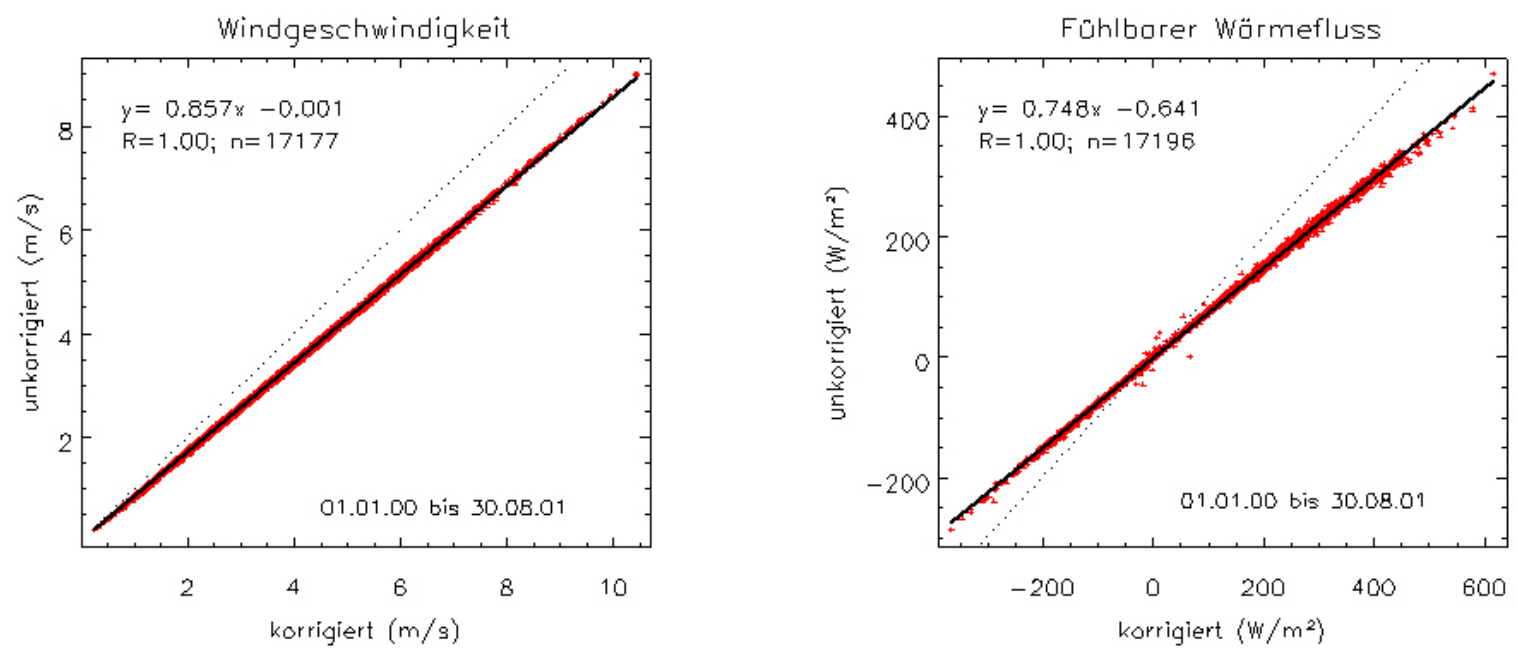

Abb. 5: Ausgewählte Effekte der Korrektur der Messwerte des Ultraschallanemometers 


\section{b) Korrektur der Messwerte des Ultraschallanemometers: Koordinatenrotation}

Das Produkt aus Luftdichte und der Kovarianz $\overline{w^{\prime} \phi^{\prime}}$ beschreibt den vertikalen Fluss der Grösse $\Phi$ nur dann vollständig, wenn der mittlere Vertikalwind $\bar{w}$ gleich Null ist. Obwohl diese Voraussetzung - mit Ausnahme eines sehr kleinen, mittleren Vertikalwindes - bei einer ausreichend langen Mittelungszeit eigentlich immer gegeben sein sollte, werden im Rahmen von mikrometeorologischen Messungen unter Freilandbedingungen hiervon abweichend mittlere Vertikalwinde größer oder kleiner Null beobachtet. Die eindeutige Zuordnung einer Ursache für diese Beobachtungen ist oft nicht möglich. Denkbar ist jedoch, dass das Windmessgerät nicht genau lotrecht montiert werden konnte oder dass Masteffekte das Windfeld verändert haben (VOGT, 1995).

Unter der Annahme, dass es sich in jedem Fall um Artefakte handelt, werden die gewonnenen Daten mit Hilfe einer Koordinatenrotation in der Weise manipuliert, dass das Koordinatensystem des Sonics nachträglich an die Stromlinien des Turbulenzfeldes angepasst wird. Bei dieser Rotation wird die Matrix des Windvektors so gedreht, dass sowohl die mittlere vertikale als auch die mittlere laterale Komponente des Vektors Null beträgt (MCMILLEN, 1988).

Die Koordinatenrotation kann entweder vor oder nach der Flussberechnung durchgeführt werden. Sie erfolgt in zwei Schritten: Zuerst wird das Koordinatensystem um die Vertikalachse in Richtung des mittleren Horizontalwindes gedreht. Der Drehwinkel errechnet sich aus:

$$
\alpha=\arctan (\bar{v} / \bar{u})
$$

wobei die Rotationsmatrix die Form

$$
D 1=\left(\begin{array}{ccc}
\cos \alpha & \sin \alpha & 0 \\
-\sin \alpha & \cos \alpha & 0 \\
0 & 0 & 1
\end{array}\right)
$$

hat. Im zweiten Schritt wird das Koordinatensystem so um die y-Achse gedreht, dass $\bar{w}$ gleich Null wird. Der Drehwinkel errechnet sich diesmal aus:

$$
\beta=\arctan \left(\frac{\bar{w}}{\sqrt{\bar{u}^{2}+\bar{v}^{2}}}\right),
$$

und die Rotationsmatrix hat die Form:

$$
D 2=\left(\begin{array}{ccc}
\cos \beta & 0 & \sin \beta \\
0 & 1 & 0 \\
-\sin \beta & 0 & \cos \beta
\end{array}\right)
$$


Mikrometeorologische Methoden zur Bestimmung des vertikalen Partikelflusses

\section{c) Synchronisation der Messungen}

Wird die Konzentration eines Spurenstoffes in der Atmosphäre mit einem closed path Sensor erfasst, wie dies bei Partikelmessungen immer der Fall ist, wird ein Transport der zu untersuchenden Luft in einem Ansaugschlauch bzw. Ansaugrohr zum Analysator notwendig, der in einer endlichen, von Null verschiedenen Zeit erfolgt. Dementsprechend muss das gemessene Konzentrationssignal rückdatiert werden, was in der Regel im Verlauf der späteren Auswertung der Eddy - Kovarianzdaten realisiert wird.

Die Schwierigkeit dabei ist die Bestimmung des tatsächlichen Zeitversatzes $\Delta t$, da dieser von der Ansauggeschwindigkeit abhängt und so bereits in Folge von Temperaturschwankungen im Verlaufe eines Tages variieren kann. Hier ist entweder eine Ableitung des Zeitversatzes aus der gemessenen Ansauggeschwindigkeit und dem Gesamtvolumen des Ansaugweges (z.B. TworeK, 1996) oder eine Bestimmung über die Kovarianzmaximierung möglich, bei der die Kovarianz $C o(w, \phi)$ für verschiedene Zeitversätze $\tau$ berechnet wird:

$$
\operatorname{Co}(w, \phi)=\sum_{t=0} w^{\prime}(t) \phi^{\prime}(t+\tau)
$$

Dabei wird angenommen, dass die in der Natur auftretende Kovarianz aus $w$ und $\phi$ immer maximal ist, so dass also gilt:

$$
\Delta t=\tau_{\max }(C o(w, \phi))
$$

Obwohl die Korrektheit dieses Ansatzes nicht nachgewiesen ist, handelt es sich bei der Kovarianzmaximierung um eine weit verbreitete und allgemein anerkannte Methode. Sie ist jedoch nur für Messreihen mit relativ großen, turbulenten Flüssen durchführbar, da nur hier ein deutliches Kovarianzmaximum ausgebildet ist.

\section{d) Dämpfungskorrektur}

Kann ein Analysator auf Grund seiner Trägheit nicht alle Fluktuationen eines Spurenstoffes in der Atmosphäre erfassen, kommt es zu einer mehr oder weniger starken, gerätespezifischen Dämpfung des tatsächlichen Signals, die eine Unterschätzung des betrachteten Stoffflusses nach sich ziehen kann. Zusätzlich werden bei closed path-Systemen hochfrequente Fluktuationen durch das Ansaugen der Luft in einem Ansaugrohr oder Schlauch verschmiert, da die Strömungsgeschwindigkeit in einer Röhre mit zunehmender Nähe zu den Wänden abnimmt. In einem turbulenten Regime sind die damit verbundenen Informationsverluste relativ niedrig und können praktisch vernachlässigt werden. Bei laminarer ${ }^{10}$ Ansaugung ist eine signifikante Unterschätzung des Flusses jedoch leicht möglich, wobei der Effekt von der Geometrie des Ansaugschlauchs, der Windgeschwindigkeit, der Messhöhe und weiteren Faktoren abhängig ist (PHILIP, 1963; LEUNING \& MONCRIEFF, 1990; MASSMAN, 1991; LENSCHOW \& RAUPACH, 1991; SUYKER \& VERMA, 1993).

\footnotetext{
${ }^{10}$ laminar: ohne Wirbel nebeneinander herlaufend
} 
Der Übergang einer laminaren in eine turbulente Strömung ist von der Zähigkeit des bewegten Mediums sowie von der Geschwindigkeit und der Größenskala der Bewegung abhängig. Als Maßzahl wird die sogenannte Reynoldszahl Re verwendet. Sie ist dimensionslos und wird bestimmt aus

$$
\operatorname{Re}=\frac{2 r U}{v}
$$

$$
\begin{aligned}
\text { mit: } r & \text { Radius des Ansaugrohrs / des Ansaugschlauchs }(\mu \mathrm{m}) \\
U & \text { ungestörte Geschwindigkeit im Ansaugrohr / Ansaugschlauch }(\mathrm{m} / \mathrm{s}) \\
\cup & \text { kinematische Zähigkeit von Luft }\left(1,510^{-5} \mathrm{~m}^{2} \mathrm{~s}^{-1} \text { bei } 0^{\circ} \mathrm{C} \text { und } 1013,2 \mathrm{hPa}\right)
\end{aligned}
$$

Für laminare Strömungen gilt $R e<2300$, für turbulente Strömungen entsprechend $R e>2300$.

$\mathrm{Ob}$ insgesamt tatsächlich Konzentrationssignale gedämpft worden sind, die zum turbulenten Fluss beigetragen haben, kann durch einen Vergleich der Kospektren mit den sogenannten "Kaimal-Spektren" (KAIMAL ET AL., 1972) oder alternativ mit den Spektren anderer, ungedämpfter Flüsse überprüft werden. Für letzteren Zweck kommt jedoch im wesentlichen nur die Lufttemperatur in Frage, da bei den üblichen Messverfahren nur für diese Größe angenommen werden kann, dass die am turbulenten Transport beteiligten Fluktuationen nahezu vollständig erfasst werden konnten.

Wird eine signifikante Dämpfung des Signals einer meteorologischen Größe festgestellt, kann diese bei der Berechnung der Flüsse durch einen entsprechenden Korrekturfaktor berücksichtigt werden. Bei dieser Korrektur werden gleichzeitig Unterschätzungen der turbulenten Flüsse korrigiert, die ggf. durch eine räumliche Separation einzelner Sensoren in Bezug zum Ultraschallanemometer und/oder durch zu kurze Mittelungsintervalle bei der ReynoldsZerlegung verursacht werden (MOORE, 1986; SUYKER \& VERMA, 1993; RIßMANN \& TETZLAFF, 1994; LEE \& BLACK, 1994).

\section{e) Webb-Korrektur}

BROOK (1978) hat darauf hingewiesen, dass die mit der Eddy - Kovarianzmethode erfassten Flüsse atmosphärischer Spurenstoffe bei Vorhandensein von Fluktuationen des atmosphärischen Wasserdampfs systematische Fehler gegenüber den realen Flüssen aufweisen. Dieses Problem ist mehrere Jahre lang intensiv diskutiert worden (WEBB \& PEARMAN, 1977; ReINKING, 1980; NiCHOLls \& SMITH, 1982; LeUNING ET AL., 1982; LEUNING \& LEGG, 1982; WEBB, 1982).

Allgemein anerkannt ist heute die von WeBB ET AL. (1980) für den Fall des Vorhandenseins eines fühlbaren oder latenten Wärmestroms - also für den Fall des Vorhandensein von mit $w$ korrelierten Dichtefluktuationen der Luft - vorausgesagte Existenz eines sehr kleinen, mittleren Vertikalwindes $\bar{w}$, der mit herkömmlichen Messinstrumenten nicht mehr messbar ist. Da dieser mittlere Vertikalwind jedoch u.U. mit einem Spurenstofftransport verbunden ist, wird eine Korrektur vorgeschlagen, bei der $\bar{w}$ modelliert wird nach: 
Mikrometeorologische Methoden zur Bestimmung des vertikalen Partikelflusses

$$
\begin{aligned}
\bar{w}_{\text {Modell }}=\mu\left(\overline{w^{\prime} \rho_{v}^{\prime}}\right) / \overline{\rho_{a}}+(1+\mu \sigma) \overline{w^{\prime} T^{\prime}} / \bar{T} \\
\text { mit: } \rho_{v} \text { Dichte von Wasserdampf }\left(\mathrm{kg} / \mathrm{m}^{3}\right) \\
\rho_{a} \text { Dichte trockener Luft }\left(\mathrm{kg} / \mathrm{m}^{3}\right) \\
\mu \text { Verhältnis der Molekulargewichte von Wasserdampf und trockener Luft }\left(0.622^{-1}\right) \\
\sigma \text { Verhältnis } \overline{\rho_{v}} / \overline{\rho_{a}}
\end{aligned}
$$

Die allgemeine Berechnungsanweisung für die Eddy - Kovarianzmethode (17) muss dann erweitert werden zu:

$$
F=w^{\prime} \phi^{\prime}+\bar{w}_{\text {Modell }} \bar{\phi}
$$

Die Existenz von $\bar{w}_{\text {Modell }} \neq 0$ konkretisiert die ursprünglich aufgestellten These und Voraussetzung der Eddy - Kovarianzmethode, der Vertikalwind sei im Mittel null (Abschnitt 2.2.1) in der Art, dass der mittlere Vertikalwind trockener Luft null ist.

Wenn die Spurenstoffkonzentrationen in Abwesenheit von Temperaturfluktuationen und/oder in trockener Luft gemessen werden, kann auf die sogenannte Webb-Korrektur ganz oder teilweise verzichtet werden. Bei einem closed path - System wird insbesondere die erste geforderte Voraussetzung durch die Dämpfung der Fluktuation der Lufttemperatur im Ansaugschlauch bzw. Ansaugrohr erfüllt (LEUNING \& MONCRIEFF, 1990; FAN ET AL., 1990; DENMEAD \& RAUPACH, 1993). Hierfür ist eine ausreichende Länge des Ansaugweges notwendig (ca. 1000facher innerer Durchmesser), die Art des verwendeten Materials spielt dagegen keine Rolle (RANNIK ET AL., 1997). Aus den genannten Gründen wird die WebbKorrektur bei Eddy - Kovarianzmessungen mit Partikeln nicht (z.B. BUZORIUS ET AL., 1998) oder nur teilweise (z.B. NEUMANN \& HARTOG, 1985) angewendet.

\subsection{Die Eddy - Akkumulationsmethode}

Da der Einsatz der Eddy - Kovarianzmethode auf Grund des Fehlens entsprechend schneller Messgeräte nicht für alle Spurenstoffe möglich ist, wurden alternative Möglichkeiten gesucht, die mit den Gradientmethoden verbundenen methodischen Probleme zu umgehen und die turbulenten Flüsse auf direktem Wege messen. Vor diesem Hintergrund wurde in den 70er Jahren die Eddy-Akkumulationsmethode entwickelt. Hier werden - der vertikalen Windgeschwindigkeit proportional - auf- und absteigende Luftvolumina in zwei getrennten Behältern gesammelt. Für ein gewisses Zeitintervall können dann die resultierenden Konzentrationsunterschiede zwischen den beiden Luftproben gemessen und in einen Massenfluss umgerechnet werden. Die praktische Umsetzung der Eddy-Akkumulationsmethode ist mit enormen technischen Problemen verbunden (HICKS \& MCMiLlen, 1984; SPEER ET AL., 1985; NEUMANN ET AL., 1989), so dass die Methode in dieser ursprünglichen Weise selten angewendet wurde. 
Mikrometeorologische Methoden zur Bestimmung des vertikalen Partikelflusses

Eine vereinfachte Variante wurde von BUSINGER \& ONCLEY (1990) entwickelt. Sie schlugen vor, die Ansaugmenge der Probenluft nicht mehr von der Geschwindigkeit, sondern nur noch von der Richtung des vertikalen Windes abhängig zu machen, die Probenluft also mit einer konstanten Flussrate anzusaugen. Diese Variante wird als relaxed eddy accumulation (REA) oder als conditional sampling bezeichnet. Der vertikale Stofffluss wird dann berechnet nach:

$$
F=\beta \sigma_{w}\left(\overline{C^{+}}-\overline{C^{-}}\right)
$$

$$
\begin{aligned}
\text { mit: } \sigma_{w} & \text { Standardabweichung der vertikalen Windgeschwindigkeit } \\
\frac{\beta}{C^{+}} & \begin{array}{l}
\text { Proportionalitätskonstante (dimensionslos, ca. } 0,56 \text { bis } 0,6) \\
\text { mittlere Spurenstoffkonzentration bei aufsteigendem Vertikalwind }
\end{array} \\
\overline{C^{-}} & \text {mittlere Spurenstoffkonzentration bei absteigendem Vertikalwind }
\end{aligned}
$$

Wichtig für die Anwendung der REA ist die Realisierung des raschen Umlenkens der Probeluft in den jeweilig "richtigen" Behälter. Während bei der Eddy - Kovarianzmethode die Messgeschwindigkeiten der Analysatoren die entscheidende Größe bei der Erfassung aller am turbulenten Fluss beteiligten Fluktuationen sind, gilt dies bei der REA in gleicher Weise für die Steuerung und Arbeitsgeschwindigkeit der Umlenkventile, die dementsprechend bis zu 5 bis 10 Umschaltvorgänge in der Sekunde realisieren müssen (XU, 2001). Für die Bestimmung der Proportionalitätskonstante werden verschiedene Varianten verwendet, beispielsweise kann diese empirisch über den fühlbaren Wärmefluss ermittelt oder aus dem Verhältnis zwischen Spurenstoffkonzentration und vertikalem Wind berechnet werden (BAKER, 2000).

REA ist erfolgreich eingesetzt worden für die Ermittlung vertikaler Flussdichten von $\mathrm{CO}_{2}$, $\mathrm{CH}_{4}, \mathrm{COS}$ und anderen Gasen (z.B. BEVERLAND ET AL., 1996; Guenther ET AL., 1996; MONCRIEFF ET AL., 1998; XU, 2001). Ein Einsatz für die Bestimmung der Deposition von Partikelflüssen scheint dagegen wenig sinnvoll, da diese zwischen dem Zeitpunkt des Eintreffens in den Sammelbehältern und dem Zeitpunkt der abschließenden Konzentrationsmessung wahrscheinlich im Behälter sedimentieren oder sich an den Wänden der Behälter absetzen würden.

\subsection{Bestimmung vertikaler Flussdichten atmosphärischer Partikel im Zuge der vorliegenden Arbeit}

Ziel der vorliegenden Arbeit war es, die Depositionsgeschwindigkeit luftgetragener Partikel als Funktion der Partikelgröße auf der F1-Fläche im Solling (siehe Kapitel 3) mit Hilfe mikrometeorologischer Methoden zu bestimmen. Hierfür stand ein einzelner, kommerziell erhältlicher, optischer Einzelpartikelzähler PCS-2000 der Firma Palas zu Verfügung. Die Anwendung der REA kam für den beabsichtigten Zweck aus den im vorherigen Abschnitt genanten Gründen prinzipiell nicht in Frage. Unter Berücksichtigung der Ergebnisse der Profilmessungen der Partikelkonzentration von CONSTANTIN (1993) auf der gleichen Untersuchungsfläche und auf Grund der Nichtverfügbarkeit eines zweiten Messgeräts wurde auch die Anwendung von Gradientmethoden ausgeschlossen. CONSTANTIN (1993) hat gezeigt, 
dass die in der atmosphärischen Grenzschicht über Wald vorhandenen Konzentrationsdifferenzen großen zeitlichen Schwankungen unterliegen und nur als Mittel über mehrere Monate auflösbar sind. Eine quantitative Bestimmung der Partikeldeposition aus solchen Daten ist mit aller größter Wahrscheinlichkeit nicht möglich. Einer Anwendung der Eddy Kovarianzmethode schien dagegen nichts im Wege zu stehen, so dass diese als Methode der Wahl für die vorliegende Arbeit angesehen wurde.

Für die Resultate der vorliegenden Arbeit wurden mittlere Depositionsgeschwindigkeiten von etwa $1 \mathrm{~cm} / \mathrm{s}$ erwartet, da dieser Wert den Ergebnissen der bereits auf der selben Fläche stattgefundenen Messungen zur Partikeldeposition entspricht. Die hohe zeitliche Auflösung der Ergebnisse der Eddy - Kovarianzmethode sollte genutzt werden, um die Abhängigkeit der Depositionsgeschwindigkeit von meteorologischen Randbedingungen zu klären. 


\section{Eigene Messungen zur Trockenen Deposition von luftgetragenen Partikeln: Messgelände und Setup}

In diesem Kapitel werden die eigenen Messungen zur Bestimmung der Depositionsgeschwindigkeit luftgetragener Partikel über einen Fichtenaltbestand vorgestellt. Zunächst wird der Messstandort - die sogenannte F1-Fläche im Solling - beschrieben, anschließend werden der Aufbau der Messanlage, die Datenaufnahme und die Datenverarbeitung dargestellt.

\subsection{Die F1-Fläche im Solling}

\subsubsection{Lage und Ausstattung der F1-Fläche}

Der Solling ist ein zwischen Weser und Leine, 60 - $80 \mathrm{~km}$ ssw. von Hannover und 30 - $60 \mathrm{~km}$ wnw. von Göttingen gelegenes und zum "Weserbergland" zugehöriges waldreiches Plateau im südlichen Niedersachsen. Er bildet eine exponierte, nach allen Seiten hin flach abfallende Erhebung von ungefähr $500 \mathrm{~km}^{2}$ Ausdehnung (Abb. 6). Sein höchster Punkt, die "Große Blöße" genannt, liegt $528 \mathrm{~m}$ über NN. Eine detaillierte Beschreibung der Region geben BEESE ET AL. (1999).

Abb. 6: Geographische Lage der Solling-Untersuchungsflächen (aus: ELLENBERG ET AL., 1986)

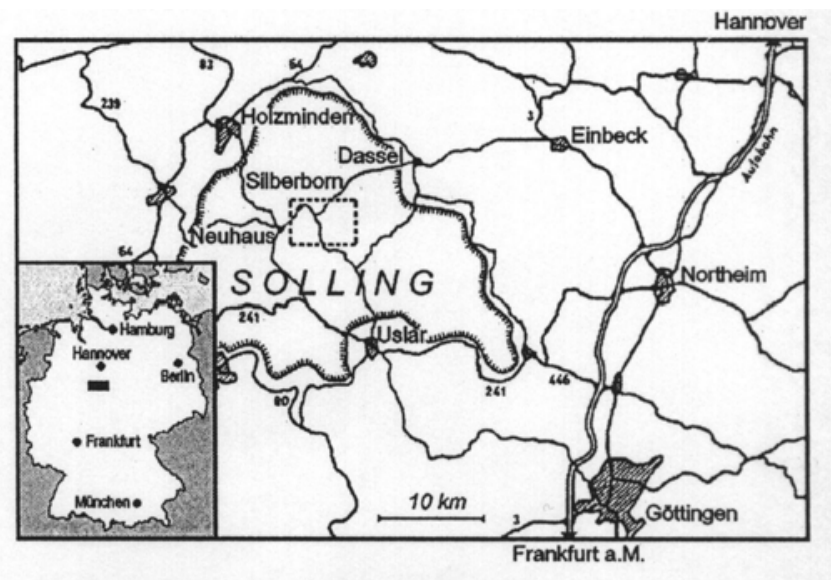

Seit über 30 Jahren werden im Solling Messungen zur Waldschadens- und Ökosystemforschung durchgeführt. Die Untersuchungen begannen 1966 im Rahmen des "Internationalen Biologischen Programms" (IBP) durch Wissenschaftler des Instituts für Geobotanik und des Instituts für Bodenkunde und Waldernährung der Universität Göttingen (ELLENBERG ET AL., 1986). Seither konnten in einer Reihe von interdisziplinären Projekten wichtige Erkenntnisse 
Eigene Messungen: Messgelände und Setup

zu langfristigen Entwicklungstrends in Wäldern gewonnen werden, die die Waldschadensund Ökosystemforschung in Deutschland nachhaltig beeinflusst haben.

Von den 1966 im Rahmen des IBP-Programms angelegten Untersuchungsflächen werden heute nur noch zwei Flächen im Bereich des sich über einem Durchmesser von $1300 \mathrm{~m}$ erstreckenden Hochplateaus des Sollings unterhalten. Eine dieser beiden Flächen ist mit Fichten, die andere mit Buchen bestanden - entsprechend werden sie als "F1" und "B1" bezeichnet. Beide Flächen liegen etwa 2,5 km östlich von Silberborn (ca. $2 \mathrm{~km}$ südlich der

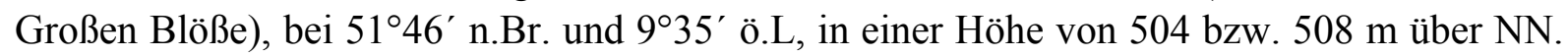
1992 wurden sie in das niedersächsische Dauerbeobachtungsprogramm (MEESENBURG ET AL., 1997), 1994 als Level II-Flächen in das Europäische Intensive Waldmonitoring (HAUBMANN \& LUX, 1997) aufgenommen. Die auf den beiden Waldflächen stattfindenden Untersuchungen dienen dem Ziel, den Stoff- und Energieaustausch von Ökosystemen am Beispiel des Fichtenund Buchenbestandes zu charakterisieren, Ursachen von Waldschäden zu determinieren und Prognosen für Auswirkungen von Veränderungen des physikalischen und chemischen Klimas zu erstellen. Die vorliegende, sich in diesen fachlichen Rahmen einfügende Arbeit ist auf der F1-Fläche entstanden.

Der geologische Untergrund der F1-Fläche wird durch Bundsandstein gebildet, welcher durch eine 40-80 $\mathrm{cm}$ dicke Schicht saurer, schwach podsoliger Braunerde bedeckt wird. Der nacheiszeitlich aus Löß entstandene Mineralboden trägt eine ca. $6 \mathrm{~cm}$ dicke Auflage aus Humus, das Gelände weist einen Anstieg von $1^{\circ} 20^{\prime}$ von Ost nach West auf. Das regionale Klima wird als subatlantisches Mittelgebirgsklima (Entfernung zur Nordseeküste ca. $250 \mathrm{~km}$ ) mit schneereichen Wintern und kühlen Sommermonaten charakterisiert. Wie fast überall im norddeutschen Klimaraum herrschen West- und Südwestwinde vor (Abb. 7). Die mittlere Niederschlagsmenge beträgt $1088 \mathrm{~mm} \mathrm{a}^{-1}$ mit zwei Maxima im Winter (Dezember - Januar) und im Sommer (Juli - August). Die durchschnittliche Jahrestemperatur liegt bei $6,4{ }^{\circ} \mathrm{C}$, Fröste sind bis in den Mai hinein möglich. Die höchsten Temperaturen von $27-28^{\circ} \mathrm{C}$ werden im Juli gemessen (EllenBerg ET AL., 1986; Constantin, 1993; BeESE ET AL., 1999).

Abb. 7: Klima des Sollings: Windrose für das Jahr 2000 auf der F1-Fläche (eigene Daten). Die Dominanz der Westwinde ist deutlich sichtbar.

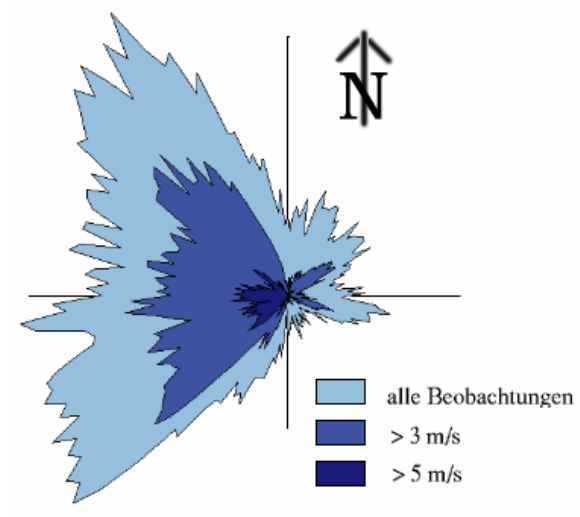

Die Vegetation besteht aus einem alten, homogenen Bestand von Gemeiner Fichte (Picea abies (L.) Karst., Abb. 8). Die Bäume wurden 1888 als damals vierjährige Setzlinge auf einer 
Eigene Messungen: Messgelände und Setup

ehemals beweideten Viehdrift gepflanzt und sind heute (im Jahr 2001) folglich 116 Jahre alt. Unter den Fichten hat sich eine für saure und nährstoffarme Standorte typische Flora entwickelt, unter einer spärlichen Strauchschicht gedeiht eine relativ üppige Krautschicht. Die mittlere Höhe der Bäume beträgt $30 \mathrm{~m}$, der mittlere Kronenansatz liegt bei $20 \mathrm{~m}$. Die Bestandsdichte beträgt 461 Bäume pro ha, der Blattflächenindex LAI (projiziert) ist 7. Die Verdrängungsschichtdicke $d$ wird mit $22,7 \pm 0,7 \mathrm{~m}$, der Rauhigkeitsparameter $z_{0}$ mit 2,5 \pm 0,1 m angegeben (ElLENBERG ET AL., 1986; LAUBACH ET AL., 1994; IBROM, 1999) ${ }^{11}$.

In nordwestlicher Richtung grenzt die Fläche an eine Freifläche (150 m Entfernung vom Messturm), in südlicher / südwestlicher (250 m vom Turm) und nordwestlicher $(600 \mathrm{~m}$ Entfernung vom Turm) Richtung an ausgedehnte, etwa gleichaltrige Buchenbestände (Fagus sylvatica, L.) von durchschnittlich $29 \mathrm{~m}$ Höhe, im Norden und Osten an weitere Fichtenbestände (mit kleinen Einstreuungen von Buchen im Osten). Der längste fetch hat eine Ausdehnung von $1000 \mathrm{~m}$ und befindet sich in nördlicher Richtung.
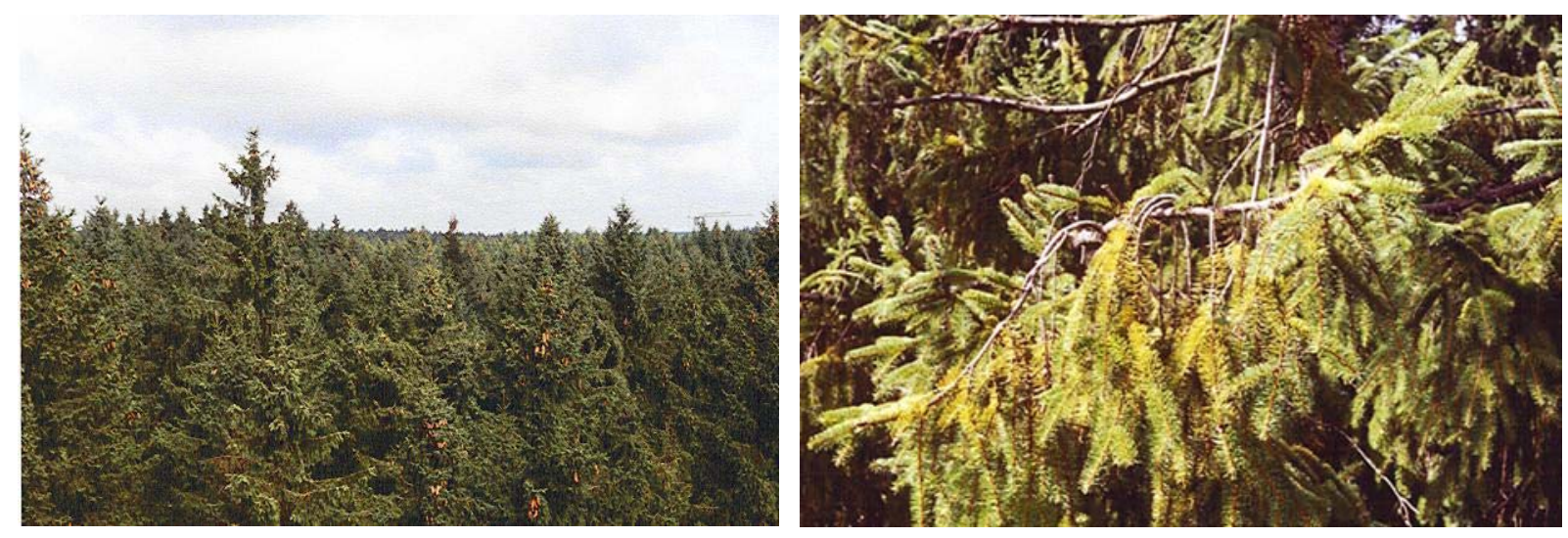

Abb. 8: Blick über die 116jährigen Fichten der F1-Fläche in Richtung Nord (linkes Foto) sowie Detailansicht eines Fichtenzweiges (rechtes Foto). Die Bäume sind sichtbar geschädigt und zeigen Symptome von Magnesiummangel.

Im Jahre 1989 wurde im Rahmen des Forschungsprojektes "Dynamik von Waldökosystemen" auf der F1-Fläche ein $52 \mathrm{~m}$ hoher Gittermastturm zur mikrometeorologischen Feldforschung errichtet. Der Turm hat einen quadratischen Grundriss von 2,5 $\mathrm{m}$ x 2,5 $\mathrm{m}$ und ist mit einem Lastenaufzug ausgestattet. Verschiedene meteorologische Parameter werden hier seit 1989 kontinuierlich in mehreren Höhen gemessen (Tabelle 5). Alle meteorologischen Daten werden zentral mit Hilfe eines Dataloggers (Firma Solartron) und eines PCs erfasst. Das Messintervall beträgt $10 \mathrm{~s}$, das Mitteilungsintervall $15 \mathrm{~min}$. Eine detailliertere Beschreibung der Messverfahren gibt z.B. BLANK (2000). Der Turm ist mit dem öffentlichen Stromnetz gekoppelt und besitzt einen Telefonanschluß, um Daten per Telefon übertragen zu können.

\footnotetext{
${ }^{11}$ MORGENSTERN (2000) findet dagegen für $z_{0} 1,28 \pm 0,83$ bzw. 1,99 $\pm 0,81 \mathrm{~m}$ und für $d 23,49 \pm 1,24$ bzw. 24,46 $\pm 1,38 \mathrm{~m}$.
} 
Eigene Messungen: Messgelände und Setup

Tabelle 5: Standardmeteorologie auf der F1-Fläche

\begin{tabular}{|l|l|l|}
\hline \multicolumn{1}{|c|}{ Messgröße } & \multicolumn{1}{c|}{ Messfühler } & \multicolumn{1}{c|}{ Messhöhe (m) } \\
\hline Globalstrahlung & Pyranometer (Kipp und Zonen, NL) & 2,39 \\
\hline Gesamtstrahlungsbilanz & Pyradiometer, nach Schulze (Lange, Berlin) & 2,39 \\
\hline $\begin{array}{l}\text { Lufttemperatur und } \\
\text { Luftfeuchtigkeit }\end{array}$ & $\begin{array}{l}\text { Psychrometer (Thies, mit am IBK } \\
\text { entwickelten NTC-Widerstandsfühlern) }\end{array}$ & $1,3,5,9,15,21,27,33$, \\
\hline Windgeschwindigkeit & Schalenkreuzanemometer (Thies) & $1,3,5,51,15,21,27,33$, \\
& & $39,45,51,56$ \\
\hline Windrichtung & Windfahnen & 35,52 \\
\hline Bodentemperatur & Pt-100-Widerstandsthermometer & $-2,-5,-10,-50,-100 \mathrm{~cm}$ \\
\hline Luftdruck & Druckdose (Spranger) & 2 \\
\hline Niederschlagsintensität & Ombrometer (Thies) & 27 \\
\hline
\end{tabular}

\subsubsection{Bisherige Eddy - Kovarianzmessungen und Untersuchungen zum Partikeleintrag auf der F1-Fläche}

Die F1-Fläche hat sich im Laufe der Zeit zu einer der wichtigsten Untersuchungsstandorte des Instituts für Bioklimatologie (IBK) der Universität Göttingen entwickelt. Neben der Erfassung der meteorologischen Standardparameter wurden auf der Fläche in den vergangenen Jahren bereits mehrere Eddy-Kovarianzmessungen und Untersuchungen zur Trockenen Deposition durchgeführt. Auf die Erfahrungen und die Ergebnisse dieser Messungen konnte bei der Bearbeitung der Fragestellung der vorliegenden Arbeit zurückgegriffen werden.

\section{a) bisherige Eddy-Kovarianzmessungen auf der F1-Fläche}

Der erste Aufbau eines Eddy-Kovarianzsystems zur Bestimmung von Wärme- und Wasserdampfflussdichten unter Verwendung eines Kaijo Denki- und eines USAT3-Sonics sowie einer Kryptonsonde erfolgte durch LAUBACH (1991) und RASCHENDORFER (1991). Beide führten Messungen in 39, 45 und 52 m Höhe über Boden durch. Im Mittelpunkt der Arbeit LAUBACHs stand dabei die Bestimmung der Wasserdampfflüsse, RASCHENFORFER dagegen konzentrierte sich auf allgemeine Aspekte der Turbulenz in und über dem Wald. LÖFFLER (1994) führte Eddy - Kovarianzmessungen ebenfalls in drei Messhöhen durch und beschäftigte sich mit der Schließung der Energiebilanz sowie mit notwendigen Korrekturfunktionen. RIESE (1995) testete vier Monate lang verschiedene Optionen für den Aufbau und den Betrieb der Anlage unter Verwendung zweier USAT3-Sonics in 45 und $9 \mathrm{~m}$ Höhe.

SCHÜTZ (1996) und TwOREK (1996) konzipierten im Jahr 1995 ein Eddy - Kovarianzsystem für direkte Flussmessungen von $\mathrm{CO}_{2}, \mathrm{H}_{2} \mathrm{O}$ und fühlbarer Wärme in einer Messhöhe von $39 \mathrm{~m}$. Der Messbetrieb (Beginn 1995/96) dieser Anlage wurde durch das IBK bis Ende 1999 fortgeführt. TWOREK führte darüber hinaus im Jahre 1996 erste Eddy - Kovarianzmessungen 
Eigene Messungen: Messgelände und Setup

von $\mathrm{CO}_{2}$ und $\mathrm{H}_{2} \mathrm{O}$ im Stammraum der F1-Fläche durch. FALK (1997) erneuerte die Anlage in $39 \mathrm{~m}$ Höhe, installierte zeitweilig ein zweites Sonic auf der gleichen Messplattform (gegenüberliegende Turmseite) und führte ebenfalls Messungen im Stammraum (nun in $2 \mathrm{~m}$ Höhe, ca. 20 m westlich des Turmes) durch. BLANK (2000) verglich schließlich Messungen des installierten closed-path $\mathrm{CO}_{2} / \mathrm{H}_{2} \mathrm{O}$-Systems mit einem open-path Krypton-Hygrometer.

Wichtige methodische und wissenschaftliche Ergebnisse der stattgefundenen EddyKovarianzmessungen sind:

1. Die erfassten vertikalen Energieflüsse in $39 \mathrm{~m}, 45 \mathrm{~m}$ und $52 \mathrm{~m}$ Höhe differieren nicht signifikant untereinander. Man befindet sich in diesen Messhöhen folglich im Bereich der constant flux layer (Prandtl-Schicht). Die bestimmenden Längenskalen des vertikalen, turbulenten Austauschs liegen zwischen $20 \mathrm{~m}$ und $1500 \mathrm{~m}$. Sie entsprechen Zeitskalen von etwa einer Viertelminute bis zu 15 Minuten. (LAUBACH, 1991; LÖFFLER, 1994).

2. Für die turbulenten Flüsse in $39 \mathrm{~m}$ Höhe liegen bei den tagsüber auftretenden instabilen Schichtungen die Flächen, aus denen der größte Teil des gemessenen Flusses stammt, auch für Wind aus der Richtung mit dem kürzesten fetch innerhalb des betrachteten Fichtenbestandes. Bei nächtlicher stabiler Schichtung kommt es dagegen zu einer Überlappung des Quellgebietes mit dem angrenzenden Buchenbestand, so dass dann die Ergebnisse von Eddy Kovarianzmessungen den betrachteten Bestand nicht mehr ausschließlich repräsentieren (IBROM, 1993).

3. Die mit der Eddy - Kovarianzmethode erhobenen Daten ergeben mit der Theorie übereinstimmende Spektren und Flüsse. Die Genauigkeit der Messungen wird bei der Flussbestimmung im wesentlichen durch die Genauigkeit der Analysatoren bestimmt (TwOREK, 1996).

4. Im Windschatten des Turms werden die vertikalen Flüsse deutlich unterschätzt (RIESE, 1995). Dabei kommt es zu einer Umlenkung von Vertikalfluktuationen der Strömung zu Horizontalfluktuationen, die auf der windabgewandten Seite zu einer Verringerung der gemessenen turbulenten Flüsse um bis zu 35 \% führen, während der Impulsfluss kaum verändert wird (FALK, 1997).

5. Die Energiebilanz kann durch die Ergebnisse der Eddy - Kovarianzmessungen nicht vollständig geschlossen werden. Der Betrag der Schließungslücke ist insbesondere bei stabiler Schichtung mit 30 bis $80 \%$ hoch, während unter labilen Verhältnissen eine Schließung von 90 bis $95 \%$ erreicht wird (IBROM, 1999).

\section{b) bisherige Untersuchungen zur Trockenen Deposition auf der F1-Fläche}

Auf der F1-Fläche wurden Untersuchungen zum Eintrag von atmosphärischen Partikeln bisher mit verschiedenen Methoden, jedoch noch nicht in Kombination mit der Eddy Kovarianzmethode durchgeführt: 
Eigene Messungen: Messgelände und Setup

- HöfKen \& Gravenhorst (1983) untersuchten die Trockene Deposition mit Kronentraufe- und Streufallanalyse.

- BRÜCKMANN ET AL. (1989) nutzten eine Radionuklidbilanzierung für eine Abschätzung der Depositionsgeschwindigkeit luftgetragener Partikel.

- Gravenhorst \& Waraghai (1990) bestimmten die trockene Depositionsgeschwindigkeit von luftgetragenen Partikeln unter Benutzung der Pflanzenoberfläche als Akzeptorfläche mit Hilfe von elektronenmikroskopischen Aufnahmen.

- Ibrom (1993) untersuchte die Deposition von Pflanzennährstoffen mit Hilfe der Kronentraufemethode.

- Constantin (1993) erfasste den Nebelwassereintrag und leitete aus den Spurenstoffkonzentrationen im Nebelwasser die Nebeldeposition ab; darüber hinaus erfasste er Anzahldichten des atmosphärischen Aerosol vom Waldboden bis über dem Kronenraum.

- MARQUes (1998) schließlich bestimmte die Depositionsgeschwindigkeit luftgetragener Partikel mit Hilfe von elektronenmikroskopischen Untersuchungen von Nadeloberflächen sowie mit dem Abwaschverfahren.

Abb. 9: Ergebnisse der bisherigen Depositionsmessungen auf der F1-Fläche (MMD: mittlerer Massendurchmesser; Quelle: GRAVENHORST ET AL., 2000)

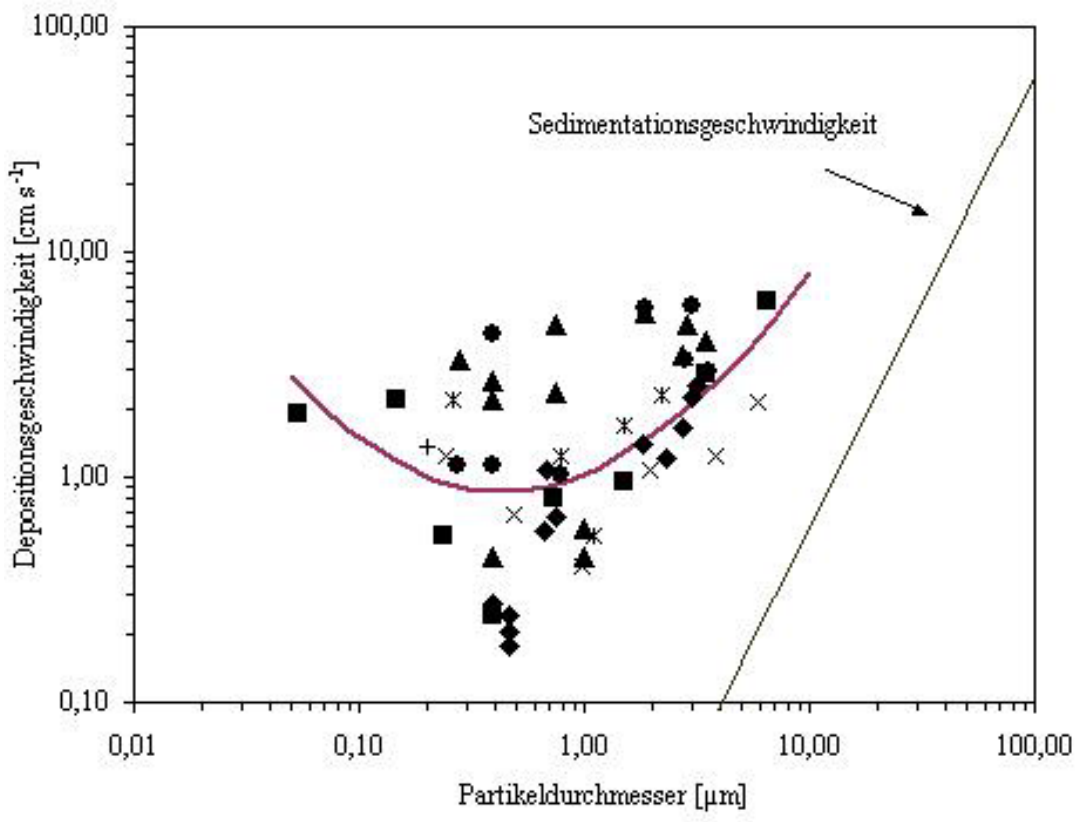

- Marques (1998): Abwaschverfahuren (MMD)

- Marques (1998): Elektronenurikroskop

A Ibrom (1993): Kronenraumbilanzienung (MMD)

$\times$ Graverhorst \& Waraghai (1990): Elektronerumikroskop

* Höfken et al. (1981): Kroneruraum- und Streubilanzierung (MMD)

- Constantin (1993): Nettokronentraufe (MMD)

+ Brückmann et al. (1989): Radionuklidbilanzienung 
Eigene Messungen: Messgelände und Setup

Die Resultate dieser bisherigen Depositionsmessungen auf der F1-Fläche sind in Abb. 9 zusammengefasst. Obwohl alle Messungen am gleichen Standort stattfanden, sind die Ergebnisse untereinander nur eingeschränkt vergleichbar, da die einzelnen Untersuchungen mit unterschiedlichen Methoden, bezogen auf unterschiedlich definierte Partikeldurchmesser und $\mathrm{zu}$ unterschiedlichen Zeiten - und damit unter unterschiedlichen meteorologischen Bedingungen - durchgeführt wurden.

\subsection{Aufbau der Messanlage}

\subsubsection{Zentrale Komponenten: PCS-2000 und USAT 3/1}

Die Messeinrichtung für die eigenen Messungen bestand im wesentlichen aus zwei Komponenten: einem Sonic zur Erfassung des vertikalen Windes und einem Partikelzähler zur Bestimmung der Partikelkonzentration.

Die vertikale Windgeschwindigkeit wurde mit einem Ultraschallanemometer USAT 3/1 der Firma Metek aufgezeichnet. Dieses "Hybridgerät" arbeitet mit einem Sondenkopf des USA1 und der elektronischen Steuerung des älteren Modells USAT3. Es ist seit längerer Zeit am IBK in Gebrauch und wurde deshalb bereits in zahlreichen Arbeiten beschrieben (z.B. GROS, 1998; BLANK, 2000).

Für die Bestimmung der Partikelkonzentration als Funktion der Partikelgröße stand ein kommerzieller Einzel-Partikelgrößenanalysator (PCS-2000, hiernach: OPC) der Firma Palas zur Verfügung. Dieses Gerät besteht aus drei optischen Teilsystemen: einem Beleuchtungssystem und zwei gegenüber angeordneten Detektorsystemen, deren optischen Achsen am Achsenschnittpunkt senkrecht zur optischen Achse des Beleuchtungssystems stehen (Abb. 10). Die atmosphärischen Partikel werden einzeln und nacheinander einem optisch abgegrenzten, homogen beleuchteten Messvolumen zugeführt, dort vermessen und in 128 Größenklassen gezählt. Die Bestimmung des Teilchendurchmessers erfolgt über die Analyse der Intensität des Streulichts, das von den Partikeln senkrecht zur Bestrahlungsrichtung abgegeben wird und von zwei Photomultipliern registriert wird. Als Lichtquelle wird eine Weißlichtquelle (Xenon-Hochdruck-Lampe) verwendet. Gegenüber monochromatischem Licht (Laser) ist Weißlicht durch einen relativ breiten Größenbereich der Partikel gekennzeichnet, in dem die Zuordnung der Lichtintensität zur Größe der beobachteten Partikel eindeutig möglich ist. Dieser Vorteil wird jedoch mit dem Nachteil des abnehmenden Zählwirkungsgrades für Partikel $<1 \mu \mathrm{m}$ Durchmesser erkauft.

Die Anzahl der pro Zeiteinheit detektierten Streulichtimpulse ist proportional zur Anzahldichte der Partikel in der Luft. Die zeitliche Zuordnung eines Partikelereignisses im Messvolumen kann mit bis zu 5 ms Genauigkeit registriert werden. Die Kalibrierung des OPC erfolgt werkseitig mit Latex-Partikeln, so dass das Gerät bei Messungen an natürlichen Aerosolpartikeln einen auf die optischen Eigenschaften von Latex bezogenen Streulichtäquivalentdurchmesser 
bestimmt. Der Partikelzähler wird mit einer Wasserkühlung betrieben, die Kontrolle des Messvorgangs obliegt einem mitgelieferten, elektronischen Steuergerät. Mit $350 \mathrm{~W}$ ist die Leistungsaufnahme für ein meteorologischen Feldgerät relativ hoch.
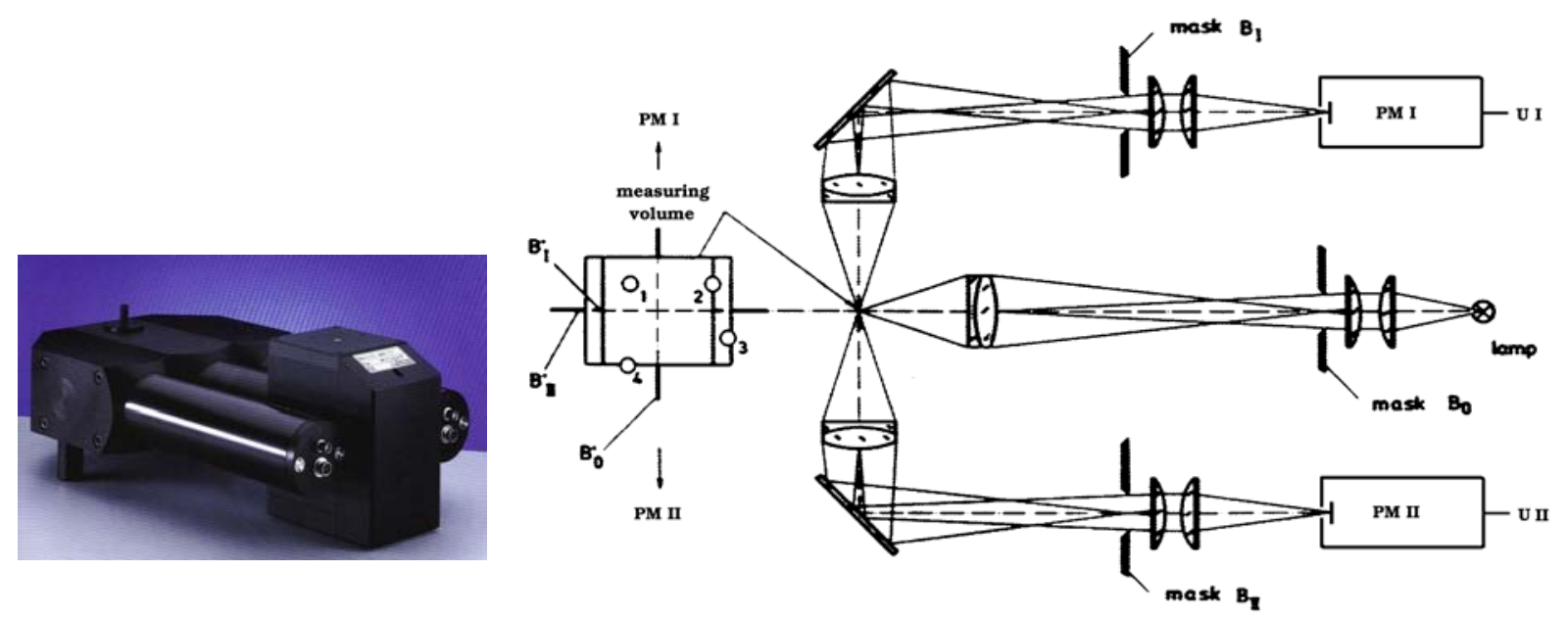

Abb. 10: Aerosolspektrometer PCS-2000 der Firma Palas (links) und schematische Darstellung des Messgerätes (rechts; die Detailsicht zeigt das optisch abgegrenzte Messvolumen mit möglichen Positionen eines sich durch das Messvolumen hindurch bewegenden Partikels ${ }^{12}$ ). Foto/Abbildung: Palas

In der Originalkonfiguration ist der OPC für Partikelkonzentrationen von bis zu $10^{5}$ Teilchen pro $\mathrm{cm}^{3}$ konzipiert. Es wird ein Messbereich von 0,25 bis $17,5 \mu \mathrm{m}$ oder alternativ von 0,6 bis $44 \mu \mathrm{m}$ Partikeldurchmesser abgedeckt, das Messvolumen hat eine Größe von $130 \mathrm{x} 90 \mathrm{x}$ $90 \mu \mathrm{m}$. Der Volumenstrom durch den OPC wird vom Hersteller mit 0,5 bis $2,8 \mathrm{l} / \mathrm{min}$ angegeben. Von dem angesaugten Luftvolumen wird jedoch nur ein Bruchteil (kleiner $1 \%$ ) auf das Vorhandensein von Partikel untersucht, die restliche Luft passiert den OPC ohne das optisch abgegrenzte Messvolumen zu erreichen.

Im Hinblick auf die relativ niedrigen Partikelkonzentrationen am Untersuchungsort wurde der Sensor für die vorliegende Arbeit im Anschluss an eine erste Testmessung im Dezember 1999 durch die Herstellerfirma leicht modifiziert. In dieser modifizierten Version ist es möglich, Partikel bis zu einem Durchmesser von $0,18 \mu \mathrm{m}$ zu detektieren. Gleichzeitig wurde das Messvolumen gegenüber dem Standardmodell vergrößert, und der Volumenstrom auf 5 bis 8 Liter erhöht.

USAT 3/1 und OPC wurden zusammen in $39 \mathrm{~m}$ Höhe über dem Erdboden auf dem Messturm der F1-Fläche installiert. Der Partikelmesser wurde durch eine Metallkiste vor Witterungseinflüssen geschützt, in welcher auch das Steuergerät und ein Messcomputer Platz fanden. Pumpe und Wasserkühlung konnten auf der gleichen Plattform untergebracht werden. Für die quasi-isokinetische Ansaugung der Partikel wurde eine Vorrichtung konstruiert, die durch eine Windfahne ständig in die herrschende Windrichtung gedreht wird (Abb. 11).

\footnotetext{
${ }^{12}$ Es ist obliegt dem Anwender dafür zu sorgen, dass sich zu einem Zeitpunkt nie mehr als ein Partikel gleichzeitig im optischen Messvolumen des OPC befindet.
} 
Abb. 11: Ansaugvorrichtung und

Ultraschallanemometer USAT 3/1 in $39 \mathrm{~m}$ auf der F1-Fläche im Solling

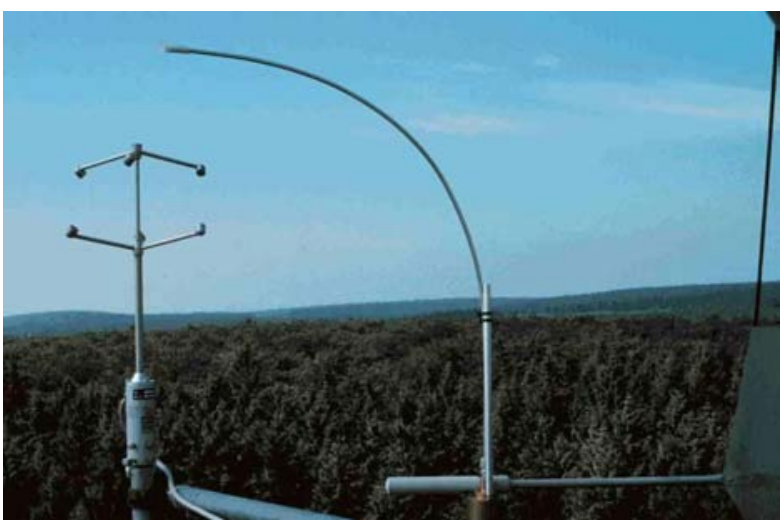

Die Transportstrecke zwischen Ansaugeinrichtung und OPC wurde mit Edelstahlrohr (Durchmesser: $6 \mathrm{~mm}$ ) überbrückt, da hier die geringsten Partikelverluste zu erwarten sind. Die Länge dieser Strecke betrug 1,5 m, die Durchsatzrate etwa 5 Liter pro Sekunde. Damit entsprach die Ansauggeschwindigkeit im Rohr mit knapp $3 \mathrm{~m} / \mathrm{s}$ der mittleren horizontalen Windgeschwindigkeit in der Messhöhe von 39 m. Nachdem die Luft den Partikelzähler passiert hat, wurde sie zu einem Feuchte-, zu einem Druck- und zu einem Massenflussmesser geleitet. Während Niederschlagsereignissen wurde der Ansaugvorgang hauptsächlich zum Schutz der nachgeschalteten Sensoren durch einen Regensensor automatisch unterbrochen (Abb. 12).

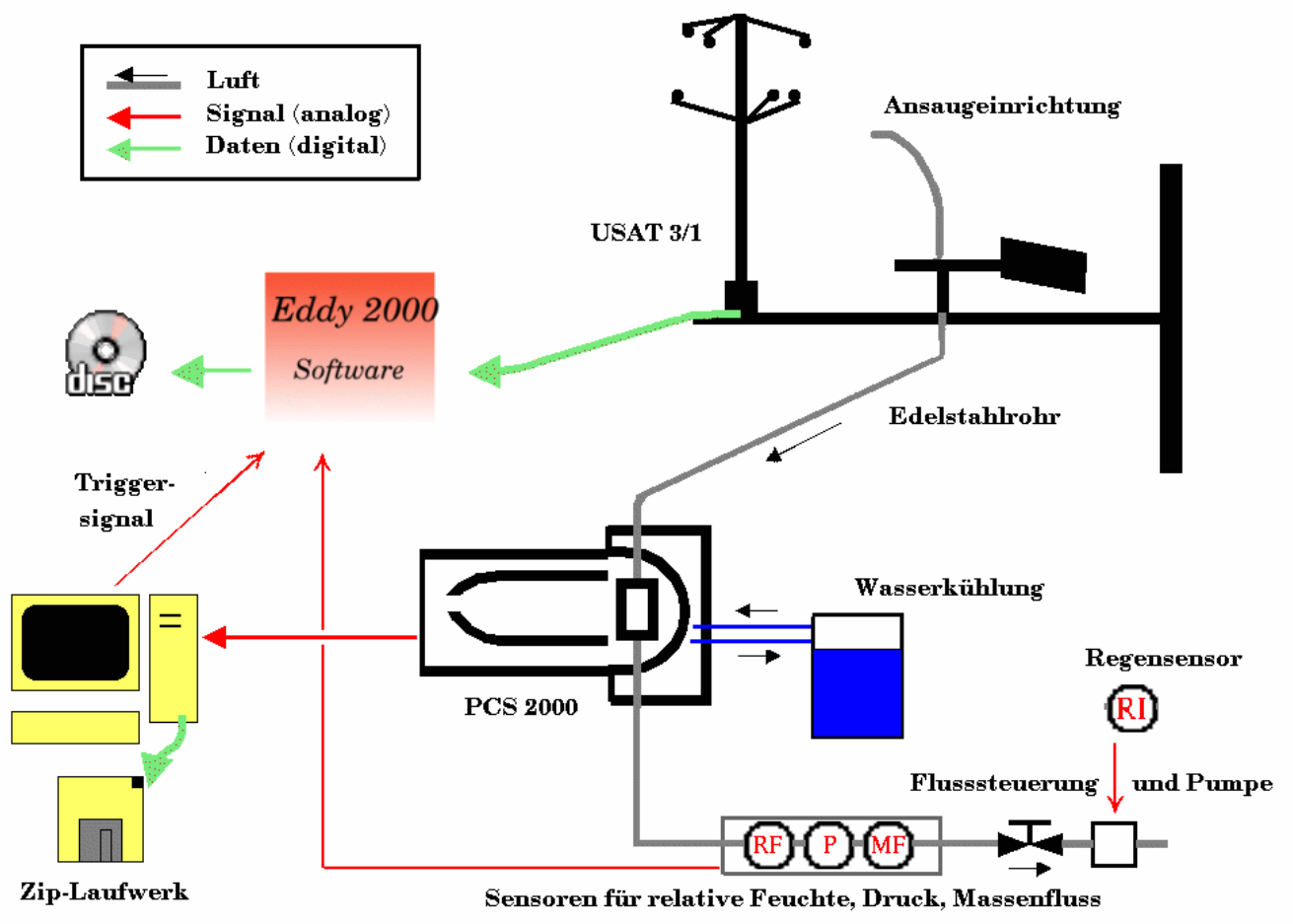

Abb. 12: Schema der Messanlage mit Sonic, Ansaugeinrichtung, OPC und nachgeschalteten Sensoren 
Das USAT 3/1 gehört mit seinem radial-symmetrischen Sondenkopf zu denjenigen Geräten, die für alle Anströmrichtungen eine lokale Beeinträchtigung des Turbulenzfeldes verursachen. Deshalb wurde das Gerät vor Beginn der Messungen einer Windkanaluntersuchung unterzogen, die in Zusammenarbeit mit der Universität Basel im Windkanal der ETH Zürich stattfand. Die Ergebnisse dieser Untersuchungen sind im Anhang dokumentiert. Eine weitere Wartung des Geräts während der Messungen fand nicht statt. Die Kalibrierung des OPC wurde wöchentlich mit Testaerosol getestet. Bei Bedarf wurde das Gerät vor Ort neu kalibriert. Die eingebaute Xenon-Hochdrucklampe musste halbjährlich ausgetauscht werden, dabei wurde der Sensor gleichzeitig gereinigt.

\subsubsection{Datenaufnahme und Datenanalyse}

Die Datenaufnahme erfolgte mit zwei getrennten Erfassungssystemen. Für den OPC ist der Benutzer auf ein durch die Herstellerfirma zu Verfügung gestelltes DOS-Programm angewiesen. Da das Betriebssystem DOS grundsätzlich keinen Mehrprogramm-Betrieb vorsieht, musste so für den Partikelzähler ein separater Messrechner zu Verfügung gestellt werden. Dieser greift hochfrequente Analog-Signale vom Steuergerät des OPC ab, die über eine A/D-Karte erfasst werden. Diese Konfiguration hat zur Folge, dass die Entfernung zwischen Rechner und Steuergerät auf wenige Meter beschränkt werden muss.

Die Daten des Ultraschallanemometers wurden parallel dazu von einem zweiten Rechner aufgezeichnet, der sich in der Messhütte am Fuße des Turmes befand. Auf Grund von Schwierigkeiten mit der vorhandenen Software wurde hierzu ein neues Datenerfassungsprogramm ("Eddy2000") entwickelt. Dieses zweite System wurde mit dem freien Betriebssystem Linux betrieben. Dadurch war jederzeit eine intensive Beobachtung und Steuerung der Messungen über die vorhandene Telefonleitung zur F1-Fläche möglich (Abb. 13).

Abb. 13: Steuerung des Eddy-

Programms vom einem beliebigen, an das Internet angeschlossenen Rechner. Über die Telefonleitung zum Messstandort waren die Messungen jederzeit kontrollierbar.

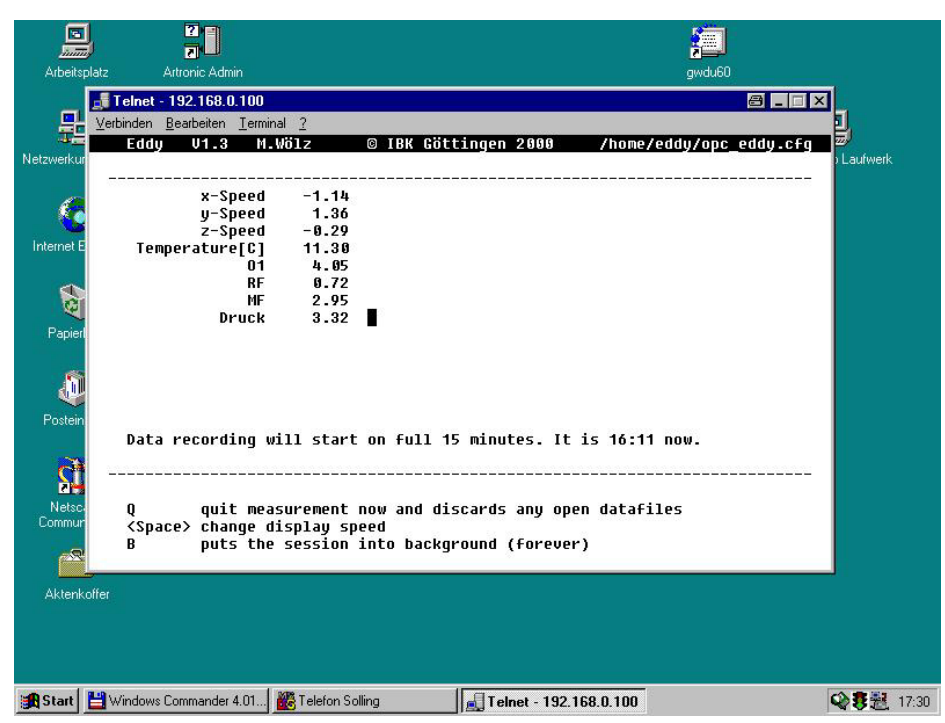


Die Datenaufnahme erfolgte mit $1 \mathrm{~Hz}$ (OPC-Daten) $)^{13}$ bzw. $10 \mathrm{~Hz}$ (Sonic-Daten). Um das Zusammenfügen der aufgenommenen Daten zu erleichtern, wurde durch das OPC System ein Triggersignal erzeugt, welches vom Eddy2000-System registriert wurde. Die aufgenommenen Rohdaten wurden für die spätere Analyse auf CD gebrannt bzw. auf Zip-Medien gesichert. Eine Manipulation der Daten am Messort selbst fand in keiner Weise statt.

Die Datenanalyse erfolgte in drei Schritten. Zunächst wurden die OPC-Daten für die Auswertung vorbehandelt. Dabei wurden aus den Einzel-Partikel-Beobachtungen, die in den Rohdaten für jedes Teilchen noch separat vermerkt sind, Summenwerte abgeleitet. Die 128 Klassen wurden zu 12 logarithmisch-äquidistanten Klassen zusammengefasst (Tabelle 6), wobei die regelmäßig durchgeführten Kalibrationsmessungen berücksichtigt wurden und die sogenannte Randzonenkorrektur durchgeführt wurde. Mit dieser Korrektur werden Partikel von einer weiteren Analyse ausgeschlossen, wenn sie das optische Messvolumen des OPCs nur tangieren, nicht jedoch vollständig erreichen (vgl. Beispielpartikel 2 in Abb. 10, rechtes Bild).

Anschließend wurden die OPC - Daten und die auf $1 \mathrm{~Hz}$ gemittelten Daten des Ultraschallanemometers zusammengefügt. Unter Verwendung der Softwarepakete IDL und ANA wurden die Partikelflüsse sowie interessierende mikrometeorologische Parameter berechnet (Monin-Obuchov-Mischungslänge, Schubspannungsgeschwindigkeit etc.), wobei die in Kapitel 2.2 vorgestellten Formeln und Korrekturen Verwendung fanden.

Tabelle 6: Partikelklassen für die Auswertung nach der Eddy - Kovarianzmethode

\begin{tabular}{|c|c|c|c|}
\hline Klasse & $\begin{array}{c}\text { minimaler } \\
\text { Durchmesser } \\
(\mu \mathrm{m})\end{array}$ & $\begin{array}{c}\text { maximaler } \\
\text { Durchmesser } \\
(\mu \mathrm{m})\end{array}$ & $\begin{array}{c}\text { mittlerer } \\
\text { Durchmesser } \\
(\mu \mathrm{m})\end{array}$ \\
\hline \hline 1 & 0,18 & 0,25 & 0,22 \\
\hline 2 & 0,25 & 0,35 & 0,30 \\
\hline 3 & 0,35 & 0,49 & 0,42 \\
\hline 4 & 0,49 & 0,69 & 0,59 \\
\hline 5 & 0,69 & 0,96 & 0,82 \\
\hline 6 & 0,96 & 1,35 & 1,14 \\
\hline 7 & 1,35 & 1,89 & 1,60 \\
\hline 8 & 1,89 & 2,63 & 2,23 \\
\hline 9 & 2,63 & 3,68 & 3,11 \\
\hline 10 & 3,68 & 5,13 & 4,34 \\
\hline 11 & 5,13 & 7,16 & 6,06 \\
\hline 12 & 7,16 & 10,00 & 8,46 \\
\hline
\end{tabular}

\footnotetext{
${ }^{13}$ Eine Messfrequenz von 1 Herz bedeutet hier lediglich, dass im Sekundenabstand jeweils eine Zeitmarke in die Rohdaten geschrieben wird. In jedem Fall werden alle detektierten Partikel einzeln in den Datenfiles notiert.
} 
Eigene Messungen: Messgelände und Setup

\subsubsection{CPC 3022}

Von Beginn der Messungen an ist bekannt gewesen, dass der erworbene OPC nur einen kleinen Teil des Größenspektrums atmosphärischer Partikel abdecken kann. Es ist deshalb im Rahmen dieser Arbeit versucht worden, ein weiteres Partikelmessgerät in die Eddy Kovarianzmessungen zu integrieren. Hierfür stand aus Institutsbeständen ein Kondensationskernzähler CPC 3022 (hiernach: CPC) der Firma TSI zur Verfügung. Im Gegensatz zum OPC ist der CPC in der Lage, auch noch Aitkenkerne bis zu einem minimalen Radius von $0,01 \mu \mathrm{m}$ zu erfassen. Dies ist möglich, indem die Partikel in übersättigter Umgebung zu einem starken Anwachsen gezwungen werden, so dass sie anschließend gut mit einem Laser detektiert werden können. Die messbare Partikelkonzentration beträgt 0,01 bis $10^{7}$ Partikel pro $\mathrm{cm}^{3}$.

Mit dem vom CPC genutzten Verfahren ist es jedoch nicht möglich, gleichzeitig auch die Größe der einzelnen Partikel zu erfassen und damit die Anzahlverteilung der Partikel als Funktion der Größe zu bestimmen, wie dies beim OPC der Fall ist. Die ermittelte Konzentration bezieht sich folglich auf einen recht breiten Größenbereich, wobei bei Untersuchungen in der Atmosphäre - wie der vorliegenden Arbeit - Partikel mit einem Durchmesser von 0,01 bis $0,1 \mu \mathrm{m}$ den stärksten Anteil an der Gesamtanzahl der gezählten Teilchen haben.

Abb. 14: Mit dem CPC TSI 3022 in Kombination mit einem Größen-Fraktionierer TSI 3071 bestimmtes Partikelspektrum und theoretische Kurve der Größenspektrums nach JUNGE (1963)

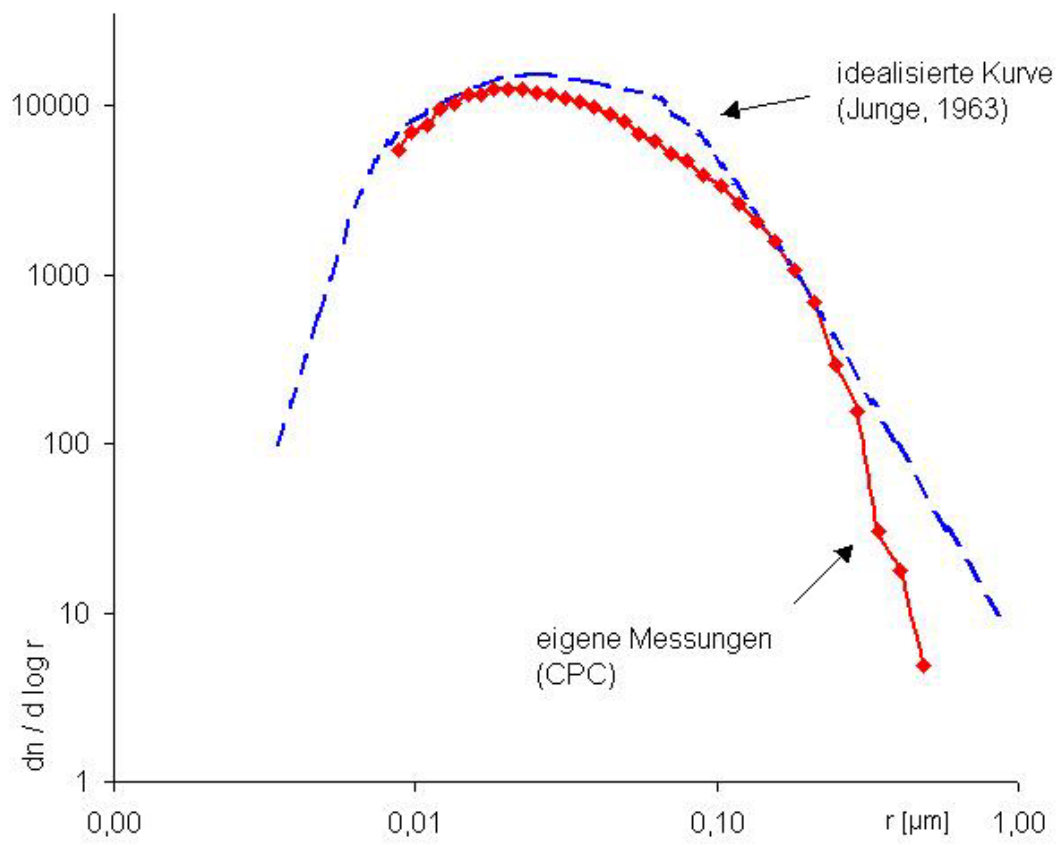

Diesem Manko kann begegnet werden, indem der CPC zusammen mit einem elektrostatischen Größen-Fraktionierer (electrostatic classifier, nach LIU \& PUI, 1974) betrieben wird. Hierbei werden die in der Probenluft vorhanden Partikel zunächst elektrisch aufgeladen und dann durch ein elektromagnetisches Feld bewegt. In Abhängigkeit von der Feldstärke werden auf diese Weise jeweils Partikel eines bestimmten, sehr schmalen Größenbereichs 
extrahiert und anschließend zum CPC geleitet. Mit einer kontinuierlichen Änderung der Feldstärke ist es so möglich, nach und nach das Partikelspektrum zu bestimmen (Abb. 14). Die Prozedur benötigt für das den gesamten bestimmbaren Größenbereich jedoch einen Zeitrahmen von insgesamt 20 bis 40 Minuten. Aus diesem Grunde können mit diesem Verfahren keine Eddy -Kovarianzuntersuchungen durchgeführt werden.

Für die vorliegende Arbeit wurde der CPC gemeinsam mit einem zweiten USAT 3/1 auf $45 \mathrm{~m}$ Höhe des Messturms installiert. Das Gerät wurde zunächst einige Zeit lang ohne einen Größen-Fraktionierer betrieben, um die Möglichkeiten einer Eddy - Kovarianzmessung mit dem CPC generell austesten zu können. Anschließend wurde ein Größen-Fraktionierer TSI 3071 mit in die Messungen einbezogen. Die Feldstärke im Fraktionierer wurde jedoch für jeweils mehrere Tage konstant gehalten, so dass also nur die Konzentration einer einzigen, ausgewählten Größenklasse bestimmt wurde. Während beim Betrieb des CPC ohne GrößenFraktionierer durchaus brauchbare Ergebnisse erzielt werden konnten, hat sich für das Gesamtsystem dagegen herausgestellt, dass dieses nicht für Eddy - Kovarianzmessungen geeignet ist. 


\section{Resultate}

In diesem Kapitel werden die Messresultate der eigenen Untersuchungen beschrieben. Im Mittelpunkt stehen dabei die Messungen mit dem optischen Einzelpartikelzähler PCS-2000 (hiernach: OPC). Alle Größenangaben beziehen sich - wenn nicht anders vermerkt - auf den Partikeldurchmesser und sind in Mikrometer angegeben. Die Partikelklassen entsprechen den Größenangaben in Tabelle 6. Die Bezeichnung "alle Partikel" wird verwendet, wenn die Konzentration über alle 12 Partikelklassen integriert wurde.

\subsection{Messungen mit dem OPC}

\subsubsection{Mittlere Partikelkonzentrationen im Solling}

Die mittlere Partikelkonzentration in dem durch den OPC abgedeckten Größenbereich von etwa 0,2 bis $10 \mu \mathrm{m}$ Durchmesser beträgt für den betrachteten Zeitraum knapp 800 Partikel / $\mathrm{cm}^{3}$. Die Konzentration schwankt jedoch stark, so dass auch zahlreiche Ereignisse mit mehreren tausend Partikeln pro $\mathrm{cm}^{3}$ beobachtet werden konnten. Insbesondere sind die Partikelkonzentrationen starken Veränderungen unterworfen, wenn die relative Luftfeuchte auf über $98 \%$ ansteigt. Dies wird im nächsten Abschnitt gezeigt. Bei den folgenden Betrachtungen sollen solche Situationen zunächst von einer Analyse ausgeschlossen werden.

Der mittlere Tagesgang der Partikelkonzentration für den gesamten Messzeitraum ist in allen Größenklassen durch ein Konzentrationsminimum am frühen Nachmittag und ein Maximum am frühen Morgen gekennzeichnet (Abb. 15).

Abb. 15: Mittlere Tagesgänge der Konzentration der fünf kleinsten Partikelklassen sowie der relativen Feuchte (grau unterlegt). Berücksichtigt wurden alle Halbstundenereignisse mit einer mittleren relativen Luftfeuchte $<95 \%$ im Zeitraum vom 2.2.2000 bis zum 31.8.2001

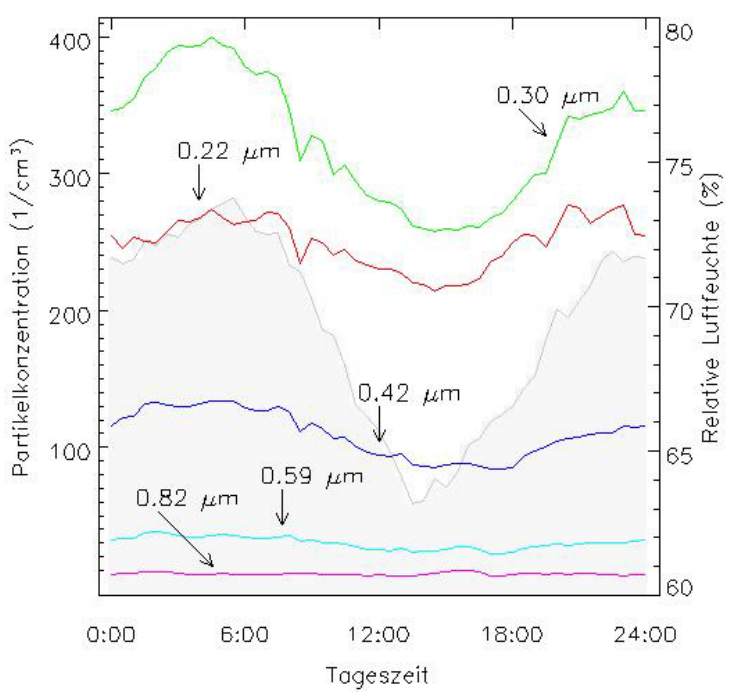


Betrachtet man die konkreten Partikelkonzentrationen an einzelnen Messtagen, so ist die beschriebene Regelmäßigkeit des mittleren Ganges (Minimum am Nachmittag, Maximum am Morgen) jedoch nur selten in dieser einfachen Form wiederzuerkennen. Zwar ist das Konzentrationsminimum auch an den einzelnen Messtagen fast immer am frühen Nachmittag zu finden, ansonsten sind die einzelnen Tagesverläufe aber durch verschiedenenartige Trends charakterisiert. So steigt die Konzentration im Verlaufe des Vormittags des 5. Mai beispielsweise noch fast bis 12 Uhr merklich an, während sie am Folgetag im gleichen Zeitraum deutlich absinkt (Abb. 16).

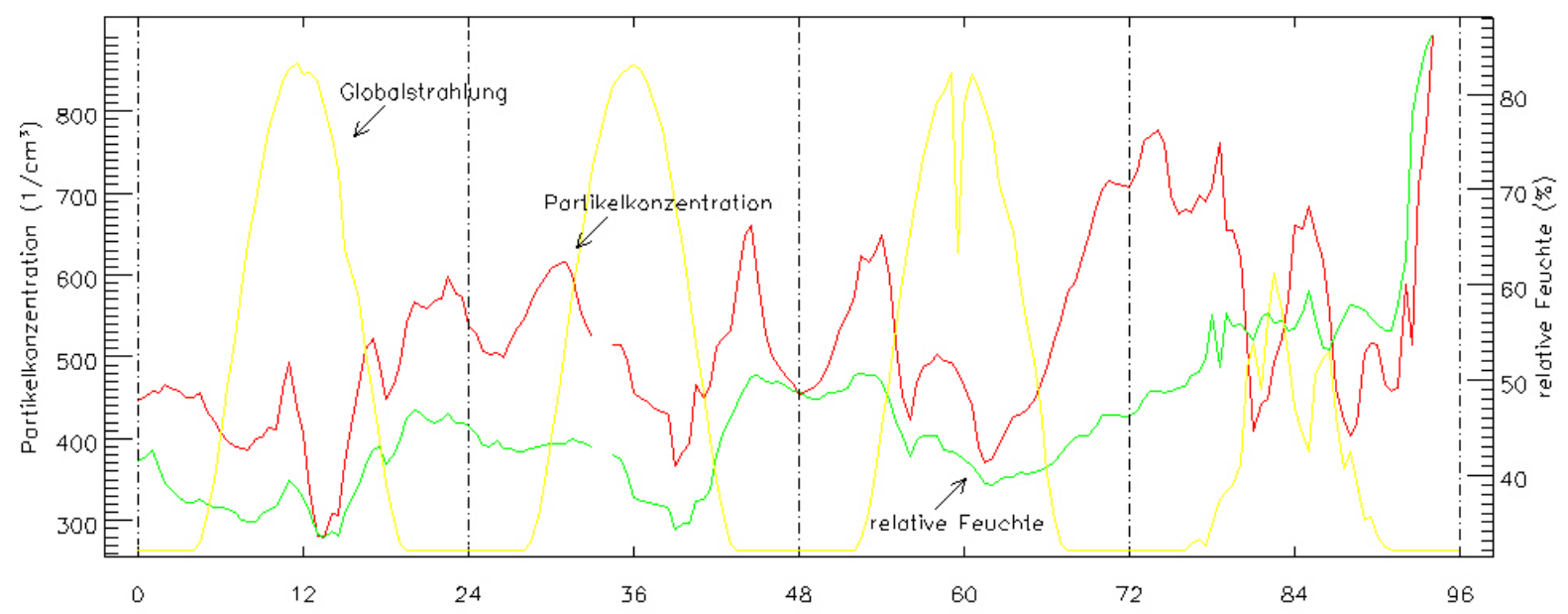

Abb. 16: Partikelkonzentration (OPC, alle Klassen - rot), relative Luftfeuchte (grün) und Globalstrahlung (gelb, ohne Skala) für den 5. bis 8. Mai 2000. x-Achse: Stunden nach dem 5.5.00, 0:00 Uhr

Die Abnahme der Konzentration vom Morgen bis zum frühen Nachmittag und die darauf folgende Zunahme während der Nacht im mittleren Tagesverlauf können theoretisch auf drei verschiedene Phänomene zurückgeführt werden:

1. die Änderung der Höhe und damit des Volumens der Planetarischen Grenzschicht (PBL) im Tagesverlauf (Verdünnungseffekt),

2. eine Abhängigkeit der Partikelkonzentration von der relativen Feuchte, die einen sehr ähnlichen mittleren Tagesgang aufweist wie die Partikelkonzentration, und/oder

3. den tagsüber verstärkten Vertikalaustausch zwischen Erdoberfläche und Atmosphäre.

Von diesen drei Erklärungsvarianten kann die erste Variante wahrscheinlich weitestgehend ausgeschlossen werden. Wäre der Gang der Partikelkonzentration nämlich hauptsächlich von der Höhe der PBL beeinflusst, müsste die Partikelkonzentration als Folge des Verdünnungseffektes insbesondere an Tagen mit einem stark ausgeprägten Tagesgang der Globalstrahlung im Tagesverlauf deutlich absinken. Dies ist jedoch z.B. für den 5. Mai nicht festzustellen. Dagegen fällt auf, dass der Konzentrationsverlauf an den betrachteten Einzeltagen tatsächlich weitgehend dem Verlauf der relativen Feuchte folgt. Die Gründe für eine solche Abhängigkeit 
können in Prozessen der Partikelproduktion und -umwandlung in der Atmosphäre vermutet werden. Diese Vorgänge sind noch immer Inhalt aktueller Forschung (z.B. MÄKELÄ ET AL., 1997) und nicht im Detail verstanden. Alternativ könnte die Erklärung des Phänomens darin bestehen, dass zu unterschiedlichen Zeiten Luftpakete aus unterschiedlichen Orten der Atmosphäre zum Untersuchungsort gelangen, welche dann für den jeweiligen Ursprungsort typische Verhältnisse von Partikelkonzentration und Luftfeuchte aufweisen. Als unwahrscheinlich kann es dagegen angesehen werden, dass die Änderung der Partikelkonzentration die Folge feuchtebedingten Wachstums beispielsweise von Teilchen von ursprünglich $<0,2 \mu \mathrm{m}$ Durchmesser ist, da die relativen Feuchten für die betrachteten Tage mit 40 bis $60 \%$ eher niedrig sind. Der Einfluss des Tagesganges der vertikalen Deposition (Erklärungsvariante 3) auf den Gang der Partikelkonzentration liegt auf der Hand und kann wohl in jedem Fall als gegeben angenommen werden.

Ein Zusammenhang zwischen der Herkunft der Luftmassen und der Partikelkonzentration kann nicht eindeutig gezeigt werden. Es fällt aber auf, dass niedrige Partikelkonzentrationen insbesondere bei Südwestwindlagen auftreten. Höhere Konzentrationen finden sich dagegen bei allen Wetterlagen. Dies wird in Abb. 17 verdeutlicht. Hier werden Rückwärtstrajektorien für den untersuchten Standort dargestellt. Die Trajektorien wurden nach der Partikelkonzentration zum Zeitpunkt des Eintreffens der Luftmasse am Untersuchungsort in Tage mit Konzentrationen $<500$ Partikeln pro $\mathrm{cm}^{3}$ (Abb. 17, links) und Tage mit Konzentrationen $>900$ Partikeln pro $\mathrm{cm}^{3}$ (Abb. 17, rechts) eingeteilt. Wiederum wurden Beobachtungen, bei denen die Luftfeuchtigkeit bei Ankunft der Luftmasse größer $>95$ \% war, nicht mit in die Betrachtung einbezogen, da hier die Partikelkonzentrationen kurzzeitig stark modifiziert sein können.
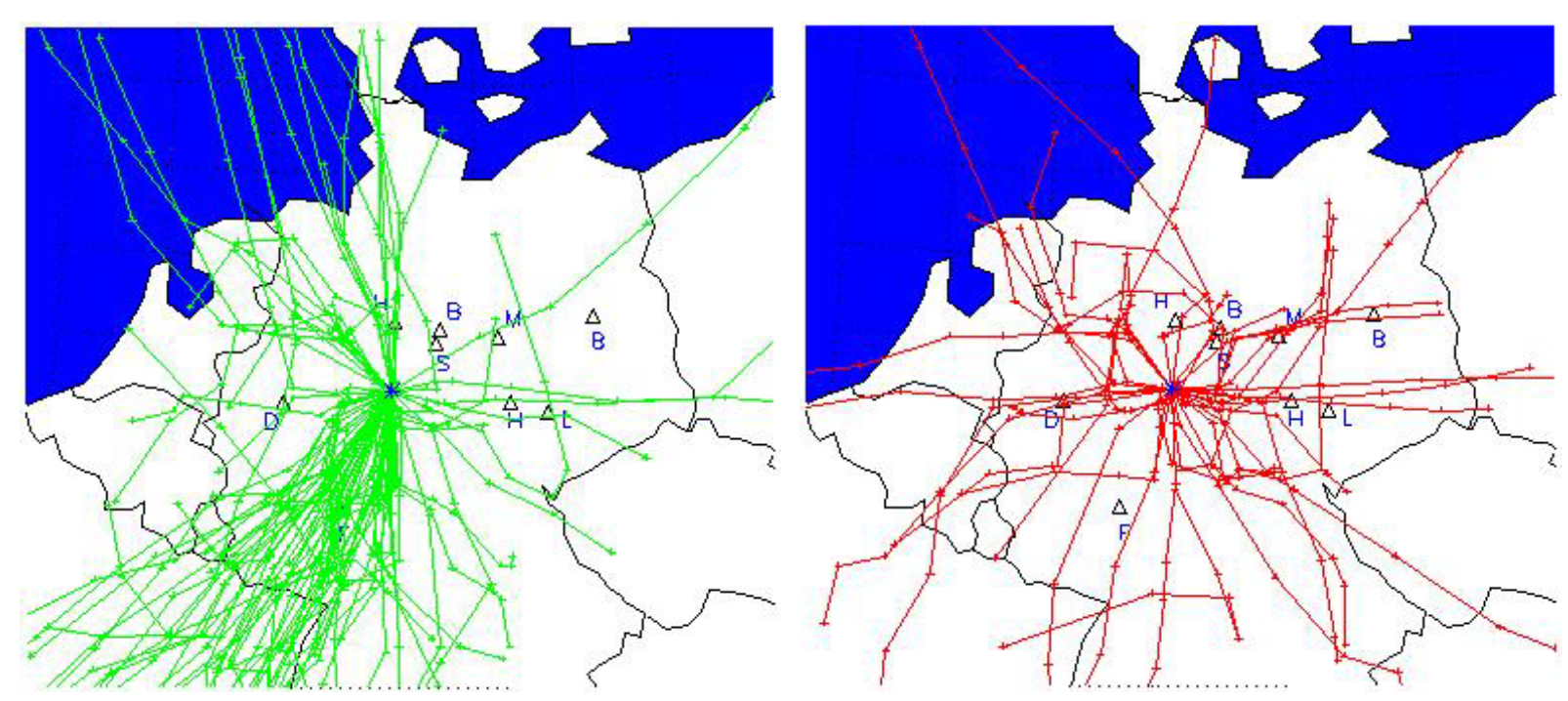

Abb. 17: Tägliche Rückwärtstrajektorien für den Solling. Ankunftszeit jeweils 17:00 Uhr, Laufzeit 36 Stunden; links: für Partikelkonzentrationen jeweils zum Zeitpunkt der Ankunft im Solling $<500$ Partikeln $/ \mathrm{cm}^{3}$; rechts: > 900 Partikeln $/ \mathrm{cm}^{3}$ (alle Klassen des OPC). Markiert sind die Städte Berlin, Magdeburg, Braunschweig, Salzgitter, Hannover, Leipzig, Halle, Frankfurt a.M. und Dortmund. 
Resultate der eigenen Messungen

\subsubsection{Partikelspektrum}

Die meisten Partikel werden vom OPC in der Größenklasse zwei (mittlerer Durchmesser: $0,30 \mu \mathrm{m})$ gezählt. Mit 321 Partikeln pro $\mathrm{cm}^{3}$ ist die Anzahldichte hier im zeitlichen Mittel fast dreifach so hoch wie in der nächsthöheren Größenklasse drei (114 Partikel pro $\left.\mathrm{cm}^{3}\right)$ und deutlich größer als in der kleinsten Klasse eins $\left(252\right.$ Partikel pro $\left.\mathrm{cm}^{3}\right)$. Für die folgenden Partikelklassen nehmen die Konzentrationen mit zunehmender Partikelgröße weiter ab, um schließlich in der größten Klasse das Minimum von 0,02 Partikel pro $\mathrm{cm}^{3} \mathrm{zu}$ erreichen (Tabelle 7).

Tabelle 7: Mittlere Konzentrationen für die 12 Partikelklassen des OPC

\begin{tabular}{|c|c|c|c|}
\hline Klasse & $\begin{array}{c}\text { mittlerer } \\
\text { Durchmesser } \\
(\mu \mathrm{m})\end{array}$ & $\begin{array}{c}\text { mittlere } \\
\text { Konzentration } \\
\left(1 / \mathrm{cm}^{3}\right)\end{array}$ & $\begin{array}{c}\text { mittlere } \\
\text { Konzentration } \\
(\mathrm{d} \mathrm{N} / \mathrm{d} \log \mathrm{r})\end{array}$ \\
\hline \hline 1 & 0,22 & 251,6 & 1737 \\
\hline 2 & 0,30 & 320,6 & 2213 \\
\hline 3 & 0,42 & 114,1 & 787,3 \\
\hline 4 & 0,59 & 31,79 & 219,4 \\
\hline 5 & 0,82 & 8,716 & 60,16 \\
\hline 6 & 1,14 & 4,962 & 34,25 \\
\hline 7 & 1,60 & 3,878 & 26,76 \\
\hline 8 & 2,23 & 1,809 & 12,48 \\
\hline 9 & 3,11 & 0,783 & 5,404 \\
\hline 10 & 4,34 & 0,242 & 1,672 \\
\hline 11 & 6,06 & 0,038 & 0,263 \\
\hline 12 & 8,46 & 0,016 & 0,113 \\
\hline
\end{tabular}

Damit suggeriert der OPC für die untersuchte Fläche im Solling ein Konzentrationsmaximum bei einem Partikeldurchmesser von $0,3 \mu \mathrm{m}$. Von dieser Größenklasse aus fällt die Anzahldichte sowohl zu den kleineren und als auch zu den größeren Partikeln hin scheinbar ab (Abb. 24). Diese Aussage steht im Widerspruch sowohl zu der üblicherweise angenommenen Form des Partikelspektrums (z.B. JuNGE, 1963) als auch zu den eigenen Messungen mit dem zweiten Messsystem (Abb. 14). Wie in den genannten Abbildungen zu erkennen ist, wäre das Maximum der Partikelkonzentration eigentlich in der kleinstes Klasse (mittlerer Durchmesser: 0,2 $\mu \mathrm{m}$ ) und nicht in der zweitkleinsten Klasse zu erwarten gewesen. Die Partikelkonzentration würde dann von dieser kleinsten Klasse aus über den gesamten Größenbereich abfallen. Dass dies nicht der Fall ist, kann nur als ein Artefakt des Messsystems in dem Sinne interpretiert werden, dass die Zähleffektivität des OPC in der ersten Größenklasse gegenüber den anderen Klassen signifikant erniedrigt ist. Diese Vermutung ist von der Herstellerfirma auf Anfrage bestätigt worden. Die Grenzen des eingesetzten Partikelzählers für Untersuchungen des atmosphärischen Aerosols werden hier deutlich. 
Abweichend vom bisher gesagtem kommt es im Falle einer Erhöhung der relativen Luftfeuchtigkeit auf mehr als $98 \%$ zu einer zeitweiligen Verschiebung des Konzentrationsmaximums in Richtung größerer Partikelklassen. Die Anzahl der Partikel mit einem Durchmesser von 0,6 bis $4 \mu \mathrm{m}$ erhöht sich dabei signifikant, während gleichzeitig die Zahl der Partikel $<0,5 \mu \mathrm{m}$ netto reduziert wird (Abb. 18). Es kann angenommen werden, dass es sich bei den unter diesen Bedingungen vermehrt auftretenden Teilchen $>0,6 \mu \mathrm{m}$ um Nebeltröpfchen handelt, da deren Größe nach einer Datenzusammenstellung von WARNECK (2000) zwischen 0,5 und $30 \mu \mathrm{m}$ (häufigster Wert: $0,8 \mu \mathrm{m}$ ) anzusetzen ist. Im folgenden sollen diese Zeiträume deshalb als "Nebelereignisse" bezeichnet werden. Der Begriff "Nebel" wird hier verwendet, obwohl noch keine Aussagen zu visuellen Beobachtungen getroffen wurden. Die klassische, meteorologische Definition von Nebel bezieht sich auf diese Beobachtungen $($ Nebel $=$ Sichtweite kleiner $1000 \mathrm{~m})$.

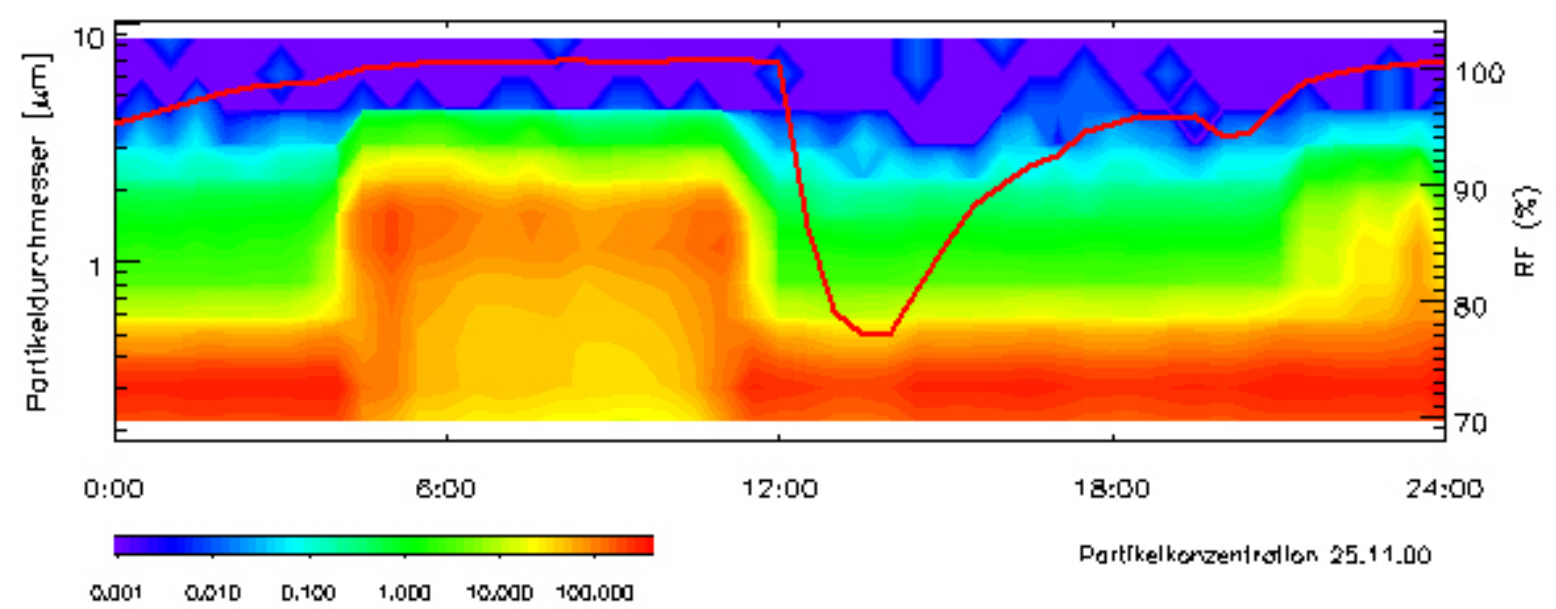

Abb. 18: Verschiebung des Konzentrationsmaximums im Partikelspektrum zu Gunsten größerer Partikel als Folge des Anstiegs der relativen Feuchte auf über $98 \%$ in der Umgebungsluft (durchgezogene rote Linie)

Um die Vermutung zu überprüfen, dass es sich bei den beobachteten Ereignissen tatsächlich um Nebel handelt, wurde eine einfache elektronische Kamera (eine Webcam) installiert, die in regelmäßigen Abständen (alle 10 Minuten) jeweils ein Bild der Umgebung des Messturms auf der F1-Fläche aufnimmt. Mit diesen Bildern lässt sich zeigen, dass es in Zusammenhang mit den beobachteten Ereignissen tatsächlich zu einer starken Einschränkung der Sichtweite in der Atmosphäre kommt, wenn auch die Qualität der Bilder eine stärkere Quantifizierung dieser Aussage nicht erlauben. Als Beleg wird in Abb. 19 ein Tagesausschnitt vom 24. März 2001 dokumentiert. Auf den Bildern der Webcam ist die Grenze zwischen Wald und Himmel nur genau zu den Zeiten eindeutig erkennbar, an denen das Konzentrationsmaximum im Partikelspektrum nicht zu größeren Partikeln hin verschoben ist. Im Gegensatz hierzu kann der Horizont am nebelfreien 30. März des gleichen Jahres ganztägig identifiziert werden (Abb. 20). 

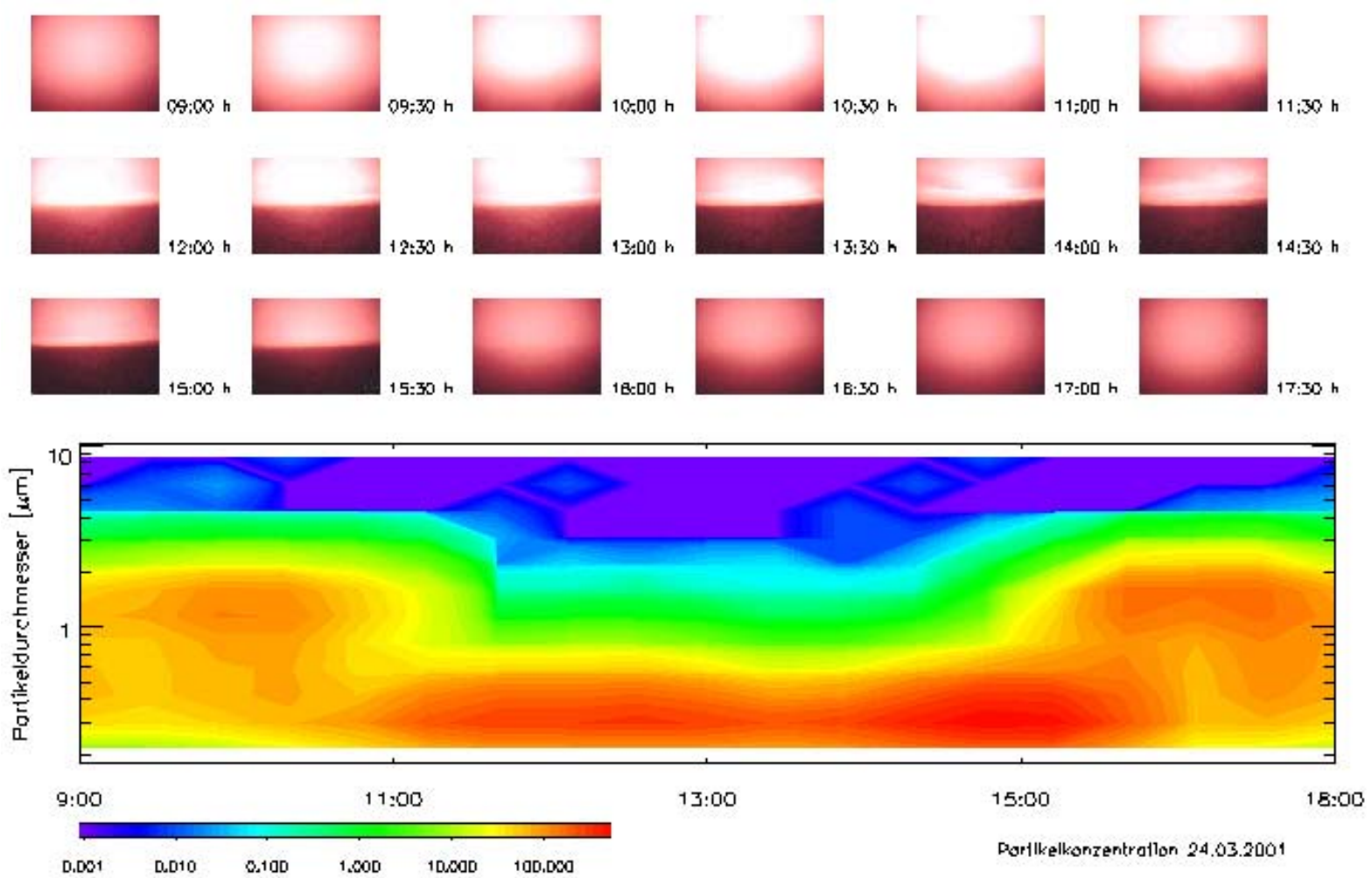

Abb. 19: Webcam - Bilder und Partikelspektrum für den 24. März 2001. 9:00 bis 18:00 Uhr
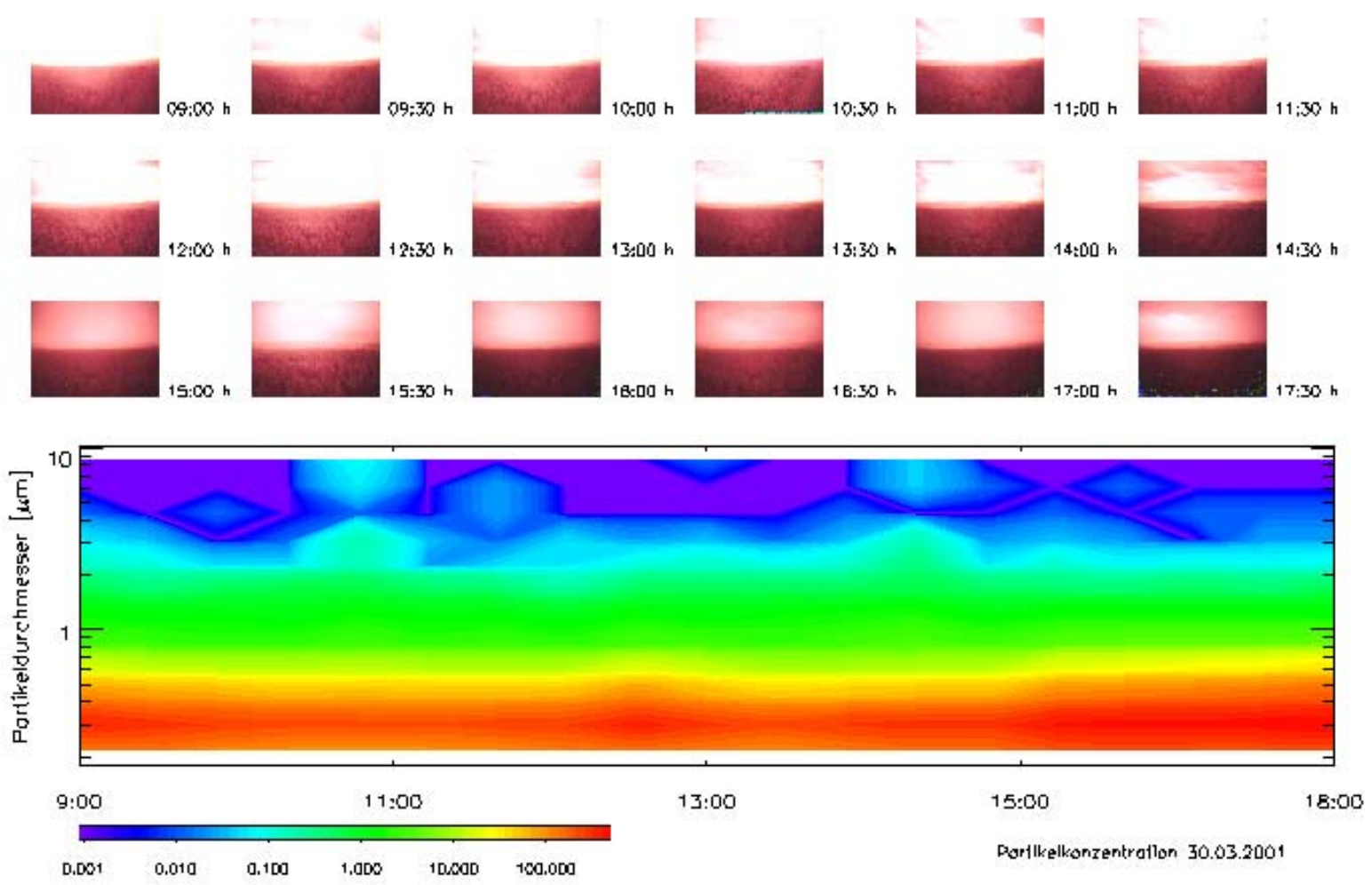

Abb. 20: Webcam - Bilder und Partikelspektrum für den 30. März 2001, 9:00 bis 18:00 Uhr 
Die zeitliche Dauer der beobachteten Nebelereignisse schwankt zwischen wenigen Minuten und 20 Stunden. Wie aus Tabelle 8 hervorgeht, trat der meiste "Nebel" im Untersuchungszeitraum in den Monaten Februar, März und Oktober 2000 sowie März und April 2001 auf. Im Tagesverlauf ist die Wahrscheinlichkeit für das Auftreten von Nebelereignissen in den frühen Morgenstunden zwischen 3:00 und 7:00 Uhr mit knapp 13\% am höchsten, während die Wahrscheinlichkeit eine Nebelereignisses am frühen Nachmittag mit weniger als $4 \%$ dreimal niedriger ist (Abb. 21).

Abb. 21: Nebelwahrscheinlichkeit für den untersuchten Fichtenaltbestand als Funktion der Tageszeit. Nebel ist hier definiert als Zeitraum, in dem für einen Mittelungszeitraum von jeweils $30 \mathrm{Mi}-$ nuten das Maximum der Partikelkonzentration $\mathrm{d} \mathrm{N} / \mathrm{d} \log \mathrm{r}$ in einer Größenklasse mit einem Durchmesser $>0,6 \mu \mathrm{m}$ liegt.

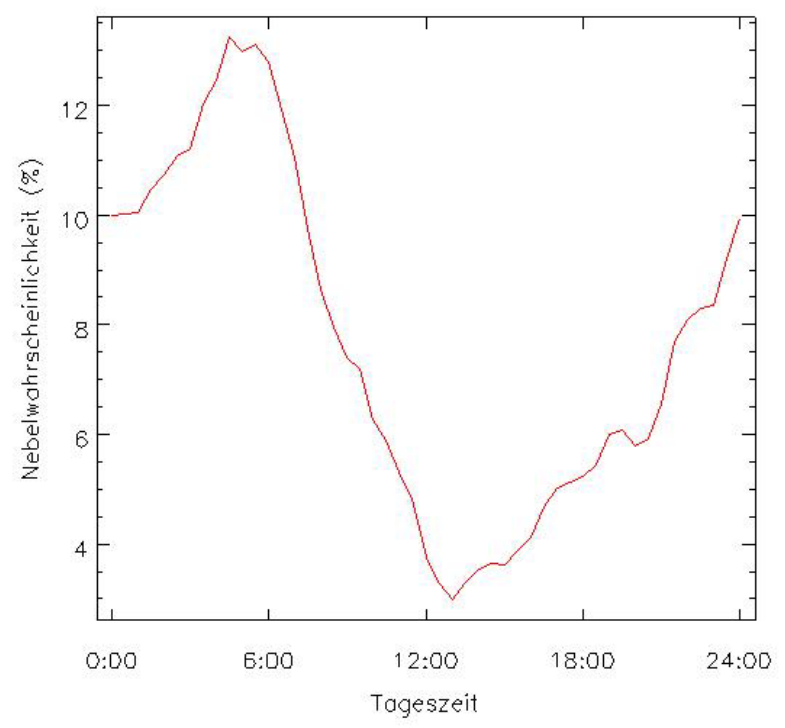

Der Übergang von einer "nebelfreien” Zeit zu einem "Nebelereignis" erfolgt nicht einheitlich. Es werden sowohl sehr rasche als auch langsame Übergänge beobachtet. Bei einem schnellen Übergang zu einem Nebelereignis werden die Partikelkonzentrationen in den Klassen der kleineren Partikel $(0,22$ bis $0,59 \mu \mathrm{m})$ innerhalb von nur einer Minute um bis zu $80 \%$ reduziert. In der gleichen Zeit steigen die Konzentrationen in den größeren Partikelklassen $(0,812$ bis 3,0 $\mu \mathrm{m})$ deutlich an. Partikel der Größenklasse 1,6 $\mu \mathrm{m}$ können dabei den stärksten Konzentrationszuwachs realisieren (Abb. 22). Ein wichtiges Detail dabei dürfte die Beobachtung sein, dass der Anstieg der relativen Feuchte im dargestellten Fall der Veränderung der Partikelkonzentration nicht vorausgeht, sondern vielmehr nachfolgt. Auch steigt der Betrag der Feuchte zunächst nicht auf über $95 \%$ an. Beide Tatsachen können als ein Indiz dafür gedeutet werden, dass in diesem betrachteten Beispiel die Nebeltröpfchen nicht am Untersuchungsort selbst produziert wurden, sondern in Form von aufliegenden Wolken bzw. Wolkenfetzen antransportiert wurden. In der Tat werden aufliegende Wolken für die Hochlagen des Sollings häufig beobachtet.

Ganz anders stellt sich die Situation in Abb. 23 dar. Hier steigt die relative Feuchte über einen Zeitraum von mindestens einer halben Stunde kontinuierlich an (Zeitraum nur unvollständig in der Abbildung dargestellt). Parallel zu diesem Anstieg verringern sich die Konzentrationen in den Klassen der kleineren Partikel $(0,22$ bis $0,42 \mu \mathrm{m})$ ebenfalls kontinuierlich, während 
gleichzeitig die Konzentrationen in den größeren Partikelklassen $(0,59$ bis 3,11 $\mu \mathrm{m})$ zunächst zögerlich, dann deutlicher ansteigen. Bei einer Feuchte von etwa 98 \% kommt schließlich der "Durchbruch": die Konzentration der kleineren Partikel wird kurzzeitig in jeder Klasse auf jeweils 200 Partikel pro $\mathrm{cm}^{3}$ reduziert, während die Konzentrationen der größeren Partikel in einigen Größenklassen auf mehr als 400 Partikel pro $\mathrm{cm}^{3}$ ansteigen. Diese Situation ist jedoch nicht stabil, die Verhältnisse drehen sich zeitweilig wieder um, bis schließlich zwei Minuten später wiederum die kleineren Partikel stark reduziert werden. Diese Vorgänge setzten sich nun über mehrere Minuten und Stunden lang fort. Es kann davon ausgegangen werden, dass in diesem Fall - im Gegensatz zur Situation von Abb. 22 - lokale Prozesse des Feuchtewachstums ursprünglich kleinerer, später dann zeitweilig größerer Teilchen beobachtet wurden.

Abb. 22: "Rasches"

Nebelübergangsereignis (4.8.01, 1:00 Uhr).

Dargestellt sind die

Konzentrationsverläufe der Partikelklassen 0,22 (rot, oben), 0,30 (grün, o.), 0,42 (blau, o.), 0,59 (hellblau, o.), 0,82 (rot, unten), 1,14 (grün, u.), 1,60 (blau, u.), 2,23 (hellblau, u.) und 3,11 (türkis, u.) sowie der Gang der relativen Luftfeuchte (jeweils grau unterlegt) ${ }^{14}$.
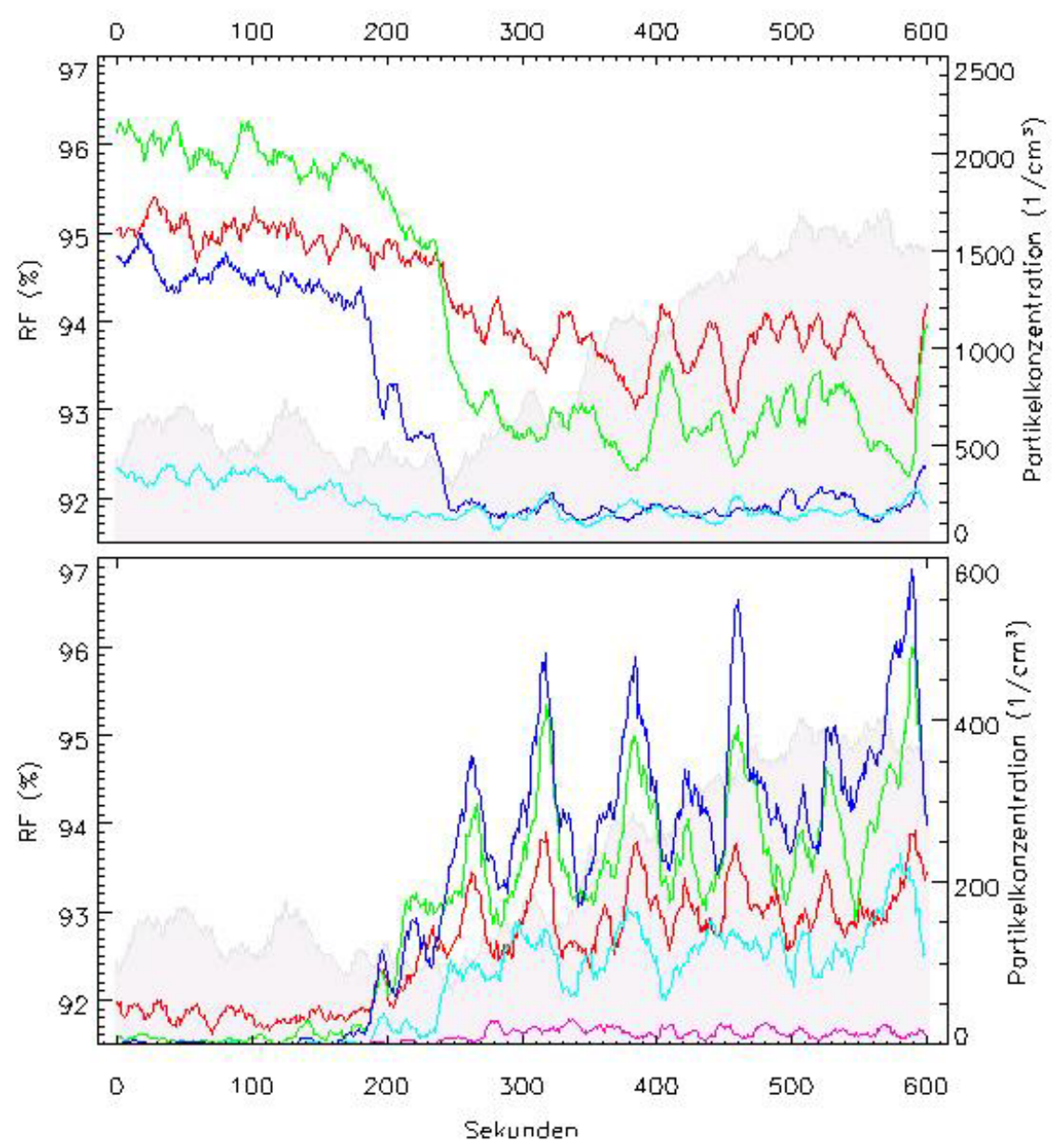

Wie die beiden Beispiele zeigen, kann mit Hilfe des OPCs das Auftreten von Nebelereignissen sowohl quantitativ festgestellt als auch die Art der Entstehung des Ereignisses qualitativ

\footnotetext{
${ }^{14}$ Werden in der vorliegenden Arbeit Verläufe der relativen Feuchte der Umgebungsluft mit einer zeitlichen Auflösung größer als einer halben Stunde gezeigt, handelt es sich um Werte, die aus dem Feuchtesignal innerhalb des Partikelmesssystems zurückgerechnet wurden.
} 
abgeschätzt werden. Da für die vorliegende Arbeit kein Nebelsichtgerät zur Verfügung stand, ist eine noch detailliertere Analyse der Ergebnisse und ein Vergleich mit einem unabhängigen Messgerät nicht möglich.

Abb. 23: "Langsames"

Nebelübergangsereignis (7.3.00, 21:00 Uhr).

Dargestellt sind die

Konzentrationsverläufe der Partikelklassen 0,22 (rot, oben), 0,30 (grün, o.), 0,42 (blau, o.), 0,59 (rot, unten), 0,82 (grün, u.), 1,14 (blau, u.), 1,60 (hellblau, u.), 2,23 (türkis, u.) und 3,11 (gelb, u.) sowie der Gang der relativen Luftfeuchte (jeweils grau unterlegt).

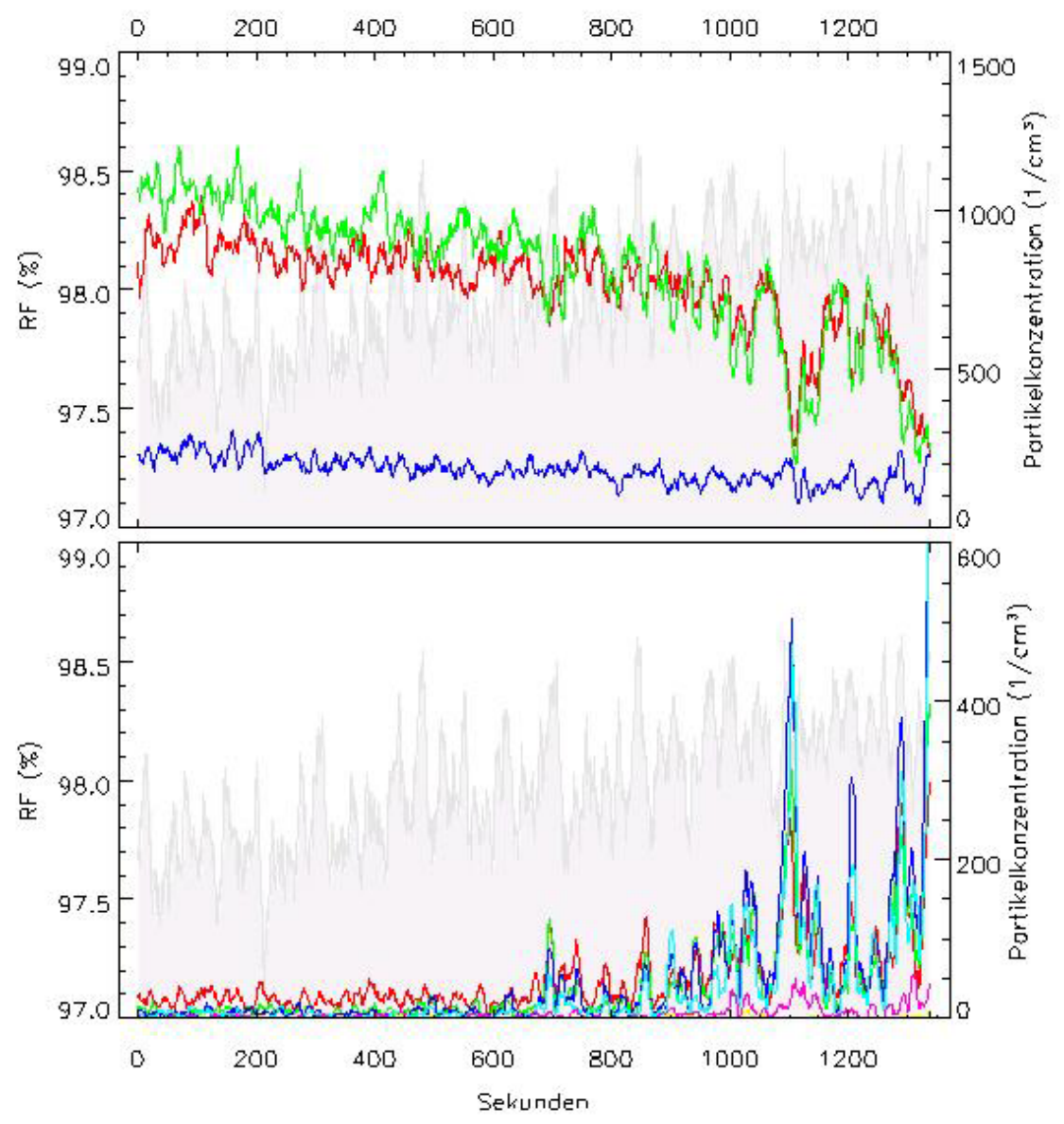

Die feuchtebedingten Verschiebungen des Maximums im Partikelspektrum spiegeln sich auch im mittleren Aerosolspektrum wieder. Dieses ist in Abb. 24 dreimal aufgetragen: (a) für alle 17700 getätigten Beobachtungen, (b) für Zeiten mit relativen Luftfeuchten $<95 \%(9600$ Beobachtungen) und (c) für Zeiten mit relativen Luftfeuchten $>98 \%$ (3400 Beobachtungen). Nur Kurve (b) befindet in guter Übereinstimmung mit der idealisierten Kurvenform von JUNGE (1963). In den beiden anderen Fällen sind abweichend von dieser Idealkurve stark erhöhte Partikelkonzentrationen bei Durchmessern von 0,8 bis $3 \mu \mathrm{m}$ zu erkennen.

Ein von CONSTANTIN (1993) im September 1991 für den gleichen Standort aufgenommenes Partikelspektrum entspricht einer Mischform aus den Kurven (a) und (b). Ein Vergleich mit den Daten von JAENICKE (1993) weist die Aerosolverteilung im Solling als Übergang zwischen einem Reinluftaerosol (charakterisiert durch geringe Gesamtpartikelzahl insbesondere bei Teilchen $<1 \mu \mathrm{m}$ Durchmesser) und einem kontinentalen Aerosol mit höherer Partikelzahl aus. 


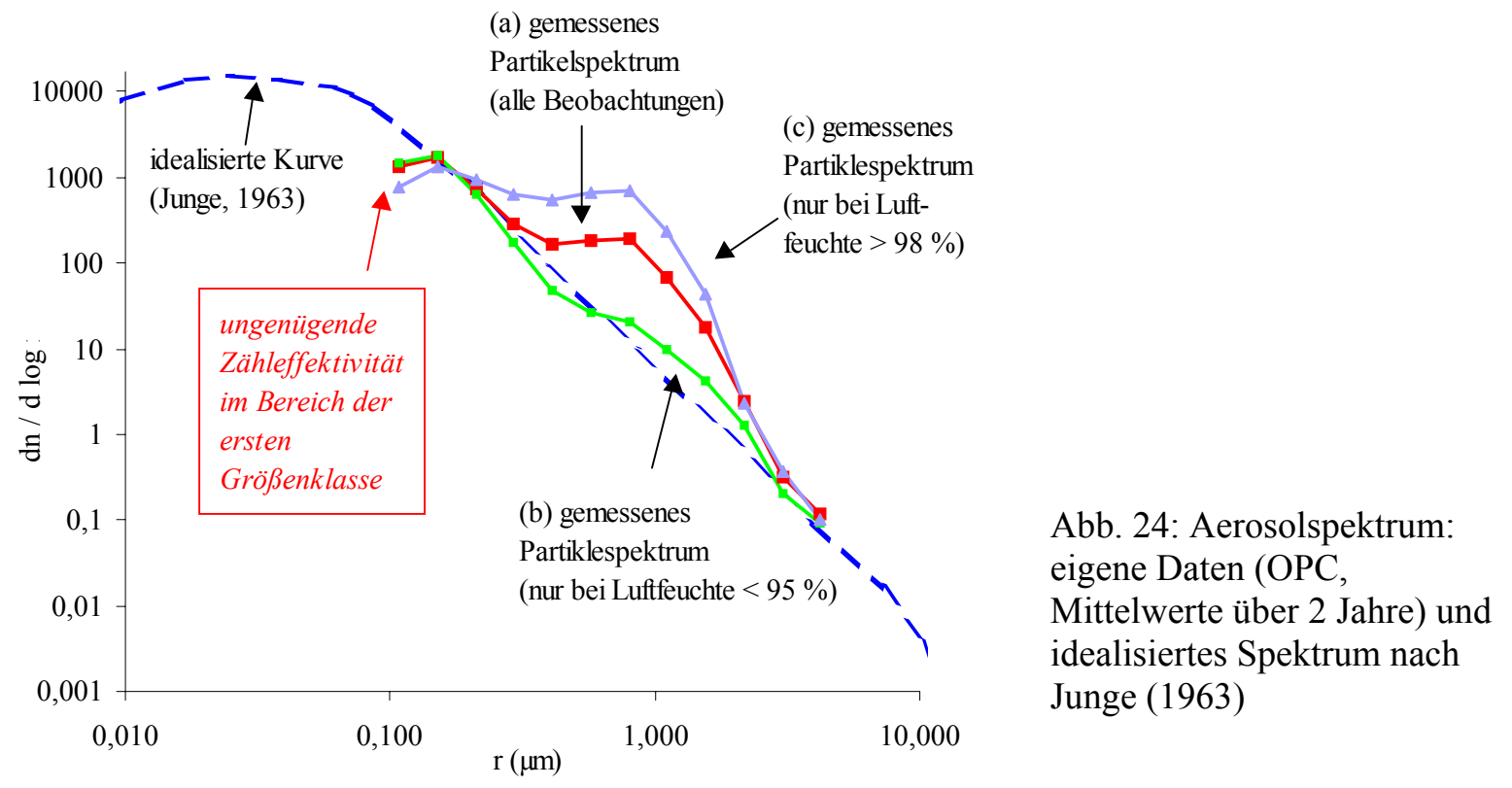

Tabelle 8: Zeiträume mit Nebel für die einzelnen Monate (Angaben in Prozent der theoretisch zur Verfügung stehenden Messzeit bzw. in Prozent der tatsächlich gemessenen Zeit). Nebel ist hier definiert als Zeitraum, in dem für einen Mittelungszeitraum von jeweils 30 Minuten das Maximum der Partikelkonzentration d N / d log r in einer Größenklasse mit einem Durchmesser > 0,6 $\mu \mathrm{m}$ liegt.

\begin{tabular}{|c|c|c|}
\hline Monat & gemessen $^{\text {a) }}$ & "Nebel" \\
\hline \hline Februar 2000 & $72 \%$ & $13,8 \%$ \\
\hline März & $76 \%$ & $20,6 \%$ \\
\hline April & $95 \%$ & $6,1 \%$ \\
\hline Mai & $96 \%$ & $4,5 \%$ \\
\hline Juni & $31 \%$ & $4,2 \%$ \\
\hline Juli & $64 \%$ & $6,8 \%$ \\
\hline August & $41 \%$ & $1,6 \%$ \\
\hline September & $95 \%$ & $4,4 \%$ \\
\hline Oktober & $75 \%$ & $11,2 \%$ \\
\hline November & $89 \%$ & $7,6 \%$ \\
\hline Dezember & $75 \%$ & $5,7 \%$ \\
\hline Januar 2001 & $38 \%$ & $4,9 \%$ \\
\hline Februar & $71 \%$ & $7,9 \%$ \\
\hline März & $92 \%$ & $11,0 \%$ \\
\hline April & $56 \%$ & $9,4 \%$ \\
\hline Juli & $39 \%$ & $2,5 \%$ \\
\hline August & $20 \%$ & $2,6 \%$ \\
\hline
\end{tabular}

a) nicht gemessen wurde bei technischen Problemen sowie bei Regenereignissen 


\subsubsection{Zeitlich hochaufgelöstes Signal der Partikelkonzentration}

Für die Anwendung der Eddy - Kovarianzmethode sind die mittleren Partikelkonzentrationen, die in den letzten beiden Abschnitten diskutiert wurden, nur von untergeordneter Bedeutung. Entscheidend für eine erfolgreiche Anwendung der Kovarianzmethode ist die Erfassung der Fluktuationen der Konzentration bei einer ausreichenden hohen zeitlichen Auflösung des Signals und mit einem ausreichend großen Signal-zu-Rauschen-Verhältnis. Wie in diesem Abschnitt gezeigt wird, können diese Forderungen durch das verwendete Partikelmessgerät (OPC) nur teilweise erfüllt werden.

Wie an Hand der Powerspektren klar erkannt werden kann, ist das Signal der Partikelkonzentration, wie es vom OPC aufgenommen wird, hochgradig verrauscht. Der Verlauf der spektralen Dichte folgt in der gewählten Darstellung (Produkt der Spektraldichte $P$ und der Frequenz $n$ als Funktion von $n$ ) für weite Frequenzbereiche der 1:1 Linie, was als Anzeichen für sogenanntes Weißes Rauschen ${ }^{15}$ gedeutet wird. Nur für Frequenzbereiche kleiner 0,02 Hz sind für einige Größenklassen Spektralverläufe zu erkennen, die tatsächlich als Resultat atmosphärischer Turbulenz verstanden werden können. Dies gilt insbesondere für die Größenklassen (in der Reihenfolge der Stärke der Abweichung von der 1:1-Linie) 0,30, 0,22 und 0,42 $\mu \mathrm{m}$. Bei diesen Klassen handelt es sich um die Bereiche mit den höchsten, mittleren Partikelkonzentrationen (Tabelle 7). Es kann deshalb festgehalten werden, dass die Stärke des Weißen Rauschens des Partikelsignals mit einer Abnahme der Konzentration zunimmt (Abb. 25, links).
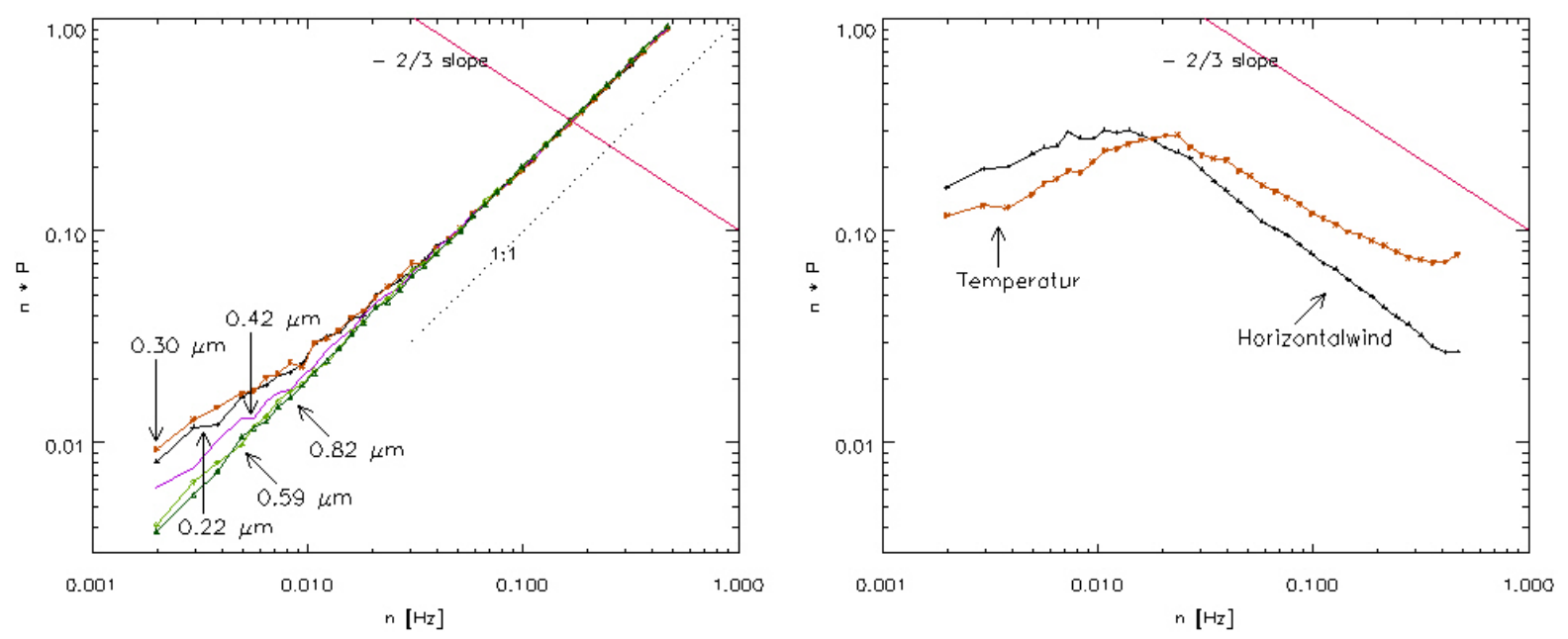

Abb. 25: Gemittelte Powerspektren für die Konzentration der fünf kleinsten Partikelklassen (links) sowie für horizontale Windgeschwindigkeit und Lufttemperatur (rechts). Zur Orientierung sind die -2/3 Linie und die 1:1 Linie miteingezeichnet. Für die Darstellung wurden 450 Zeitreihen mit $L<0,4$ verwendet ( $L=$ Monin-Obukhov-Mischungslänge).

\footnotetext{
${ }^{15}$ Weißes Rauschen: stochastischer Prozess, dessen statistische Eigenschaften von der Zeit unabhängig sind. Die Spektraldichte ist konstant, d.h. alle Frequenzen besitzen die gleiche Energie.
} 
Im Gegensatz zur Partikelkonzentration entsprechen die Powerspektren der Windgeschwindigkeit und der Luftemperatur sehr gut den theoretisch zu erwartenden Verläufen nach KAIMAL ET AL. (1972). Das Maximum der Spektraldichte befindet sich bei einer Frequenz von 0,01 bzw. 0,02 Hz. Für höhere Frequenzen folgen die Powerspektren der -2/3 Linie (Abb. 25, rechts). Für die Partikelspektren sind in Hinblick auf den turbulenten Transport der Partikel in der Atmosphäre natürlicherweise ähnliche Spektralverläufe zu erwarten. Da dies jedoch nicht der Fall ist, wird die Varianz der Partikelkonzentrationssignale offensichtlich ganz überwiegend von anderen Faktoren beeinflusst. Ursache und Auswirkungen dieses Phänomens werden im weiteren detailliert untersucht.

Bei der Einzelpartikelanalyse (vgl. Kapitel 1.1.1.4) wird das Partikelkonzentrationssignal aus der Summe der in einem ausgewählten, kleinen Luftvolumen gezählten Partikel sowie aus dem Volumenfluss durch das Gerät berechnet. Es handelt sich um eine abgeleitete Größe, die folglich mit statistischen Fehlern behaftet sein kann. Die Tatsache, dass das Partikelsignal insgesamt stark verrauscht ist, kann nun so interpretiert werden, dass diese statistischen Fehler der Einzelwerte für die vorliegende Untersuchung ausgesprochen groß sind. Dass diese Vermutung tatsächlich zutreffend ist, soll an Hand des folgenden Beispiels gezeigt werden.

Angenommen, die mittlere Partikelkonzentration $c_{\text {real }}$ an einem Ort in der Atmosphäre betrage konstant 180 Teilchen pro $\mathrm{cm}^{3}$. Dann ergibt sich aus dem pro Zeiteinheit untersuchten Luftvolumen $V_{\text {Scan }}$ und der betrachteten Zeitspanne $\Delta t$ die Anzahl der vom OPC zu zählenden Teilchen $P$ nach:

$$
P=c_{\text {real }} \cdot V_{\text {Scan }} \cdot \Delta t
$$

Bei einer Messfrequenz von $1 \mathrm{~Hz}$, wie sie für die Eddy - Kovarianzmethode notwendig ist, und einem $V_{\text {Scan }}$ von $0,05 \mathrm{~cm}^{3} / \mathrm{s}$ (abhängig vom tatsächlichen Volumenfluss durch den OPC) müssen in diesem Beispiel folglich genau 9 Teilchen in der Sekunde gezählt werden. Da die Partikel zufällig im Raum verteilt sind (Abb. 26), ist jedoch zu erwarten, dass die Zahl der tatsächlich gezählten Partikel von Messung zu Messung um den Erwartungswert von 9 schwankt. Dabei können immer nur "ganze" Partikel gezählt werden, das Ergebnis einer einzelnen Messung kann also nicht 8,8 oder 9,25 Teilchen lauten. Deshalb muss angenommen werden, dass die Verteilung der Messwerte über einen hinreichend langen Zeitraum betrachtet einer Poissonverteilung ${ }^{16}$ entspricht. Die Varianz dieser Messung $\sigma_{P}^{2}$ ist dann ebenfalls $c_{\text {real }} \cdot V_{\text {Scan }} \cdot \Delta t$ und beträgt also wiederum 9, die Standardabweichung folglich 3 Teilchen bzw. $30 \%$ für jeden einzelnen Messwert.

Betrachten wir nun das aus $P$ abgeleitete Partikelsignal $c$. Der Mittelwert der gemessenen Partikelkonzentration $\bar{c}$ ist $P /(V S c a n \cdot \Delta t)=180$ Teilchen $/ \mathrm{cm}^{3}$, also mit $c_{\text {real }}$ identisch. Diese wenig überraschende Feststellung besagt, dass eine über einem hinreichend langen Zeitraum

\footnotetext{
${ }^{16}$ Poisson-Verteilung (nach S.-D. Poisson): Theoretisches Verteilungsmodell für diskrete Zufallsvariablen, die nur ganzzahlige positive Werte und den Wert Null annehmen können.
} 
gemittelte Messung mit dem OPC die tatsächlichen Verhältnisse in der Atmosphäre tatsächlich korrekt wiedergibt. Die Varianz der gemessenen Partikelkonzentration $\sigma_{c}^{2}$ ergibt sich nach:

$$
\sigma_{c}^{2}=\frac{\sigma_{P}^{2}}{\left(V_{\text {Scan }} \Delta t\right)^{2}}=\frac{c_{\text {real }}}{V_{\text {Scan }} \Delta t}
$$

Für die angenommene Partikelkonzentration von 180 Teilchen $/ \mathrm{cm}^{3}$ und dem gegebenen Volumenfluss ist also $\sigma_{c}^{2}=3600$. Dies bedeutet aber, dass die Partikelkonzentration bei der sekündlichen Einzelmessung mit einer statistischen Sicherheit von nur $\pm 30 \%$ für jeden Messwert erfasst wird.

Abb. 26: Zufällige Verteilung der Partikel im Raum und beliebig gewählter Ausschnitt zur Bestimmung der Partikeldichte

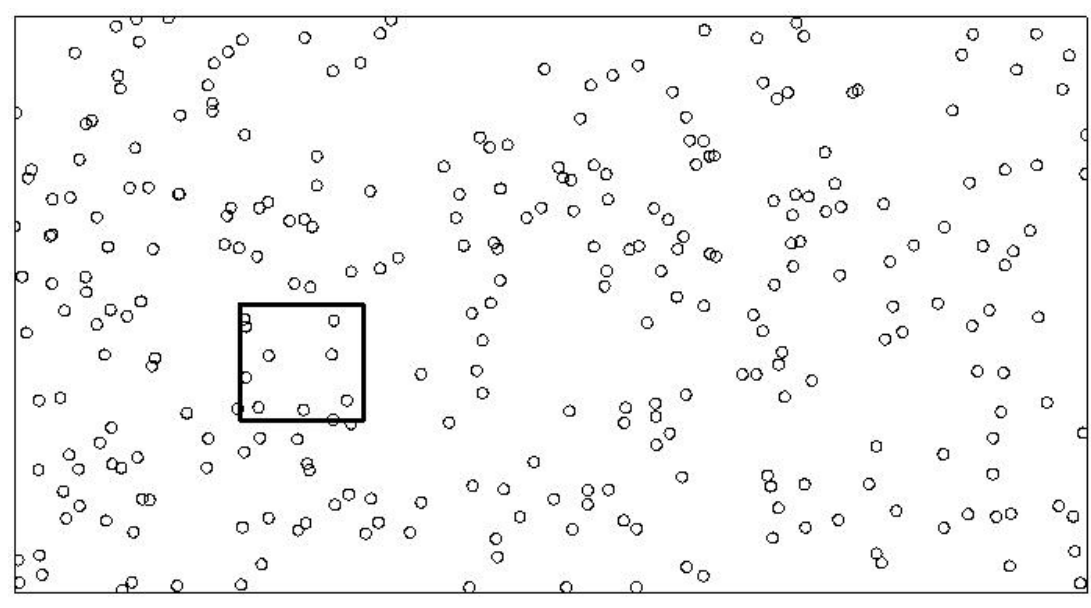

Diese Varianz - oder statistische Unsicherheit - des gemessenen Partikelsignals ist nicht Ausdruck einer Veränderung der realen Konzentration in der Atmosphäre (die ja als konstant angenommen wurde), sondern vielmehr das Ergebnis der Variabilität der räumlichen Verteilung der vorhandenen Teilchen. Die aus der räumlichen Variabilität folgende Messungenauigkeit ist um so größer, je kleiner die zu untersuchenden Partikelkonzentrationen sind. Dies wird in Tabelle 9 deutlich, in der die mittleren Konzentrationen und die Standardabweichung für die 12 Klassen des OPC zusammengestellt sind. Mit $25 \%$ ist die Standardabweichung für die Größenklasse mit der höchsten Partikelkonzentration am niedrigsten. Für die Klassen mit den kleinsten Konzentrationen wächst sie dagegen auf bis fast $3500 \%$ an.

Es muss betont werden, dass es sich bei dieser statischen Unsicherheit schneller Einzelmessungen im Prinzip um eine allgemeine Eigenschaft von Einzelpartikelanalysatoren handelt, nicht aber um ein spezielles Problem des verwendeten Messgeräts PCS-2000. Gerätespezifisch ist alleine die Größe des pro Zeitintervall gescannten Luftvolumens. Kann hier eine Vergrößerung erreicht werden, beispielsweise durch eine Erhöhung der Ansauggeschwindigkeit oder einer Vergrößerung des Messvolumens, wird die statistische Unsicherheit entsprechend Gleichung (35) reduziert. Dabei muss jedoch die Problematik von 
Koinzidenzfehlern ${ }^{17}$ berücksichtigt werden. Auch kann die zeitliche Dauer der erzeugten Streulichtimpulse in einem Einzelpartikelzähler nicht beliebig verkürzt werden, da die Impulse sonst nicht mehr von den Photosensoren registriert werden können. Dies schränkt die Maximalgeschwindigkeit, mit der die Probenluft durch den Analysator gesaugt werden kann, ebenfalls ein. Eine deutliche Verringerung der statistischen Unsicherheit kann lediglich bei der Verwendung von Partikelzählern erreicht werden, die die Partikelkonzentration nicht am Einzelpartikel, sondern am Partikelkollektiv bestimmen, da hier prinzipiell größere Luftvolumina pro Zeiteinheit untersucht werden.

Tabelle 9: Mittlere Konzentrationen und aus dem Messprinzip des OPC abgeleiteter Standardfehler der Konzentrationsmessung für die 12 Klassen des OPC

\begin{tabular}{|c|c|c|c|}
\hline Klasse & $\begin{array}{c}\text { mittlerer } \\
\text { Durchmesser } \\
(\mu \mathrm{m})\end{array}$ & $\begin{array}{c}\text { mittlere } \\
\text { Konzentration } \\
\left(1 / \mathrm{cm}^{3}\right)\end{array}$ & $\begin{array}{c}\text { mittlerer } \\
\text { Standardfehler } \\
(\%)\end{array}$ \\
\hline \hline 1 & 0,22 & 251,6 & 28,19 \\
\hline 2 & 0,30 & 320,6 & 24,98 \\
\hline 3 & 0,42 & 114,1 & 41,87 \\
\hline 4 & 0,59 & 31,79 & 79,32 \\
\hline 5 & 0,82 & 8,716 & 151,5 \\
\hline 6 & 1,14 & 4,962 & 200,8 \\
\hline 7 & 1,60 & 3,878 & 227,1 \\
\hline 8 & 2,23 & 1,809 & 332,5 \\
\hline 9 & 3,11 & 0,783 & 505,4 \\
\hline 10 & 4,34 & 0,242 & 908,6 \\
\hline 11 & 6,06 & 0,0381 & 2290 \\
\hline 12 & 8,46 & 0,0164 & 3484 \\
\hline
\end{tabular}

${ }^{\text {a) }}$ gesamter Messzeitraum ${ }^{\text {b) }}$ der Einzelmessung bei einer Messfrequenz von $1 \mathrm{~Hz}$ und dem gegebenen Messvolumen des OPC

\subsubsection{Bestimmung der Partikelflüsse}

Aus den im vorherigen Abschnitt dargestellten Eigenschaften der zeitlich hochaufgelösten Bestimmung der Partikelkonzentration mit einem Einzelpartikelanalysator ergibt sich die Frage, ob - für die gegebenen Konzentrationen - die geringe statistische Sicherheit, mit der die turbulenzbedingten Fluktuationen der Partikelkonzentration vom OPC erfasst werden, für eine zuverlässige Berechnung der Partikelflüsse mit Hilfe der Kovarianzmethode ausreicht.

\footnotetext{
${ }^{17}$ Koinzidenz (lat.): Zusammenfall, Zusammentreffen zweier Ereignisse (hier: gleichzeitiges Eintreffen zweier Partikel im Messvolumen)
} 
Diese Frage soll zunächst empirisch beantwortet werden. In Abb. 27 sind Tagesgänge der Depositionsgeschwindigkeit luftgetragener Partikel für die 6 kleinsten Partikelklassen des OPC für den 23. und 24. August 2000 aufgetragen, die nach der Berechnungsvorschrift der Eddy - Kovarianzmethode (Gleichung 23) ermittelt wurden. Die Partikelkonzentrationen der einzelnen Größenklassen waren an diesen beiden Tagen im allgemeinen etwas niedriger als in Tabelle 9; in ihrer Größenordnung entsprachen die Konzentrationen aber den dort angegebenen Werten. Für die Darstellung der Depositionsgeschwindigkeiten wurde bewusst kein einheitlicher Maßstab verwendet, da sich die berechneten Werte für die einzelnen Größenklassen um fast eine Größenordnung unterscheiden.
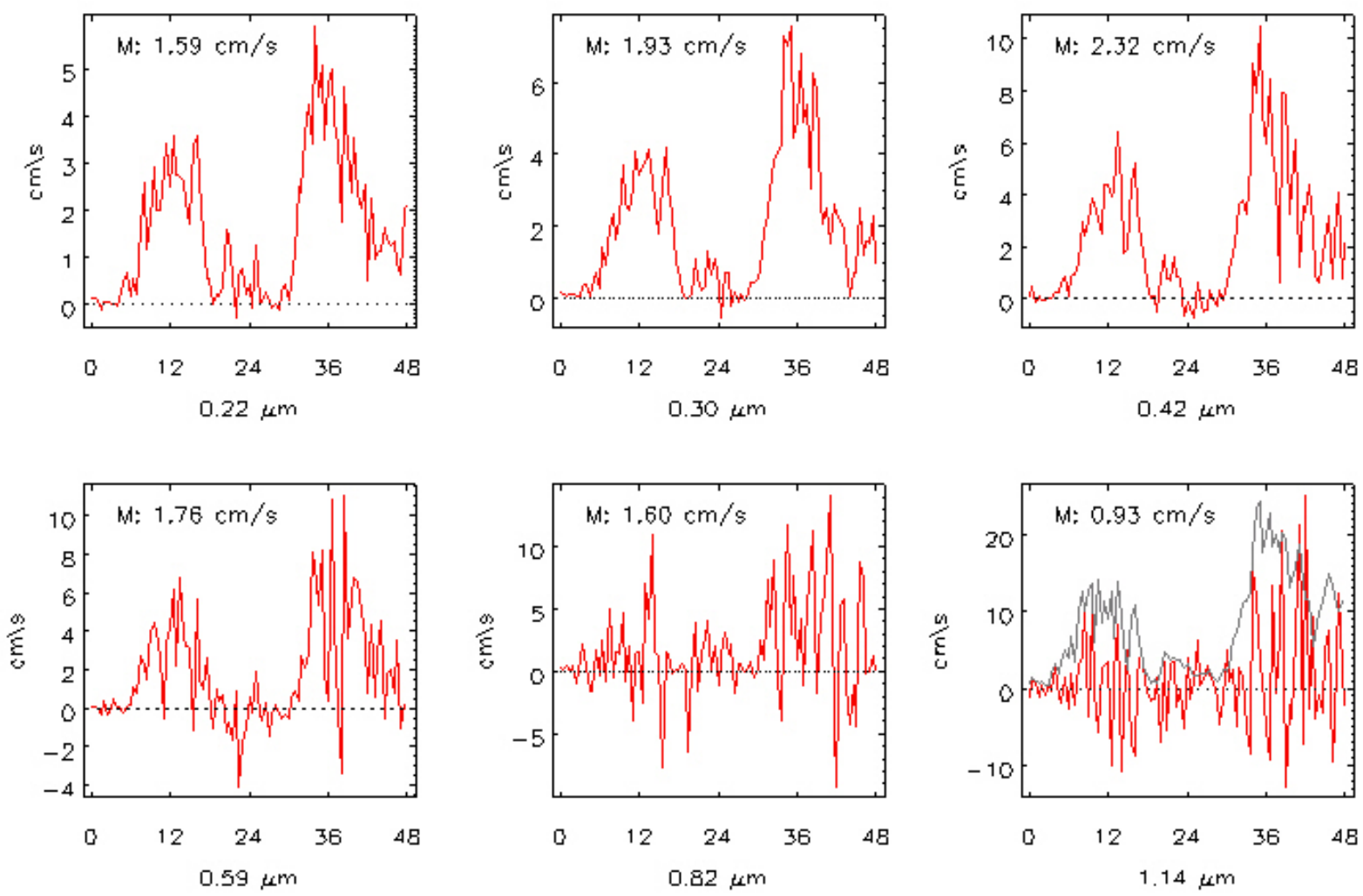

Abb. 27: Berechnete Tagesgänge der Depositionsgeschwindigkeit luftgetragener Partikel für die sechs kleinsten Größenklassen des OPC für den 23./24.8.00. Die graue Linie in der Graphik rechts unten beschreibt die theoretisch maximal mögliche Depositionsgeschwindigkeit (bei ausschließlich turbulentem Transport durch die Atmosphäre), berechnet als $r_{a}^{-1}=u_{*}^{2} / u$. M: arithmetisches Mittel.

Die ersten drei Klassen $(0,22 \mu \mathrm{m}, 0,30 \mu \mathrm{m}$ und $0,42 \mu \mathrm{m})$ zeigen insgesamt einen sehr deutlichen, in erster Näherung durchaus realistisch erscheinenden Tagesgang der Depositionsgeschwindigkeit. Dieser könnte beispielsweise mit dem Tagesgang der Turbulenz eine hineichende und überzeugende Erklärung finden. Die Beträge der Geschwindigkeit (arithmetische Mittel: 1,59, 1,93 und 2,32 cm/s) nehmen mit der Partikelgröße kontinuierlich zu, was ebenfalls als ein plausibles Ergebnis gedeutet werden darf. Die vierte Größenklasse $(0,59 \mu \mathrm{m})$ weist noch einen andeutungsweise akzeptablen Tagesgang auf, jedoch ist der Betrag der 
Depositionsgeschwindigkeit im arithmetischen Mittel mit 1,76 cm/s nun unerwarteter Weise niedriger als in Klasse drei. Zeitweise treten für die Klasse auch negative Werte auf, die wahrscheinlich als unrealistisch einzustufen sind. Für die Größenklasse fünf und insbesondere für Klasse sechs $(0,82$ und $1,14 \mu \mathrm{m})$ werden abwechselnd sowohl positive als auch negative Depositionsgeschwindigkeiten mit z.T. unrealistisch hohen Beträgen berechnet. Diese übersteigen für Klasse sechs vereinzelt die maximal möglichen Depositionsgeschwindigkeiten $\left(r_{a}^{-1}\right)$. Das arithmetische Mittel geht gegenüber der vorherigen Klasse vier weiter zurück. Insgesamt erwecken die Werte in beiden Klassen fast schon den Eindruck einer Zufallsreihe. Dies gilt - für die vorliegenden Partikelkonzentrationen - in noch verstärkter Weise für alle folgenden, größeren Kanäle des OPC (ohne Abbildung).

Die am Beispiel des 23./ 24. August exemplarisch gezeigten Merkmale der berechneten Werte der Depositionsgeschwindigkeit spiegeln sich auch in den gemittelten Tagesgängen der Depositionsgeschwindigkeit wieder. Sinnvolle mittlere Tagesgänge - mit ausschließlich positiven Beträgen, einem Maximum der Depositionsgeschwindigkeit in der Tagesmitte und einem Minimum in der Nacht - können nur für die ersten zwei, mit kleinen Abstrichen auch für die dritte und vierte Klasse aufgezeigt werden. Hier allerdings finden sich bereits vereinzelt negative Depositionsgeschwindigkeiten, die als langfristiges Mittel und insbesondere für die betrachtete Größenklasse unrealistisch sind. Für die vier größten gezeigten Partikelklassen verschwindet der Tagesgang immer mehr - ein Trend, der sich in den übrigen Partikelklassen weiter fortsetzt (Abb. 28).
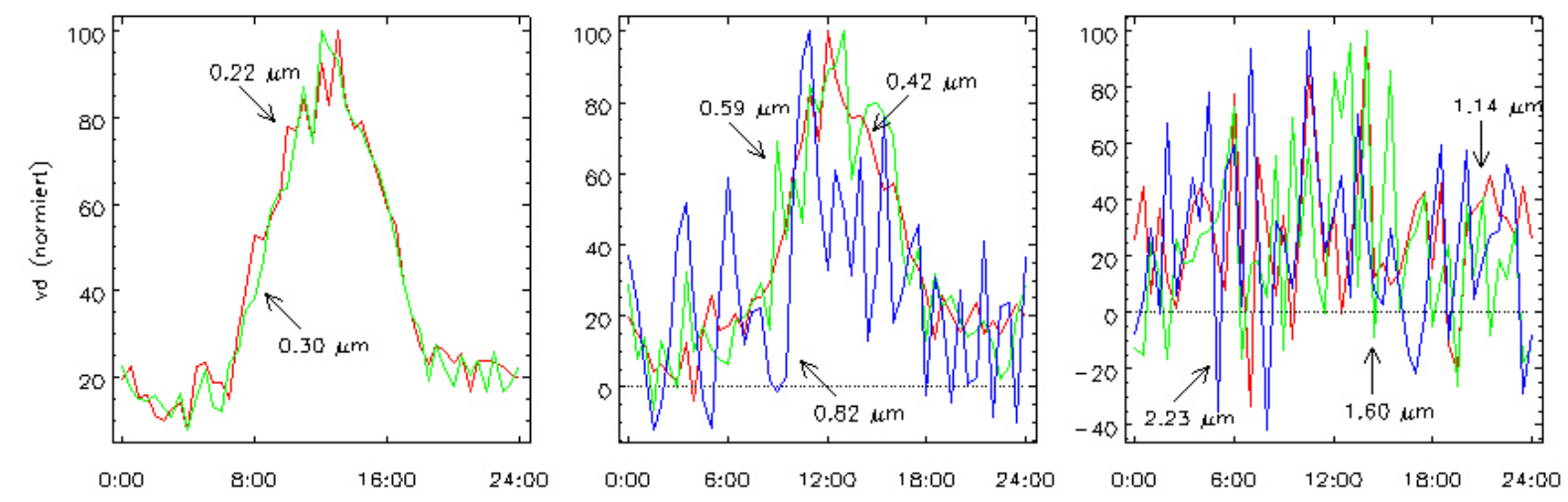

Abb. 28: Mittlere Tagesgänge der Depositionsgeschwindigkeit für die 8 kleinsten Klassen des OPC. Zum besseren Vergleich wurden die Tagesgänge jeweils mit dem Maximalwert normiert. Zeiträume mit einer relativen Feuchte $>95 \%$ sind bereits ausgeschlossen (siehe Abschnitt 4.1.5)

Es muss an dieser Stelle bereits geschlussfolgert werden, dass die Eddy - Kovarianzmethode unter den gegebenen Bedingungen durchaus nicht immer erfolgreich für die Bestimmung der Partikeldeposition eingesetzt werden kann. Vielmehr ist für ihren Einsatz wahrscheinlich die Einhaltung gewisser Rahmenbedingungen notwendig, die in den größeren Größenklassen offensichtlich nicht mehr oder nicht immer gegeben sind. In erster Näherung kann dabei vermutet werden, dass die auftretenden Flüsse nur dann realistisch mit dem OPC und unter 
Verwendung der Eddy-Kovarianzmethode gemessen werden können, wenn für die betrachteten Größenbereiche Partikelkonzentrationen von mindestens 30 Partikeln $/ \mathrm{cm}^{3}$ gegeben sind. Dieser Wert entspricht der mittleren Konzentration der vierten Größenklasse. Bei geringeren Konzentrationen, wie sie in allen größeren Klassen zu finden sind, ist das Signal-zu-Rauschen-Verhältnis des Konzentrationssignals bei einer Messfrequenz von $1 \mathrm{~Hz}$ offensichtlich nicht mehr groß genug für eine erfolgreiche Anwendung der Eddy Kovarianzmethode. Diese Vermutung soll im folgenden weiter untersucht und präzisiert werden. Ziel einer solchen Präzisierung ist es, ein Kriterium für die Korrektheit der nach der Berechnungsvorschrift der Eddy - Kovarianzmethode ermittelten Werte zu erarbeiten.

Es sei zunächst wieder der Spezialfall angenommen, dass die Partikelkonzentration in der Luft konstant sei, also insbesondere keine Schwankungen in Folge turbulenter Transporte aufweise. Die Varianz des mit dem OPC aufgenommenem Partikelsignals $c$ sei mit Gleichung (35) beschrieben. Die auftretenden Fluktuationen in diesem Signal sind dann also rein zufälliger Art, die sich aus dem Messprinzip des OPCs ergeben und nicht mit dem Signal der vertikalen Windgeschwindigkeit $w$ korrelieren. Betrachten wir nun das Produkt $w^{\prime} c^{\prime}$, so muss dieses über einen hinreichend langen Zeitraum $T$ gemittelt Null sein. Die Varianz des Produktes ergibt sich - rein als Folge des verwendeten Messprinzips - aus:

$$
\sigma_{w^{\prime} c^{\prime}}^{2}=\sigma_{c}^{2} \cdot \sigma_{w}^{2}=\frac{c_{\text {real }}}{V_{\text {Scan }} \Delta t} \cdot \sigma_{w}^{2}
$$

Dann kann der Standardfehler der Depositionsgeschwindigkeit $E_{v d}$ berechnet werden nach:

$$
E_{v_{d}}=\sqrt{\frac{\sigma_{w^{\prime} c^{\prime}}^{2}}{T}} / \bar{c}
$$

In Abb. 29 sind nach Gleichung 37 berechnete Standardfehler für verschiedene Standardabweichungen von $w$ dargestellt. Angenommen ist eine Messfrequenz von $1 \mathrm{~Hz}$ und ein Messvolumen von $1 / 20 \mathrm{~cm}^{3}$ pro Sekunde. Die vorausgesagten Fehler schwanken für den betrachteten Konzentrationsbereich und die betrachteten Standardabweichungen des Vertikalwindes zwischen 0,1 und $10 \mathrm{~cm} / \mathrm{s}$. Die niedrigsten Fehler finden sich bei hohen Partikelkonzentrationen und einer geringen Standardabweichung von $w$. Bei einer Standardabweichung von $1 \mathrm{~m} / \mathrm{s}$ (wie diese im Tagesverlauf bei Zeitreihen von 30 Minuten Länge häufig auftritt), kann die Depositionsgeschwindigkeit dagegen für Partikelkonzentrationen $<500$ Teilchen pro $\mathrm{cm}^{3}$ (bezogen auf eine Größenklasse) niemals mit einer höheren Genauigkeit als mit $\pm 0,5 \mathrm{~cm} / \mathrm{s}$ bestimmt werden.

Es ist nun die Frage, ob sich die Berechnungsvorschriften (36) und (37) auch auf den allgemeineren Fall des Vorhandenseins eines vertikalen Partikelflusses ungleich Null übertragen lassen. Wenn dies der Fall wäre, könnte mit dem gefundenen Ansatz die statistische Unsicherheit als Funktion der Partikelkonzentration, der Standardabweichung der vertikalen 
Windgeschwindigkeit, der Größe des pro Zeitintervall gescannten Volumens und der Messfrequenz vorausgesagt werden, die sich bei der Bestimmung der Depositionsgeschwindigkeit mit einem Einzelpartikelzähler allein aus dem Prinzip der Einzelpartikelanalyse ergibt.

Abb. 29: Vorausgesagte Standardfehler für die Depositionsgeschwindigkeit als Funktion der Partikelkonzentration für verschiedene Standardabweichungen der vertikalen Windgeschwindigkeit (von unten nach oben: $0,2,0,4,0,6$, 0,8 und $1 \mathrm{~m} / \mathrm{s}$ ) bei einer Messfrequenz von $1 \mathrm{~Hz}$ und einem Messvolumen von $1 / 20 \mathrm{~cm}^{3}$ pro Sekunde

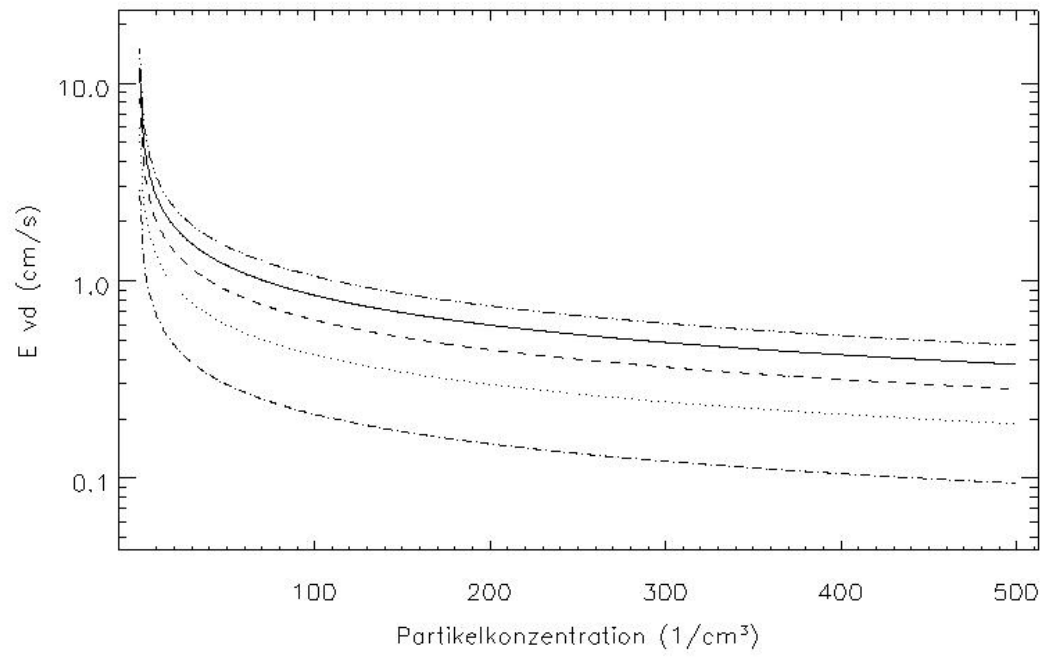

Diese Frage soll empirisch überprüft werden, indem für die einzelnen Halbstundenwerte der vorausgesagte Standardfehler mit dem realen Standardfehler der berechneten Depositionsgeschwindigkeit verglichen wird. Die Berechnung des Fehlers erfolgt wiederum nach Gleichung (37), jedoch wird für $\sigma_{w^{\prime} c^{\prime}}^{2}$ der real beobachtete Betrag verwendet (Abb. 30).
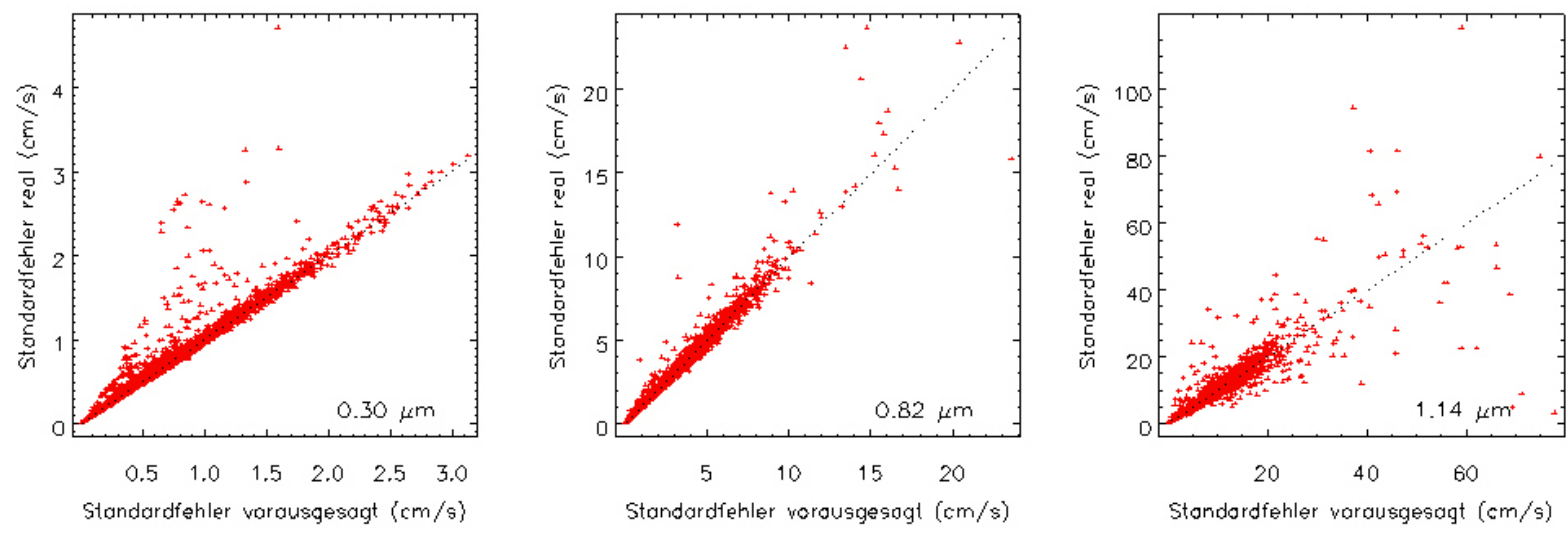

Abb. 30: Vergleich des vorausgesagten mit dem real beobachteten Standardfehler der Depositionsgeschwindigkeit für drei ausgewählte Größenklassen

Es zeigt sich, dass der real beobachtete Standardfehler der Depositionsgeschwindigkeit in aller Regel sehr gut mit dem vorausgesagten Fehler übereinstimmt. Die Unsicherheit der berechneten Depositionsgeschwindigkeiten ist mit Werten von bis zu 4 (resp. 20 und 80) cm/s 
also wirklich sehr hoch. Nur in einigen Fällen weicht der reale Fehler von dem vorausgesagten deutlich ab und ist dann, von einer geringen Anzahl von Halbstundenwerten abgesehen, sogar noch größer als der vorausgesagte Betrag. In diesen Fällen sind wahrscheinlich weitere Unzulänglichkeiten des Messvorgangs für den Unterschied verantwortlich. Am schlechtesten ist die Übereinstimmung für die größte der gezeigten Partikelklassen $(1,14 \mu \mathrm{m})$. Die Fehler in dieser Klasse sind mit Werten von häufig deutlich mehr als $10 \mathrm{~cm} / \mathrm{s}$ jedoch in jedem Fall bereits so hoch, dass sie die angenommene mittlere Depositionsgeschwindigkeit von $1 \mathrm{~cm} / \mathrm{s}$ bei weitem übersteigen.

Es kann geschlussfolgert werden, dass die statistische Unsicherheit bei der schnellen Konzentrationsmessung mit dem OPC eine signifikante Unsicherheit bei der Bestimmung der Depositionsgeschwindigkeit mit Hilfe der Kovarianzmethode direkt nach sich zieht. Diese Unsicherheit ist für die einzelnen Größenklassen und für unterschiedliche Randbedingungen unterschiedlich stark ausgeprägt. Generell gilt: je niedriger die Partikelkonzentrationen in den Klassen sind, um so unsicher sind die berechneten Depositionsgeschwindigkeiten.
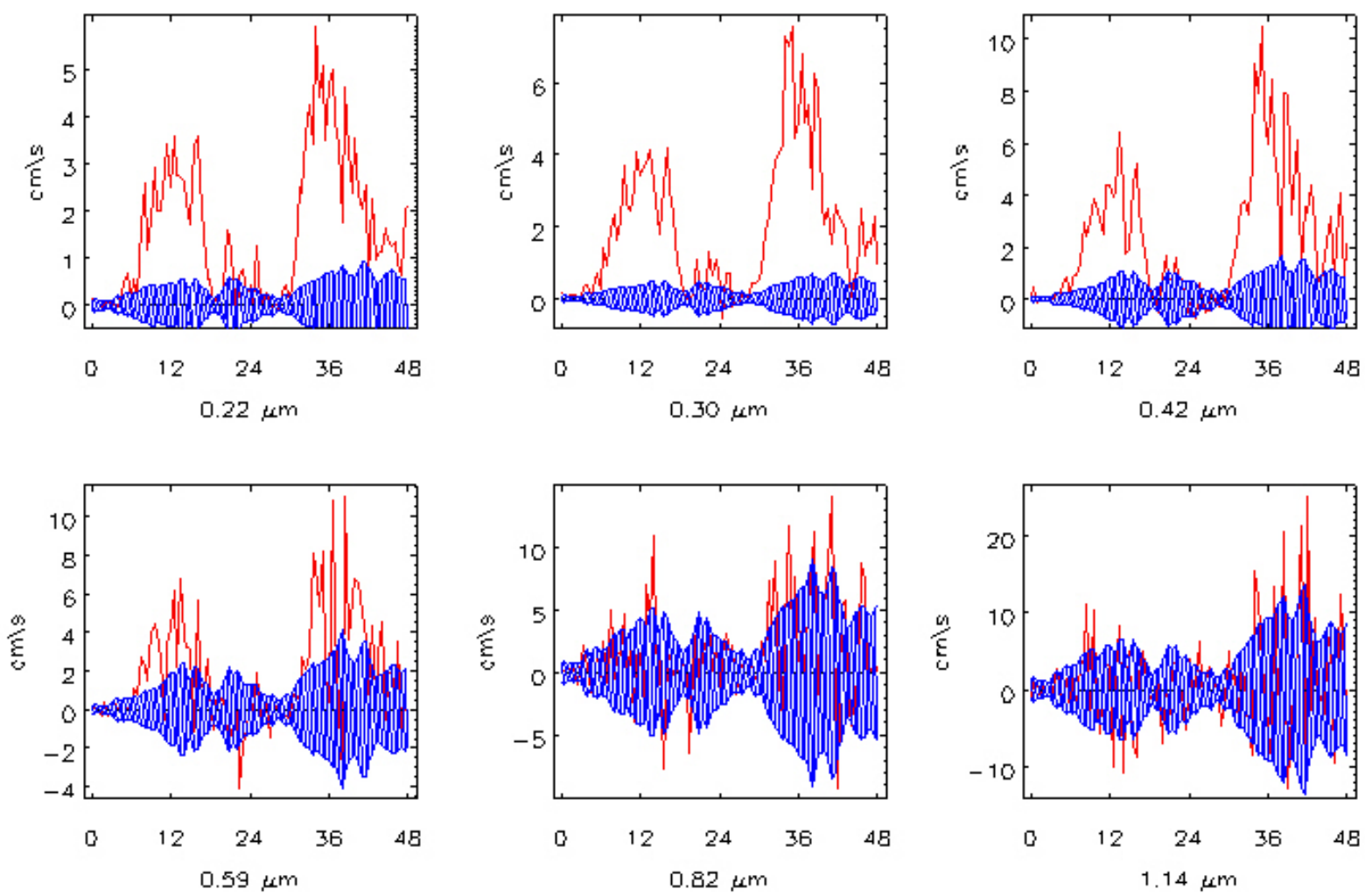

Abb. 31: Berechnete Tagesgänge der Depositionsgeschwindigkeit luftgetragener Partikel für die sechs kleinsten Größenklassen des OPC für den 23./24.8.00 (rot) sowie nach Gleichung (37) vorausgesagter Standardfehler der Messwerte (blau, beidseitig zur x-Achse aufgetragen)

Es gilt nun, den Betrag dieser Unsicherheit zu bewerten. Da die tatsächlichen Depositionsgeschwindigkeiten nicht bekannt sind, kann dies nur in Bezug zu den gemessenen Werten 
geschehen. Die eingangs betrachteten Depositionsgeschwindigkeiten vom 23. und 24. August werden deshalb in Abb. 31 mit den vorausgesagten Standardfehler verglichen. Es zeigt sich, dass sich für die Kanäle 5 und 6 fast alle berechneten Depositionsgeschwindigkeiten in dem durch den Standardfehler überdeckten Bereich befinden. Dies bedeutet jedoch, dass aus diesen Werten noch nicht einmal die Richtung des tatsächlichen Partikelflusses sicher bestimmt werden kann. Für Kanal 4 gilt diese Aussage für einen Teil der berechneten Werte, für Kanal 1 bis 3 immerhin noch für die meisten Nachtwerte. Wiederum kann an Hand der Resultate vom 23. und 24. August exemplarisch abgeleitet werden, dass die Anwendung der Eddy-Kovarianzmethode unter den gegebenen Umständen und mit dem gegebenen Messgerät nur für einige (hier: für die ersten drei) Klassen des OPC sinnvoll möglich ist.
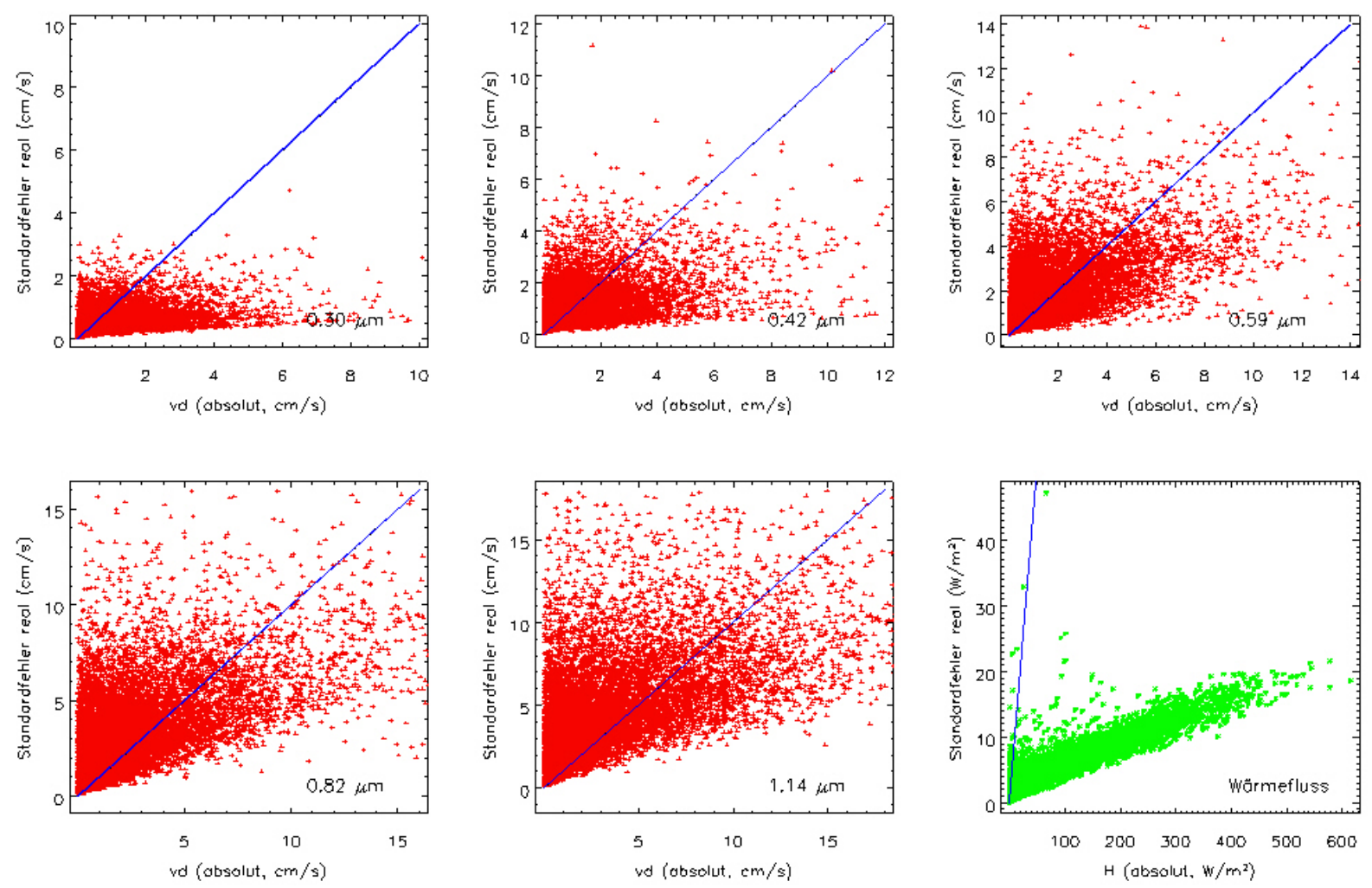

Abb. 32: Vergleich der gemessenen Depositionsgeschwindigkeit (absolut) mit dem Standardfehler für fünf ausgewählte Größenklassen des OPC (nur für Luftfeuchten $<96 \%$ ). rechts unten: Vergleich des bestimmten Wärmeflusses (absolut) mit dem zugehörigen Standardfehler. Blau: 1:1 Linie

Dass das Verhältnis zwischen ermittelter Depositionsgeschwindigkeit und dem Standardfehler mit abnehmender Partikelkonzentration (also hier in der Regel mit zunehmender Partikelgröße) immer ungünstiger wird, ist auch in Abb. 32 zu erkennen. Hier ist der Standardfehler (real) gegen den Absolutbetrag der gemessenen Depositionsgeschwindigkeit aufgetragen. Berücksichtigt wurden alle Halbstundenwerte zwischen dem 1. Januar 2000 und dem 30. August 2001. Es zeigt sich, dass selbst in der Größenklasse mit der durchschnittlich höchsten 
Partikelkonzentration (mittlerer Durchmesser 0,30 $\mu \mathrm{m}$ ) zahlreiche (37\%) Halbstundenbeobachtungen existieren, in denen der Standardfehler größer ist als die berechnete Depositionsgeschwindigkeit selbst. In der Klasse vier mit einem mittleren Durchmesser von $0,59 \mu \mathrm{m}$ gilt dies bereits für $58 \%$ aller Werte, in der Klasse mit dem mittleren Durchmesser von $1,14 \mu \mathrm{m}$ sogar für $65 \%$ aller Werte. Eine äquivalente Betrachtung für den fühlbaren Wärmefluss bestätigt, dass es sich ausschließlich um ein auf die Bestimmung des Partikelflusses beschränktes Problem handelt, da das Verhältnis zwischen Messwert und Standardfehler im Falle des fühlbaren Wärmeflusses (unter gleichen Bedingungen bestimmt) sehr viel günstiger (größer) ausfällt als beim Partikelfluss.

Es ist nun vermutet worden, dass unter Verwendung der Berechnungsvorschrift für die relaxed eddy accumulation (REA, Abschnitt 2.3) eine höhere Zuverlässigkeit bei der Flussberechnung erreicht werden könne. Die Berechnungsvorschrift lautet (identisch mit Gleichung 33):

$$
F=\beta \sigma_{w}\left(\overline{C^{+}}-\overline{C^{-}}\right)
$$

$$
\text { mit: } \begin{aligned}
\sigma_{w} & \text { Standardabweichung der vertikalen Windgeschwindigkeit } \\
\frac{\beta}{\overline{C^{+}}} & \begin{array}{l}
\text { Proportionalitätskonstante (dimensionslos, ca. } 0,56 \text { bis } 0,6) \\
\text { mittlere Spurenstoffkonzentration bei aufsteigendem Vertikalwind } \\
\overline{C^{-}}
\end{array} \text {mittlere Spurenstoffkonzentration bei absteigendem Vertikalwind }
\end{aligned}
$$

Auch für diesen Ansatz soll im folgenden der Standardfehler für die Depositionsgeschwindigkeit vorausgesagt werden. Da $\beta$ konstant ist und $\sigma_{w}$ von einem unabhängigen Gerät bestimmt wird, reduziert sich die Problemstellung zu der Frage, mit welcher Genauigkeit die Konzentrationsdifferenz bestimmt werden kann. Hierfür sei angenommen, dass der Vertikalwind in jeweils gleich langen Zeitspannen auf- bzw. absteigt. Dann kann der Standardfehler für $\overline{C^{+}}$bzw. $\overline{C^{-}}$bestimmt werden nach:

$$
E_{\overline{C^{x}}}=\sqrt{\frac{\frac{c_{\text {real }}}{V_{\text {scan }} \cdot \Delta t}}{T / 2}}
$$

Daraus ergibt sich der Standardfehler der Depositionsgeschwindigkeit nach:

$$
E_{v_{d}}=\left(\sqrt{2} \cdot E_{\overline{C^{x}}} \cdot \beta \cdot \sigma_{w}\right) / \bar{c}
$$

Wie Abb. 33 zeigt, liegt dieser für die betrachteten Partikelkonzentrationen in ähnlichen Größenordnungen wie derjenige der normalen Flussberechnung. Er ist sogar noch etwas größer als letzterer, so dass mit einer Flussberechnung "alternativ" nach dem Prinzip der REA keine höhere Zuverlässigkeit der Resultate erreichbar ist.

Aus Abb. 29 (und Abb. 33) folgt, dass es - entgegen der ersten Hypothese - keine allgemeingültige, untere Grenze der Partikelkonzentration gibt, ab der eine Anwendung der 
Eddy - Kovarianzmethode auf Grund der statistischen Unsicherheit der Einzelpartikelanalyse und des damit verbundenen Standardfehlers bei der Bestimmung der Depositionsgeschwindigkeit grundsätzlich nicht mehr möglich wäre. Vielmehr muss stets die Kombination aus der Standardabweichung der vertikalen Windgeschwindigkeit, der mittleren Partikelkonzentration, des pro Zeiteinheit gescannten Volumens und der Messfrequenz betrachtet werden, um die unvermeidliche Unsicherheit bei der Flussberechnung bestimmen zu können. Für die Untersuchungen der vorliegenden Arbeit muss aber konstatiert werden, dass die vorgefundenen, relativ niedrigen Partikelkonzentrationen in der Umgebungsluft zusammen mit den Eigenschaften des zur Verfügung stehenden Messgerätes insgesamt eine sehr hohe Unsicherheit bei der Partikelflussbestimmung bewirkt haben. Dies gilt insbesondere für alle Partikelklassen $>0,59 \mu \mathrm{m}$ Durchmesser.

Abb. 33: Vorausgesagte Standardfehler für die Depositionsgeschwindigkeit wie Abb. 29, jedoch bei Auswertung der Daten nach der Berechnungsvorschrift der relaxed eddy accumulation

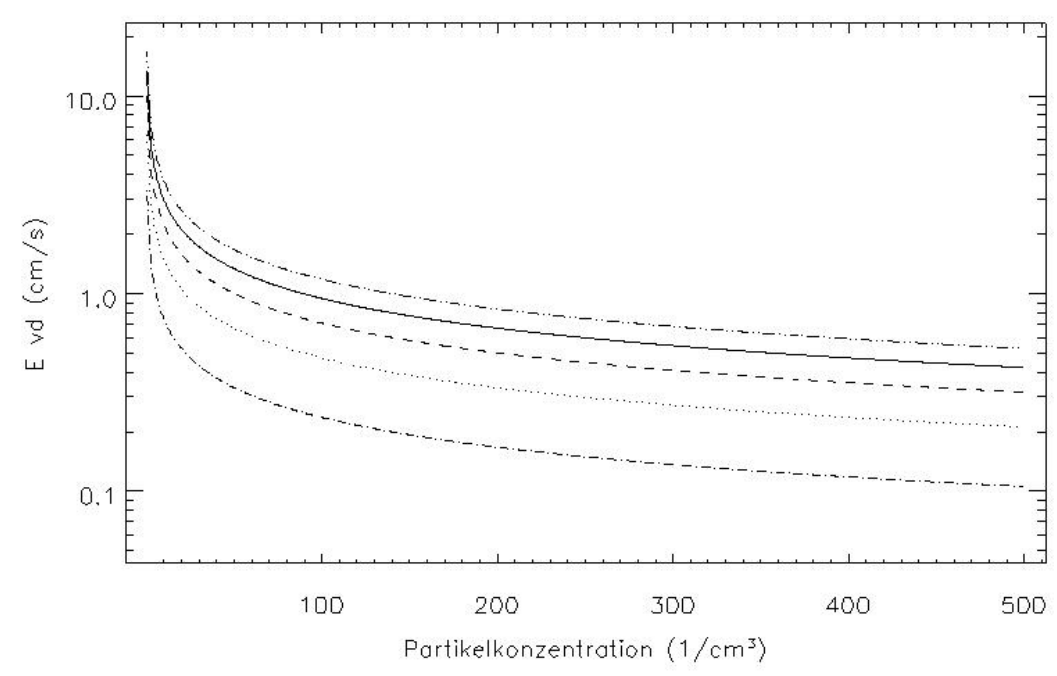

\subsubsection{Einfluss der relativen Luftfeuchte auf die Bestimmung der Partikelflüsse}

Für weite Bereiche der relativen Luftfeuchte $(<50 \%$ bis ca. $96 \%)$ ist kein statistischer Zusammenhang zwischen den berechneten Depositionsgeschwindigkeiten und der Luftfeuchte zu erkennen. Bei Feuchten $>96 \%$ kommt es jedoch zu einer Abweichung von dieser Regel: dann werden in allen Größenklassen Depositionsgeschwindigkeiten ermittelt, deren Beträge um ein Vielfaches größer sind als bei Luftfeuchten < $96 \%$ (Abb. 34 und Abb. 35).

In den kleineren Größenklassen $(0,22$ und $0,30 \mu \mathrm{m})$ werden dabei unter den genannten Bedingungen in erster Linie sehr große Depositionsgeschwindigkeiten ( $>$ Null) gemessen, die einen raschen Transport von Teilchen aus der Atmosphäre zum Erdboden hin suggerieren. Dagegen werden die größeren Partikel $(>0,59 \mu \mathrm{m})$ den Messergebnissen nach mit Geschwindigkeiten von bis zu $60 \mathrm{~cm} / \mathrm{s}$ aus dem Waldbestand in die Atmosphäre hinein befördert. Beide Vorgänge sind insbesondere in Anbetracht der großen Beträge der berechneten Transportgeschwindigkeit physikalisch nicht sinnvoll zu erklären. Es kann deshalb gefolgert werden, 
dass es sich um Messfehler handelt. Diese Phänomene, im folgenden als Scheinflüsse bezeichnet, sollen näher untersucht werden.

Abb. 34: Depositionsgeschwindigkeiten (geglättet) für den 25.11.00. An diesem Tag trat zwischen 4:00 und 12:00 Uhr bei hoher relativer Luftfeuchte ein Nebelereignis auf (vgl. Abb. 18), die Depositionsgeschwindigkeiten weichen für diesen Zeitraum deutlich von den übrigen Werten ab.

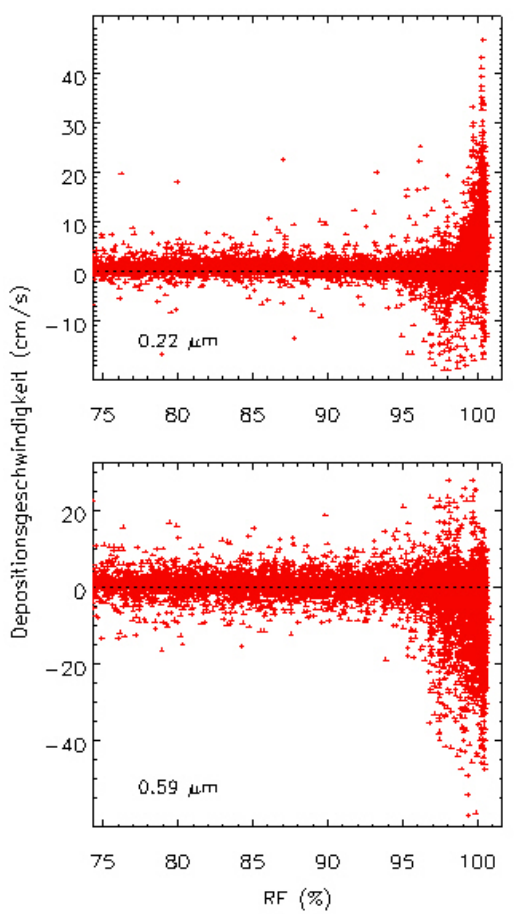

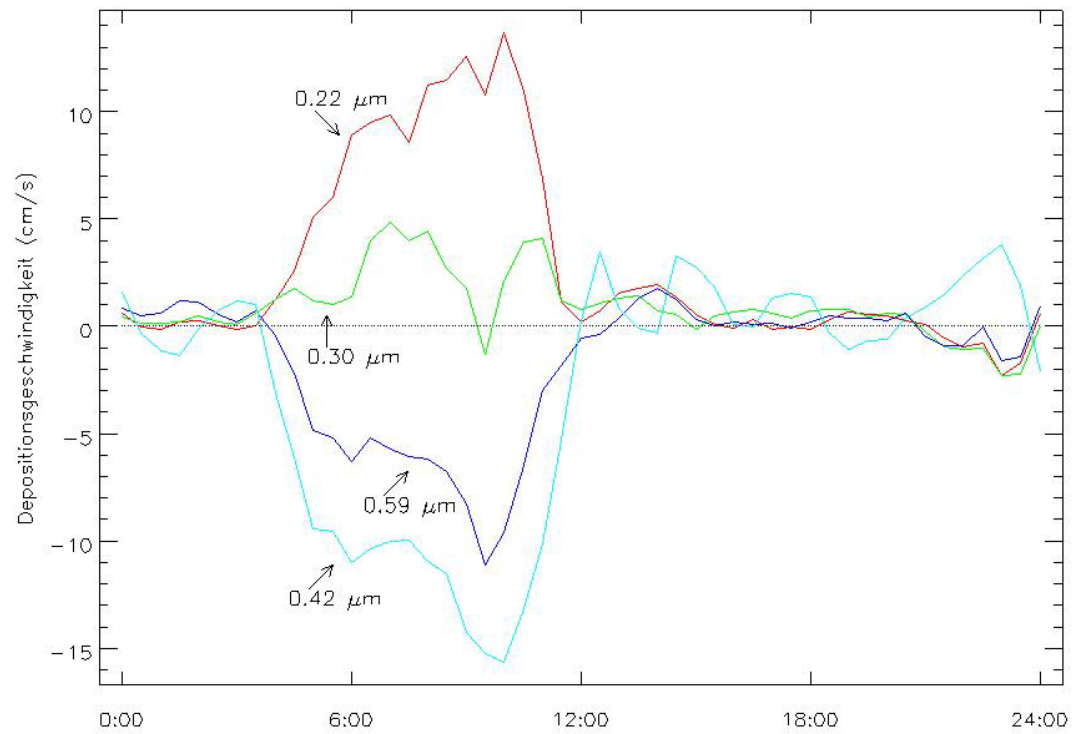
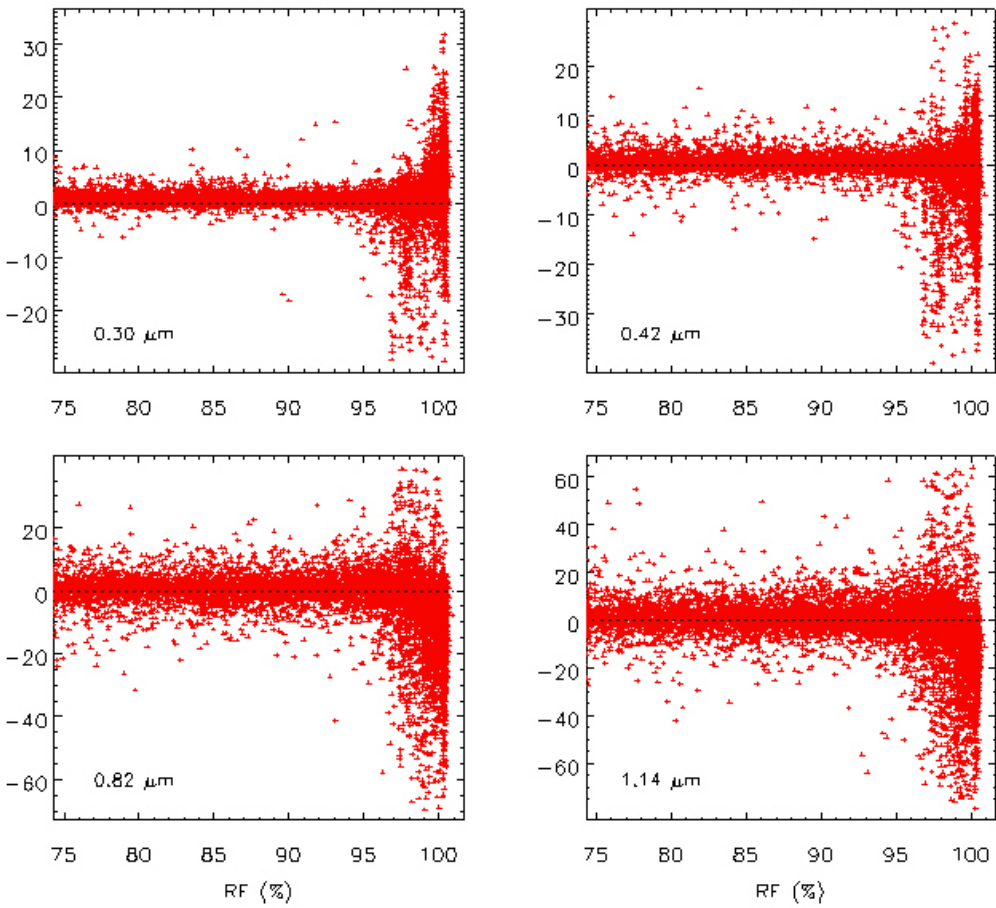

Abb. 35: Berechnete Depositionsgeschwindigkeiten für die sechs kleinsten Partikelklassen als Funktion der relativen Feuchte der Umgebungsluft (Halbstundenwerte vom 1.1.00 bis 1.9.01) 
Bereits in Abb. 22 und 22 ist zu erkennen, dass bei hohen relativen Luftfeuchten - parallel zu der Verschiebung des Konzentrationsmaximums im Partikelspektrum - ungewöhnlich große Fluktuationen der Konzentration in den einzelnen Partikelklassen auftreten. Es kann vermutet werden, dass diese Fluktuationen die offensichtlich unkorrekte Bestimmung des Partikelflusses verursachen. Voraussetzung hierfür ist, dass die Konzentrationsfluktuationen mit denen des vertikalen Windes korrelieren, obwohl sie ursächlich nicht mit einem turbulenten Transport von Partikeln zwischen Vegetation und Atmosphäre in Zusammenhang stehen.

Als Ursache der beobachteten Konzentrationsfluktuationen kann die Eigenschaft luftgetragener Partikel angenommen werden, ihre Größe in Abhängigkeit von der relativen Luftfeuchte zu verändern. Diese Eigenart ist besonders stark bei hohen relativen Luftfeuchten ausgeprägt, also bei einer (fast) vollständigen Sättigung der Luft mit Wasserdampf (FITZGERALD, 1975). Für das Auftreten von Scheinflüssen müssen die Partikel in der Lage sein, den Fluktuationen der relativen Feuchte auch ausreichend schnell zu folgen. Dies kann für Teilchen mit einem Durchmesser $<1 \mu \mathrm{m}$ als gegeben angenommen werden: Partikelgröße und relative Feuchte befinden sich hier quasi in einem ständigen Gleichgewicht (FAIRALL, 1984). Nun kann weiter angenommen werden, dass die Fluktuationen der relativen Feuchte mit denen der vertikalen Windgeschwindigkeit korreliert sind. Ist dies der Fall, und folgen die Konzentrationsänderungen in den Partikelklassen denen der relativen Feuchte, dann sind auch die Fluktuationen des Partikelsignals mit dem Vertikalwind korreliert, ohne dass notwendigerweise ein realer Partikeltransport zwischen Vegetation und Atmosphäre stattfindet.

Abb. 36: Partikelkonzentration für vier ausgewählte Größenklassen bei einer relativen Luftfeuchte $>98 \%$ (gepunktet) als Funktion der Zeit (x-Achse: Sekunden). Die Kurven wurden leicht geglättet. Zum Verlauf der relativen Feuchte siehe Fußnote auf S.68.

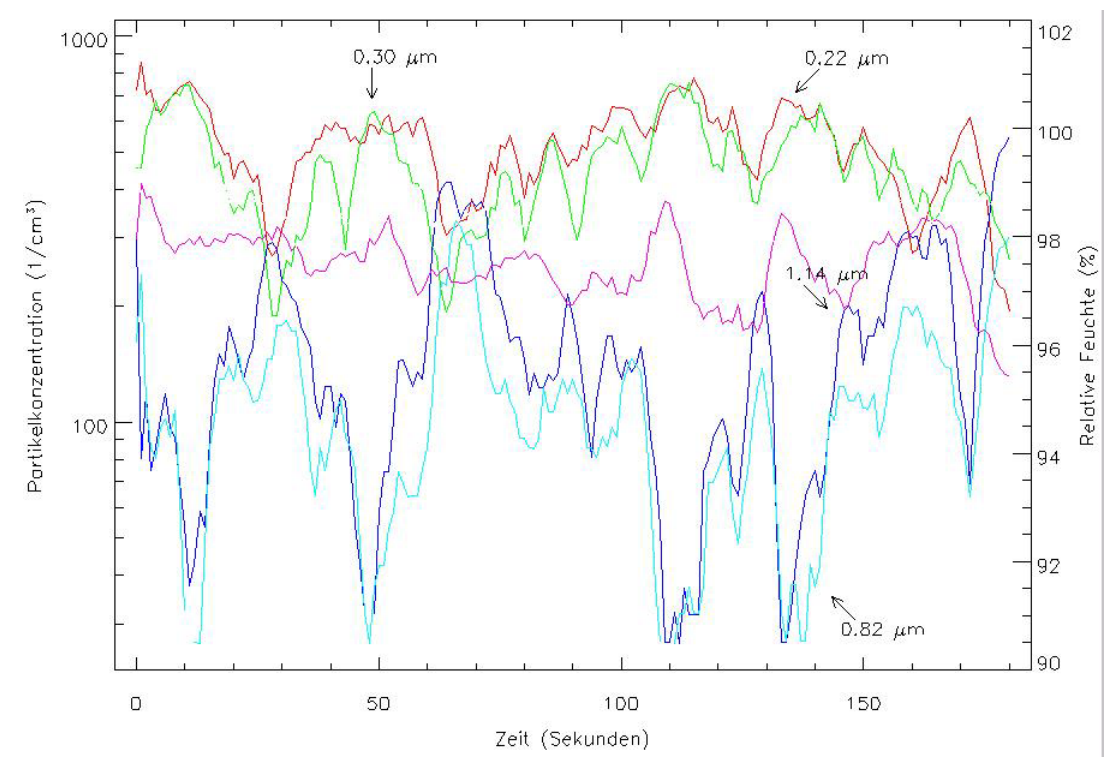

Wie Abb. 36 beispielhaft zeigt, sind die Fluktuationen der kleineren Partikel (0,22 und $0,30 \mu \mathrm{m}$ Partikeldurchmesser) im Falle hoher relativer Luftfeuchten mit denen der größeren Partikel (hier: 0,82 und 1,14 $\mu \mathrm{m}$ ) negativ korreliert. Dies deutet darauf hin, dass eine größere Anzahl von Teilchen wahrscheinlich auf Grund von Fluktuationen der relativen Luftfeuchte 
kontinuierlich seine Größe verändert und ständig zwischen den Größenklassen "hin- und herwandert". Bei Erreichen einer kritischen relativen Feuchte nahe 100 \% absorbieren die vorhandenen kleineren Partikel in kurzer Zeit große Mengen an Wasser aus der Umgebungsluft. Dementsprechend steigt die Konzentration der größeren Partikel sprunghaft an, während die der kleineren abfällt. Kommt es dann wieder zu einer Reduzierung der Luftfeuchte, geben die ursprünglich kleineren Teilchen das aufgenommen Wasser an die Umgebung ab, die feuchtebedingten Konzentrationsänderungen werden also wieder korrigiert. Dieses kontinuierliche Wandern fungiert aus Sicht einer einzelnen Größenklasse als Partikelquelle bzw. Partikelsenke. Damit aber ist eine wichtige Voraussetzung aller mikrometeorologischen Methoden zur Bestimmung vertikaler Flussdichten verletzt: die Abwesenheit zusätzlicher Quellen und Senken zwischen der Messebene und dem zu untersuchenden Bestand. Hiermit ist die formale Begründung für die Fehlabschätzung der Kovarianzmethode gegeben.

Die Abhängigkeit der Richtung der beobachteten Scheinflüsse von der Partikelgröße soll Rückschlüsse auf die Ursache der Fluktuationen der relativen Feuchte geben. Zunächst sei angenommen, dass die Änderungen der relativen Feuchte auf Fluktuationen der Wasserdampfdichte $q$ zurückzuführen sei. Es kann davon ausgegangen werden, dass der latente Wärmefluss $E$ (vgl. Gleichung 19) in Situationen mit einer großen relativen Luftfeuchte - also im Extremfall bei Nebel - klein und tendenziell eher negativ, also zur Erdoberfläche hin gerichtet ist. Dies bedeutet, dass die Wasserdampfdichte $q$ mit der vertikalen Windgeschwindigkeit $w$ negativ korreliert ist, also $\overline{w^{\prime} q^{\prime}}<0$. Gleichzeitig werden große Flüsse kleiner Teilchen zur Erdoberfläche hin bestimmt, es gilt also $\overline{w^{\prime} c^{\prime}}<0$ für kleinere Partikel. Demnach ist $c$ für kleinere Partikel positiv korreliert mit $q$. Würde nun die relative Luftfeuchte hauptsächlich auf Grund der Änderungen der Wasserdampfdichte fluktuieren, würde dies eine Zunahme der Partikelzahl kleinerer Partikel (und umgekehrt eine Abnahme größerer Partikel) mit der Zunahme der relativen Feuchte bedeuten. Dies entspricht jedoch nicht den Ergebnissen der bisherigen Überlegungen.

Es wird deshalb angenommen, dass die Fluktuationen der relativen Feuchte nicht auf Fluktuationen der Wasserdampfdichte sondern auf Änderungen der Lufttemperatur zurückzuführen sind. Wenn diese Änderungen von einem turbulenten Transport verursacht würden, müssten Zeiträume mit großen relativen Feuchten und daraus resultierenden Scheinflüssen immer mit einem negativen fühlbaren Wärmefluss verbunden sein. Dies ist sicher nicht gegeben. Eine plausiblere Erklärung könnte dagegen daraus abgeleitet werden, dass Luftpakete sich beim Aufsteigen abkühlen und umgekehrt beim Zurückströmen zur Erdoberfläche erwärmen. In einem aufsteigenden Luftpaket muss deshalb die relative Feuchte ansteigen, was bei Erreichen eines entsprechenden kritischen Punktes zu einer Erhöhung der Konzentration der größeren Partikel und zu einer Erniedrigung der kleineren Partikel führen kann. Beim Absteigen der Luft ist mit der Zunahme der Lufttemperatur und damit einer Abnahme der relativen Feuchte entsprechend die gegenteilige Situation zu erwarten. Damit wäre die Konzentration der größeren Partikel mit der vertikalen Windgeschwindigkeit positiv korreliert, was die Bestimmung eines scheinbaren Partikelflusses in die Atmosphäre hinein 
Resultate der eigenen Messungen

zur Folge hat. Die Konzentration der kleineren Partikel wäre dagegen negativ mit der relativen Feuchte korreliert, es werden also Scheinflüsse zur Erdoberfläche hin suggeriert.

Auch wenn die Entstehung der gemessenen Scheinflüsse in Situationen mit einer großen relativen Luftfeuchte mit Hilfe des obigen Ansatzes erklärt werden kann, gibt es keine Möglichkeit, die echten Partikelflüsse beispielsweise über einen Modellansatz von den Scheinflüssen zu trennen und damit Aussagen über den tatsächlichen Partikeltransport während der betrachteten Zeiträume zu treffen. Es bleibt deshalb nichts anderes übrig, als die berechneten Flüsse zu verwerfen. Als Kriterien hierzu können das Erreichen einer bestimmten kritischen relativen Feuchte oder das Auftreten einer negativen Korrelation zwischen größeren und kleineren Partikeln Verwendung finden. Für die vorliegende Arbeit wurde eine Kombination aus beiden Kriterien gewählt. Zusammen mit den Ausfällen, die durch Regen verursacht wurden, sind auf diese Weise knapp 40 \% der aufgezeichneten Daten ausselektiert worden.

\subsubsection{Verbesserung der Datenqualität: Modifikation des Datensatzes}

Für eine detaillierte Analyse der bestimmten Depositionsgeschwindigkeiten ist es zweckmäßig, korrektere von weniger korrekten Messwerten zu trennen. Nur auf diese Art und Weise kann die Basis für eine sinnvolle Prüfung etwaiger Zusammenhänge und Abhängigkeiten gelegt werden. Die Thematik des Auftretens von Scheinflüssen bei hohen relativen Luftfeuchten wurde bereits diskutiert. Für die entsprechenden Zeiträume wurden die berechneten Werte vollständig verworfen. Die verbleibenden Ergebnisse sind zum Teil hochgradig unsicher (vgl. Abb. 32). Deshalb wurde in Betracht gezogen, eine Selektion der Daten in Abhängigkeit vom Standardfehler vorzunehmen, um statistisch stark unsichere Werte von einer weiteren Analyse auszuschließen.

Hierbei stellt sich die Frage nach einem brauchbaren Kriterium für eine solche Selektion. Eine grundlegende Forderung könnte sein, dass ein berechneter Wert mindestens die Richtung des Partikelflusses, also mit anderen Worten das Vorzeichen der realen Depositionsgeschwindigkeit mit einer ausreichend hohen Sicherheit wiedergeben sollte. Bei einem Vertrauensintervall von $95 \%$ lässt sich daraus für die gemessene Depositionsgeschwindigkeit $\mathrm{v}_{\mathrm{d}}$ gemessen die Forderung ableiten:

$$
v_{d_{\text {gemessen }}}-1,96 \cdot E_{v_{d}} \geq 0
$$

mit: $E_{v d}$ Standardfehler der berechneten Depositionsgeschwindigkeit

Für die Größenklasse eins (Partikeldurchmesser 0,22 $\mu \mathrm{m}$ ) müssten nach diesem Kriterium $71 \%$ der Messwerte des bereits vorselektierten Datensatzes verworfen werden. Für die Klasse zwei $(0,30 \mu \mathrm{m})$ wären es $69 \%$, für Klasse drei $(0,42 \mu \mathrm{m}) 77 \%$, für Klasse vier $(0,59 \mu \mathrm{m})$ $89 \%$, und für alle anderen Klassen mehr als 95\%. Die meisten "gültigen” Messwerte erhält man erwartungsgemäß, wenn man die Konzentration über alle Klasse integriert. In diesem 
Fall müssten knapp $50 \%$ der errechneten Depositionsgeschwindigkeiten von einer weiteren Analyse ausgeschlossen werden (Abb. 37).

Abb. 37: Prozentualer Anteil zu verwerfender Messwerte der Depositionsgeschwindigkeit für die Forderung $v_{d}$ $-f^{*} E_{v_{d}} \geq 0$ für die acht kleinsten Größenklassen ( $\mathrm{f}=$ in Abhängigkeit vom gewählten Vertrauensintervall gewählter Faktor; $\mathrm{E}_{\mathrm{vd}}=$ Standardfehler der berechneten Depositionsgeschwindigkeit )

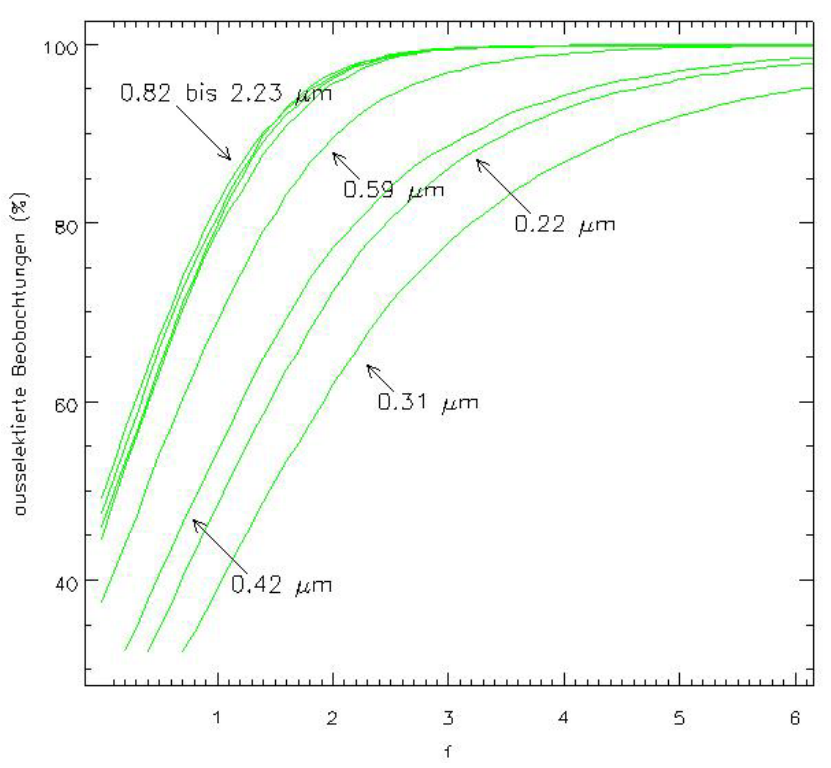

Obwohl es mit diesem Verfahren eindeutig möglich ist, statistisch zuverlässigere von statistisch weniger zuverlässigeren Messwerten zu trennen, ist eine Anwendung auf das Datenkollektiv in diesem quantitativem Ausmaße sehr kritisch. Dies kann mit folgender Überlegung gezeigt werden: Angenommen, die wahre Depositionsgeschwindigkeit $v_{d}$ sei nur von der meteorologischen Größe $x$ abhängig. Für ein bestimmtes $x$ sei $v_{d}$ konstant $5 \mathrm{~cm} / \mathrm{s}$. Der Standardfehler für die Bestimmung der Depositionsgeschwindigkeit sei als Folge des tatsächlichen Volumenstroms durch den OPC, der vorhandenen Partikelkonzentration in der Atmosphäre und der Standardabweichung der vertikalen Windgeschwindigkeit ebenfalls konstant und betrage 2,5 cm/s. Die realen Messwerte schwanken zwischen 0 und $10 \mathrm{~cm} / \mathrm{s}$. Das Kriterium für die Akzeptanz eines Messwertes sei die bereits genante Forderung, dass - bei einem Vertrauensintervall von $95 \%$ - wenigstens das Vorzeichen von $v_{d}$ (im genannten Fall also “+”) sicher bestimmt werden muss. Der Einfachheit halber sei angenommen, dass insgesamt 100 Messwerte vorliegen: 50 mit dem Wert 7,5 cm/s und 50 mit dem Wert $2,5 \mathrm{~cm} / \mathrm{s}$. Für die letzteren ist das Akzeptanzkriterium nun aber nicht erfüllt, da sie - bei dem bekannten Standardfehler von $2,5 \mathrm{~cm} / \mathrm{s}$ - sowohl negative als auch positive wahre Werte repräsentieren könnten. Diese Werte müssten also verworfen werden (nicht weil sie falsch sind, sondern weil sie besonders unsicher sind !). Der empirische Mittelwert der Messwerte für das betrachtete Beispiel ist folglich 7,5 cm/s und weicht so signifikant vom wahren Mittelwert $(5 \mathrm{~cm} / \mathrm{s}) \mathrm{ab}$. Für ein zweites $x$ sei $v_{d}$ konstant $10 \mathrm{~cm} / \mathrm{s}$, der Standardfehler bleibe unverändert. Da hier nun kein Messwert verworfen wird, erhält man empirisch nahezu den richtigen Mittelwert. Bestimmt man aus allen Messwerten die Abhängigkeit $v_{d}$ von $x$, erhält man eine unkorrekte Beschreibung des realen statistischen Zusammenhangs. 
Die praktische Relevanz dieser theoretischen Überlegung sei am Beispiel der Abhängigkeit der Depositionsgeschwindigkeit von der Schubspannungsgeschwindigkeit $u *$ gezeigt. Eine einfache lineare Abhängigkeit und eine Konstante von Null angenommen, erhält man für die ersten drei Partikelklassen die Regressionskoeffizienten 2,99, 3,02 und 4,59 (bei Angabe von $v_{d}$ in $\mathrm{cm} / \mathrm{s}$ und $u *$ in $\mathrm{m} / \mathrm{s}$; für die erste Größenklasse gilt also z.B.: $\left.v_{d}=2,99 u *\right)$. Für die Berechnung dieser Werte wurde der vorhandene Datensatz nach Forderung (41) selektiert. Die Anzahl der gültigen (hier: der statistisch sicheren) Messwerte beträgt in den drei Klassen 2606, 3611 bzw. 2177 von jeweils insgesamt 9700 berechneten Werten. Das Ergebnis ist in erster Näherung plausibel, die Depositionsgeschwindigkeit nimmt für ein gegebenes $u *$ mit der Partikelgröße zu. Betrachtet man nun die Abhängigkeit der Depositionsgeschwindigkeit von $u *$ für alle Partikel (Anzahl gültiger Werte: 4697), erhält man hier einen Regressionskoeffizienten von nur 2,38 (also: $v_{d}=2,38 u *$ ). Dies verwundert, da in den ersten drei Größenklassen ja bereits über $90 \%$ der Gesamtpartikelkonzentration vertreten sind (vgl. Tabelle 7), die Koeffizienten der Einzelklassen aber alle drei deutlich größer als 2,38 sind. Die wahrscheinlichste Erklärung für diesen Widerspruch ist, dass die Koeffizienten der Einzelklassen - entsprechend der obigen theoretischen Ausführungen - auf Grund der sehr starken Selektion von Messwerten für diese Klassen signifikant zu hoch bestimmt worden sind. Dies gilt folglich ganz besonders für den Koeffizienten der ersten $(2,99)$ und der dritten $(4,59)$ Klasse, da hier am meisten Messwerte herausselektiert wurden. Wären alle drei Koeffizienten "gleich falsch" berechnet, läge der mittlere Koeffizient $(3,02)$ mit großer Wahrscheinlichkeit deutlich zentrierter zwischen den beiden anderen als er dies jetzt tut.

Der Ansatz, den vorhandenen Datensatz nach Forderung (41) zu selektieren, musste deshalb wieder verworfen werden. Um dennoch eine Verbesserung der Datenqualität zu erreichen, wurde der Datensatz in drei Schritten leicht modifiziert:

1. Aus den vorhandenen Daten wurden 90 Tage ausgewählt, für die ein vollständiger oder nahezu vollständiger Tagesgang der Depositionsgeschwindigkeit berechnet werden konnte (also keine größeren Ausfälle infolge von Nebelereignissen und Regen auftraten).

2. Die berechneten Depositionsgeschwindigkeiten wurden über ein gleitendes Mittel leicht geglättet, um die hohe statistische Unsicherheit der Einzelwerte etwas auszugleichen.

3. Negative Depositionsgeschwindigkeiten wurden verworfen. Da es sich fast ausschließlich um Einzelwerte handelt, wurden sie als Ausreißer betrachtet. Es wurde angenommen, dass in diesen Fällen unbekannte Ursachen zu fehlerhaften Werten geführt haben.

Für den in dieser Art modifizierten Datensatz wurde wiederum die einfache lineare Abhängigkeit von $v_{d}$ und $u *$ geprüft. Der Regressionskoeffizient fällt nun für die ersten drei Größenklassen mit 1,93, 2,27 bzw. 2,73 deutlich niedriger als für die ursprünglich nach Forderung (41) selektierten Daten. Der Koeffizient für alle Klassen beträgt 2,11 und liegt damit zwischen dem Koeffizienten der ersten und zweiten Größenklasse. Dieses Ergebnis ist plausibel. 

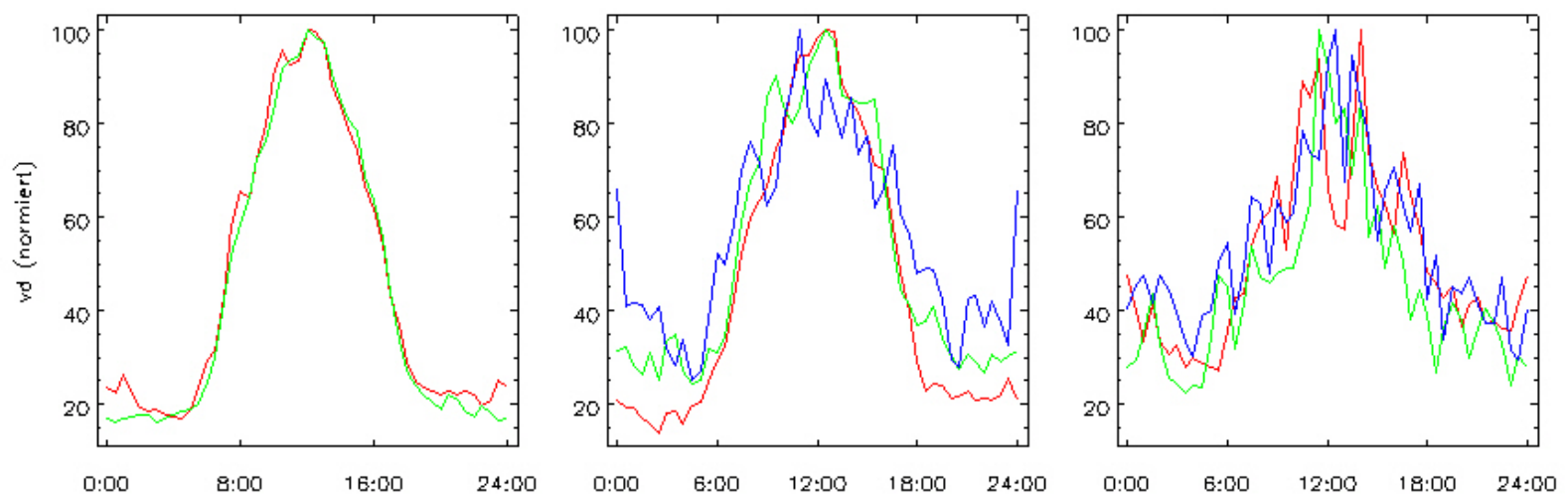

Abb. 38: Mittlere Tagesgänge der Depositionsgeschwindigkeit für die 8 kleinsten Klassen des OPC. Wie Abb. 28, jedoch nach Modifizierung des Datensatzes (Erläuterung siehe Text)

In Abb. 38 wird gezeigt, dass mit der Modifizierung des Datensatzes tatsächlich eine Verbesserung der Qualität des Datensatzes erreicht werden konnte. Für alle Größenklassen ist jetzt ein akzeptabler mittlerer Tagesgang der berechneten Depositionsgeschwindigkeit zu erkennen. Allerdings steigen die Nachtwerte beginnend mit Kanal vier auf durchschnittlich $40 \%$ des Tagesmaximums an, was unrealistisch hoch ist. Dieser Effekt muss wahrscheinlich auf die noch immer hohe statistische Unsicherheit in diesen Klassen und das damit häufige Auftreten von negativen Werten zurückgeführt werden, die dann im Zuge der Modifizierung verworfen wurden.

\subsubsection{Mittlere Beträge und Abhängigkeiten von meteorologischen Größen}

Auf der Basis des modifizierten Datensatzes sollen nun die statistische Abhängigkeiten der gemessenen Depositionsgeschwindigkeit von meteorologischen Parametern detaillierter untersucht werden. Dabei wurden alle erfassten Parameter geprüft, insbesondere die horizontale Windgeschwindigkeit $u$, der fühlbare Wärmefluss $H$, die Schubspannungsgeschwindigkeit $u *$, die turbulente Energie $E$ und die Monin-Obuchov-Mischungslänge $L$ (Tabelle 10). Es wurde zwischen stabiler $(L>0)$ und labiler $(\mathrm{L}<0)$ Luftschichtung unterschieden.

Die stärkste Korrelation tritt für alle Partikelklassen mit bis zu $67 \%$ bei stabiler Schichtung mit der Schubspannungsgeschwindigkeit $u *$ bzw. der turbulenten Energie $E$ auf. Bei labilem Verhältnissen ist die Korrelation mit beiden Größen deutlich geringer und kann mit etwa $35 \%$ veranschlagt werden (für $u *$ vgl. Abb. 39). Die Korrelation mit der horizontalen Windgeschwindigkeit $u$ fällt um jeweils etwa $20 \%$ niedriger aus, ist also zur Parametrisierung der Depositionsgeschwindigkeit etwas schlechter geeignet als $u *$ oder $E$. Mit dem fühlbaren Wärmefluss kann ein statistischer Zusammenhang erwartungsgemäß vor allem für labile Schichtungen gezeigt werden, er beträgt dann maximal $49 \%$. Ein linearer statistischer $\mathrm{Zu}-$ sammenhang mit der Monin-Obuchov-Mischungslänge $L$ selbst kann weder für stabile noch für labile Schichtung ausgemacht werden. Generell nimmt die Korrelation zwischen berech- 
neter Depositionsgeschwindigkeit und den meteorologischen Größen mit zunehmender Partikelgröße - und damit mit geringeren Partikelkonzentrationen und zunehmender statistischer Unsicherheit der Einzelwerte - ab.

Tabelle 10: Lineare Korrelationskoeffizienten der berechneten Depositionsgeschwindigkeiten $v_{D}$ mit gemessenen meteorologischen Parametern (Windgeschwindigkeit $u$, fühlbarer Wärmefluss $H$, Schubspannungsgeschwindigkeit $u *$, turbulente Energie $E$ und Monin-Obuchov-Mischungslänge $L$ ) sowie der Anzahl $n$ der untersuchten Halbstundenwerte für die acht kleinsten Partikelgrößenklassen. Der erste Wert jeweils für stabile, der zweite für labile Schichtung der Atmosphäre

\begin{tabular}{|c|c|c|c|c|c|c|c|c|c|c|c|c|}
\hline \multirow{2}{*}{$\begin{array}{c}\text { Klasse } \\
1\end{array}$} & \multicolumn{2}{|r|}{$u$} & \multicolumn{2}{|c|}{$H$} & \multicolumn{2}{|c|}{$u^{*}$} & \multicolumn{2}{|c|}{$E$} & \multicolumn{2}{|c|}{$L^{-1}$} & \multicolumn{2}{|c|}{$n$} \\
\hline & 0,46 & 0,18 & $-0,15$ & 0,44 & 0,62 & 0,34 & 0,64 & 0,34 & $-0,11$ & 0,03 & 1409 & 1528 \\
\hline 2 & 0,41 & 0,18 & $-0,11$ & 0,49 & 0,61 & 0,36 & 0,61 & 0,36 & $-0,12$ & 0,04 & 1498 & 1540 \\
\hline 3 & 0,35 & 0,17 & $-0,16$ & 0,41 & 0,52 & 0,32 & 0,50 & 0,33 & $-0,10$ & 0,02 & 1367 & 1481 \\
\hline 4 & 0,41 & 0,18 & $-0,16$ & 0,38 & 0,52 & 0,30 & 0,51 & 0,32 & $-0,09$ & 0,03 & 1116 & 1307 \\
\hline 5 & 0,32 & 0,26 & $-0,17$ & 0,21 & 0,42 & 0,34 & 0,39 & 0,33 & $-0,09$ & $-0,04$ & 869 & 1017 \\
\hline 6 & 0,37 & 0,27 & $-0,20$ & 0,15 & 0,45 & 0,31 & 0,44 & 0,30 & $-0,14$ & $-0,12$ & 913 & 896 \\
\hline 7 & 0,27 & 0,25 & $-0,24$ & 0,12 & 0,35 & 0,29 & 0,30 & 0,27 & $-0,10$ & $-0,04$ & 859 & 774 \\
\hline 8 & 0,26 & 0,19 & $-0,24$ & 0,15 & 0,34 & 0,23 & 0,30 & 0,21 & $-0,08$ & 0,04 & 756 & 605 \\
\hline \multirow[t]{2}{*}{ alle } & 0,47 & 0,18 & $-0,14$ & 0,49 & 0,66 & 0,35 & 0,67 & 0,36 & $-0,12$ & 0,03 & 1505 & 1539 \\
\hline & stabil & labil & stabil & labil & stabil & labil & stabil & labil & stabil & labil & stabil & labil \\
\hline
\end{tabular}

Abb. 39: Depositionsgeschwindigkeit (alle Klassen) als Funktion von $u *$ für labile (rot) und stabile (grün) Schichtung

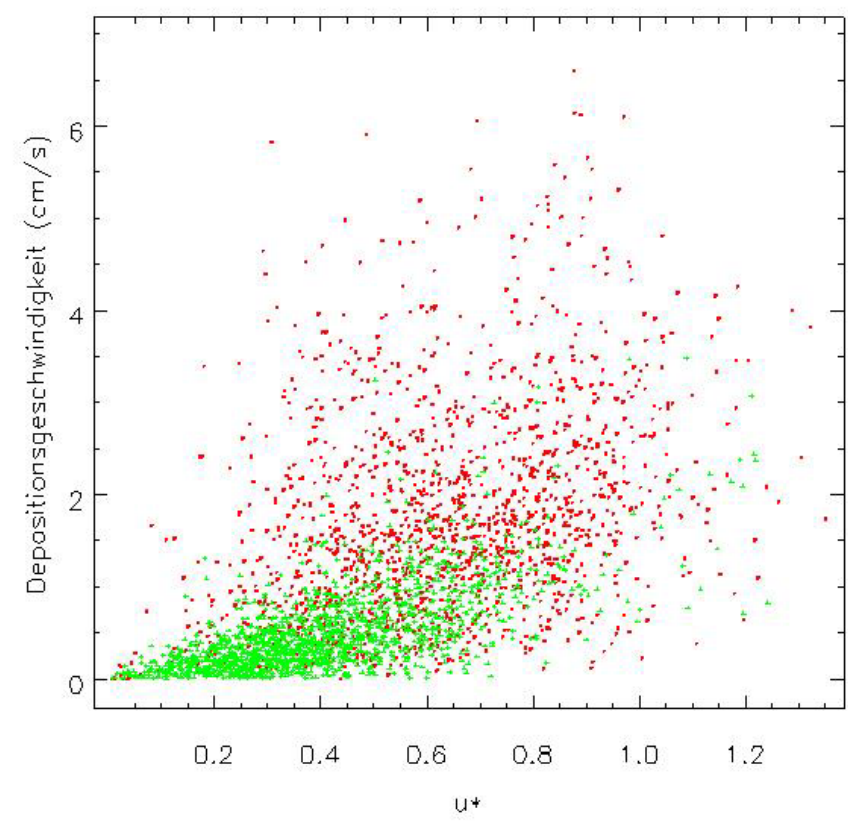

Die starke Abhängigkeit der Depositionsgeschwindigkeit $v_{d}$ von $u *$ deutet darauf hin, dass die Prozesse der Trockenen Deposition luftgetragener Partikel (vgl. Kapitel 1.3.2.1) in deutlicher 
Weise von der Ausbildung der mechanisch induzierten Turbulenz abhängig sind. Betrachtet man diese Abhängigkeit bei neutralen Schichtung der Atmosphäre, so kann der Zusammenhang zwischen $v_{d}$ von $u_{*}$ - insbesondere für $u *>0,3$ und $u_{*}<0,9 \mathrm{~m} / \mathrm{s}-$ in sehr befriedigender Weise als Funktion von $u_{*}{ }^{2}$ und des Partikeldurchmessers dargestellt werden (Abb. 40).

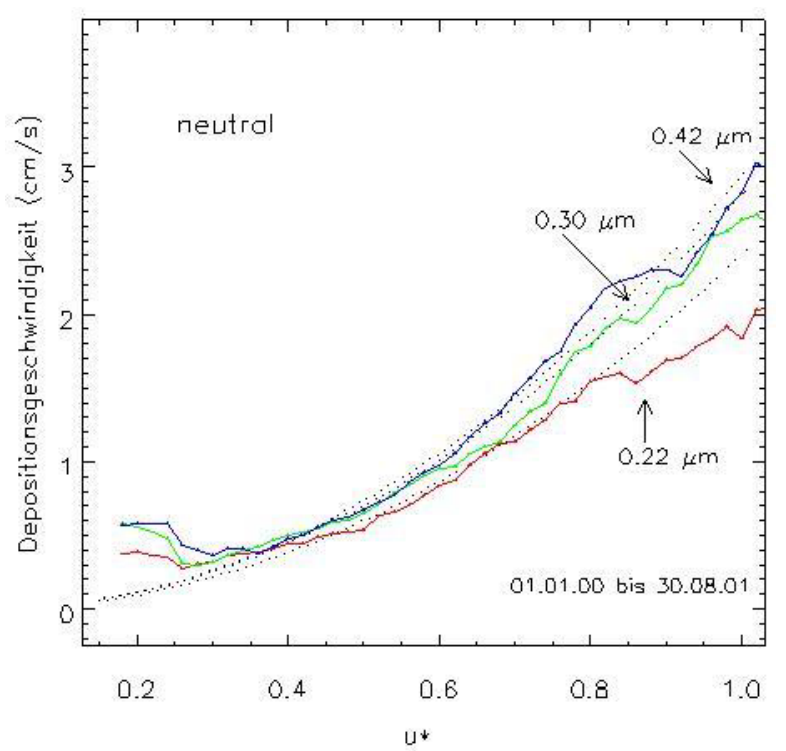

Abb. 40: Depositionsgeschwindigkeit der drei kleinsten Partikelklassen als Funktion von $u *$ für neutrale Schichtung (Medianwerte, Kurven geglättet, rot: $0,22 \mu \mathrm{m}$, grün: $0,30 \mu \mathrm{m}$, blau: $0,42 \mu \mathrm{m}$ Partikeldurchmesser). Die gepunkteten Linien stellen den Verlauf der Funktion $f \cdot u_{*}^{2}$ mit $f=2,40,2,80$ bzw. 2,94 dar, mit der die Abhängigkeit zwischen $u *$ und der Depositionsgeschwindigkeit bei neutraler Schichtung gut beschrieben wird.

Dieser Zusammenhang wird bei labiler und stabiler Schichtung der Atmosphäre unter dem Einfluss thermisch induzierter Vorgänge modifiziert. Insbesondere für $0,1 \mathrm{~m} / \mathrm{s}<u *<0,6 \mathrm{~m} / \mathrm{s}$ wird die Partikeldeposition unter labilen Verhältnissen deutlich erhöht, während die Deposition umgekehrt unter stabilen Verhältnissen für $0,4 \mathrm{~m} / \mathrm{s}<u *<0,9 \mathrm{~m} / \mathrm{s}$ fast ebenso deutlich reduziert wird (Abb. 41). Letztere Beobachtung erklärt auch die negativen Korrelationskoeffizienten zwischen der Depositionsgeschwindigkeit und dem Reziprokwert der MoninObuchov-Mischungslänge bei stabiler Schichtung in Tabelle 10: je stärker eine stabile Schichtung in der Atmosphäre ausgeprägt ist, umso stärker wird ein Partikeleintrag in den betrachteten Fichtenforst unterdrückt.

In Abb. 41 ist zu erkennen, dass die Partikeldeposition bei gleichen Randbedingungen (hier: bei gleichen $u *)$ mit der Partikelgröße zunimmt. Für die vierte Größenklasse kann diese Aussage jedoch leider bereits nicht mehr eindeutig gezeigt werden. Offensichtlich ist es also auch mit der Modifizierung des Datensatz nicht gelungen, die Unzulänglichkeiten und Unsicherheiten für die Resultate der Klassen 0,59 $\mu \mathrm{m}$ (und größer) vollständig zu beseitigen. Dementsprechend müssen die ermittelten mittleren Depositionsgeschwindigkeiten für diese Klassen mit Vorsicht betrachtet werden (Tabelle 11). Für die drei kleinsten Größenklassen zeigt die gute Übereinstimmung der Kurven in Abb. 41 dagegen, dass hier die ermittelten Depositionsgeschwindigkeiten offensichtlich sehr zuverlässig die realen Verhältnisse wiederspiegeln. Die über den gesamten Messzeitraum gemittelten Depositions- 
geschwindigkeiten betragen für diese drei Klassen $1,05 \mathrm{~cm} / \mathrm{s}(0,22 \mu \mathrm{m}), 1,23 \mathrm{~cm} / \mathrm{s}(0,30 \mu \mathrm{m})$ bzw. $1,47 \mathrm{~cm} / \mathrm{s}(0,42 \mu \mathrm{m}$ Partikeldurchmesser $)$.
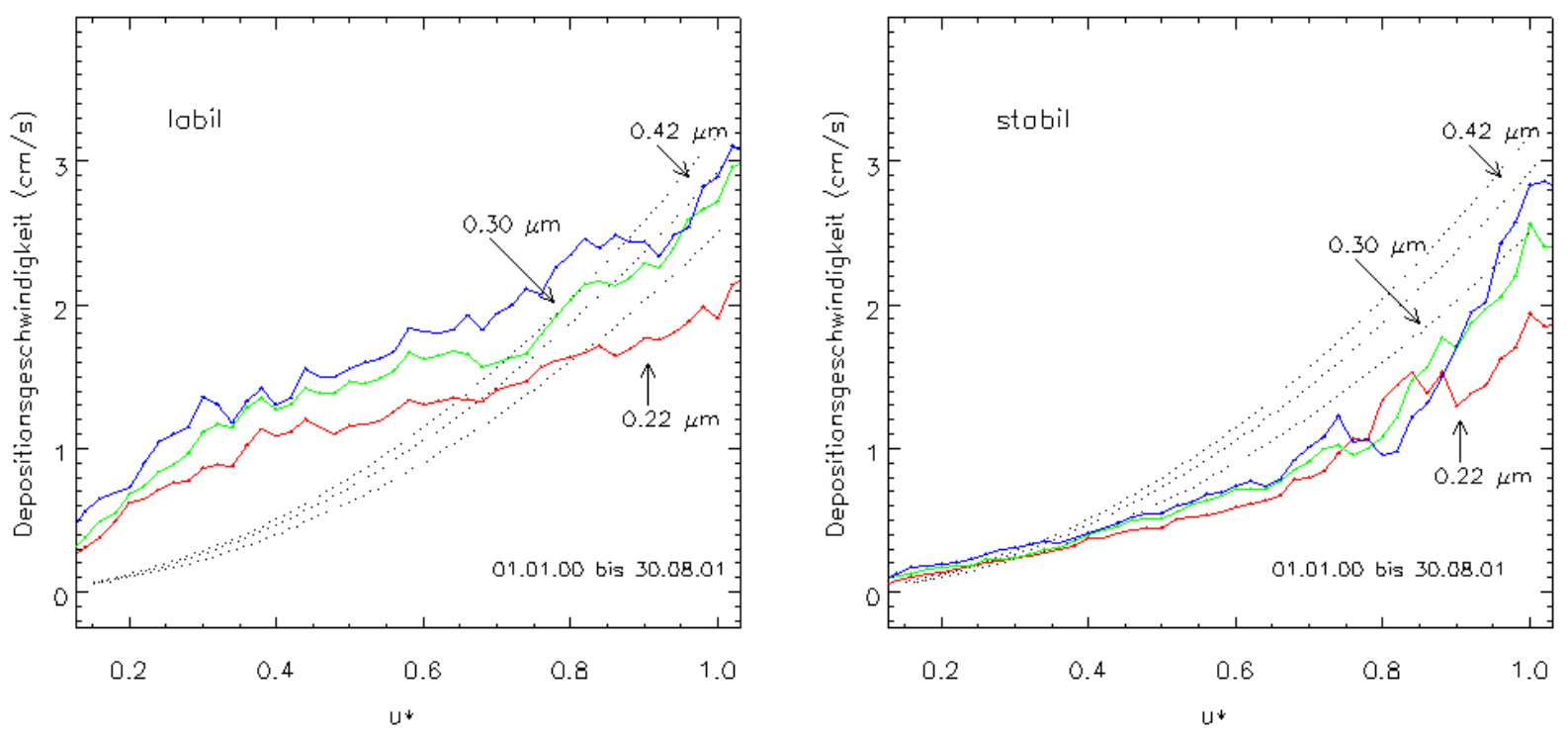

Abb. 41: Depositionsgeschwindigkeit der drei kleinsten Partikelklassen als Funktion von $u *$ für labile (links) und stabile (rechts) Schichtung (Medianwerte, Kurven geglättet, rot: 0,22 $\mu \mathrm{m}$, grün: 0,30 $\mu \mathrm{m}$, blau: 0,42 $\mu \mathrm{m}$ Partikeldurchmesser). Gepunktet: idealisierter Verlauf der Abhängigkeit der Depositionsgeschwindigkeit von $u *$ bei neutraler Schichtung (vgl. Abb. 40)

Tabelle 11: Mittlere Depositionsgeschwindigkeiten für die 8 kleinsten Klassen des OPC (alle Beobachtungen des modifizierter Datensatzes, Erläuterungen siehe Text)

\begin{tabular}{|c|c|c|}
\hline Klasse & $\begin{array}{c}\text { mittlerer } \\
\text { Durchmesser } \\
(\mu \mathrm{m})\end{array}$ & $\begin{array}{c}\text { mittlere Depositions- } \\
\text { geschwindigkeit } \\
(\mathrm{cm} / \mathrm{s})\end{array}$ \\
\hline \hline 1 & 0,22 & 1,05 \\
\hline 2 & 0,30 & 1,23 \\
\hline 3 & 0,42 & 1,47 \\
\hline 4 & 0,59 & $1,72^{\text {a) }}$ \\
\hline 5 & 0,82 & $2,21^{\text {a) }}$ \\
\hline 6 & 1,14 & $3,11^{\text {a) }}$ \\
\hline 7 & 1,60 & $5,00^{\text {a) }}$ \\
\hline 8 & 2,23 & $10,01^{\text {a) }}$ \\
\hline alle & & 1,15 \\
\hline
\end{tabular}

a) als unsicher einzustufende Werte 
Resultate der eigenen Messungen

\subsection{Messungen mit dem CPC}

Wie in Kapitel 3.2.3 erläutert, wurde der Partikelzähler CPC 3022 der Firma TSI (hiernach: CPC) zeitweilig parallel zum OPC für die Bestimmung der vertikalen Partikelflusse mit Hilfe der Eddy - Kovarianzmethode eingesetzt, und zwar sowohl als einzelner Partikelzähler ohne Größen-Fraktionierer als auch in Kombination mit einem Größen-Fraktionierer 3071. Die Kovarianzmessungen mit dem CPC ohne Größen-Fraktionierer verliefen im Vergleich zu den bereits beschriebenen Messungen mit dem OPC relativ unproblematisch: das Konzentrationssignal ist nur wenig verrauscht, und die Depositionsgeschwindigkeit kann im allgemeinen mit einer etwas höheren statistischen Sicherheit als beim OPC bestimmt werden. Da diese Messungen jedoch nicht größenspezifisch sind, müssen die gefundenen Beträge einem relativ breiten Größenbereich luftgetragener Partikel zugeordnet werden. Der mittlere Durchmesser dieses Größenbereichs kann mit etwa 0,02 $\mu \mathrm{m}$ Durchmesser angenommen werden (Abb. 14), die mittlere Depositionsgeschwindigkeit für diesen Bereich wurde mit $0,77 \mathrm{~cm} / \mathrm{s}$ bestimmt (Messzeitraum: 3 Wochen). Die gefundenen Ergebnisse sind insbesondere auch in Hinblick auf die Ergebnisse mit dem OPC - System sehr plausibel (Abb. 42, links; vgl. auch Abb. 43).
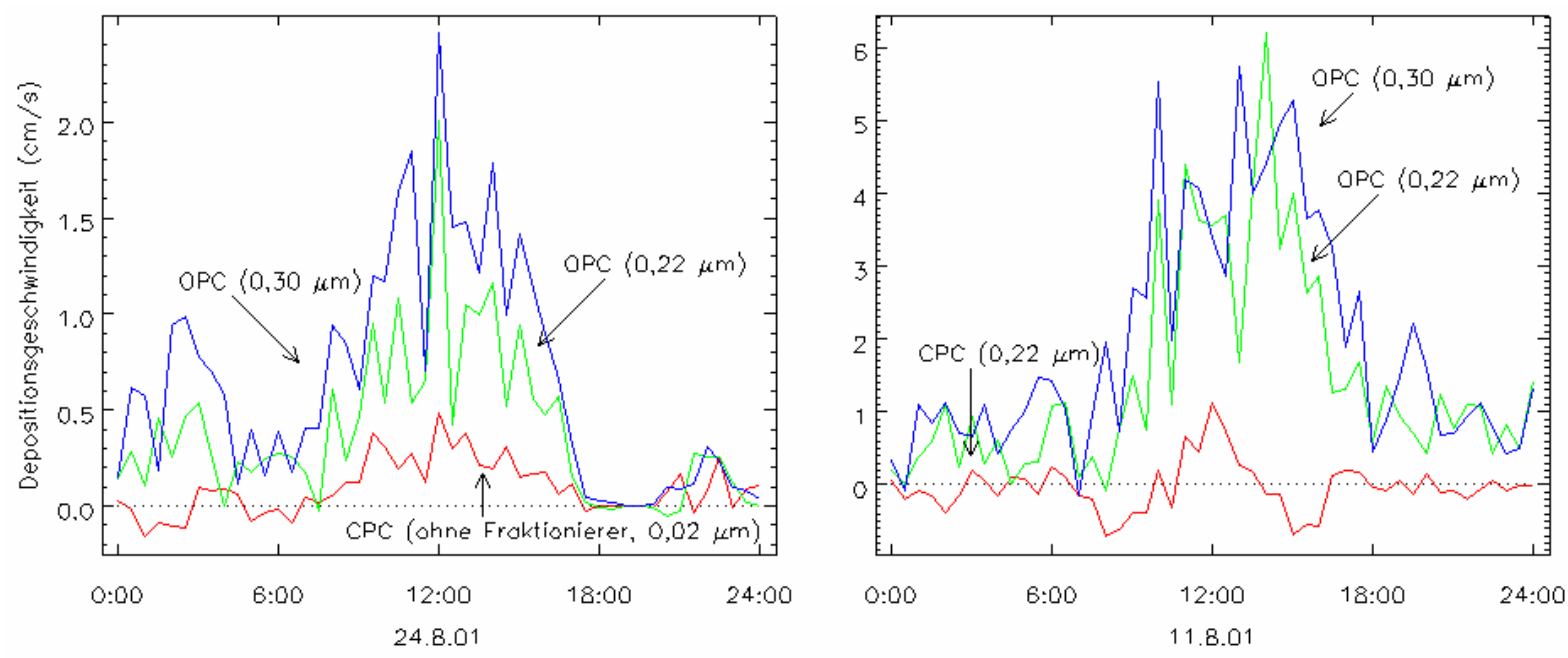

Abb. 42: Tagesgänge der Depositionsgeschwindigkeit am 24. und 11.8.01, bestimmt mit dem CPC ohne Größen-Fraktionierer (24.8.) bzw. mit Fraktionierer (11.8., Partikeldurchmesser 0,22 $\mu \mathrm{m}$ ) im Vergleich mit den Ergebnissen des OPC - Systems (Größenklassen 0,22 und 0,3 $\mu$ m Durchmesser).

Die durchgeführten Messungen mit dem CPC in Kombination mit dem Größen-Fraktionierer TSI 3071 führen dagegen zu keinem befriedigenden Ergebnis. Die berechneten Kovarianzen sind sehr klein und zeigen selbst an Tagen, an denen durch das OPC - System ein ausgesprochen deutlicher Tagesgang der Depositionsgeschwindigkeit bestimmt wurde, nur ansatzweise einen solchen Gang im Tagesverlauf. Insbesondere stimmen die berechneten Werte bei gleichen betrachteten Partikelgrößen nicht mit den Ergebnisse des OPC - Systems überein (Abb. 42, rechts). Es muss deshalb festgestellt werden, dass mit der Kombination aus TSI 3022 und TSI 3071 keine sinnvollen Eddy - Kovarianzmessungen möglich sind. 


\section{Diskussion}

In diesem Kapitel sollen die Ergebnisse der vorliegenden Arbeit diskutiert und interpretiert werden. Zunächst werden dabei methodologische Probleme besprochen, anschließend werden einige verschiedene Einzelaspekte der gewonnenen Resultate erörtert. Ein Ausblick auf die zukünftige Bedeutung der Eddy - Kovarianzmethode für Depositionsmessungen luftgetragener Partikel schließt das Kapitel ab.

\subsection{Methodologische Probleme}

\subsubsection{Der PCS-2000 als meteorologisches Messgerät: Probleme und potentielle Einsatzmöglichkeiten}

Mit der vorliegenden Arbeit ist das erste Mal der Versuch unternommen worden, mit dem kommerziell erhältlichen Einzelpartikelzähler PCS-2000 der Firma Palas (hiernach: OPC) Untersuchungen zum Vertikaltransport luftgetragener Partikel durchzuführen. Für eine allgemeine Bewertung der Einsatzmöglichkeiten dieses Gerätes im Rahmen meteorologischer Messungen ist zunächst festzustellen, dass es sich beim OPC um ein hochwertiges und robustes Messgerät handelt, das vor allem bei Untersuchungen industrieller Prozesse wie z.B. Filtertests, Abgasuntersuchungen u.ä. zum Einsatz kommt. Hier treten allerdings im Vergleich zum atmosphärischen Aerosol im allgemeinen relative hohe Partikelkonzentrationen (mehr als 10000 Partikel pro $\mathrm{cm}^{3}$ ) und auch größere Partikeldurchmesser auf. Für diese Bedingungen ist der OPC entwickelt und optimiert worden, und unter diesen Bedingungen wird er mit gutem Erfolg eingesetzt.

Im Zuge der vorliegenden Arbeit konnten die ohne Frage großen Potentiale der Geräts jedoch kaum genutzt werden. Vielmehr haben Limitationen des OPC, wie sie sich aus der begrenzten Durchflussmenge und dem detektierbaren Größenspektrum ergeben, eine umfangreiche und eindeutige Interpretation der durchgeführten Messungen merklich eingeschränkt. Von Beginn der Messungen an ist bekannt gewesen, dass der OPC auf Grund der verwendeten Weißlichtquelle nur einen Teil des interessierenden Größenspektrums atmosphärischer Partikel abdecken kann. Im Zuge der Messungen wurden weitere Einschränkungen gefunden. Hier muss der ungenügend niedrige Zählwirkungsgrad in den kleinsten Partikelklasse ebenso genannt werden, wie die Tatsache, dass für die meisten Partikelklassen die Konzentrationen am Untersuchungsort zu niedrig sind, um das Konzentrationssignal bei einer Messfrequenz von $1 \mathrm{~Hz}$ mit einer befriedigenden statistischen Sicherheit aufnehmen und damit die vertikalen Partikelflüsse zuverlässig berechnen zu können. 
Schon JAENICKE (1993) führt aus, dass kommerziell angebotene optische Partikelanalysatoren für luftchemische und mikrometeorologische Untersuchungen häufig unter Bedingungen betrieben werden müssen, für die sie letztlich nicht geeignet sind. Hieraus folgt unmittelbar die Forderung nach Eigenentwicklungen von Messgeräten für wissenschaftliche Untersuchungen, wie sie immer wieder von Aerosolforschern vorgetragen worden ist. Für die Bestimmung der Depositionsgeschwindigkeit luftgetragener Partikel mit Hilfe der Eddy Kovarianzmethode, die in dieser Arbeit versucht wurde, werden Partikelzähler benötigt, die den Größenbereich atmosphärischer Partikel in wesentlichen Anteilen und damit vor allem auch für Partikel kleiner $<0,2 \mu \mathrm{m}$ Durchmesser abdecken, und die gleichzeitig eine hohe statistische Sicherheit bei der Bestimmung der Partikelkonzentration auch für hochfrequente Messungen realisieren können. Diese Forderungen kann der verwendete OPC - einerseits auf Grund der verwendeten Weißlichtquelle und andererseits wegen des zugrunde liegenden Konzepts der Einzelpartikelanalyse - definitiv nur teilweise erfüllen. Bezüglich der statistischen Sicherheit muss dabei klar benannt werden, dass die Analyse des einzelnen Teilchens zwar prinzipiell ein sehr genaues Messverfahren ist, das aber gleichzeitig - in Folge des bei schnellen Messungen zwangsläufig kleinen Stichprobenumfangs - unter Umständen hochgradig unsichere Mittelwerte für kleine Zeitschritte (eine Sekunde und kürzer) liefern muss. Insgesamt ist deshalb festzuhalten, dass der OPC für zeitkritische Messungen, wie sie im Rahmen der vorliegenden Arbeit durchgeführt wurden, lediglich eingeschränkt geeignet ist.

Die vorgelegten Untersuchungen zeigen aber auch, dass die kontinuierliche Messung der Konzentration atmosphärischer Partikel im Größenbereich zwischen 0,2 und $10 \mu \mathrm{m}$ Durchmesser eine hervorragende Möglichkeit darstellt, Nebelbildung zu analysieren sowie Nebelperioden quantitativ und qualitativ zu dokumentieren. Eine alternative Untersuchung dieser Prozesse und Ereignisse ist zum gegenwärtigen Zeitpunkt am Institut für Bioklimatologie (IBK) der Universität Göttingen nicht realisierbar, so dass es sich anbietet, diese potentielle Einsatzmöglichkeit zukünftig für den $\mathrm{OPC} \mathrm{zu}$ überprüfen. Hierfür wäre es notwendig, Vergleichsmessungen mit einem konventionellen Nebelsichtgerät durchzuführen sowie Messvorgang und Auswertung zu standardisieren. Im Rahmen der Möglichkeiten dieser Arbeit konnte lediglich die gute Plausibilität von Aussagen zu Nebelereignissen aus OPC Messungen festgestellt werden. Da hierzu Aufnahmen einer installierten digitalen Kamera verwendet wurden, waren die Plausibilitätsprüfungen allerdings per se auf Tageszeiten zwischen Sonnenauf- und -untergang beschränkt.

\subsubsection{Notwendige Randbedingungen für die Quantifizierung der Partikeldeposition mit Hilfe der Eddy - Kovarianzmethode}

Die Eddy - Kovarianzmethode ist eine in der Mikrometeorologie weit verbreitete Methode zur Bestimmung vertikaler Energie- und Stofftransporte. Insbesondere sind auch die Anforderungen an die Methode und die Begrenzungen ihrer Einsatzmöglichkeiten gut bekannt. In Verbindung mir der vorliegenden Arbeit hat sich jedoch gezeigt, dass einer der wesentlichen 
Grundvoraussetzungen der Eddy - Kovarianzmethode nicht immer genügend Augenmerk geschenkt wird. Es ist die Tatsache, dass es für eine zuverlässige Berechnung der vertikalen Stoffflüsse nicht ausreicht, alle am turbulenten Transport beteiligten Fluktuationen (nur) mit einer ausreichend hohen zeitlichen Auflösung zu erfassen. Vielmehr ist es notwendig, die vorhanden Fluktuationen auch mit einer hinreichend genauen statistischen Sicherheit zu bestimmen. Diese simple klingende Forderung wird heute von vielen meteorologischen Messsystemen, Sensoren und Gasanalysatoren bereits wie selbstverständlich erfüllt. Leider bedeutet das aber nicht, dass man dies deshalb auch generell für alle in der Mikrometeorologie zum Einsatz kommenden Analysatoren per se vorauszusetzen darf. Insbesondere kann auch das Verfahren der Einzelpartikelanalyse, wie es in zahlreichen optischen Partikelzählern und so auch in dem in der vorliegenden Arbeit verwendeten OPC angewendet wird, eine solche statistische Sicherheit für hochfrequente, größenspezifische Messungen der Partikelkonzentration bei den üblicherweise in der Troposphäre vorkommenden Teilchendichten nicht leisten.

Wie gezeigt wurde, hat die statistische Unsicherheit bei der Konzentrationsbestimmung wiederum eine hohe statistische Unsicherheit der Beträge zur Folge, die mit Hilfe der Eddy Kovarianzmethode aus den gemessenen Konzentrationsreihen für die Depositionsgeschwindigkeit luftgetragener Partikel berechnet werden. Diese Unsicherheit ist in der Literatur bereits mehrfach beschrieben und diskutiert worden. So finden GALLAGHER ET AL. (1997) bei ähnlichen Untersuchungen einen Standardfehler von 30 bis $50 \%$ der bestimmten Depositionsgeschwindigkeit für Messungen am Tage, während die Fehler in der Nacht die Beträge der Messwerte sogar um ein Vielfaches übersteigen. Dies entspricht sehr genau den Resultaten der vorliegenden Arbeit. Auch konstatieren GALLAGHER ET AL. (1997) eine Zunahme des Standardfehlers mit der Partikelgröße, so dass sie - ebenfalls in Übereinstimmung mit der vorliegenden Arbeit - Partikel mit einem Durchmesser $>0,5 \mu \mathrm{m}$ von einer Analyse im Sinne der Eddy - Kovarianzmethode ausschließen müssen. NEUMANN \& HARTOG (1985) berichten von großen Standardfehlern über die gesamte Messzeit. Diese waren häufig größer als die berechneten Werte selbst, wobei die größten Fehler ebenfalls bei Partikeln mit einem Durchmesser $>0,5 \mu \mathrm{m}$ auftraten. In einer vergleichenden Aufstellung der Ergebnisse dreizehn verschiedener Untersuchungstechniken zur Partikeldeposition im Speulder Forest (Niederlande) bewerten ERISMAN ET AL. (1997b) die Kovarianzmethode für Partikel zwischen 0,1 und $3 \mu \mathrm{m}$ Durchmesser mit einem zufälligen Fehler zwischen 50 und $150 \%$ als statistisch deutlich unsicherer als alle anderen eingesetzten Verfahren. Hierbei darf freilich nicht übersehen werden, dass letztere im Gegensatz zur Kovarianzmethode oft hohe systematische Unzulänglichkeiten aufweisen (vgl. Kapitel 1.3.2.3).

Im Zuge der vorliegenden Arbeit konnte gezeigt werden, dass die statistische Unsicherheit bei der Partikelflussbestimmung mit Hilfe der Eddy - Kovarianzmethode aus der Kombination der Standardabweichung der vertikalen Windgeschwindigkeit, der mittleren Partikelkonzentration in den einzelnen Partikelklassen, des pro Zeiteinheit untersuchten Volumens als gerätespezifische Eigenschaft und der benötigten Messfrequenz sehr genau vorausgesagt 
werden kann. Alle diese Größen sind für einen bestimmten Messort bzw. ein spezielles Messgerät entweder bekannt oder können ungefähr abgeschätzt werden. Damit ist die Möglichkeit gegeben, für zukünftige Messungen die unvermeidliche, aber doch eben vorhersagbare Unsicherheit der Messungen im voraus zu bestimmen, mit den Erwartungswerten $\mathrm{zu}$ vergleichen und hieraus wiederum ggf. Konsequenzen für die geplante Untersuchung beispielsweise bezüglich der Geräteauswahl oder bezüglich einer Gerätemodifikation zu berücksichtigen.

Buzorius et AL. (1998) haben das Problem der statistischen Sicherheit durch den Einsatz eines Kondensationskernzählers für die Bestimmung der Partikelkonzentration gelöst. Da es sich hierbei um ein Verfahren handelt, bei dem die Partikelkonzentration am gesamten Partikelensemble ermittelt wird (vgl. Kapitel 1, Abschnitt Messtechnische Erfassung von Partikeln), ist das Volumen der Probenluft, das innerhalb eines Zeitintervalls auf das Vorhandensein von Partikeln untersucht wird, prinzipiell deutlich größer und damit auch die statistische Sicherheit bei der Konzentrationsbestimmung deutlich höher als bei der Einzelpartikelanalyse. Die Autoren benutzen einen TSI 3010 der Firma TSI, so dass ihnen ein Probevolumen von $17 \mathrm{~cm}^{3}$ Luft pro Sekunde zur Verfügung steht. Ausgehend von Konzentrationen von etwa 1000 Teilchen pro $\mathrm{cm}^{3}$ Luft betonen die Autoren ausdrücklich die Notwendigkeit eines solch großen Probevolumens für eine erfolgreiche Bestimmung von Partikelflüssen mit Hilfe der Eddy - Kovarianzmethode ${ }^{18}$.

Auch LAMAUd ET AL. (1994a) gelingt es, die Konzentration luftgetragener Partikel zeitlich hochaufgelöst mit einer hohen statistischen Sicherheit aufzuzeichnen. Die von diesen Autoren gezeigten Power- und Kospektren für die Partikelkonzentration zeigen über den gesamten Frequenzbereich die gleiche universelle Form wie für die anderen skalaren Eigenschaften der Atmosphäre auch (hier: Windgeschwindigkeit, Lufttemperatur, Ozon- und Kohlendioxidkonzentration). Die Autoren benutzen für ihre Untersuchungen einen selbstentwickelten Partikelzähler, in dem vorhandene Teilchen in der Luft elektrisch aufgeladen werden und der resultierende Strom als Maß für die Anzahldichte gemessen wird. Der realisierte Volumenfluss ist mit 2,5 1/s sogar noch höher als bei BUZORIUS ET AL. (1998).

Mit dem in der vorliegenden Arbeit eingesetztem OPC können dagegen selbst in der modifizierten Version des Gerätes lediglich $0,05 \mathrm{~cm}^{3}$ Luft pro Sekunde auf das Vorhandensein von Partikeln untersucht werden. Es liegt auf der Hand, dass deshalb - in Übereinstimmung mit ähnlichen Arbeiten, bei denen optische Einzelpartikelzähler für die Eddy - Kovarianzmethode eingesetzt wurden - das Problem eines hohen statistischen Fehlers der Messergebnisse weitaus stärker in das Gewicht fällt als bei den erwähnten Arbeiten von BUZORIUS ET AL. (1998) und LAMAUD ET AL. (1994a). Die Entscheidung für den Einsatz des OPC begründet sich darin, dass die Nutzung des OPC größenspezifische Aussagen zur Partikelkonzentration

\footnotetext{
${ }^{18}$ Nach Meinung der Autoren sind sogar Kondensationskernzähler mit geringerem Volumendurchfluss als der TSI 3010, wie z.B. der in der vorliegenden Arbeit probeweise zum Einsatz gekommene TSI 3022, bereits nicht mehr für den Einsatz schneller Konzentrationsmessungen geeignet.
} 
und damit auch zur Partikeldeposition ermöglicht. Bei den von den vorgenannten Autoren verwendeten Geräten sind größenspezifische Untersuchungen zur Partikeldeposition dagegen nicht (oder nur unter gewissen Bedingungen - vgl. nächster Absatz) möglich. Eine größenspezifische Untersuchung aber war das Ziel der vorliegenden Arbeit. Die Motivation hierfür ergibt sich aus Beobachtungen wie z.B. von GALLAGHER ET AL. (1997), die zeigen, dass zu einem bestimmten Zeitpunkt Partikel einer bestimmten Größe auf der Erdoberfläche deponiert werden können, während etwas größere oder etwas kleinere Partikel gleichzeitig in die Atmosphäre emittiert werden. Bei einer integrativen, nichtgrößenspezifischen Betrachtung können solche Situationen nicht realistisch abgebildet werden. Deshalb sollte und musste die vorliegende Arbeit über den Ansatz von BUZORIUS ET AL. (1998) und LAMAUD ET AL. (1994a), die Partikelkonzentration und -deposition lediglich als integrative Größe zu bestimmen, hinausgehen.

Theoretisch bietet die Kombination mit einen elektrostatischen Größen-Fraktionierer die Möglichkeit, größenspezifische Untersuchungen der Partikeldeposition mit Hilfe der Eddy Kovarianzmethode auch mit einem Kondensationskernzählers durchzuführen. In der vorliegenden Arbeit wurde versucht, diesen Ansatz mit Hilfe eines zur Verfügung stehenden elektrostatischen Größen-Fraktionierers TSI 3071 und Kondensationskernzähler 3022 umzusetzen. Im Gegensatz zur Einzelpartikelanalyse kann bei diesem Vorgehen allerdings die Partikelkonzentration für zwei oder mehr verschiedene Größenklassen niemals gleichzeitig, sondern immer nur zeitlich nacheinander gemessen werden. Es ist deshalb nicht sinnvoll möglich, die gemessenen Partikelkonzentrationen über mehrere Größenklassen zu integrieren, wie dies beispielsweise im Zuge der vorliegenden Arbeit mit den ursprünglich 128 Klassen des OPC getan wurde, um für die einzelnen (jetzt zusammengefassten 12) Größenklassen eine bessere statistische Absicherung des Konzentrationssignals zu erreichen (vgl. Kapitel 3.2., Aufbau der Messanlage). Ungünstigerweise sind die Größenklassen beim TSI 3071 nun sehr schmal ausgelegt, so dass die Anzahldichten in den einzelnen Klassen jeweils wieder relativ klein (maximal etwa 100 Teilchen pro $\mathrm{cm}^{3}$ Luft) sind. Zieht man weiter in Erwägung, dass der TSI 3022 im Vergleich zum bereits erwähnten TSI 3010 ein drei mal kleineres Luftvolumen pro Zeiteinheit untersucht, wird deutlich, dass sich das Problem der statistischen Sicherheit zwangsläufig sowohl bei der Konzentrationsbestimmung als auch bei der Berechnung der Depositionsgeschwindigkeit für das TSI - System in ähnlicher Weise wie beim eingesetzten OPC stellen muss. Erschwerend kommt noch hinzu, dass das Signal der Partikelkonzentration beim Durchgang durch den Größen-Fraktionierer nicht zuletzt auf Grund der sehr geringen Ansauggeschwindigkeit von 1 1/min und insbesondere auf Grund des deutlich verlängerten Ansaugweges stark verschmiert wird. Aus diesen Gründen ist es nicht überraschend, wenn es sich in der vorliegende Arbeit gezeigt hat, dass vertikale Partikelflüsse zwischen Atmosphäre und Erdoberfläche mit der zur Verfügung stehenden Kombination aus Kondensationskernzähler TSI 3022 und Größen-Fraktionierer TSI 3071 und mit Hilfe der Eddy - Kovarianzmethode nicht sinnvoll bestimmt werden können. 
Neben der statistischen Unsicherheit hat sich vor allem das Auftreten großer relativer Luftfeuchten ( $>95 \%$ ) als Einschränkung für die Anwendung der Eddy - Kovarianzmethode für die Bestimmung des vertikalen Partikelflusses herausgestellt. Unter diesen Bedingungen werden mit der Kovarianzmethode in Hinblick auf den Stoffaustausch zwischen Atmosphäre und Vegetation unrealistisch hohe, positive wie negative Depositionsgeschwindigkeiten ermittelt. Auch dieses Problem ist bereits prinzipiell bekannt. So sagt FAIRALL (1984) signifikant große, aufwärtsgerichtete Scheinflüsse für Messungen über dem Meer voraus. Wie in der vorliegenden Arbeit gezeigt wurde, ist für das Auftreten großer relativer Feuchten jedoch ein etwas differenziertere Betrachtung notwendig, da die Richtung der berechneten Depositionsgeschwindigkeiten für unterschiedliche Partikelgrößen nicht zwangsläufig identisch sein muss. Da es sich aber in Hinblick auf den zu untersuchenden Stoffaustausch zwischen Atmosphäre und Vegetation letztlich immer um Scheinflüsse handelt, müssen diese berechneten Werte jedoch in jedem Fall verworfen werden. Dies bedeutet aber, dass - je nach Jahreszeit und Untersuchungsort - für mehr oder weniger große Zeiträume keine Aussagen zur Partikeldeposition mit Hilfe der Eddy-Kovarianzmethode getroffen werden können. Dies ist signifikant, zumal die mikrometeorologischen Methoden für die Bestimmung der Trockenen Deposition luftgetragener Partikel gerade als eine Möglichkeit angesehen werden, kontinuierliche Messungen über lange Zeiträume zu realisieren. Gleichzeitig ist es so leider auch nicht möglich, die gegenüber Zeiträumen mit einer relativen Luftfeuchte $<95 \%$ stark erhöhte Anzahldichte von Partikeln mit einem Durchmesser $>0,5 \mu \mathrm{m}$ für eine statistisch sichere Berechnungen der Depositionsgeschwindigkeit auszunutzen.

Gallagher ET AL. (1997) umgehen das genannte Problem des Auftretens unrealistisch hoher Scheinflüsse bei großen relativen Luftfeuchten, indem sie einen Analysator verwenden, der die Partikelkonzentration als Funktion des Trockenradius der Teilchen bestimmt. Da es sich hierbei um eine konservative Eigenschaft des atmosphärischen Aerosols handelt, werden ihre Ergebnisse nicht durch Änderungen der relativen Luftfeuchte beeinflusst. Der in der vorliegenden Arbeit verwendete OPC ist leider nicht dafür ausgelegt, den Trockenradius von Partikeln zu bestimmen. Für zukünftige Arbeiten am IBK ist zu überprüfen, ob ein entsprechender Umbau sinnvoll möglich ist. Dabei muss insbesondere empirisch untersucht werden, ob eine eindeutige Zuordnung zwischen Trocken- und Feuchtradius möglich ist und wenn ja, welcher Trockenradius - in Abhängigkeit von der Luftfeuchte - welchem Feuchtradius entspricht. Im Rahmen dieser Arbeit konnte diese umfangreiche technische Arbeit nicht geleistet werden.

\subsection{Partikelkonzentration und Partikelspektrum}

Verschiedene Aspekte des zeitlichen Verlaufs der Partikelkonzentration, insbesondere auch in Hinblick auf die beobachtete Parallelität zum Verlauf der relativen Feuchte, sind bereits im Kapitel 4 andiskutiert worden. Es kann zusammengefasst werden, dass der Konzentrationsverlauf insgesamt wohl von einer Vielzahl von Prozessen des Partikelantransports, der 
Partikelbildung und der Partikelumwandlung sowie der Partikeldeposition geprägt ist, deren konkrete Einzelbeiträge nur im Rahmen einer umfassenden Modellierung bestimmt werden können. Dass sich das Konzentrationsminimum am frühen Nachmittag sowohl im mittleren Tagesgang als auch in den aktuellen Tagesgängen fast aller Messtage wiederfindet, deutet auf einen bedeutenden Beitrag der vertikalen Austauschvorgänge auf den Verlauf der aktuellen Partikelkonzentration in der Atmosphäre hin.

Diese Feststellungen beziehen sich jedoch ausschließlich auf den vom OPC abgedeckten Größenbereich luftgetragener Partikel. Dieser Bereich ist deutlich eingeschränkt. Mit der unteren Zählgrenze von ca. 0,2 $\mu \mathrm{m}$ kann der OPC leider nur noch denjenigen Anteil atmosphärischer Partikel detektieren, der bereits jenseits des Konzentrationsmaximums zwischen 0,01 und 0,1 $\mu \mathrm{m}$ Partikelradius anzusiedeln ist. Mit der gemessenen mittleren Partikelkonzentrationen von 800 Partikel $/ \mathrm{cm}^{3}$ wird also nur ein kleiner Anteil der insgesamt in der Luft vorhandenen Partikel erfasst. Tatsächlich wurden von dem zeitweise parallel betriebenen CPC ohne Größen-Fraktionierer mehr als die doppelte Anzahl an Partikeln gefunden. Für Partikel mit einem Durchmesser $<0,2 \mu \mathrm{m}$ ist bekannt, dass der Tagesgang der Partikelkonzentration - im Gegensatz zu den gefundenen Tagesgängen für den durch den OPC abgedeckten Größenbereich - maßgeblich auch von Partikelbildungsereignissen bestimmt wird (z.B. MÄKELÄ ET AL., 1997).

Die Untersuchungen zur Verschiebung des Konzentrationsmaximums des Partikelspektrums hin zu größeren Durchmessern bei hohen relativen Luftfeuchten haben gezeigt, dass die Trennung zwischen dem System der luftgetragenen Partikel einerseits und dem System der Nebel- und Wassertropfen der Atmosphäre andererseits künstlich ist und für Langzeitmessungen nicht durchgehalten werden kann. Die theoretischen Kurven des Partikelspektrums von JUNGE (1963) sind die Ergebnisse von "Schön-Wetter-Messungen", die für den betrachteten Standort bei hohen relativen Luftfeuchten und/oder tiefliegenden Wolken deutlich zu modifizieren sind. Da solche Randbedingungen auf der gewählten Untersuchungsfläche im Solling häufig vorkommen, ist diese Tatsache zukünftig bei der Betrachtung vertikaler Stofftransporte zu berücksichtigen.

\subsection{Depositionsgeschwindigkeit luftgetragener Partikel über Wald}

\subsubsection{Vergleich der gefundenen Resultate mit Literaturangaben}

Die Ergebnisse der vorliegenden Arbeit reihen sich in eine Gruppe von Untersuchungen ein (z.B. HicKS ET AL., 1982; BRÜCKMANN ET AL., 1989; HiCKS ET AL., 1989; WARAGHAi \& Gravenhorst, 1989; Hertlein, 1990; Constantin, 1993; Gallagher et AL., 1997), die für luftgetragene Partikel deutlich höhere Beträge der Depositionsgeschwindigkeit über Wäldern bestimmen, als dies in theoretischen Arbeiten (SLINN, 1982; GIORGI, 1986; PETERS \& EIDEN, 1992) vorhergesagt bzw. aus Arbeiten mit künstlichen monodispersen Aerosolen (Sehmel \& Hodgson, 1980; JonAs, 1994) abgeleitet wurde. Die Diskrepanz zwischen den 
theoretischen Modellen und den realen Feldmessungen wird dabei im allgemeinen auf die Unvollkommenheit der Modelle, namentlich auf eine Überschätzung der Dicke der viskosen Unterschicht resp. auf eine fehlerhafte Abbildung der Transportprozesse in dieser Schicht zurückgeführt. Die gefundenen Depositionsgeschwindigkeiten sind gleichzeitig - und dies wiederum in Übereinstimmung mit der Reihe der genannten Arbeiten - um fast eine Größenordnung höher als gemessene Depositionsgeschwindigkeiten über Gras- und Wasserflächen (z.B. WeSELY ET AL., 1985; DUAN ET AL., 1988).

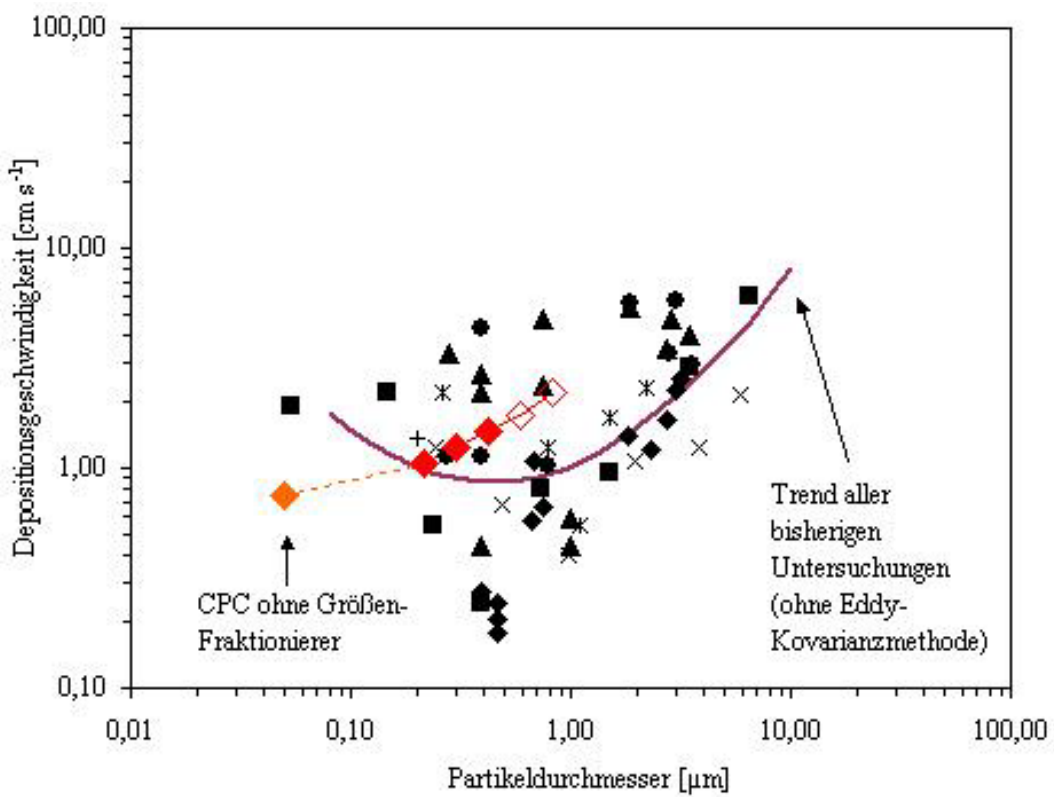

Abb. 43: Vergleich der Ergebnisse der bisherigen Depositionsmessungen auf der F1-Fläche mit den Ergebnisses der vorliegenden Arbeit (rot). MMD: mittlerer Massendurchmesser
- Marques (1998): Abwaschwerfahren (MMD)

- Marques (1998): Elektronenrikroskop

A Ibrom (1993): Kronenraumbilanzienung(MMD)

$\times$ Gravenhorst \& Waraghai (1990): Elektronenrmikroskop

* Höfken et al. (1981): Kronenraum- und Streubilanzierung (MMD)

- Constantin (1993): Nettokronentraufe (MMD)

+ Brückmann et al. (1989): Radionuklidbilanzienung

- Eddy-Kovarianzmethode (eigene Ergebrisse)

Absolut stimmen die Ergebnisse insbesondere mit denen von HERTLEIN (1990) und Gallagher ET AL. (1997) gut überein. Die bestimmten Depositionsgeschwindigkeiten sind aber beispielsweise höher als diejenigen von WYERS \& DUYZER (1997), die die Deposition von Sulfat (MMD ca. 0,6 $\mu \mathrm{m}$ ) mit Hilfe der Gradientmethode bestimmt haben. Die Autoren finden eine mittlere Depositionsgeschwindigkeit von nur $0,7 \mathrm{~cm} / \mathrm{s}$ und sehen diese durch gleichlautende Ergebnisse von HICKS ET AL. (1982) und HICKS ET AL. (1989) (beides Kovarianzmethode) bestätigt. Für den betrachteten Fichtenaltbestand sind die gefundenen Resultate im Vergleich zu bisherigen Untersuchungen auf der F1-Fläche weder ausgesprochen niedrig noch besonders hoch, sondern entsprechen in etwa dem arithmetischen Mittel aller bisherigen Messungen. Die Ergebnisse sind so etwas niedriger als beispielsweise 
die meisten von IBROM (1993) angegebenen Depositionsgeschwindigkeiten, gleichzeitig aber deutlich höher als die von MARQUES (1998) mit dem Abwaschverfahren bestimmten Beträge. Obwohl alle Messungen am gleichen Standort stattfanden, sind die Ergebnisse aber untereinander nur eingeschränkt vergleichbar, da die einzelnen Untersuchungen mit unterschiedlichen Methoden, bezogen auf unterschiedlich definierte Partikeldurchmesser und $\mathrm{zu}$ unterschiedlichen Zeiten - und damit unter unterschiedlichen meteorologischen Bedingungen - durchgeführt wurden (Abb. 43).

Das vermutlich interessanteste und wichtigste Ergebnis der vorliegenden Arbeit ist zweifelsohne die Beobachtung, dass die Depositionsgeschwindigkeiten im Größenbereich zwischen 0,2 und 0,5 $\mu \mathrm{m}$ Durchmesser mit der Partikelgröße ansteigen. Dies stimmt mit den Ergebnissen der größenspezifischen Messungen von GALlaGHER ET AL. (1997) sowie NeUmann \& HARTOG (1985) (beide Kovarianzmethode, letztere Messung über Gras) gut überein. Gleichzeitig steht diese Aussage zunächst aber im Kontrast beispielsweise zu den Resultaten von CONSTANTIN (1993), der mit einem halbempirischen Modell das absolute Minimum der Depositionsgeschwindigkeit für die gleiche untersuchte Fläche (F1-Fläche) bei einem Durchmesser von etwa $2 \mu \mathrm{m}$ modelliert (Abb. 44). Andere Autoren sehen das absolute Minimum der Depositionsgeschwindigkeit bei etwa $1,5 \mu \mathrm{m}$ Durchmesser (HÖFKEN \& GRAVENHORST, 1983) bzw. bei noch kleineren Partikeldurchmesser von 0,5 bis $1 \mu \mathrm{m}$, wie beispielsweise Wiman \& Agren (1985), PEters \& EIdEn (1992) (beide Modellrechnungen), GRAVENHORST \& WARAGHAI (1990) (Elektronenmikroskop) und MARQUES (1998) (Abwaschverfahren).

Abb. 44: Vergleich der berechneten Depositionsgeschwindigkeit (rot, eigene Ergebnisse) mit modellierten Werten von CONSTANTIN (1993)

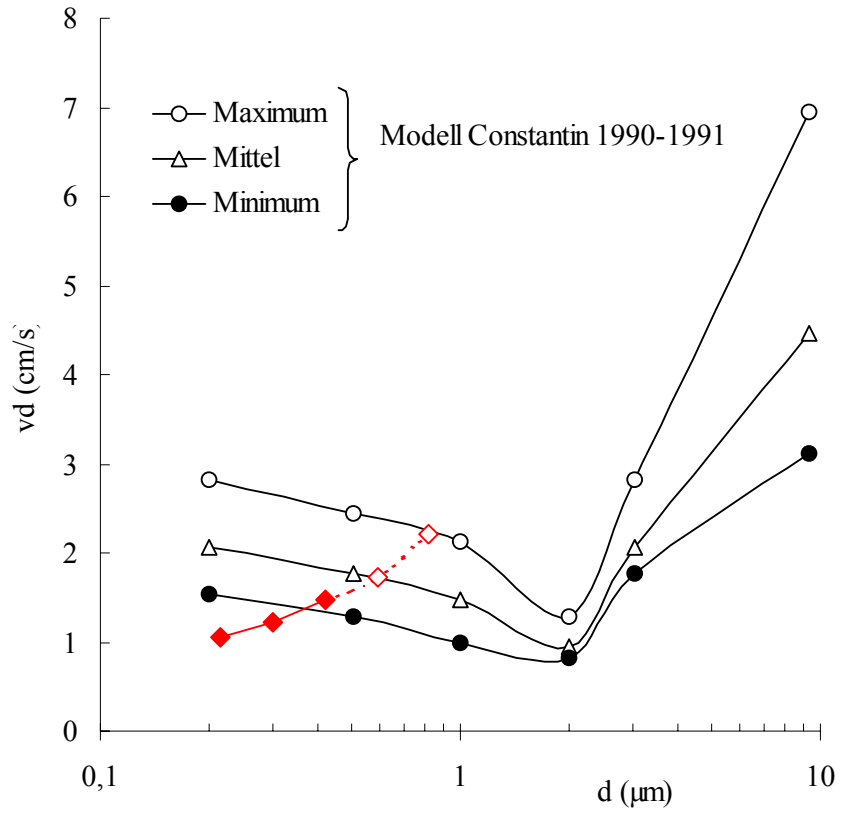

Die beiden scheinbar grundverschiedenen Ansichten über den Verlauf der Depositionsgeschwindigkeit im Bereich der Partikel von 0,2 bis $0,4 \mu \mathrm{m}$ Durchmesser lassen sich 
zusammenführen, wenn man das von CONSTANTIN (1993) und den anderen genannten Autoren bestimmte Minimum bei 1 oder $2 \mu \mathrm{m}$ nicht als absolutes, sondern lediglich als ein lokales Minimum versteht, welches neben einem ebenfalls lokalen Maximum der Depositionsgeschwindigkeit bei etwa 0,5 $\mu \mathrm{m}$ Durchmesser existiert. Diese Ansicht wird von GALlagher ET AL. (1997) vertreten, die das absolute Minimum der Depositionsgeschwindigkeiten luftgetragener Partikel dann bei einem Durchmesser von $0,1 \mu \mathrm{m}$ vermuten. Da unterhalb dieser Größe jedoch keine Messungen vorliegen, kann über das Zutreffen dieser Aussage nur spekuliert werden.

Im Gegensatz zu anderen Eddy - Kovarianzmessungen wurden in der vorliegenden Arbeit keine Partikelflüsse aus dem Fichtenbestand heraus in die Atmosphäre hinein beobachtet. Ausnahmen sind Einzelwerte, die als Ausreißer interpretiert und verworfen werden können und deren Auftreten sich mit der Partikelgröße und damit der statistischen Unsicherheit der Einzelwerte häufen, sowie aufwärtsgerichtete Scheinflüsse in Zeiten hoher relativer Feuchte, die aus den in Kapitel 4.1.5 dargestellten Gründen ebenfalls ausselektiert wurden. Das Nichtauftreten aufwärtsgerichteter Partikelflüsse lässt sich wahrscheinlich mit der Größe der betrachteten Partikel (Durchmesser 0,2 $\mu \mathrm{m}$ und größer) erklären. So finden GALLAGHER ET AL. (1997) zeitweise aufwärtsgerichtete Flüsse über einen Douglasienforst nur für Partikel $<0,18 \mu \mathrm{m}$. In den Größenklassen $>0,18 \mu \mathrm{m}$ Durchmesser sind die Partikelflüsse dagegen für die gesamte Beobachtungszeit ausschließlich zur Erdoberfläche hin gerichtet. Während Wälder für Partikel $<2 \mu \mathrm{m}$ Durchmesser zeitweise also durchaus als Quelle fungieren können, ist dies für größere Partikel netto scheinbar nicht gegeben.

\subsubsection{Abhängigkeit der Depositionsgeschwindigkeit von der atmosphärischen Turbulenz}

Es ist eine allgemein akzeptierte Auffassung, dass der Betrag der Depositionsgeschwindigkeit nicht nur vom Partikeldurchmesser, sondern auch von der Ausprägung der Turbulenz in der Planetarischen Grenzschicht abhängt. Viele Autoren geben dabei einen von der Stabilität der Atmosphäre unabhängigen linearen oder exponentiellen Zusammenhang zwischen der Depositionsgeschwindigkeit und der Schubspannungsgeschwindigkeit $u *$ an (z.B. VERMEULEN ET AL., 1997). Bei genauerer Betrachtung zeigt es sich jedoch, dass hier oft aus der Not eine Tugend gemacht wird. Entweder sind die Einzelmessungen mit solch hohen statistischen Fehlern versehen sind, dass man ohnehin nicht umhin kommt, die Resultate über längere Zeiträume, unter Umständen sogar über eine ganze Messkampagne zu mitteln (z.B. WYERS \& DUYZER, 1997), oder aber die verwendeten Messmethoden lassen von vornherein keine Auflösung des zeitlichen Verlaufs der Depositionsgeschwindigkeiten in der Größenordnung von einer Stunde oder weniger zu (z.B. WARAGHAI \& GRAVENHORST, 1989). In beiden Fällen ist es selbstredend schlecht möglich, differenziertere Angaben zur Abhängigkeit der Depositionsgeschwindigkeit von der Stabilität der Atmosphäre zu treffen. 
Im Rahmen der vorliegenden Arbeit wurden Eddy - Kovarianzmessungen über einen Zeitraum von zwei Jahren durchgeführt, so dass - trotz der hohen statistischen Unsicherheit der Einzelwerte - auch der Einfluss der Stabilität der Atmosphäre auf die Depositionsgeschwindigkeit luftgetragener Partikel herausgearbeitet werden konnte. Erwartungsgemäß zeigt sich zunächst, dass die Depositionsgeschwindigkeiten für alle Größenklassen mit $u *$ positiv korreliert sind. Hierin unterschieden sich die erzielten Ergebnisse nicht von den oben erwähnten Arbeiten. Die grundsätzliche Abhängigkeit von $u *$ ist nicht überraschend, weil die Wirkungsstärke vieler Prozesse, die an der Trockenen Deposition luftgetragener Partikel beteiligt sind (insbesondere Impaktion und turbulente Diffusion, vgl. Kapitel 1.3.2.1), mit der Intensität der horizontalen Windgeschwindigkeit bzw. der Schubspannungsgeschwindigkeit zunimmt. Dieser Regelungsmechanismus wird aber offensichtlich noch einmal überlagert durch den Einfluss thermisch induzierter Effekte: bei stabiler Schichtung wird der vertikale Partikelantransport aus der Atmosphäre in den Pflanzenbestand hinein signifikant abgebremst, während er bei labiler Schichtung umgekehrt intensiviert wird. Insofern reicht es für Betrachtungen der Partikeldeposition über Zeiträume $<24$ Stunden nicht aus, die Depositionsgeschwindigkeit luftgetragener Partikel ausschließlich über $u *$ oder ein anderes Maß der mechanisch induzierten Turbulenz zu parametrisieren. Weiter kann vermutet werden, dass der klare Tagesgang der Depositionsgeschwindigkeit luftgetragener Partikel (vgl. Abb. 38) wenigstens teilweise auch auf thermische Effekte zurückzuführen ist. So zeigt der Tagesgang des fühlbaren Wärmeflusses eine starke Ähnlichkeit mit dem der Depositionsgeschwindigkeit, was gleichermaßen allerdings auch für den Tagesgang der Schubspannungsgeschwindigkeit festgestellt werden kann. Der mittlere Gang der Windgeschwindigkeit ist dagegen durch ein deutliches Minimum am frühen Abend gekennzeichnet, das sich in dieser Form im Tagesgang der Depositionsgeschwindigkeit nicht wiederfindet.

$\mathrm{Da}$ in der vorliegenden Arbeit vornehmlich Partikel mit einem Durchmesser $<0,5 \mu \mathrm{m}$ untersucht wurden, ist der gefundene statistische Zusammenhang zwischen der Depositionsgeschwindigkeit und der Schubspannungsgeschwindigkeit kleiner als bei Arbeiten mit größeren Partikel. So bestimmen z.B. Vermeulen ET AL. (1997) bei Eddy - Kovarianzmessungen mit Nebeltropfen einen linearen Korrelationskoeffizienten von 0,83. Dieses Ergebnis steht aber nicht in Widerspruch $\mathrm{zu}$ den eigenen Resultaten, sondern unterstützt vielmehr die Auffassung von GRAVENHORST \& WARAGHAI (1990), die für die Stärke des Zusammenhangs zwischen Depositionsgeschwindigkeit und Windgeschwindigkeit selbst eine Abhängigkeit von der Partikelgröße vorhersagen.

\subsubsection{Fehlerabschätzung}

Die Resultate der vorliegenden Arbeit stimmen in der Grundaussage mit den Resultaten ähnlicher experimenteller Untersuchungen überein, lassen aber im Detail auch Differenzen erkennen. Für einen Vergleich mit Untersuchungen, die zum Teil auf anderen Methoden als der Eddy - Kovarianzmethode beruhen, ist es deshalb sinnvoll, die Korrektheit der eigenen Resultate zu überprüfen. Die hohen statistischen Unsicherheiten der Einzelbeobachtungen, die 
ein klare, methodisch bedingte Schwäche der vorliegenden Arbeit darstellen, wurden bereits diskutiert. Da es sich hierbei um zufällige Fehler handelt, werden die berechneten Depositionsgeschwindigkeiten damit prinzipiell aber weder unter- noch überschätzt. Im folgenden sollen vornehmlich die systematische Fehler bei der Bestimmung der Deposition betrachtet werden.

Die grundlegende Annahme aller meteorologischen Methoden, dass der Betrag des Vertikalflusses eines atmosphärischen Spurenstoffes (hier des vertikalen Partikelflusses) in bzw. aus der Atmosphäre dem Stoffeintrag bzw. Stoffaustrag eines betrachteten Bestandes äquivalent ist, kann für den betrachteten Untersuchungsstandort im allgemeinen als gegeben angesehen werden. Dies haben die zahlreichen Eddy - Kovarianzuntersuchungen auf der F1-Fläche (z.B. IBROM, 1999) gezeigt. Auf Grund der exponierten Lage des Hochsollings wird jedoch für die Nachtstunden ein horizontaler Kaltluftabfluss vermutet. Dies könnte zur Folge haben, dass die nächtliche Partikeldeposition überschätzt wird, da die über der F1-Fläche vertikal abwärts transportierten Partikel möglicherweise in einem größeren Gebiet deponiert werden.

Abgesehen von diesem grundsätzlichen - auf die Nachtstunden beschränkten - Problem, reduziert sich die Fragestellung nach der Korrektheit der bestimmten Depositionsgeschwindigkeiten so auf die Frage nach der Korrektheit der Bestimmung des vertikalen Flusses luftgetragener Partikel. Dieser ergibt sich nach FAIRALL (1984) vollständig aus:

$$
\begin{aligned}
F= & \overline{w^{\prime} c^{\prime}}-v_{g} \bar{c}-\overline{v_{g}^{\prime} c^{\prime}}-\overline{w_{s}^{\prime} c^{\prime}}-D_{P} \partial c / \partial z \\
\text { mit: } v_{g} & \text { Sedimentationsgeschwindigkeit } \\
w_{s} & \text { Vertikalkomponente der slip Geschwindigkeit } \\
D_{P} & \text { molekularer Diffusionskoeffizient }
\end{aligned}
$$

Der erste Term $\overline{w^{\prime} c^{\prime}}$ beschreibt den turbulenten Partikeltransport. Nur dieser wird durch die Eddy - Kovarianzmethode bestimmt. Der zweite Term $v_{g} \bar{c}$ repräsentiert den Partikelfluss in Folge der Schwerkraft der Erde. Dieser ist für die betrachteten Partikel vernachlässigbar klein (vgl. Abb. 9). Für Partikel, für die nicht mehr vernachlässigt werden kann, ist $v_{g}$ leicht zu berechnen, und der mit der Eddy - Kovarianzmethode bestimmte Fluss kann entsprechend korrigiert werden. Der dritte Term $\overline{v_{g}^{\prime} c^{\prime}}$ berücksichtigt Veränderungen der Sedimentationsgeschwindigkeit in Folge von Größenänderungen der Partikel bei Fluktuationen der relativen Feuchte. Wie bereits mehrfach erwähnt wurde, findet ein signifikantes Partikelwachstum durch die Anlagerung von Wassermolekülen ausschließlich bei sehr hohen relativen Feuchten $>95 \%$ statt. Da die berechneten Depositionsgeschwindigkeiten für diese Feuchten in der vorliegenden Arbeit nicht berücksichtigt wurden, kann auch dieser Term ignoriert werden. Der vierte und der fünfte Term, $\overline{w_{s}^{\prime} c^{\prime}}$ und $D_{P} \partial c / \partial z$ beschreiben Prozesse (molekulare Diffusion, Thermophorese), die vor allem in der molekular-viskosen Unterschicht von Bedeutung sind. In einer Messhöhe von $39 \mathrm{~m}$ haben beide jedoch vernachlässigbar kleine Anteile am Gesamtfluss $F$ (FAIRALL, 1984).

Es kann deshalb festgehalten werden, dass der Partikelfluss in der Messhöhe von $39 \mathrm{~m}$ mit dem Kovarianzprodukt $\overline{w^{\prime} c^{\prime}}$ ausreichend genau beschrieben werden kann. Wie bisherige 
Messungen auf der Untersuchungsfläche gezeigt haben, befindet sich das Messsystem in der gewählten Messhöhe im Bereich der Prandtl-Schicht (LAUBACH, 1991; LöFFLER, 1994). Der in dieser Höhe bestimmte Partikelfluss repräsentiert also - bei Abwesenheit von horizontalen Flussdivergenzen sowie bei Abwesenheit von Quellen und Senken zwischen der Vegetation und der Atmosphäre - auch den Stoffeintrag in den betrachteten Bestand hinreichend genau. Diese Höhenunabhängigkeit des Partikelflusses lässt sich aber nicht automatisch auch auf die Depositionsgeschwindigkeit $v_{d}$ übertragen. Weil die Partikelkonzentration mit der Höhe variiert, muss dies auch für $v_{d}$ gelten. Im allgemeinen ist dabei zu erwarten, dass die Partikelkonzentrationen in niedrigeren Höhen, wie beispielsweise direkt im Bestand, kleiner sind als in der Messhöhe von $39 \mathrm{~m}$. Folglich werden die Beträge von $v_{d}$, die mit der Kovarianzmethode bestimmt werden, tendenziell etwas höher sein als Ergebnisse von Untersuchungen, die innerhalb eines Bestandes beispielsweise mit Hilfe direkter Verfahren durchgeführt werden, und deren Ergebnisse sich auch auf die dort bestimmten Partikelkonzentrationen beziehen. In der sehr labilen Prandtl-Schicht über der rauhen Oberfläche eines Waldes sind die vertikalen Konzentrationsdifferenzen allerdings generell sehr klein (BUZORIUS ET AL., 1998; Hertlein, 1990). Für Partikel mit einem Durchmesser von 0,5 $\mu \mathrm{m}$ beträgt die Konzentrationsdifferenz für den betrachteten Fichtenbestand nach CONSTANTIN (1993) zwischen $39 \mathrm{~m}$ und $14 \mathrm{~m}$ Höhe über Erdboden weniger als $5 \%$. Die resultierende Veränderung der Depositionsgeschwindigkeit als Funktion der Messhöhe ist also gering.

Bei Eddy - Kovarianzuntersuchungen besteht grundsätzlich die Möglichkeit, dass der turbulente Fluss $\overline{w^{\prime} c^{\prime}}$ unterschätzt wird, da unter Umständen nicht wirklich alle zum Fluss beitragenden Fluktuationen der Partikelkonzentration $c$ erfasst werden, oder die Beträge der Fluktuationen der vertikalen Windgeschwindigkeit $w$ zu klein bestimmt werden. Deshalb werden entsprechende Korrekturen angewendet (vgl. Abschnitt 2.2.4), die selbst wiederum prinzipiell auch potentielle Ursachen für eine Fehlabschätzung des Vertikalflusses sind. So zweifeln FOKEN \& WICHURA (1996) beispielsweise die Gültigkeit der Übertragung einer im Windkanal erstellten Korrekturmatrix für ein Ultraschallanemometer grundsätzlich an. Zwar erkennen sie an, dass $w$ ohne eine Korrektur in Hinblick auf die Beeinflussung des Windvektors durch das Ultraschallanemometer signifikant $\mathrm{zu}$ niedrig bestimmt wird, postulieren aber gleichzeitig, dass die Anwendung einer unter den laminaren Strömungsverhältnissen eines Windkanals erstellten Korrektur für reale Feldmessungen eine starke Überschätzung des Flusses zur Folge hätte. Diese Einschätzung ist bis dato keinesfalls bewiesen, aber auch nicht schlüssig widerlegt. Ein Indiz für die prinzipielle Richtigkeit der Korrektur von $w$ aus Windkanaldaten gibt IBROM (1999), der mit einer solchen Korrektur eine deutlich bessere Schließung der Energiebilanz erzielt als ohne Korrektur. Auch CHRISTEN ET AL. (2001) schließen aus einer Feldstudie mit 19 verschiedenen Ultraschallanemometern, dass für das Metek-Sonic USA1 bei der Anwendung einer in einem Windkanal erstellten Korrektur auf die Messdaten in den meisten Fällen eine substantielle Verbesserung der Übereinstimmung mit einem Referenzgerät erreicht werden kann. Deshalb wurde auch für diese Arbeit entschieden, die im Windkanal der ETH Zürich erstellte Korrekturmatrix (siehe Anhang) auf den aufgenommenen Daten anzuwenden. Den Fall angenommen, dass die 
Bedenken von FOKEN \& WICHURA (1996) berechtigt sind, würde dies eine Überschätzung aller ermittelten Depositionsgeschwindigkeiten um maximal $25 \%$ bedeuten.

Zusammengefasst muss festgehalten werden, dass für die in der vorliegenden Arbeit durchgeführte experimentelle Bestimmung der Depositionsgeschwindigkeit luftgetragener Partikel mit Hilfe der Eddy - Kovarianzmethode eine Reihe potentieller Fehlermöglichkeiten existieren. Dabei kann nicht abschließend geklärt werden, ob die tatsächlichen Depositionsgeschwindigkeiten durch die berechneten Beträge tendenziell eher über- oder unterschätzt werden. In jedem Fall ist es wahrscheinlich, dass potentielle Fehler sich auf die Berechnung der Depositionsgeschwindigkeit in den unterschiedlichen Größenklassen in gleicher Art und Weise auswirken würden. Dies bedeutet, dass die Aussagen zum relativen Verhältnis der Depositionsgeschwindigkeiten der einzelnen Größenklassen untereinander als in jedem Fall zutreffend angesehen werden können. Die gute Übereinstimmung mit den Werten aus der Literatur deutet darüber hinaus darauf hin, dass die berechneten Werte auch absolut sehr gut mit der Realität übereinstimmen.

Bei der bisherigen Betrachtung unberücksichtigt geblieben ist die Tatsache, dass die Größenbestimmung der luftgetragener Partikel bei dem verwendeten optischen Partikelzähler nur indirekt erfolgt und auf Vergleichsmessungen mit künstlichen Partikeln, deren optischen Eigenschaften nicht mit denjenigen atmosphärischer Partikel übereinstimmen, beruht. Hier sind also systematische Fehler möglich. Es ist jedoch festzustellen, dass die Durchmesserbestimmung bei anderen Verfahren häufig noch mit viel größeren Unsicherheiten belastet ist. So werden die Partikel bei Untersuchungen von Blattoberflächen mit dem Elektronenmikroskop im trockenen Zustand betrachtet, so dass die Partikelgröße wahrscheinlich im Vergleich zu der ursprünglichen Größe der luftgetragenen Partikel im feuchten Zustand systematisch zu klein bestimmt wird. In jedem Fall hat die Unterschiedlichkeit der Verfahren der Größenbestimmung zur Folge, dass Vergleiche zwischen verschiedenen Untersuchungen generell als problematisch anzusehen sind. Für eine detaillierte Charakterisierung dieses Problems sind umfangreiche Vergleichsuntersuchungen notwendig.

\subsubsection{Ausblick}

Die zukünftige Entwicklung unserer Wälder und Forste wird in starkem Maße durch Art und Umfang des atmosphärischen Stoffeintrages in diese Systeme geprägt werden. Deshalb ist es sinnvoll und notwendig, diesen Stoffeintrag im Zuge eines Depositionsmonitorings möglichst flächendeckend und kontinuierlich zu überwachen, um negative Entwicklungen frühzeitig erkennen und beeinflussen zu können. Die Methoden eines solchen Depositionsmonitorings müssen preiswert und routinemäßig einsetzbar sein. Die beste Methodik nutzt nichts, wenn nur eine kleine Anzahl spezialisierter Forschungsinstitute mit ihr qualitativ ausreichende Ergebnisse erzielen kann (SPRANGER, 1994). 
Die vorliegende Arbeit zeigt, dass die Eddy - Kovarianzmethode für sich genommen diese Anforderung nicht erfüllen kann. Die erfolgreiche Anwendung der Methode für die Bestimmung des vertikalen Partikeltransports setzt nicht nur erhebliches Spezialwissen und technisches Know-how voraus, sondern insbesondere auch die Verfügbarkeit leistungsfähiger und deshalb wahrscheinlich kostspieliger Analysatoren, die zudem sogar erst noch entwickelt werden müssen. Auch sind mit der Eddy - Kovarianzmethode allein keine Aussagen zur Art der deponierten Stoffe möglich. Die mit der Methode bestimmten Depositionsgeschwindigkeiten sind folglich in erster Line nur "Hilfsgrößen", die im Rahmen anderer, häufig halbempirischer Methoden, wie der sogenannten Konzentrationsmethode, als wichtige Eingangsgrößen für die quantitative und qualitative Bestimmung des atmosphärischen Stoffeintrags in Wälder und andere Ökosysteme fungieren.

Zukünftige mikrometeorologische Arbeiten auf dem Gebiet der Partikeldeposition werden vor allem die Problematik der Geräteentwicklung vorrangig beachten müssen, um die bereits bekannten Probleme bei der Anwendung der Kovarianzmethode für luftgetragene Partikel lösen oder umgehen zu können. Kann diese umfangreiche Aufgabe beispielsweise im Rahmen einer interdisziplinärer Zusammenarbeit erfolgreich gelöst werden, wird die Eddy - Kovarianzmethode eine hervorragende Möglichkeit darstellen, vorhandene Modelle für die Bestimmung der Partikeldeposition zu validieren. Gerade im Hinblick auf die zahlreichen systematischen Unzulänglichkeiten anderer empirischer Methoden, wie der Bestimmung der Partikeldeposition mit Hilfe der Nettokronentraufe, des Abwaschverfahrens oder anderer direkter Verfahren, wird der Eddy - Kovarianzmethode hier in Zukunft eine bedeutende Rolle spielen. 


\section{Zusammenfassung}

Ziel der vorliegenden Arbeit war die Bestimmung der Depositionsgeschwindigkeit luftgetragener Partikel über einem Fichtenaltbestand im Solling. Als Untersuchungsmethode wurde die Eddy-Kovarianzmethode ausgewählt, als Messgerät für die zeitlich hochaufgelöste und größenspezifische Bestimmung der Partikelkonzentration stand ein kommerzieller Partikelzähler PCS-2000 der Firma Palas (hiernach: OPC) zur Verfügung.

Für den durch den OPC abgedeckten Größenbereich von 0,2 bis $10 \mu \mathrm{m}$ Durchmesser wurde für den gewählten Untersuchungsstandort eine mittlere Konzentration von knapp 800 Partikel pro $\mathrm{cm}^{3}$ bestimmt. Der Gang der Partikelkonzentration im Tagesverlauf zeigt eine starke Abhängigkeit vom Gang der relativen Feuchte und ist wahrscheinlich von einer Vielzahl von Prozessen des Partikelantransports, der Partikelbildung, der Partikelumwandlung und insbesondere auch der Partikeldeposition bestimmt, deren konkreten Einzelbeiträge nur im Rahmen einer umfassenden Modellierung bestimmt werden können. Das Partikelspektrum ist bei hohen relativen Luftfeuchten durch eine Verschiebung des Konzentrationsmaximums von Partikeln $<0,2 \mu \mathrm{m}$ Durchmesser hin zu Partikel von 1 bis $2 \mu \mathrm{m}$ Durchmesser geprägt. Bei den unter den Bedingungen der hoher Luftfeuchte verstärkt auftretenden Partikeln handelt es sich um Nebeltröpfchen. Nebelbildung und Nebelperioden können mit Hilfe des OPC hervorragend registriert, beobachtet und analysiert werden.

Die mit dem OPC aufgenommenen, zeitlich hochaufgelösten Zeitreihen der Partikelkonzentration sind ausgesprochen stark verrauscht. Dieses Phänomen kann vollständig auf das verwendete Messprinzip der Einzelpartikelanalyse zurückgeführt werden. Als direkte Folge der hohen statistischen Unsicherheit schneller Konzentrationsmessungen mit Einzelpartikelanalysatoren sind auch die aus dem Konzentrationssignal berechneten Depositionsgeschwindigkeiten mit einem großen statistischen Fehler behaftet. Dieser Fehler kann aus der tatsächlichen oder erwarteten Standardabweichung der vertikalen Windgeschwindigkeit, der mittleren Partikelkonzentration, der Größe des auf das Vorhandensein von Partikeln untersuchten Luftvolumens und der Messfrequenz vorhergesagt werden. Je niedriger die Partikelkonzentration und das pro Zeiteinheit untersuchte Luftvolumen ist, umso größer ist die statistische Unsicherheit bei der Flussbestimmung.

Bei einer hohen relativen Luftfeuchtigkeit werden mit der Eddy - Kovarianzmethode Partikelflüsse berechnet, die in Hinblick auf den Stoffaustausch zwischen Vegetation und Atmosphäre nicht logisch erklärt werden können. Diese "Scheinflüsse" sind das Ergebnis einer raschen und stetigen Größenänderung der in der Luft vorhandenen Partikel.

Die sinnvolle Auswertung des aufgenommenen Datensatzes setzt eine umfangreiche Selektion und eine leichte Modifikation der Daten voraus. Die über den gesamten Messzeitraum gemittelten Depositionsgeschwindigkeiten betragen $1,05 \mathrm{~cm} / \mathrm{s}(0,22 \mu \mathrm{m}), 1,23 \mathrm{~cm} / \mathrm{s}(0,30 \mu \mathrm{m})$ 
bzw. 1,47 cm/s $(0,42 \mu \mathrm{m}$ Partikeldurchmesser). Der Betrag der Depositionsgeschwindigkeit steigt somit im Größenbereich von 0,2 bis $0,5 \mu \mathrm{m}$ Partikeldurchmesser mit der Partikelgröße an. Für Partikel mit einem Durchmesser größer 0,5 $\mu \mathrm{m}$ konnte die Depositionsgeschwindigkeit mit Hilfe der Eddy - Kovarianzmethode nicht sinnvoll bestimmt werden. Die Höhe der Depositionsgeschwindigkeit wird maßgeblich von der Stärke der mechanisch induzierten Turbulenz und der Stabilität der Atmosphäre determiniert.

Vorrangige Aufgabe zukünftiger mikrometeorologischer Arbeiten zur Partikeldeposition sollte die Entwicklung geeigneter Partikelmesser für schnelle, größenspezifische Konzentrationsmessungen sein. 


\section{Literatur}

Ahonen, T.; Aalto, P.; RanNik, Ü.; Kulmala, M.; Nilsson, E.D.; Palmroth, S.; YLITALO, H.; HARI, P. (1997): Variations and vertical profiles of trace gas and aerosol concentrations and $\mathrm{CO}_{2}$ exchange in eastern Lapland. Atmospheric Environment, 31, 20, S. 3351-3362.

AitKen, J. (1923): Collected scientific papers (Hrsg.: C. Knott). London, New York: Cambridge University Press.

ARYA, S.P.S. (1988): Introduction to Micrometeorology. San Diego: Academic Press.

BAKER, J.M. (2000): Conditional sampling revisited. Agricultural and Forest Meteorology, 104, S. 59-65.

BALdocchI, D. D.; HiCKS, B. B.; MEYERS, T. P. (1988): Measuring biosphere-atmosphere exchanges of biologically related gases with micrometeorological methods. Ecology, 69, 5, S. 1331-1340.

Beese, F.; Deutschmann, G.; Meiwes, K.J. (1999): Exkursion G5: Solling. Belastungen von Waldböden und deren Sanierung am Beispiel des Sollings. In: Beyme, B.; Böttcher, J.: Nachhaltige Bodennutzung im dritten Jahrtausend. Jahrestagung der Deutschen Bodenkundlichen Gesellschaft 1999 Hannover: Exkursionsführer, Institut für Bodenkunde der Universität Hannover, Mitteilungen der Deutschen Bodenkundlichen Gesellschaft, Bd. 90, S. 307-326.

BERGKVIST, B. (1987): Soil solution chemistry and metal budgets of spruce forest ecosystems in south Sweden. Water Air Soil Pollut., 33, 131-154.

Beswick, K.M.; Gallagher, M.W.; Hummelshou, P.; Pilegaard, K.; Jensen, N.O.; DUYZER, J. (1994): Aerosol exchange to Speulder Forest. In: Borell, P.M.; Borell, P.; Cvitas, T.; Seiler, W.: Transport and transformation of pollutants in the troposphere, The Hague: SPB Academic Publishing, S. 683-688.

Beverland, I. J.; Milne, R.; Biossard, C.; ONeill, D.H.; MoncriefF, J.B.; HewitT, C.N. (1996): Measurement of carbon dioxide and hydrocarbon fluxes from a sitka spruce forest using micrometeorological techniques. Journal of geophysical Research, 101, S. $22,807-22,815$. 
BlANK, S. (2000): Eddy-Korrelationsmessungen von Wasserdampfflüssen über einem Fichtenbestand. Diplomarbeit, Universität Göttingen.

Braud, J.; Noilhan, P.; Bessemoulin, P.; Mascart, P.; HAVERKamP, R.; VAuclin, M. (1993): Bare groud surface heat and water exchanges under dry conditions: observation and parametrization. Boundary-Layer Meteorology, 66, S. 173-200.

BROOK, R.R. (1978): The influence of water vapor fluctuations on turbulent fluxes. Boundary-Layer Meteorology, 15, S. 481-487.

BRÜCKMANN, A.; BRUMME, R.; GRAVENHORST, G. (1989): Radionuklidbilanz von 4 Waldökosystemen nach dem Reaktorunfall in Tschernobyl und eine Bestimmung der trockenen Deposition. Universität Göttingen, Berichte des Forschungszentrums Waldökosysteme, Reihe B, Bd. 9.

Businger, J. A.; ONCLEY, S.P. (1990): Flux measurement with conditional sampling. Journal of atmospheric and oceanic technology, 7, S. 349-352.

ButTerweCK, G. (1991): Natürliche Radionuklide als Tracer zur Messung des turbulenten Austausches und der trockenen Deposition in der Umwelt. Dissertation, Universität Göttingen.

Buzorius, G.; RANNIK, Ü.; MäKelä, J. M.; Vesala, T.; Kulmala, M. (1998): Vertical aerosol particle fluxes measured by eddy covariance technique using conditional particle counter. Journal Aerosol Sci., 29, 1/2, S. 157-171.

Cellier, P.; Brunet, Y. (1992): Flux-gradient relationships above tall plant canopies. Agricultural and Forest Meteorology, 58, S. 93-117.

CERCACOV, V. (1985): Investigation of the atmospheric particulates deposited on leaves using instrumental neutron activation analysis. Atmospheric Environment, 19, S. 681-683.

Chamberlain, A.C. (1960): Aspects of the deposition of radioactive or other gases and particles. Int. J. Air Pollution, 3, S. 63-88.

Charlson, R.J.; SCHWARZ, S.E.; HALeS, J.M.; CeSS, R.D.; COAKLEY, D.A.; HANSEN, J.E.; HofmAnN, D.J. (1992): Climate forcing by anthropogenic aerosols. Science, 255, S. 423-430.

Christen, A.; Gorsel, E. VAn; Vogt, R.; AndrettA, M.; Rotach, M.W. (2001): Ultrasonic anemometer instrumentation at steep slopes: wind tunnel study - field intercomparison - measurements. In: MAP Newsletter, Bd. 15, S. 164-167. 
CONSTANTIN, J. (1993): Stoffeinträge in ein Fichtenwaldökosystem durch Deposition luftgetragener Partikel und Nebeltröpfchen. Universität Göttingen, Berichte des Forschungszentrums Waldökosysteme, Reihe A, Bd. 106.

DWD (1987): Allgemeine Meteorologie. Offenbach: Selbstverlag des Deutschen Wetterdienstes.

Delany, A.; Delany, A. C.; PARKIN, D. W.; GRIfFin, J. J.; GoldBerG, E. D.; ReimanN, B. E. F. (1967): Airborne dust collected at Barbados. Geochim. Cosmochim. Acta, 31, S. 885 909.

DENMEAD, O.T.; RAUPACH, M.R. (1993): Methods for measuring atmospheric gas transport in agricultural and forest systems. In: Agricultural ecosystem effects on trace gases and global climate change, Madison: American Society of Agronomy, ASA special publication, no. 55.

DuAN, B.; FAIRALL, C.W.; THOMSON, D.W. (1988): Eddy correlation measurements of the dry deposition of particles in wintertime. Journal of applied Meteorology, 27, S. 642-652.

DÄMmgen, U.; GRÜNHAGe, L.; HARTwig-HANitZ, B.; JÄGER, H.-J. (1990): Auswirkungen luftgetragener Stoffe auf Vegetation und Boden von Grünlandökosystemen. Landbauforsch. Völkenrode, Bd. 39 (4).

EllenberG, H.; MAYeR, R.; SChaUeRmanN, J. (Hg.) (1986): Ökosystemforschung Ergebnisse des Sollingprojekts: 1966-1986. Stuttgart: Ulmer.

ERISMAN, J. W.; DrAAiJers, G.; DUYZER, J. H.; HOFSChreUder, P.; VAN LEEUWEN, N.; RÖMER, F.; RuiJgroK, W.; Wyers, G. P.; GALlAGHER, M. W. (1997a): Particle deposition to forests - summary of results and application. Atmospheric Environment, 31, 3, S. 321-332.

ERisman, J. W.; DraAiJers, G.; DuYZer, J. H.; HofSChreuder, P.; VAN LEeUWEN, N.; RÖMER, F.; RUIJGROK, W.; WYERS, G. P. (1997b): The aerosol project: introduction and some background information. Atmospheric Environment, 31, 3, S. 315-319.

Eugster, W. (1994): Mikrometeorologische Bestimmung des $\mathrm{NO}_{2}$-Flusses an der Grenzfläche Boden/Luft. Bern, Geographica Bernensia, G37.

EXNER, F.M. (1925): Dynamische Meteorologie. Wien: Julius Springer.

FAIRALL, C. W. (1984): Interpretation of eddy-correlation measurements of particulate deposition and aerosol flux. Atmospheric Environment, 18, 7, S. 1329-1337.

FALK, M. (1997): Turbulente Flüsse von $\mathrm{CO}_{2}, \mathrm{H}_{2} \mathrm{O}$ und fühlbarer Wärme über und in einem Fichtenbestand im Solling. Diplomarbeit, Universität Göttingen. 
FAN, S.; WOFSY, S.C.; BAKWIN, P.S.; JACOB, D.J. (1990): Atmosphere - biosphere exchange of $\mathrm{CO}_{2}$ and $\mathrm{O}_{3}$ in the central Amazon forest. Journal of geophysical Research, 95, S. 1685116864.

FITZGERALD, J.W. (1975): Approximation formulas for the equilibrium size of an aerosol particle as a function of its dry size and composition and the ambient relative humidity. Journal of applied Meteorology, 14, S. 1044-1049.

FOKEN, T. (1990): Turbulenter Energieaustausch zwischen Atmosphäre und Unterlage. Offenbach, Berichte des deutschen Wetterdienstes, Bd. 180.

FOKEN, T.; WICHURA, B. (1996): Tools for quality assessment of surface-based fluxmeasurements. Agricultural and Forest Meteorology, 78, S. 83-105.

FOKEN, T. (1998): Die scheinbar ungeschlossene Energiebilanz am Erdboden - eine Herausforderung an die Experimentelle Meteorologie. Sitzungsberichte der Leibniz-Sozietät, 24, S. 131-150.

FORTAK, H. (1982): Meteorologie. Berlin: Reimer.

Gallagher, M. W.; Beswick, K. M.; Duyzer, J. H.; Westrate, H.; Choularton, T. W.; HuMmelshoI, P. (1997): Measurements of aerosol fluxes to Speulder forest using a micrometeorological technique. Atmospheric Environment, 31, 3, S. 359-373.

GIORGI, F. (1986): A particle dry deposition parameterisation scheme for use in tracer transport models. Journal of geophysical Research, 91, S. 9794-9806.

Gravenhorst, G.; WARAGHAI, A. (1990): Depositionsgeschwindigkeit luftgetragener Partikel für einen Fichtenbestand. In: Berichte des VDI, Bd. 837, S. 119-128.

Gravenhorst, G.; Kreilien, H.; Schnitzler, K.-G.; Ibrom, A.; NÜtZMAnN, E. (2000): Trockene und nasse Deposition von Spurenstoffen aus der Atmosphäre. In: Guderian, R.: Handbuch der Umweltveränderungen und Ökotoxikologie, Berlin, Heidelberg, New York: Springer-Verlag, Bd. 1B: Atmosphäre, S. 147-247.

GRAßL, H. (1999): Wetterwende - Vision: Globaler Klimaschutz. Frankfurt, New York: Campus Verlag, Die Buchreihe der EXPO 2000, Bd. 3.

GROS, D. (1998): Eddykorrelationsmessung an einem Hang. Diplomarbeit, Universität Göttingen.

GRÄDEL, T.E.; CRUTZEN, P.J. (1994): Chemie der Atmosphäre. Heidelberg, Berlin, Oxford: Spektrum Akademischer Verlag. 
Guenther, A.; Baugh, W.; Davis, K.; Hampton, G.; Harley, P.; Klinger, L.; Vierling, L.; Zimmerman, P.; Allwine, E.; Dilts, S.; LAmB, B.; WestberG, H.; BAldocchi, D.; Geron, C.; PIERCE, T. (1996): Isoprene fluxes measured by enclosure, relaxed eddy accumulation, surface layer gradient, mixed layer gradient, and mixed layer mass balance techniques. Journal of geophysical Research, 101, D13, S. 18555-18567.

HAATAJA, J.; VeSAla, T. (Hg.) (1997): SMEAR II. Station for measuring forest ecosystem atmosphere relation. University of Helsinki, Department of Forest Ecology Publications, Bd. 17.

HARTOG, G.D. DEN; NeumanN, H.H. (1984): An empirical study on dry deposition of air pollutants to forests. Project description.

HAUßMANN, T.; LuX, W. (1997): Dauerbeobachtungsflächen zur Umweltkontrolle im Wald Level II: Erste Ergebnisse. Bundesministerium für Ernährung, Landwirtschaft und Forsten.

HERTLEIN, F. (1990): Untersuchungen zur Anwendung der Gradientmethode auf luftgetragene Partikel. Diplomarbeit, Fachhochschule Lübeck, Fachbereich Angewandte Naturwissenschaften.

Heymann, D. (Hg.) (1995): Elfenbisse - feministische Naturwissenschaft. Mössingen Talheim: Talheimer.

Hicks, B.B.; WeSEly, M.L.; DurhaM, J.L.; Brown, M.A. (1982): Some direct measurements of atmospheric sulphur fluxes over a pine plantation. Atmospheric Environment, 12, S. 2899-2903.

HiCKS, B.B.; MCMiLLEN, R.T. (1984): A simulation of the eddy accumulation method for measuring pollutant fluxes. J. Clim. Appl. Meteor., 23, S. 637-643.

HicKS, B.B.; MATT, D.R.; MCMILlen, R.T.; WOMACK, J.D.; WeSEly, M.L.; HART, R.L.; COOK, D.R.; LINDBERG, S.E.; DE PENA, R.G.; THOMSON, D.W. (1989): A field investigation of sulfate fluxes to a deciduous forest. Journal of geophysical Research, 94, S. 13003-13011.

Hofschreuder, P.; RÖMER, F.G.; VAN LEEUWEN, N.F.M.; ARENDS, B.G. (1996): Deposition of aerosol on Speulder forest: accumulation experiments. Atmospheric Environment, 31, S. 351-357.

HÄNEL, G.; THUDIUM, J. (1977): Mean bulk densities of dry atmospheric aerosol particles: a summary of measured data. Pure Appl. Geophys., 115, S. 799 - 803.

HÖFKEN, K. D.; GRAVENHORST, G. (1983): Untersuchung über trockenen Deposition von atmosphärischen Aerosol an Buchen- und Fichtenwald. In: Bericht des Umweltbundesamtes, Berlin: Schmidt-Verlag, Bd. 6/83, Teil II, S. 1-41. 
IBrom, A. (1993): Die Deposition und die Pflanzenauswaschung (Leaching) von Pflanzennährstoffen in einem Fichtenbestand im Solling. Dissertation, Universität Göttingen.

Ibrom, A.; Oltchev, A.; Constantin, J.; Marques, M.; Gravenhorst, G. (1995): Die Stickstoffimmision und -deposition in Wäldern. In: Bericht des Umweltbundesamtes, Göttingen, Bd. 28, S. 20-29.

IBROM, A. (1999): Die biophysikalische Steuerung der Kohlenstoffbilanz in einem Fichtenbestand im Solling. Habilitationsschrift, Universität Göttingen, Institut für Bioklimatologie.

JAENICKE, R.; JUNGE, C. (1967): Studien zur oberen Grenzgröße des natürlichen Aerosols. Beiträge Phys. Atmos., 40, S. 129 - 143.

JAENICKE, R. (1988): Aerosol physics and chemistry. In: Landolt-Börnstein: Meteorology, Berlin, Heidelberg, New York: Springer-Verlag, New Series, Bd. 4, S. 391-456.

JAENICKE, R. (1993): Thropospheric aerosols. In: Hobbs, P.V.: Aerosol-cloud-climate interactions, San Diego: Academic Press, International geophysics series, Bd. 54, S. 1-32.

JONAS, R. (1994): Ablagerung und Bindung von Luftverunreinigungen an Vegetation und anderen atmosphärischen Grenzflächen. KFA Jülich, Bd. 1949.

Junge, C. E. (1963): Air chemistry and radioactivity. New York: Academic Press.

KAIMAL, J. C.; WYNGAARD, J. C.; IZUMI, Y.; COTé, O.R. (1972): Spectral characteristics of surface layer turbulence. Quarterly journal of the Royal meteorological Society, 98, S. 563589.

KAIMAL, J. C.; FinNIGAN, J. J. (1994): Atmospheric boundary layer flows: their structure and management. New York, Oxford: Oxford University Press.

KATEN, P.C.; HuBBE, J.M. (1985): An evaluation of optical particle counter measurements of the dry deposition of atmospheric aerosol particles. Journal of geophysical Research, 90, S. 2145-2160.

Lamaud, E.; Brunet, Y.; Labatut, A.; Lopez, A.; FontAn, J.; Druilhet, A. (1994a): The landes experiment: biosphere-atmosphere exchanges of ozone and aerosol particles above a pine forest. Journal of geophysical Research, 99, S. 16511-16521.

LAmaud, E.; Chapuis, A.; Fontan, J.; SERIE, E. (1994b): Measurements and parameterization of aerosol dry deposition in a semi-arid area. Atmospheric Environment, 28, 15, S. 2461-2471. 
LAUBACH, J. (1991): Bestimmung der vertikalen turbulenten Wärme- und Wasserdampfstromdichten über einem Fichtenwald mit der Methode der Eddy-Korrelation. Diplomarbeit, Universität Göttingen.

LAUBACH, J.; RASCHENDORFER, M.; KREILEIN, H.; GRAVENHORST, G. (1994): Determination of heat and water vapour fluxes above a spruce forest by eddy correlation. Agricultural and Forest Meteorology, 71, S. 373-401.

LEE, X.; BLACK, T.A. (1994): Relating eddy correlation sensible heat flux to horizontal sensor separation in the unstable atmospheric surface layer. Journal of geophysical Research, 99, D9, S. 18.545-18.553.

LENSCHOW, D. H.; RAUPACH, M. R. (1991): The attenuation of fluctuations in scalar concentrations through sampling tubes. Journal of geophysical Research, 96, D8, S. 1525915268 .

Leser, H.; HaAs, H.-D.; Mosimann, T.; PAeSler, R.; Huber-FrÖHlich, J. (1997): Wörterbuch Allgemeine Geographie. München, Braunschweig: Diercke.

Leuning, R.; DenMeAd, O. T.; LANG, A. R. G.; OHTAKI, E. (1982): Effects of heat and water vapor transport on eddy covariance measurement of $\mathrm{CO}_{2}$ fluxes. Boundary-Layer Meteorology, 23, S. 209-222.

LEUNING, R.; LEGG, B.J. (1982): Comments on 'The influence of water vapor fluctuations on turbulent fluxes' by Brook. Boundary-Layer Meteorology, 23, S. 255-258.

LEUNING, R.; MONCRIEFF, J. (1990): Eddy-covariance $\mathrm{CO}_{2}$ flux measurements using openand closed-path $\mathrm{CO}_{2}$-analysers: corrections for analyser water vapour sensitivity and damping of fluctuations in air sampling tubes. Boundary-Layer Meteorology, 53, S. 63-76.

LILJEQUIST, G.H.; CEHAK, K. (1979): Allgemeine Meteorologie. Braunschweig: Vieweg.

LIU, B.Y.H.; PUI, D.Y.H. (1974): A submicron aerosol standard and the primary absolute calibrations of the condensation nucleus counter. J. Coll. Int. Sci., 47, S. 155-171.

LORENZ, R.; MURPHY, C. E. (JR.) (1989): Dry deposition of particles to a pine plantation. Boundary-Layer Meteorology, 46, S. 355-366.

LÖFFLER, J. (1994): Energiebilanz eines Fichtenbestandes: Eddykorrelationsmessungen der turbulenten Wärmeströme mit neuen Flußkorrekturverfahren. Diplomarbeit, Universität Göttingen.

MaitAni, T. (1993): Measurement of $\mathrm{CO}_{2}$ flux in plant communities. J. Agr. Met., 48, 5, S. 551-558. 
MARPLE, V.A.; WiLLEKE, K. (1976): Inertial impactors: theory, design and use. In: Liu, B. Y. H.: Fine particles, aerosol generation, measurement, sampling and analysis, New York: Academic Press, S. 411 - 446.

MARQUES, M.C. (1998): Eintrag von luftgetragenen partikelgebundenen Spurenstoffen in Wälder durch trockene Deposition. Dissertation, Universität Göttingen.

MASSMAN, W.J. (1991): The attenuation of concentration fluctuations in turbulent flow through a tube. Journal of geophysical Research, 96, S. 15,269 - 15,273.

MATZNER, E.; MURACH, D. (1995): Soil changes induced by air pollutant deposition and their implication for forests in central Europe. Water Air Soil Pollut., 85, S. 63-76.

MCMiLLEN, R.T. (1988): An eddy correlation technique with extended applicability to nonsimple terrain. Boundary-Layer Meteorology, 43, S. 231-245.

MeesenburG, H.; Meiwes, J.; Schulze, A.; RAdeMACHER, P. (1997): Bodendauerbeobachtungsflächen auf forstlich genutzten Böden (BDF-F). In: Kleefisch, B.; Kues, J.: Das Bodendauerbeobachtungsprogramm von Niedersachsen: Methodik und Ergebnisse, Hannover, Arb.-H. Boden, Heft 2/1997, S. 77-103.

MEYERS, T.P.; BALDOCCHI, D.D. (1988): A comparison of models for deriving dry deposition fluxes of $\mathrm{O}_{3}$ and $\mathrm{SO}_{2}$ to a forest canopy. Tellus, B 40, S. 270-284.

Michaelis, W.; RePelniK, R.; THeOPOlD, F.; RADEMACHER, P. (1992): Deposition atmosphärischer Spurenstoffe und Stofflüsse im Ökosystem Wald. In: Bauch, J.; Michaelis, W.: Das Forschungsprogramm Waldschäden am Standort "Postturm", Forstamt Farchau / Ratzeburg, GKSS-Forschungszentrum Geesthacht GmbH, GKSS 92/E/100, S. 11-60.

MIE, G. (1908): Beiträge zur Optik trüber Medien, speziell kolloidaler Metallösungen. Annalen der Physik, 25, 3, S. 377-445.

MonCriefF, J. B.; BeVERlAnd, I.J.; NeILl, D.H.; Cropley, F.D. (1998): Controls on trace gas exchange observed by a conditional sampling method. Atmospheric Environment, 32, 19, S. 3265-3274.

MONIN, A.S.; OBUKHOV, A.M. (1954): Basic lows of turbulent mixing in the surface layer of the atmosphere. Trudy Geofiziceskogo Instituta. Akademija nauk SSSR, 24, S. 163-187.

MonTEITH, J.L. (1975): Vegetation and the atmosphere. London, New York, San Francisco: Academic Press, Bd. 1.

MOORE, C.J. (1986): Frequency response corrections for eddy correlation systems. BoundaryLayer Meteorology, 37, S. 17-35. 
MORGENSTERN, K, (2000): Turbulent $\mathrm{CO}_{2-}, \mathrm{H}_{2} \mathrm{O}$ - and energy fluxes above a mediterranean oak and a mountainous spruce forest investigated by eddy-covariance measurements. Dissertation, Universität Göttingen.

Mäkelä, J.M.; Aalto, P.; Jokinen, V.; Pohja, T.; Nissinen, A.; Palmroth, S.; MARKKANEN, T.; SEITSONEN, K.; LiHAVAINEN, H.; KUlMALA, M. (1997): Observations of ultrafine aerosol particle formation and growth in boral forest. Geophysical Research Letters, 24, 10, S. 1219-1222.

NEUMANN, H.H.; HARTOG, G.D. DEN (1985): Eddy correlation measurements of atmospheric fluxes of ozone, sulphur, and particulates during the champaign intercomparison study. Geophysical Research, 90, D1, S. 2097-2110.

NeUMANN, H.H.; HARTOG, G.D. DEN; GUISE-BAGLEY, L.F. (1989): Evaluation of a digitalvalve eddy accumulator using water vapour flux measurements and numerical simulations of its performance. Atmospheric Environment, 23, 6, S. 1305-1313.

NiCHOLlS, S.; SMITH, F.B. (1982): On the definition of the flux of sensible heat. BoundaryLayer Meteorology, 24, S. 121-127.

Panin, G.N.; TetzlafF, G.; RAABe, A.; SchÖNfeldT, H.-J.; NASOnOV, A.E. (1996): Inhomogeneity of the land surface and the parametrization of surface fluxes - a discussion. Wiss. Mitt. Inst. f. Meteorol. Univ. Leipzig \& Inst. f. Troposphärenforschung Leipzig, 4, S. 204-215.

PANOFSKY, H.A.; DUTTON, J.A. (1984): Atmospheric turbulence: models and methods for engineering applications. New York: Wiley.

PERrin, B. (1999): The basics of thermocouples. Circuit Cellar, 12/99, S. 1-5.

PETERS, K.; EIDEN, R. (1992): Modeling the dry deposition velocity of aerosol particles to a spruce forest. Atmospheric Environment, 26A, S. 2555-2564.

PHILIP, J.R. (1963): The damping of a fluctuating concentration by continuous sampling through a tube. Aust. J. Phys., 16, S. 454-463.

PICHLER, H. (1986): Dynamik der Atmosphäre. Mannheim, Wien, Zürich: Bibliographisches Institut.

RANNIK, Ü.; VESALA, T.; KESKINEN, R. (1997): On the damping temperature fluctuations in a circular tube relevant to the eddy-covariance measurement technique. Journal of geophysical Research, 102, S. 12789-12794. 
RASCHENDORFER, M. (1991): Charakterisierung turbulenter atmosphärischer Austauschprozesse in und über einem Waldbestand durch Messungen und Literaturstudium. Diplomarbeit, Universität Göttingen.

REINKING, R.F. (1980): The respective effects of water vapor and temperature on the turbulent fluxes of sensible and latent heat. Boundary-Layer Meteorology, 19, S. 373-385.

RIESE, O. (1995): Turbulenter Austausch in und über einem Fichtenwald: EddyKorrelationsmessungen vertikaler Ströme latenter und fühlbarer Wärme. Diplomarbeit, Universität Göttingen.

RißMANN, J.; TETZLAFF, G. (1994): Application of a spectral correction method for measurements of covariances with fast-response sensors in the atmospheric boundary layer up to a height of $130 \mathrm{~m}$ and testing of the corrections. Boundary-Layer Meteorology, 70, S. 293305.

RoEDEL, W. (1994): Physik unserer Umwelt: Die Atmosphäre. Berlin, Heidelberg, New York: Springer-Verlag, 2. Auflage.

RUIJgROK, W.; TIEBEN, H.; EISINGA, P. (1997): The dry deposition of particles to a forest canopy: a comparison of model and experimental results. Atmospheric Environment, 31, 3, S. $399-415$.

SCHELL, R.; KRISTEN, U. (1992): Trichloressigsäure begünstigt Pilzinfektionen von Fichtennadeln. In: Das Forschungsprogramm Waldschäden am Standort "Postturm", Forstamt Farchau / Ratzeburg, GKSS-Forschungszentrum Geesthacht GmbH, GKSS 92/E/100, S. 353363.

SCHLEGEL, H. (1989): Die Aufnahme von Aerosolen über Fichtennadeln und ihre physiologischen Wirkungen auf die Fichte (Picea abies Karst.). Universität Göttingen, Berichte des Forschungszentrums Waldökosysteme.

SCHMAUSS, A.; WigAND, A. (1929): Die Atmosphäre als Kolloid. Braunschweig: Viehweg \& Sohn.

SCHMID, H.P.; OKE, T.R. (1988): Estimating the source area of a turbulent flux measurement over a patchy surface. Proceedings of Proceedings 8th symphosium on turbulence \& diffusion, San Diego, CA, April 26-29, 1988, Am. Meteorol. Soc.

SCHUEPP, P.H.; LECLERC, M.Y.; MCPHERSON, J.I.; DESJARDIN, R.L. (1990): Footprint prediction of scalar fluxes from analytical solutions of the diffusion equation. BoundaryLayer Meteorology, 50, S. 355-374. 
SCHÜTZ, C. (1996): Eddy-Korrelationsmessungen von $\mathrm{CO}_{2}$-Flüssen im Solling - Aufbau und Test eines Meßsystems. Diplomarbeit, Universität Göttingen.

SEHMEL, G.A.; HodgSON, W.H. (1980): A model for predicting dry deposition of particles and gases to environmental surfaces. AIChE Symp. Ser., 76, S. 218-230.

SHUTTLEWORTH, W.J. (1987): Corrections for the effect of background concentration change and sensor drift in real-time eddy correlation systems. Boundary-Layer Meteorology, 42, S. 167-188.

SLINN, W.G.N. (1982): Predictions for particle deposition to vegetative surfaces. Atmospheric Environment, 16, S. 1785-1794.

SPeER, R.E.; Peterson, K.A.; Ellestad, T.G.; Durham, J.L. (1985): Test of a prototype eddy accumulator for measuring atmospheric vertical fluxes of water vapor and particulate sulfate. Journal of geophysical Research, 90(D1), S. 2119-2122.

SPRANGER, T. (1994): Depositionsmonitoring auf internationaler und nationaler Ebene: Ziele, Eigenschaften und Problembereiche. In: Gravenhorst, G.: Gemeinsames Konzept der langfristigen Erfassung von Stofftransporten zwischen terrestrischen Ökosystemen und der Atmosphäre, Berichte des Forschungszentrums Waldökosysteme, Reihe B, Bd. 41, S. 5-7.

STULL, R.B. (1988): An introduction to boundary layer meteorology. Dordrecht: Kluwer Academic.

StULl, R.B. (1997): Reply. J. Atmosph. Sci., 54, 4, S. 579.

SUYKER, A.E.; VERMA, S.B. (1993): Eddy correlation measurement of $\mathrm{CO}_{2}$ flux using a closed-path sensor: theory and field tests against an open-path sensor. Boundary-Layer Meteorology, 64, S. 391-407.

TWOREK, T. (1996): Feldmessungen von turbulenten Transporten von Wasserdampf und Kohlendioxid über und in einem Fichtenbestand. Diplomarbeit, Universität Göttingen.

UlRICH, B.; MAYER, R.; KHANNA, P.K. (1979): Die Deposition von Luftverunreinigungen und ihre Auswirkungen in Waldökosystemen im Solling. Schriften aus der Forstl. Fak. d. Univ. Göttingen, Reihe B, Bd. 26.

Unland, H.E.; Houser, P.R.; ShutTlewORTh, W.J.; YANG, Z.-L. (1996): Surface flux measurement and modeling at a semi-arid Sonoran Desert site. Agricultural and Forest Meteorology, 82, S. 119-153. 
Vermeulen, A.T.; Wyers, G.P.; RÖMER, F.G.; VAN LEEUWEN, N.F.M.; DrAAIJERS, G.P.J.; ERISMAN, J.W. (1997): Fog deposition on coniferous forest in The Netherlands. Atmospheric Environment, 31, S. 375-386.

Vogt, R. (1995): Theorie, Technik und Analyse der experimentellen Flussbestimmung am Beispiel des Hartheimer Kiefernwaldes - Ein Beitrag zu den Energiebilanzuntersuchungen im REKLIP. Basel, Stratus, Bd. 3.

VONG, R.J.; KOWALSKI, A.S. (1995): Eddy correlation measurements of size-dependent cloud droplet turbulent fluxes to complex terrain. Tellus, 47B, S. 331-352.

WARAGHAI, A.; GRAVENHORST, G. (1989): Dry deposition of atmospheric particles to an old spruce stand. In: Gerorgii, H.-W.: Mechanisms and effects of pollutant-transfer into forests, Dordrecht, Boston, London: Kluwer Academic Publishers, S. 77-86.

WARNECK, P. (2000): Chemistry of the natural atmosphere. San Diego (u.a.): Academic Press, International geophysics series, Bd. 71.

WARNECKE, G. (1997): Meteorologie und Umwelt: Eine Einführung. Berlin, Heidelberg, New York: Springer-Verlag.

WeBB, E.K.; PEARMAN, G.I. (1977): Corrections of $\mathrm{CO}_{2}$ transfer measurements for the effects of water vapour transfer. In: Bilger, R.W.: Second Australasian Conference on Heat and Mass Transfer, University of Sydney, S. 469-476.

WebB, E. K.; PeArman, G. I.; Leuning, R. (1980): Correction of flux measurements for density effects due to heat and water vapour transfer. Quarterly journal of the Royal meteorological Society, 106, S. 85-100.

WEBB, E.K. (1982): On the correction of flux measurements for effects of heat and water vapour transfer. Boundary-Layer Meteorology, 23, S. 251-254.

WEISCHET, W. (1995): Einführung in die allgemeine Klimatologie. Stuttgart: Teubner.

WENT, F.W. (1960): Blue hazes in the atmosphere. Nature, 187, S. 641-643.

Wesely, M.L.; Hicks, B.B.; DANNeVIK, W.P.; Frisella, S.; Husar, R.B. (1977): An eddy correlation measurement of particulate deposition from the atmosphere. Atmospheric Environment, 11, S. 562-563.

WeSEly, M.L.; COOK, D.R.; HART, R.L.; SPEER, R.E. (1985): Measurements and parameterization of particulate sulfur dry deposition over grass. Journal of geophysical Research, 90(D1), S. 2131-2143. 
WiLleKe, K.; BARON, P.A. (Hg.) (1993): Aerosol measurement: principles, techniques, and applications. New York: Van Nostrand-Reinhold.

Wiman, B.L.B.; Agren, G.I. (1985): Aerosol depletion in forests - a model analysis. Atmospheric Environment, 10, S. 335-347.

Wiman, B.L.B.; Unsworth, M.H.; LindBerG, S.E.; BergKWIST, B.; JAEnICKe, R.; HANSSON, H.-C. (1990): Perspectives on aerosol deposition to natural surfaces: interactions between aerosol residence time, removal processes, the biosphere and global environment change. Journal Aerosol Sci., 21, 3, S. 313-338.

WINKLER, P. (2000): Verteilung und Chemie des atmosphärischen Aerosols. In: Guderian, R.: Handbuch der Umweltveränderungen und Ökotoxikologie, Berlin, Heidelberg, New York: Springer-Verlag, Bd. 1B: Atmosphäre, S. 1-39.

Wyers, G.P.; VeltKAMP, A.C. (1996): Dry deposition of 214Pb to conifers. Atmospheric Environment, 31, S. 345-350.

WYERS, G.P.; DUYZER, J.H. (1997): Micrometeorological measurement of the dry deposition flux of sulphate and nitrate aerosols to coniferous forest. Atmospheric Environment, 31, 3, S. 333-343.

XU, X. (2001): Investigations into the tropospheric cycle of COS: atmospheric distribution, air-sea and air-vegetation exchanges. Dissertation, Fachbereich Geowissenschaften / Geographie der Johann Wolfgang Goethe-Universität in Frankfurt. 


\section{Anhang: Untersuchungen von Sonics der Firma Metek im Wind- kanal der ETH Zürich im Rahmen der vorliegenden Arbeit}

\section{Ziel und Rahmen der Windkanaluntersuchungen}

Vom 22.-26.03.1999 fanden im großen Windkanal der Eidgenössischen Technischen Hochschule (ETH) Zürich Untersuchungen an insgesamt elf Ultraschallanemometern (Sonics) der Firmen Campbell, Gill und Metek statt. Ziel der Untersuchungen war es, die von den Sonics im Kanal bestimmten dreidimensionalen Windvektoren mit den tatsächlichen Kanalgeschwindigkeiten zu vergleichen, Abweichungen der Messwerte in Abhängigkeit von Neigung und Azimut der Sonics gegenüber dem Strömungsfeld zu quantifizieren sowie Korrekturfunktionen zu erarbeiten. Die Untersuchungen wurden vorbereitet und durchgeführt durch Dr. Roland Vogt, Institut für Meteorologie, Klimatologie und Fernerkundung der Universität Basel.

Im Rahmen der vorliegenden Arbeit beteiligte sich das Institut für Bioklimatologie der Universität Göttingen (IBK) an den Untersuchungen mit drei Sonics der Firma Metek. Hierbei handelte es sich um die folgenden Geräte:

1. SN 99.03006: klassisches USA1 mit zentraler Vertikalstütze (Mittelstange)

2. SN 94.1229: Hybridgerät USAT 3/1: Sonic mit USA1-Kopf (Mittelstange) und USAT3-Elektronik. Dieses Gerät wurde im Anschluss an die Windkanaluntersuchungen für die Feldmessungen im Rahmen der vorliegenden Arbeit verwendet.

\section{SN 98.12015: USA1 mit Außenbügeln}

Die Beteiligung des IBK an den Untersuchungen wurde ermöglicht durch eine finanzielle Unterstützung der Firma Metek.

\section{Der große Windkanal der ETH Zürich}

Der große Windkanal der ETH Zürich wird vom Institut für Fluiddynamik (IFD) der ETH betrieben und dient sowohl der Forschung als auch der Ausbildung von Studenten des IFD. Er wurde in seiner ursprünglichen Form bereits in den dreißiger Jahren erstellt und seither in vielen Schritten umgebaut und modernisiert. Der Strömungskreislauf des Kanals ist geschlossen, die Querschnittsfläche der eigentlichen Messstrecke beträgt 3,0 x 2,1 m. Zwei Gleichstrommotoren mit jeweils $173 \mathrm{~kW}$ Leistung dienen der Strömungserzeugung, die maximal erreichbare Strömungsgeschwindigkeit beträgt ca. $220 \mathrm{~km} / \mathrm{h}$. Für die Bestimmung von Auftriebs-, Widerstands- und Seitenkräften verfügt der Windkanal über eine eingebaute 6-Komponenten Waage (Abb. 45). 

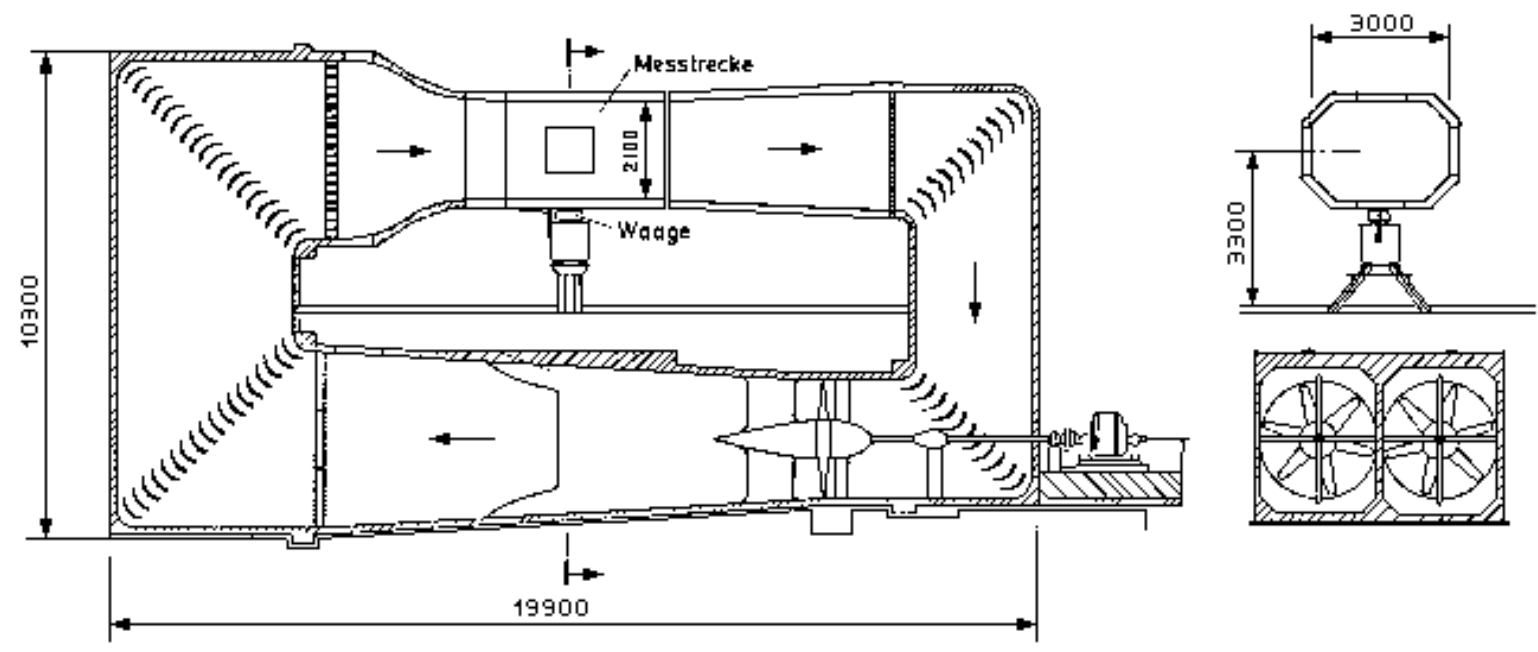

Abb. 45: Seiten- und Frontalansicht des großen Windkanals an der ETH Zürich (alle Längenangaben in Millimeter)

\section{Setup und Kalibration}

Mit jedem Sonic wurden im Windkanal in der Regel vier aufeinanderfolgende, je 55minütige Messreihen aufgenommen, die sich untereinander ausschließlich durch die gesetzte Windgeschwindigkeit im Kanal (2, 4, 6 und $8 \mathrm{~m} / \mathrm{s}$ ) unterschieden. Während je einer Messreihe wurden mit dem zu untersuchenden Gerät jeweils elf verschiedene Vertikalwinkel angefahren $\left(-25,-15,-10,-5,-2,5,0,2,5,5,10,15\right.$ und $25^{\circ}$ Neigung gegen die Horizontale - negative Winkel bedeuten hier, dass das Sonic im Sinne des geräteeigenen Koordinatensystems von unten angeströmt wurde). Für jeden dieser Vertikalwinkel wurde während einer je 5minütigen Messung der dreidimensionale Windvektor für Azimutwinkel zwischen 0 und $360^{\circ}$ bestimmt.

Die Windgeschwindigkeit im Kanal wurde mit einem Propelleranemometer (Auflösung: $0,09 \mathrm{~m} / \mathrm{s}$ ) kontrolliert, Temperatur und Feuchte wurden mit entsprechenden Fühlern kontinuierlich registriert. Zusätzlich wurden Luftdruck und Staudruck in regelmäßigen Abständen manuell abgelesen und notiert. Der Status der Messung ("Messung läuft" und "Messung unterbrochen") wurde - so weit dies an den einzelnen Geräten möglich war - mit Hilfe eines Triggersignals auf einem externen Kanal des jeweiligen Sonics gekennzeichnet.

Die Metek - Sonics wurden kopfüber im Kanal befestigt (Abb. 46). Eine vertikale Ausrichtung der Sonics mit Wasserwaage oder Inklinometer - wie sie bei den Sonics der Firmen Campbell und Gill durchgeführt wurde - konnte für die Metek - Sonics nicht realisiert werden, da die vorhandene Halterung exakt passgerecht gearbeitet war und keine Möglichkeit für Feineinstellungen bot. Die horizontale Ausrichtung der Sonics in die Richtung des Strömungsfeldes des Kanals erfolgte für alle Geräte nach Augenmaß. 
Abb. 46: Kopfüber montierter USA1 Sensorkopf in der Messstrecke des Windkanals. Ebenfalls erkennbar ist der Propelleranemometer (links) und ein Temperaturfühler (Bildmitte). Foto: E. van Gorsel

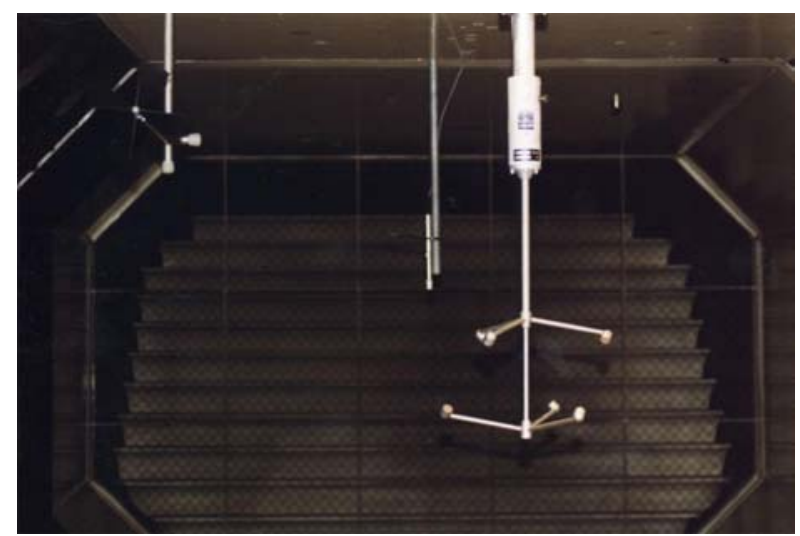

Die Abfrage der Metek - Sonics erfolgte für das Sonic 94.1229 mit dem seit langer Zeit am Institut für Bioklimatologie eingesetztem "Eddy95" - Programm, für die beiden anderen Geräte mit einem neuen, speziell für die Windkanalmessung erstellten Basic - Programm, welches für die Datenübertragung und -abspeicherung eine von der Firma Metek als Zubehör zum USA1 vertriebene Routine ("tcopy.exe") nutzt.

Gegenüber dem ursprünglich geplanten Messablauf ergaben sich für die Metek - Sonics folgende spezielle Änderungen beim Setup:

- Sonic 94.1229: Zusätzlich zu den vier standardmäßigen Messreihen wurden zwei weitere Messreihen gefahren ( 2 und $4 \mathrm{~m} / \mathrm{s}$ ) für die Vertikalwinkel: 34, 30, 20, 12,5, 7,5, 0, -7,5, -12,5, $-20,-30$ und $-34^{\circ}$ gegen die Horizontale.

- Sonic 98.12015: Insgesamt konnten nur zwei Messreihen (2 und $4 \mathrm{~m} / \mathrm{s}$ ) durchgeführt werden.

Sonic 98.12015 und Sonic 99.03006 wurden unmittelbar vor den Messungen in einer thermisch isolierten Box kalibriert. Für das Sonic 94.1229 wurde in der selben Box eine "Nullmessung" durchgeführt. Aus dieser Messung wurde für die x- und y-Komponente des Sonics ein Offset von ca. $2 \mathrm{~cm} / \mathrm{s}$ ermittelt, der in die Auswertung der Daten mit eingeflossen ist.

\section{Auswertung der Daten}

Die Auswertung der Daten erfolgte in folgenden Schritten:

- Zuordnung der gemessenen Werte zu den angefahrenen Vertikal- und Azimutwinkeln über das im externen Kanal aufgezeichnete Triggersignal bzw. - falls kein externer Kanal zur Verfügung stand - über die Maximierung des Korrelationskoeffizienten mit den Daten des 4 m/s - Runs des Sonics 98.12015.

- Für Sonic 98.12015 und Sonic 99.03006: Transformation der Messwerte in das interne USAT3 - Koordinatensystem (Grundlage für eine identische Auswertung aller drei Metek - Sonics). 
- Berechnung von Windgeschwindigkeit, Vertikalkomponente und Windrichtung aus den Messwerten und Vergleich mit den theoretischen Sollwerten.

- Entwicklung einer Korrekturfunktion für das Sonic 94.1229, welche im Rahmen der vorliegenden Arbeit bei der Auswertung von Eddy-Korrelationsmessungen eingesetzt wurde.

\section{Resultate}

\section{Sonic 99.03006}

Die Windgeschwindigkeit (hier: der Betrag des dreidimensionalen Windvektors) wird vom Sonic für die untersuchten Tilt- und Azimutbereiche im allgemeinen gut erfasst. Bei relativ großen Vertikalwinkeln $\left(>15^{\circ}\right)$ kommt es jedoch in Abhängigkeit vom Azimut zu einer Unterschätzung der Windgeschwindigkeit um bis zu $14 \%$. Bei einer nahezu horizontalen Anströmung des Sonics (Vertikalwinkel zwischen 0 bis $+/-10^{\circ}$ ) wird die Windgeschwindigkeit dagegen für einige Azimutbereiche leicht (maximal bis zu 4 bzw. 8 \%) überschätzt. Das Muster der Abweichungen der gemessenen Geschwindigkeiten von der Kanalgeschwindigkeit ist relativ regelmäßig und spiegelt die Sensorgeometrie deutlich wieder. Der relative Betrag der Abweichung ist von der Kanalgeschwindigkeit nur geringfügig abhängig (Abb. 47).

Der Betrag der Vertikalkomponente wird vom Sonic - von einigen Anströmrichtungen mit relativ kleinen Vertikalwinkeln und ganz bestimmten Azimutbereichen abgesehen - praktisch immer unterschätzt. Im Mittel betragen die Differenzen zwischen den Mess- und Sollwerten -8 bis $-12 \%$, weitaus höhere Extremwerte bis zu 100\% - sowohl Unterschätzungen als auch Überschätzungen - findet man dagegen für relativ kleine Vertikalwinkel. Die Unterschätzung der Vertikalkomponente fällt beim Anströmen des Sonics von unten insbesondere bei höheren Kanalgeschwindigkeiten durchschnittlich etwas geringer aus als bei einem Anströmen des Sonics von oben. Der Effekt ist jedoch im Vergleich zum Gesamtbetrag der Unterschätzung sehr gering. Hiervon abgesehen, ist der relative Betrag der Unterschätzung der Vertikalkomponente kaum von der Windgeschwindigkeit abhängig (Abb. 48).

Die Windrichtung wird vom Sonic bei einer nahezu horizontalen Anströmung $\left(0-15^{\circ}\right)$ relativ gut erfasst (max. Abweichung $+/-3^{\circ}$ ). Bei Vertikalwinkeln von größer $+/-15^{\circ}$ erhöht sich die Abweichung vom tatsächlichen Wert auf bis zu $6^{\circ}$ in starker Abhängigkeit vom Azimut. Eine Abhängigkeit des Effektes von der Kanalgeschwindigkeit wurde nicht beobachtet (Abb. 49).

\section{Sonic 94.1229}

Erwartungsgemäß zeigt das Sonic bei der Erfassung der Windgeschwindigkeit eine sehr ähnliche Abhängigkeit von Vertikal- und Azimutwinkeln wie Sonic 99.03006 (und ebenso wie dieses nahezu eine Unabhängigkeit des relativen Fehlers von der Windgeschwindigkeit selbst). Allerdings liegen die gemessenen Werte durchschnittlich um fast $15 \%$ unter den gemessenen Werten des Sonic 99.03006. Das bedeutet, dass die Kanalgeschwindigkeit für 
alle Anströmrichtungen um mindestens $10 \%$, im Mittel um 15 bis $16 \%$ und im Extremfall (Vertikalwinkel von $34^{\circ}$ ) sogar um bis zu $28 \%$ unterschätzt wird. Dieser Unterschied zum Sonic 99.03006 lässt sich nach Auskunft der Firma Metek damit erklären, dass durch die USA1-Elektronik (beim Sonic 99.03006) bereits eine allgemeine, azimut- und tiltunabhängige Korrektur der tatsächlichen Messwerte durchgeführt wird. Da diese Datenmanipulation durch die ältere USAT3-Elektronik (beim USAT 3/1) noch nicht realisiert wird, erhält man hier die tatsächlichen Messergebnisse, die von den realen Windgeschwindigkeiten stärker abweichen als die manipulierten Werte (Abb. 50).

Auch für die Beträge der Vertikalkomponente zeigt sich eine sehr ähnliche Abhängigkeit von Vertikal- und Azimutwinkel wie für das Sonic 99.03006. Wie auch schon bei der Windgeschwindigkeit, fallen erwartungsgemäß bei diesem Sonic aber auch hier die Differenzen zwischen Mess- und Sollwerten deutlich größer aus. Sie betragen im Durchschnitt 23,5 \%. Größere Vertikalwinkel $\left(30,34^{\circ}\right.$ ) führen absolut gesehen zu einer stärkeren Unterschätzung der Vertikalkomponente, relativ gesehen jedoch im allgemeinen nicht (Abb. 51).

Ähnlich wie beim Sonic 99.03006 wird die Windrichtung bei nahezu horizontalen Anströmung gut erfasst, während sich bei größeren Vertikalwinkeln in starker Abhängigkeit vom Azimut Abweichungen von bis $\mathrm{zu} \pm 4^{\circ}$ ergeben. Bei sehr großen Vertikalwinkeln $\left(>25^{\circ}\right)$ wird die Windrichtung teilweise nur noch mit einer Genauigkeit von $6^{\circ}$ gemessen (Abb. 52).

\section{Sonic 98.12015}

Bei kleinen Kanalgeschwindigkeiten $(2 \mathrm{~m} / \mathrm{s}$ ) erfasst das Sonic die Windgeschwindigkeit nur sehr unzureichend. Vor allem in Abhängigkeit vom Azimutwinkel wird die Kanalgeschwindigkeit in den meisten Fällen entweder deutlich über- oder unterschätzt, und zwar jeweils um bis zu $24 \%$. Das Muster der Abweichungen ist weitaus unregelmäßiger als bei den beiden anderen Sonics und lässt sich nicht direkt aus der Sensorgeometrie ableiten. Bei höheren Kanalgeschwindigkeiten $(4 \mathrm{~m} / \mathrm{s})$ wird die Windgeschwindigkeit etwas besser erfasst, die Differenzen zwischen Mess- und Sollwerten verringern sich hier auf -14 bzw. +18 \%. Das Muster der Abweichungen ist aber auch hier noch relativ unregelmäßig (Abb. 53).

Die gemessene Vertikalkomponente ist - absolut gesehen - im Vergleich zu den theoretischen Werten insbesondere bei niedrigen Kanalgeschwindigkeiten häufig zu klein (negativer "Offset" im cm/s bis dm/s Bereich), wobei der Grad der Unterschätzung sowohl von Azimutals auch vom Vertikalwinkel abhängt. Betrachtet man den Betrag der Vertikalkomponente, so wird dieser bei Anströmung von unten folglich in der Regel unterschätzt, bei einer Anströmung von oben jedoch tendenziell überschätzt. Bei größeren Windgeschwindigkeiten $(4 \mathrm{~m} / \mathrm{s})$ wird die Vertikalkomponente etwas besser erfasst, wobei jedoch auch hier noch deutlich unterschiedliche Effekte bei einer Anströmung des Sonics von unten und von oben auffallen. Prozentual sind die Fehler bei einer nahezu horizontalen Anströmung (und damit nur sehr kleinen Vertikalkomponenten) am größten (bis zu +/-100 \%, Abb. 54). 
Die gemessene Windrichtung weicht in starker Abhängigkeit vom Azimutwinkel deutlich von den Sollwerten ab: bei $4 \mathrm{~m} / \mathrm{s}$ um bis zu $+/-10^{\circ}$, bei $2 \mathrm{~m} / \mathrm{s}$ sogar um bis $\mathrm{zu}+/-15^{\circ}$ (Abb. 55 ).

\section{Zusammenfassung}

In einer Untersuchung im Windkanal an der ETH Zürich wiesen die beteiligten Metek Sonics unterschiedliche Messgenauigkeiten auf, wobei mit dem klassischen USA1 - Modell (Sonic 99.03006) nicht zuletzt auf Grund einer internen, im Detail leider nicht dokumentierten Korrektur die besten Resultate erzielen konnten. Die Kombination USA1 - Sensorkopf und USAT3 - Elektronik (Sonic 94.1229) führte zu einer sehr deutlichen, systematischen Unterschätzung sowohl der Windgeschwindigkeit als auch der Vertikalkomponente. Die USA1 - Variante mit Außenbügeln (Sonic 98.12015) lieferte insbesondere für niedrige Windgeschwindigkeiten sehr unbefriedigende Ergebnisse.

Sonic 99.03006 und Sonic 94.1229 zeigen bei der Bestimmung der horizontalen Windgeschwindigkeit, der Windrichtung und der vertikalen Komponente sehr regelmäßige Abhängigkeiten von Azimutwinkel und Vertikalwinkel. Die Kanalgeschwindigkeit selbst hatte dagegen für beide Sonics praktisch keinen Einfluss auf die relativen Differenzen zwischen Mess- und Sollwerten. Deshalb lassen sich für beide Sonics relativ einfach brauchbare Korrekturfunktionen aus den gewonnnen Daten realisieren. Mindestens für das Hybridgerät USAT 3/1 erscheint die Anwendung einer solchen Korrektur für Messungen auch unbedingt erforderlich zu sein, da andererseits unakzeptabel große Abweichungen der Messwerte von den realen Gegebenheiten in Kauf genommen werden müssten (vgl. hierzu auch Abb. 5 dieser Arbeit). Die Erstellung einer Korrekturfunktion für das Sonic 98.12015 ist dagegen deutlich schwieriger, da hier erstens die relativen Beträge der Abweichungen von den Sollwerten u.a. auch von der Windgeschwindigkeit selbst abhängen und zweitens der vom Sonic ermittelte Azimutwinkel teilweise relativ stark von den tatsächlichen Winkeln abweicht. 

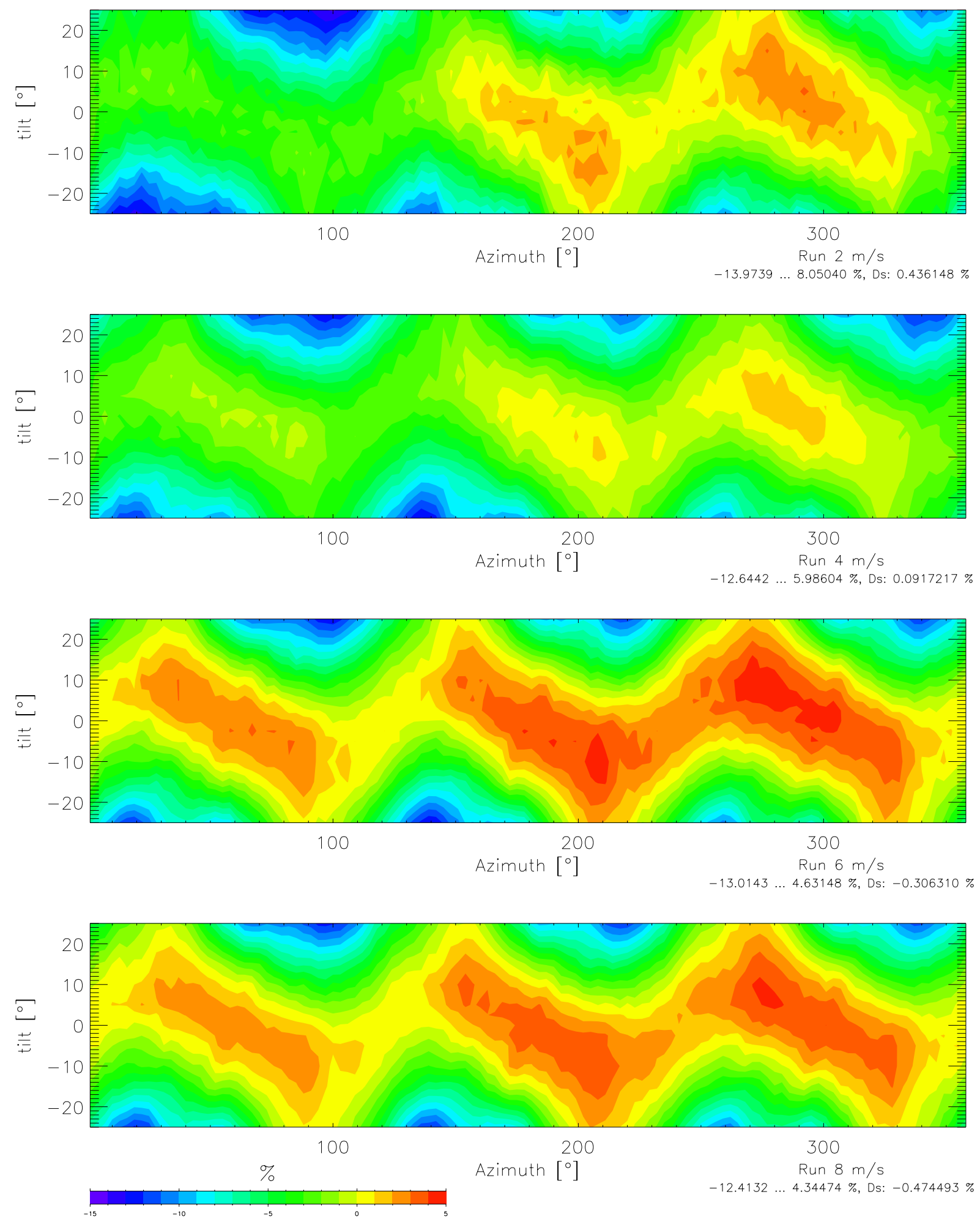

Abb. 47: Abweichung der gemessenen Windgeschwindigkeit (\%) für das Sonic SN 99.03006 als Funktion von Tilt und Azimut für vier verschiedene Anströmgeschwindigkeiten 

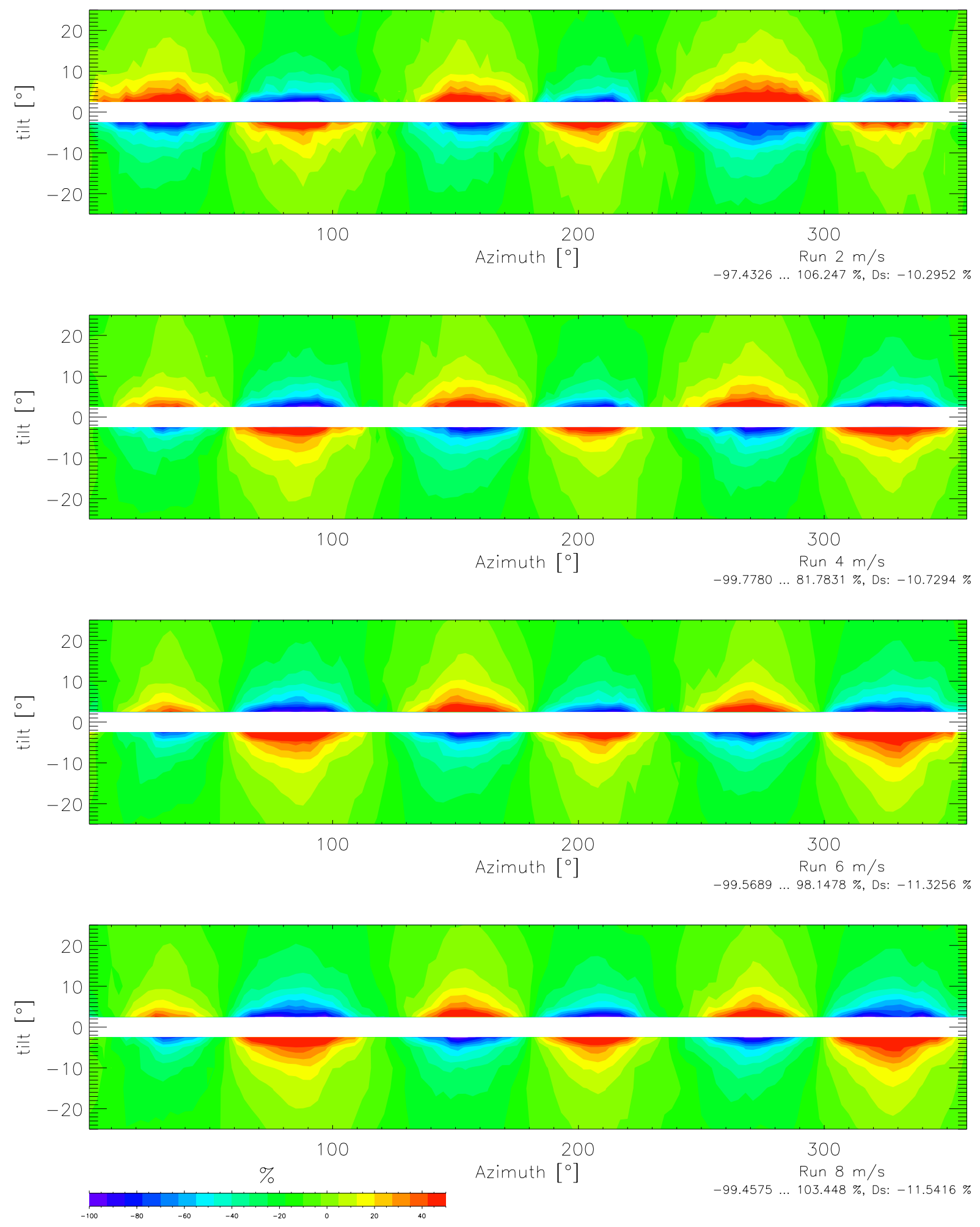

Abb. 48: Abweichung des Betrages der Vertikalkomponente (\%) für das Sonic SN 99.03006 als Funktion von Tilt und Azimut für vier verschiedene Anströmgeschwindigkeiten 

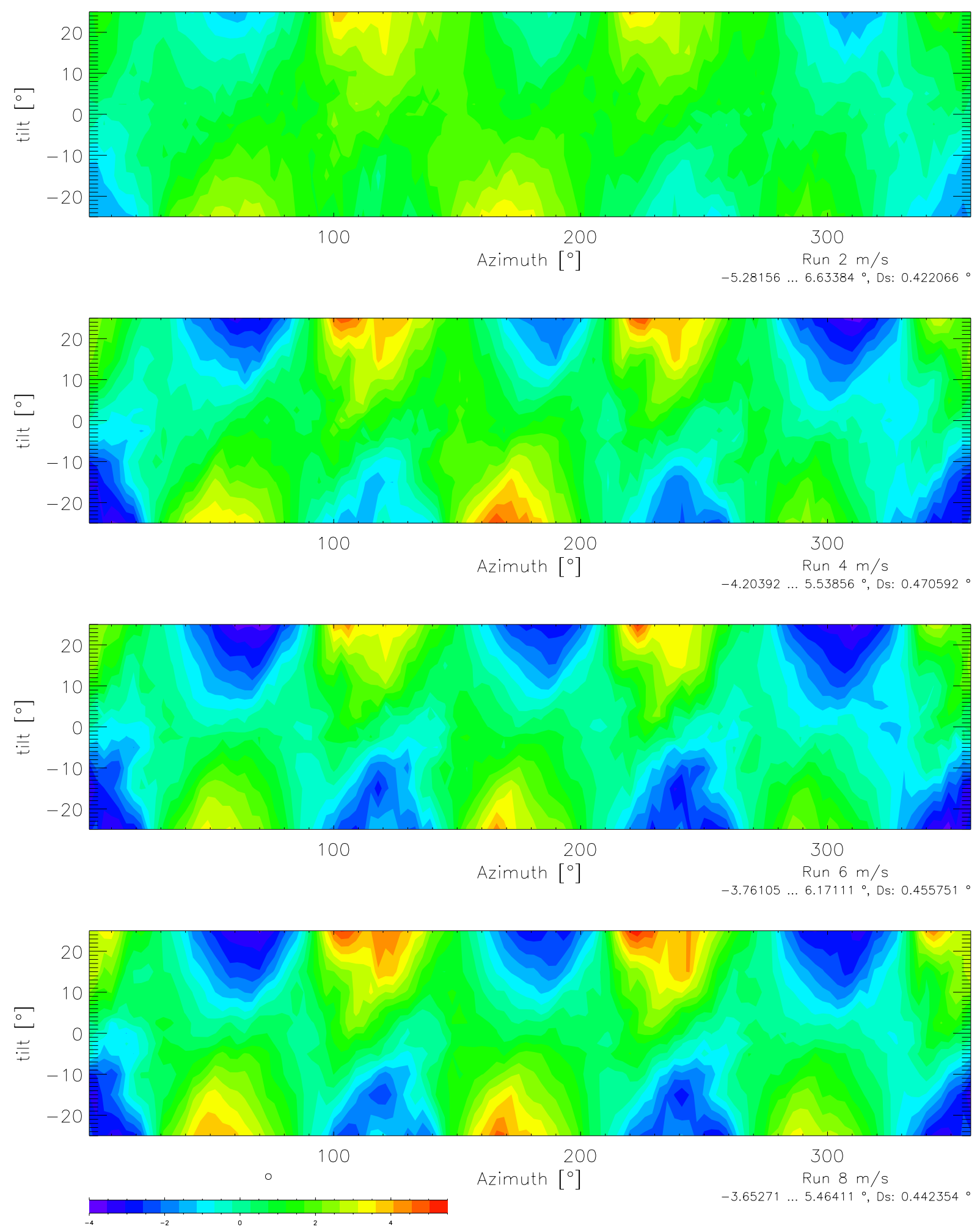

Abb. 49: Abweichung der horizontalen Windrichtung $\left(^{\circ}\right)$ für das Sonic SN 99.03006 als Funktion von Tilt und Azimut für vier verschiedene Anströmgeschwindigkeiten 

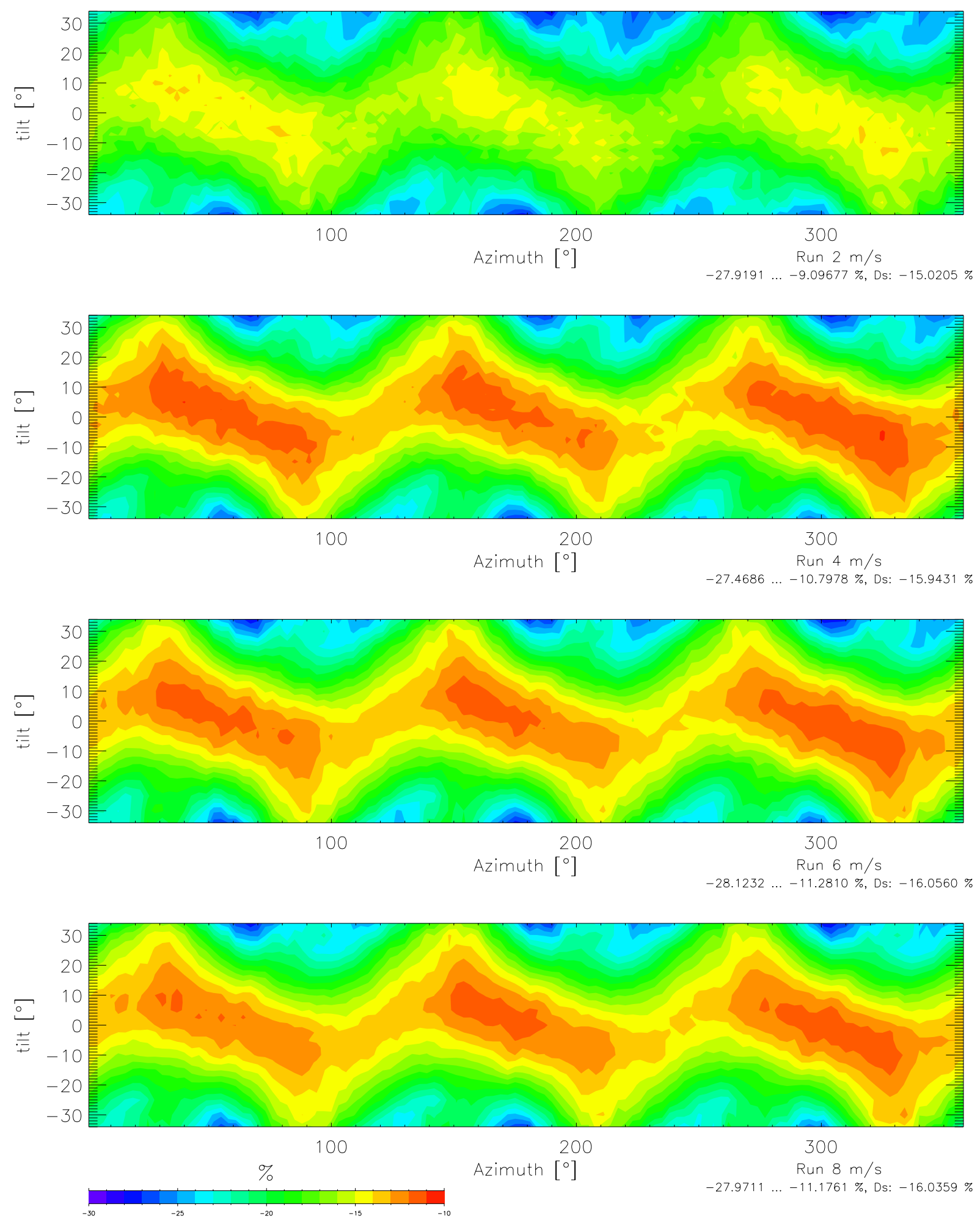

Abb. 50: Abweichung der gemessenen Windgeschwindigkeit (\%) für das Sonic SN 94.1229 als Funktion von Tilt und Azimut für vier verschiedene Anströmgeschwindigkeiten 

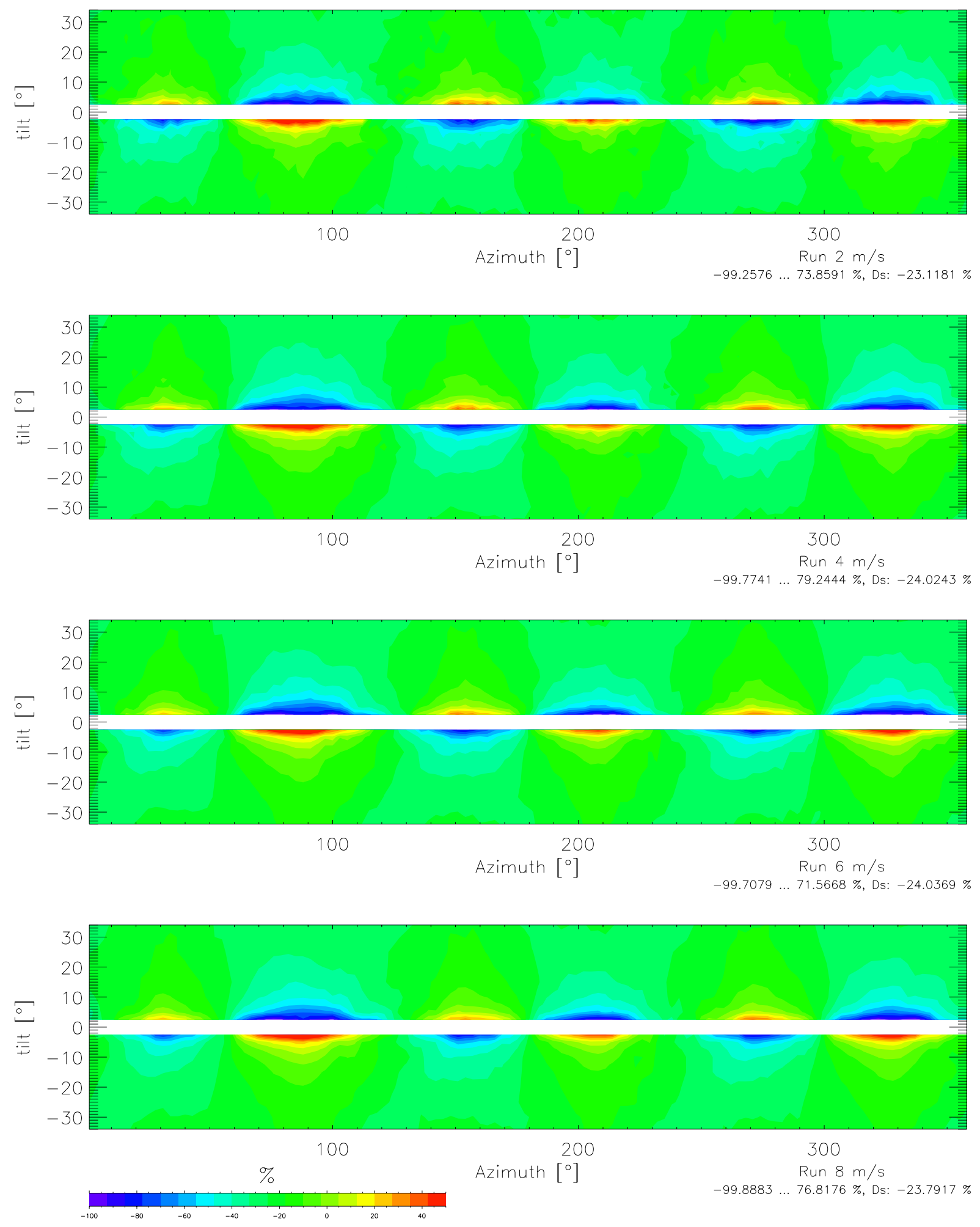

Abb. 51: Abweichung des Betrages der Vertikalkomponente (\%) für das Sonic SN 94.1229 als Funktion von Tilt und Azimut für vier verschiedene Anströmgeschwindigkeiten 

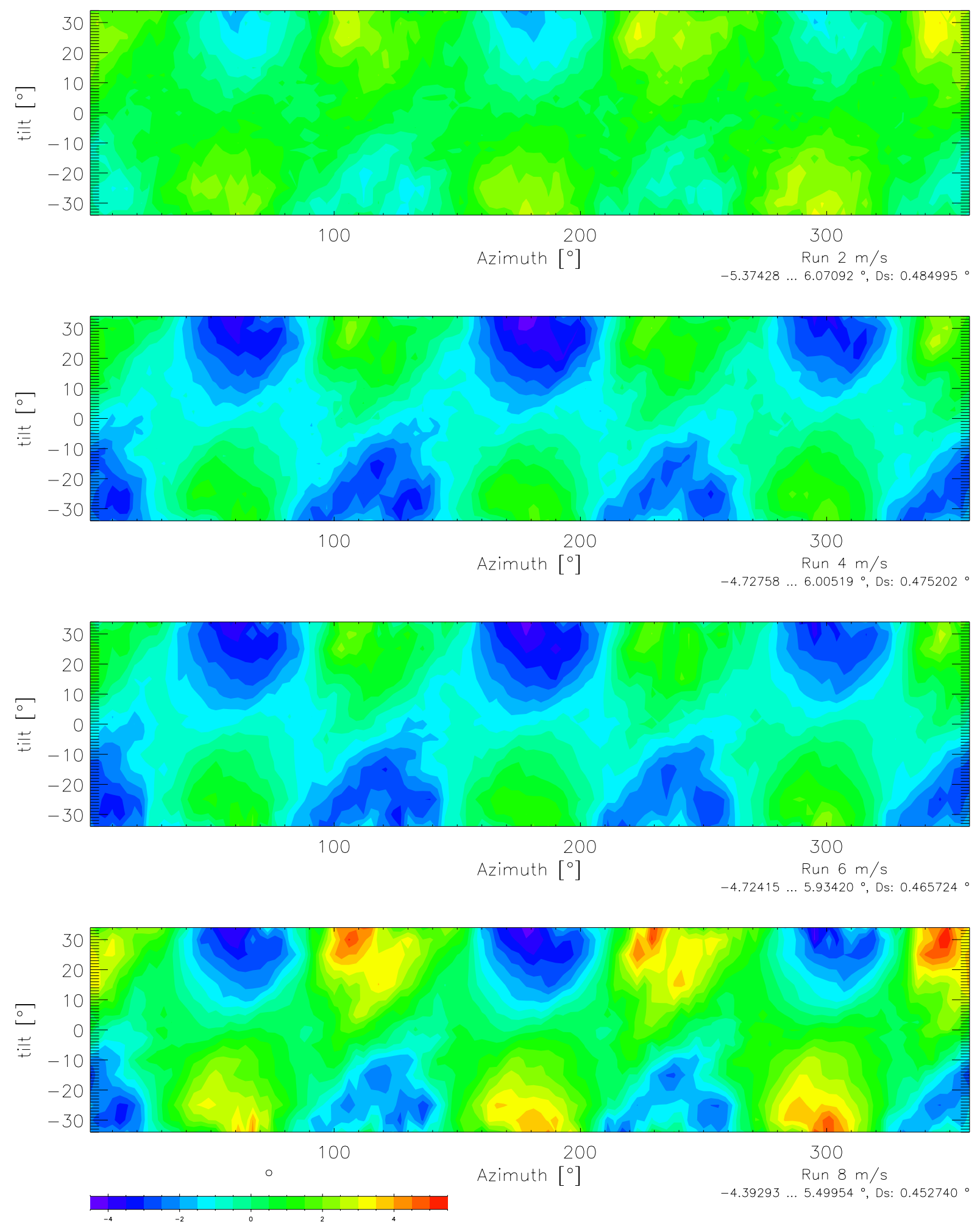

Abb. 52: Abweichung der horizontalen Windrichtung $\left(^{\circ}\right)$ für das Sonic SN 94.1229 als Funktion von Tilt und Azimut für vier verschiedene Anströmgeschwindigkeiten 

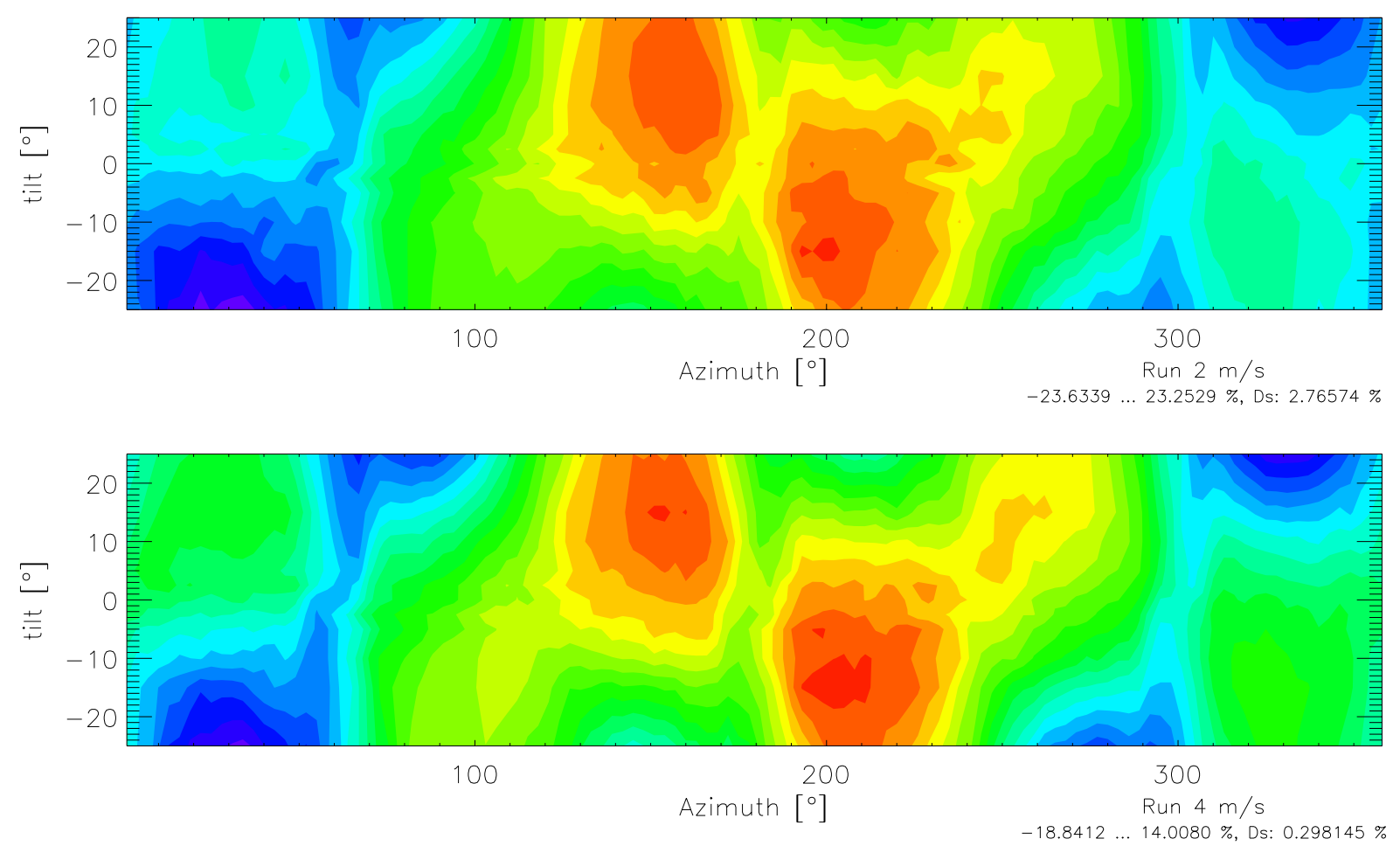

Abb. 53: Abweichung der gemessenen Windgeschwindigkeit (\%) für das Sonic SN 98.12015 als Funktion von Tilt und Azimut für vier verschiedene Anströmgeschwindigkeiten 

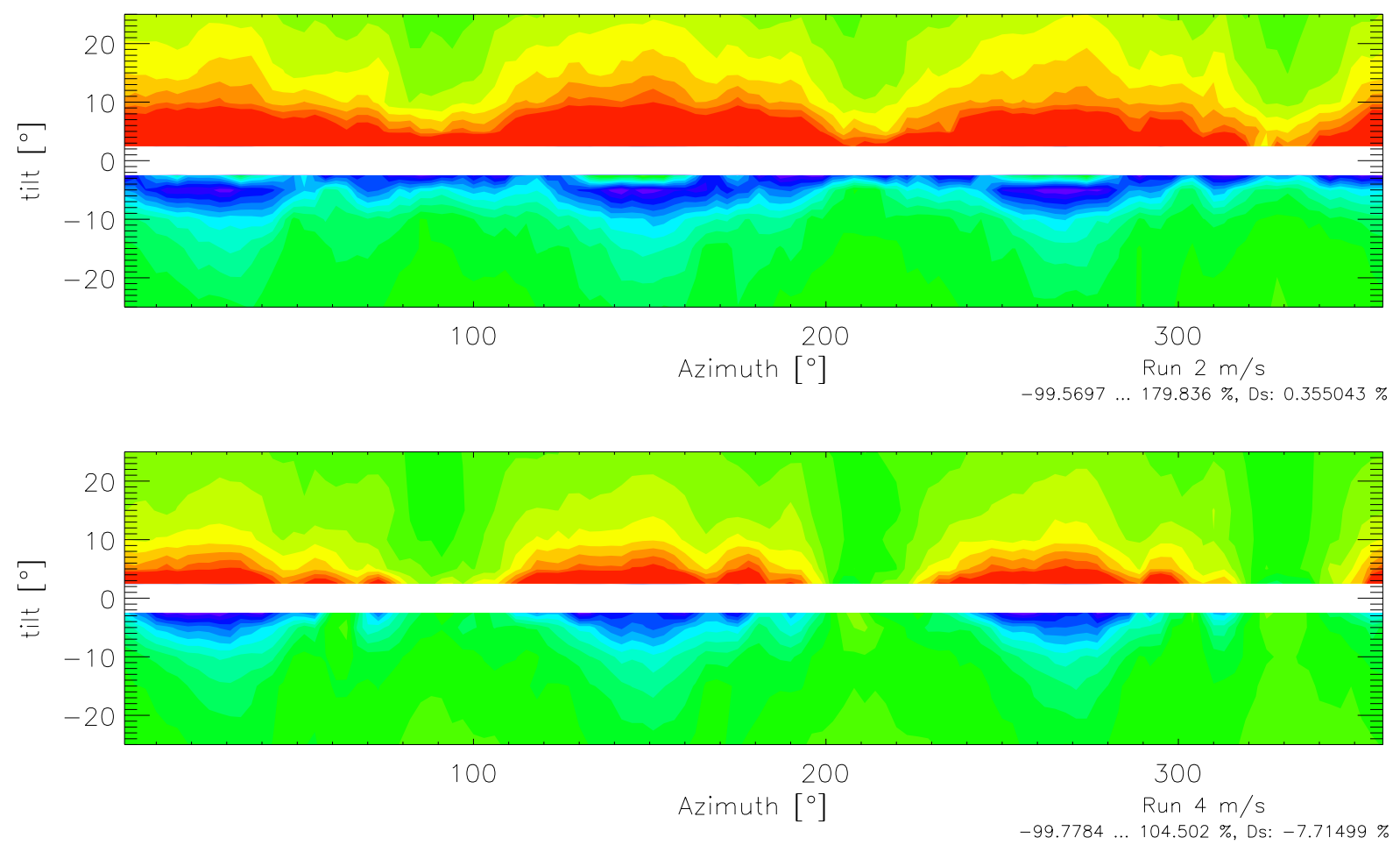

Abb. 54: Abweichung des Betrages der Vertikalkomponente (\%) für das Sonic SN 98.12015 als Funktion von Tilt und Azimut für vier verschiedene Anströmgeschwindigkeiten 

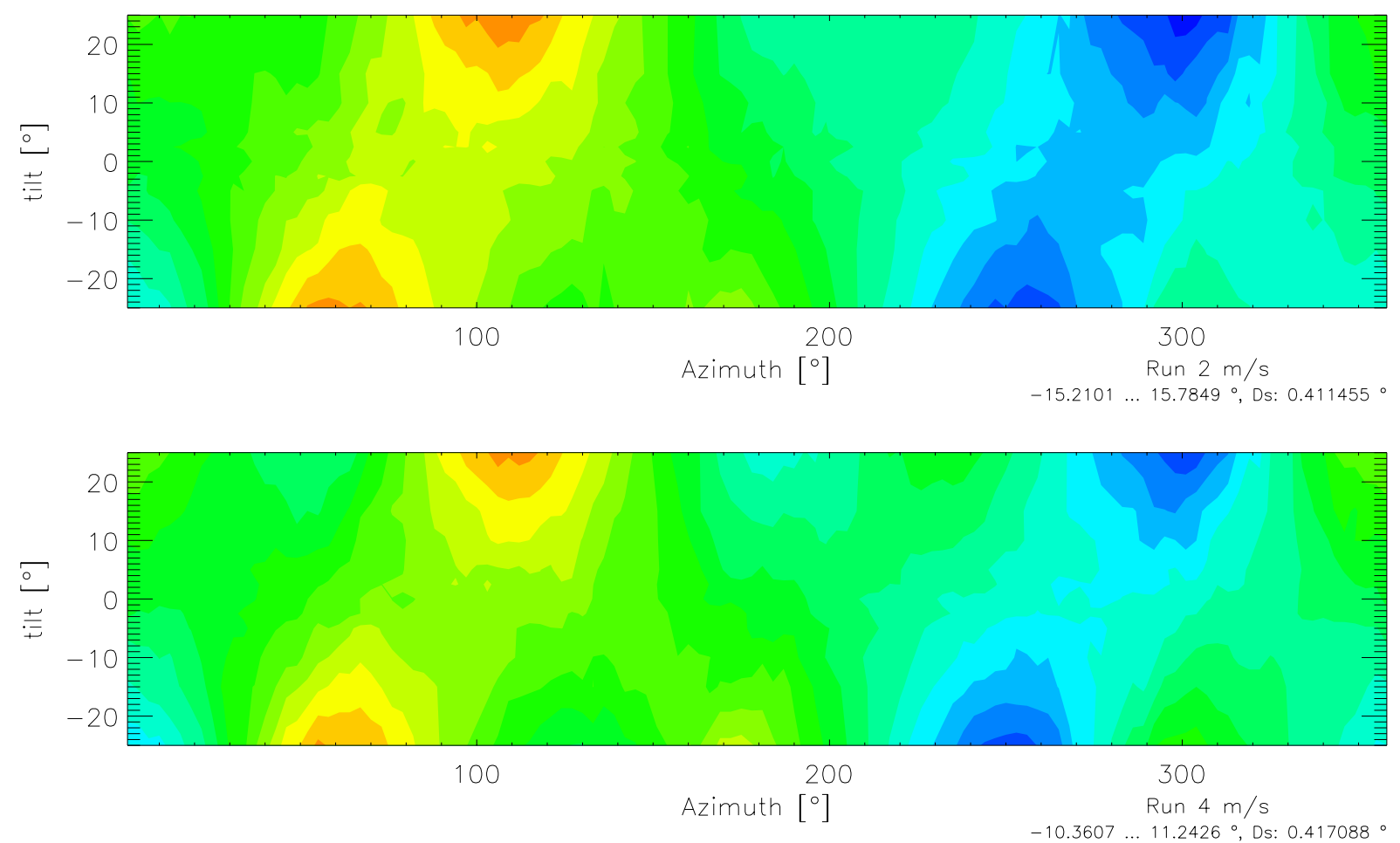

Abb. 55: Abweichung der horizontalen Windrichtung $\left(^{\circ}\right)$ für das Sonic SN 98.12015 als Funktion von Tilt und Azimut für vier verschiedene Anströmgeschwindigkeiten 


\section{Danksagung}

An erster Stelle gilt mein Dank Herrn Prof. Gravenhorst für die interessante Themenstellung, seine kritische und engagierte Begleitung der Arbeit und für wertvolle Diskussionen, die immer wieder neue Anstöße und Fragestellungen aufwarfen.

Mein Dank gilt aber auch allen anderen Mitarbeitern und Mitarbeiterinnen des Instituts für Bioklimatologie, bei denen ich Unterstützung für meine Arbeit gefunden habe. Frau Baumann besorgte mit großem Einsatz die zeit- und nervenraubenden Verwaltungs- und Finanzangelegenheiten. Dietmar Fellert und Kai Freudenstein unterstützen mich tatkräftig bei der Vorbereitung und Durchführung der Messungen im Solling - letzteres auch bei klirrendes Frost und regennasser Witterung. Weitere praktische Hilfe fand ich bei Thomas Grelle, Udo Junghans und Herrn Schäfer. Den Herren Dr. Andreas Ibrom und Dr. Kai Morgenstern danke ich für fruchtbare Diskussionen und Anregungen.

Mein besonderer Dank geht an Dr. Roland Vogt vom Institut für Meteorologie, Klimatologie und Fernerkundung der Universität Basel, der mein Interesse an der Mikrometeorologie geweckt hat, mir wesentlich Fähigkeiten auf diesem Gebiet vermittelt hat und mir im Rahmen der vorliegenden Arbeit die Möglichkeit zur Teilnahme an den Untersuchungen im Windkanal an der ETH Zürich gegeben hat. Finanziell wurde die Beteiligung an diesen Untersuchungen durch eine Unterstützung der Firma Metek ermöglicht - namentlich Herrn Kirzel sei herzlich Dank dafür gesagt.

Bei den Herren Martin Wölz und Thorsten Scharf bedanke ich mich für die Installation eines Linux - Netzwerkes und die sehr gute Realisierung der Eddy2000 Software. Herr Prof. Joachim Saborowski hat mich freundlicherweise bei statistischen Fragestellungen unterstützt. Mit Nicole Walter habe ich einige Zeit am Elektronenmikroskop gearbeitet - besten Dank für die angenehme Zusammenarbeit. Mein besonderer Dank gilt schließlich meinem Vater, meiner Lebenspartnerin Katrin Engelage sowie meinem Freund Matthias Rosenberg für das gründliche Korrekturlesen der Arbeit. 


\section{Lebenslauf}

Name:

Geburtstag:

Staatsangehörigkeit:

schulischer Werdegang:

Berufsausbildung:

Zivildienst:

Studium:

Wissenschaftlicher

Werdegang:

Wissenschaftliche

Interessensgebiete:
Matthias Richard Bleyl

4. 7. 1971, Berlin

deutsch

1978 bis 1991: Oberschule und Gymnasium in Potsdam und Halle/S., Abschluss Abiturzeugnis

1988 bis 1989: Facharbeiter für Getränketechnik, Brauerei Dessau

1991 bis 1992: Rudolf-Steiner-Werkgemeinschaft, Schloss Hamborn, Borchen

1992 bis 1994: Studium der Geoökologie, Universität Potsdam, Abschluss Vordiplom

1995: Gaststudent am Institute of Higher Education, Liverpool 1995 bis 1999: Studium der Geographie und Meteorologie, Universität Basel, Abschluss: Diplom

1999: Diplomarbeit am Institut für Meteorologie, Klimatologie und Fernerkundung an der Universität Basel (Prof. Parlow, Dr. Roland Vogt)

1999 bis 2001: Promotion am Institut für Bioklimatologie der Universität Göttingen (Prof. G. Gravenhorst)

Mikrometeorologie, Eddy - Kovarianzmessungen,

Energiebilanzstudien, wissenschaftliche Programmierung

Göttingen, im Dezember 2001 